\title{
Partial Cauchy Data for General Second Order Elliptic Operators in Two Dimensions
}

\author{
by \\ Oleg Yu. Imanuvilov, Gunther Uhlmann and Masahiro Yamamoto
}

\begin{abstract}
We consider the inverse problem of determining the coefficients of a general second order elliptic operator in two dimensions by measuring the corresponding Cauchy data on an arbitrary open subset of the boundary. We show that one can determine the coefficients of the operator up to natural limitations such as conformal invariance, gauge transformations and diffeomorphism invariance. We use the main result to prove that the curl of the magnetic field and the electric potential are uniquely determined by measuring partial Cauchy data associated to the magnetic Schrödinger equation on an arbitrary open subset of the boundary. We also show that any two of the three coefficients of a second order elliptic operator whose principal part is the Laplacian, are uniquely determined by their partial Cauchy data.
\end{abstract}

2010 Mathematics Subject Classification: 35R30, 35J25.

Keywords: inverse boundary value problem, partial Cauchy data, two-dimensional elliptic operator, uniqueness, inverse anisotropic conductivity problem, magnetic Schrödinger equation, convection equation, complex geometrical optics solution.

\section{$\S 1$. Introduction}

Let $\Omega \subset \mathbb{R}^{2}$ be a bounded domain with smooth boundary $\partial \Omega=\bigcup_{k=1}^{\mathcal{N}} \gamma_{k}$, where $\gamma_{k}$, $1 \leq k \leq \mathcal{N}$, are smooth closed contours, and $\gamma_{\mathcal{N}}$ is the external contour.

Let $\widetilde{\Gamma} \subset \partial \Omega$ be a fixed nonempty relatively open subset of $\partial \Omega$, that is, there exist $\widehat{x} \in \mathbb{R}^{2}$ and $\delta>0$ such that $\emptyset \neq\left\{x \in \mathbb{R}^{2}|| x-\widehat{x} \mid<\delta\right\} \cap \partial \Omega \subset \widetilde{\Gamma}$. Let

Communicated by H. Okamoto. Received August 11, 2011. Revised January 31, 2012.

O. Yu. Imanuvilov: Department of Mathematics, Colorado State University,

101 Weber Building, Fort Collins, CO 80523, USA;

e-mail: oleg@math.colostate.edu

G. Uhlmann: Department of Mathematics, University of California, Irvine,

Irvine, CA 92697, USA, and Department of Mathematics, University of Washington,

Seattle, WA 98195, USA;

e-mail: gunther@math.washington.edu

M. Yamamoto: Department of Mathematical Sciences, The University of Tokyo,

Komaba, Meguro, Tokyo 153, Japan;

e-mail: myama@ms.u-tokyo.ac.jp

(C) 2012 Research Institute for Mathematical Sciences, Kyoto University. All rights reserved. 
$\Gamma_{0}=\partial \Omega \backslash \overline{\widetilde{\Gamma}}$. Let $\nu=\left(\nu_{1}, \nu_{2}\right)$ be the unit outward normal vector to $\partial \Omega$ and let $\frac{\partial u}{\partial \nu}=\nabla u \cdot \nu$.

Henceforth we set $i=\sqrt{-1}, x_{1}, x_{2} \in \mathbb{R}, z=x_{1}+i x_{2}, \bar{z}$ denotes the complex conjugate of $z \in \mathbb{C}$, and we identify $x=\left(x_{1}, x_{2}\right) \in \mathbb{R}^{2}$ with $z=x_{1}+i x_{2} \in \mathbb{C}$. We also denote $\frac{\partial}{\partial \bar{z}}=\frac{1}{2}\left(\frac{\partial}{\partial x_{1}}+i \frac{\partial}{\partial x_{2}}\right)$ and $\frac{\partial}{\partial z}=\frac{1}{2}\left(\frac{\partial}{\partial x_{1}}-i \frac{\partial}{\partial x_{2}}\right)$.

Let $u \in H^{1}(\Omega)$ be a solution to the boundary value problem

$$
L(x, D) u=\Delta_{\mathbf{g}} u+2 A \frac{\partial u}{\partial z}+2 B \frac{\partial u}{\partial \bar{z}}+q u=0,\left.\quad u\right|_{\Gamma_{0}}=0,\left.\quad u\right|_{\widetilde{\Gamma}}=f .
$$

Here $\Delta_{\mathrm{g}}$ denotes the Laplace-Beltrami operator associated to the Riemannian metric $\mathbf{g}=\left\{g_{j k}\right\}$. We assume that $\mathbf{g}$ is a positive definite symmetric matrix in $\Omega$ and

$$
\Delta_{\mathbf{g}}=\frac{1}{\sqrt{\operatorname{det} \mathbf{g}}} \sum_{j, k=1}^{2} \frac{\partial}{\partial x_{k}}\left(\sqrt{\operatorname{det} \mathbf{g}} g^{j k} \frac{\partial}{\partial x_{j}}\right)
$$

where $\left\{g^{j k}\right\}$ denotes the inverse of $\mathbf{g}=\left\{g_{j k}\right\}$. From now on we assume that $\mathbf{g}, \mathbf{g}_{j} \in C^{7+\alpha}(\bar{\Omega})$ and that $(A, B, q),\left(A_{j}, B_{j}, q_{j}\right) \in C^{5+\alpha}(\bar{\Omega}) \times C^{5+\alpha}(\bar{\Omega}) \times C^{4+\alpha}(\bar{\Omega})$, $j=1,2$, for some $\alpha \in(0,1)$ are complex-valued functions. Henceforth $\alpha$ denotes a constant such that $0<\alpha<1$.

We set

$$
L_{j}(x, D)=\Delta_{\mathbf{g}_{j}}+2 A_{j} \frac{\partial}{\partial z}+2 B_{j} \frac{\partial}{\partial \bar{z}}+q_{j}, \quad j=1,2 .
$$

We define the partial Cauchy data by

$$
\begin{array}{r}
\mathcal{C}_{\mathbf{g}, A, B, q}=\left\{\left(\left.u\right|_{\widetilde{\Gamma}},\left.\frac{\partial u}{\partial \nu_{\mathbf{g}}}\right|_{\widetilde{\Gamma}}\right) \mid\left(\Delta_{\mathbf{g}}+2 A \frac{\partial}{\partial z}+2 B \frac{\partial}{\partial \bar{z}}+q\right) u=0 \text { in } \Omega,\right. \\
\left.u \in H^{1}(\Omega),\left.u\right|_{\Gamma_{0}}=0\right\},
\end{array}
$$

where $\frac{\partial}{\partial \nu_{\mathbf{g}}}=\sqrt{\operatorname{det} \mathbf{g}} \sum_{j, k=1}^{2} g^{j k} \nu_{k} \frac{\partial}{\partial x_{j}}$. Henceforth Id denotes the identity mapping from $\mathbb{R}^{2}$ to $\mathbb{R}^{2}$.

The goal of this paper is to determine the coefficients of the operator $L$ from the partial Cauchy data. In the general case this is impossible. The Cauchy data present invariance of the following three types.

(i) The partial Cauchy data for the operators $e^{-\eta} L(x, D) e^{\eta}$ and $L(x, D)$ are the same provided that $\eta \in C^{6+\alpha}(\bar{\Omega})$ is a complex-valued function and $\left.\eta\right|_{\widetilde{\Gamma}}=$ $\left.\frac{\partial \eta}{\partial \nu}\right|_{\widetilde{\Gamma}}=0$.

(ii) Let $\beta \in C^{7+\alpha}(\bar{\Omega})$ be a positive function on $\bar{\Omega}$. The partial Cauchy data for the operators $L(x, D)$ and $\frac{1}{\beta} L(x, D)=\Delta_{\beta \mathbf{g}}+\frac{1}{\beta}\left(2 A \frac{\partial}{\partial z}+2 B \frac{\partial}{\partial \bar{z}}+q\right)$ are exactly the same. 
(iii) Let $F=\left(F_{1}, F_{2}\right) \in C^{8+\alpha}(\bar{\Omega}), F: \bar{\Omega} \rightarrow \bar{\Omega}$ be a diffeomorphism such that $\left.F\right|_{\widetilde{\Gamma}}=$ Id. For any metric $\mathbf{g}$ and complex-valued functions $A, B, q$, we introduce a metric $F^{*} \mathrm{~g}$ and functions $A_{F}, B_{F}, q_{F}$ by

$$
\begin{aligned}
F^{*} \mathbf{g} & =\left((D F) \circ \mathbf{g} \circ(D F)^{T}\right) \circ F^{-1}, \\
A_{F} & =\left|\operatorname{det} D F^{-1}\right|\left\{(A+B)\left(\frac{\partial F_{1}}{\partial x_{1}}-i \frac{\partial F_{2}}{\partial x_{1}}\right)+i(B-A)\left(\frac{\partial F_{1}}{\partial x_{2}}-i \frac{\partial F_{2}}{\partial x_{2}}\right)\right\} \circ F^{-1}, \\
B_{F} & =\left|\operatorname{det} D F^{-1}\right|\left\{(A+B)\left(\frac{\partial F_{1}}{\partial x_{1}}+i \frac{\partial F_{2}}{\partial x_{1}}\right)+i(B-A)\left(\frac{\partial F_{1}}{\partial x_{2}}+i \frac{\partial F_{2}}{\partial x_{2}}\right)\right\} \circ F^{-1}, \\
q_{F} & =\left|\operatorname{det} D F^{-1}\right|\left(q \circ F^{-1}\right),
\end{aligned}
$$

where $D F$ denotes the Jacobian matrix of $F,(D F)^{T}$ its transpose, and o denotes matrix multiplication. Then the operator

$$
K(x, D)=\Delta_{F^{*} \mathbf{g}}+2 A_{F} \frac{\partial}{\partial z}+2 B_{F} \frac{\partial}{\partial \bar{z}}+q_{F}
$$

and the operator $L(x, D)$ have the same partial Cauchy data.

We show the converse and state our main result below.

Assume that for some $\alpha \in(0,1)$ and $\alpha^{\prime}>0$,

$$
g_{j k} \in C^{7+\alpha}(\bar{\Omega}), \quad g_{j k}=g_{k j} \quad \forall k, j \in\{1,2\}, \quad \sum_{j, k=1}^{2} g_{j k} \xi_{k} \xi_{j} \geq \alpha^{\prime}|\xi|^{2}, \quad \forall \xi \in \mathbb{R}^{2} .
$$

Consider the following set of functions:

$$
\eta \in C^{6+\alpha}(\bar{\Omega}),\left.\quad \frac{\partial \eta}{\partial \nu}\right|_{\widetilde{\Gamma}}=0,\left.\quad \eta\right|_{\widetilde{\Gamma}}=0 .
$$

We have

Theorem 1.1. Suppose that for some $\alpha \in(0,1)$, there exists a positive function $\widetilde{\beta} \in C^{7+\alpha}(\bar{\Omega})$ such that $\left.\left(\mathbf{g}_{1}-\widetilde{\beta} \mathbf{g}_{2}\right)\right|_{\widetilde{\Gamma}}=\left.\frac{\partial\left(\mathbf{g}_{1}-\widetilde{\beta} \mathbf{g}_{2}\right)}{\partial \nu}\right|_{\widetilde{\Gamma}}=0$. Then $\mathcal{C}_{\mathbf{g}_{1}, A_{1}, B_{1}, q_{1}}=$ $\mathcal{C}_{\mathbf{g}_{2}, A_{2}, B_{2}, q_{2}}$ if and only if there exist a diffeomorphism $F \in C^{8+\alpha}(\bar{\Omega}), F: \bar{\Omega} \rightarrow \bar{\Omega}$ satisfying $\left.F\right|_{\widetilde{\Gamma}}=\mathrm{Id}$, a positive function $\beta \in C^{7+\alpha}(\bar{\Omega})$ and a complex-valued function $\eta$ satisfying (1.5) such that

$$
L_{2}(x, D)=e^{-\eta} K(x, D) e^{\eta},
$$

where

$$
K(x, D)=\Delta_{\beta F^{*} \mathbf{g}_{1}}+\frac{2}{\beta} A_{1, F} \frac{\partial}{\partial z}+\frac{2}{\beta} B_{1, F} \frac{\partial}{\partial \bar{z}}+\frac{1}{\beta} q_{1, F}
$$

and the functions $F^{*} \mathbf{g}_{1}, A_{1, F}, B_{1, F}, q_{1, F}$ are defined for $\mathbf{g}_{1}, A_{1}, B_{1}, q_{1}$ by (1.4).

Next we discuss the anisotropic conductivity problem. In this case the conductivity depends on direction and is represented by a positive definite symmetric 
matrix $\sigma^{-1}=\left\{\sigma^{j k}\right\}$. The conductivity equation with voltage potential $f$ on $\partial \Omega$ is given by

$$
\sum_{j, k=1}^{2} \frac{\partial}{\partial x_{j}}\left(\sigma^{j k} \frac{\partial u}{\partial x_{k}}\right)=0 \quad \text { in } \Omega,\left.\quad u\right|_{\partial \Omega}=f .
$$

We define the partial Cauchy data by

$$
\begin{array}{r}
\mathcal{V}_{\sigma}=\left\{\left(\left.f\right|_{\widetilde{\Gamma}},\left.\sum_{j, k=1}^{2} \sigma^{j k} \nu_{j} \frac{\partial u}{\partial x_{k}}\right|_{\widetilde{\Gamma}}\right) \mid \sum_{j, k=1}^{2} \frac{\partial}{\partial x_{j}}\left(\sigma^{j k} \frac{\partial u}{\partial x_{k}}\right)=0 \text { in } \Omega\right. \\
\left.u \in H^{1}(\Omega),\left.u\right|_{\partial \Omega}=f, \operatorname{supp} f \subset \widetilde{\Gamma}\right\} .
\end{array}
$$

It is known (see [3]) that even in the case of $\widetilde{\Gamma}=\partial \Omega$, the full Cauchy data $\mathcal{V}_{\sigma}$ does not determine $\sigma$ uniquely in the anisotropic case. Let $F: \bar{\Omega} \rightarrow \bar{\Omega}$ be a diffeomorphism such that $F(x)=x$ for $x \in \widetilde{\Gamma}$. Then

$$
\mathcal{V}_{\left|\operatorname{det} D F^{-1}\right| F^{*} \sigma}=\mathcal{V}_{\sigma}
$$

In the case of full Cauchy data (i.e., $\widetilde{\Gamma}=\partial \Omega$ ) the question whether one can determine the conductivity up to the above limitation, has been solved in two dimensions for $C^{2}$ conductivities in [21], Lipschitz conductivities in [27] and merely $L^{\infty}$ conductivities in [2], [3]. The method of proof in all these papers is reduction to the isotropic case using isothermal coordinates [1]. See also [20]. We have

Theorem 1.2. Let $\sigma_{1}, \sigma_{2} \in C^{7+\alpha}(\bar{\Omega})$ with some $\alpha \in(0,1)$ be positive definite symmetric matrices on $\bar{\Omega}$. If $\mathcal{V}_{\sigma_{1}}=\mathcal{V}_{\sigma_{2}}$, then there exists a diffeomorphism $F$ : $\bar{\Omega} \rightarrow \bar{\Omega}$ satisfying $\left.F\right|_{\widetilde{\Gamma}}=\operatorname{Id}$ and $F \in C^{8+\alpha}(\bar{\Omega})$ such that

$$
\left|\operatorname{det} D F^{-1}\right| F^{*} \sigma_{1}=\sigma_{2} \text {. }
$$

For the isotropic case, the corresponding result is proved in [15].

Next we consider the case where $\mathbf{g}=I$, the $2 \times 2$ identity matrix. We remark that general second order elliptic operators can be reduced to this form by using isothermal coordinates (see, e.g., [1]). In this case, our first result is the following:

Theorem 1.3. Let $A_{1}, A_{2}, B_{1}, B_{2} \in C^{5+\alpha}(\bar{\Omega})$ and $q_{1}, q_{2} \in C^{4+\alpha}(\bar{\Omega})$ with some $\alpha \in(0,1)$. Assume that $\mathcal{C}_{I, A_{1}, B_{1}, q_{1}}=\mathcal{C}_{I, A_{2}, B_{2}, q_{2}}$. Then

$$
\begin{aligned}
& A_{1}=A_{2}, \quad B_{1}=B_{2} \quad \text { on } \widetilde{\Gamma}, \\
& -2 \frac{\partial}{\partial \bar{z}}\left(B_{1}-B_{2}\right)-\left(A_{1}-A_{2}\right) B_{1}-\left(B_{1}-B_{2}\right) A_{2}+\left(q_{1}-q_{2}\right)=0 \quad \text { in } \Omega \text {. }
\end{aligned}
$$


Remark. In the case where $A_{1}=A_{2}=0$ and $B_{1}=B_{2}=0$ in $\Omega$, Theorem 1.3 yields $q_{1}=q_{2}$, which is the main result in [15]. The latter result was extended to Riemann surfaces in [13]. The case of full data in two dimensions was settled in [7]. This case is closely related to the inverse conductivity problem or Calderón's problem (see [9]).

For global uniqueness results in the two-dimensional case for the conductivity equation with full data measurements under different regularity assumptions, see $[2],[6],[16]$ and [21].

Theorem 1.3 yields

Corollary 1.1. Let $A_{1}, A_{2}, B_{1}, B_{2} \in C^{5+\alpha}(\bar{\Omega})$ and $q_{1}, q_{2} \in C^{4+\alpha}(\bar{\Omega})$ with some $\alpha \in(0,1)$. The relation $\mathcal{C}_{I, A_{1}, B_{1}, q_{1}}=\mathcal{C}_{I, A_{2}, B_{2}, q_{2}}$ holds true if and only if there exists a function $\eta \in C^{6+\alpha}(\bar{\Omega})$ with $\left.\eta\right|_{\widetilde{\Gamma}}=\left.\frac{\partial \eta}{\partial \nu}\right|_{\widetilde{\Gamma}}=0$ such that

$$
L_{1}(x, D)=e^{-\eta} L_{2}(x, D) e^{\eta} .
$$

Proof. We only prove the necessity since the sufficiency is easy to check. By (1.8) and (1.9), we have $\frac{\partial}{\partial z}\left(A_{1}-A_{2}\right)=\frac{\partial}{\partial \bar{z}}\left(B_{1}-B_{2}\right)$. This equality is equivalent to

$$
\frac{\partial(\widehat{A}-\widehat{B})}{\partial x_{1}}=i \frac{\partial(\widehat{B}+\widehat{A})}{\partial x_{2}} \quad \text { where } \quad(\widehat{A}, \widehat{B})=\left(A_{1}-A_{2}, B_{1}-B_{2}\right) .
$$

Applying Lemmata 1.1 and 1.2 (pp. 460-462) of [28], we deduce that there exists a function $\widetilde{\eta}$ with domain $\Omega^{0}$ which satisfies

$$
\begin{aligned}
& \widetilde{\eta}=\eta_{0}+h, \nabla \widetilde{\eta} \in C^{5+\alpha}(\bar{\Omega}), \quad \Delta h=0 \quad \text { in } \Omega^{0} \\
& {\left.[h]\right|_{\Sigma_{k}} \text { are constants, }\left.\left[\frac{\partial h}{\partial \nu_{k}}\right]\right|_{\Sigma_{k}}=\left.\frac{\partial h}{\partial \nu}\right|_{\gamma_{\mathcal{N}}}=0 \quad \forall k \in\{1, \ldots, \mathcal{N}\},}
\end{aligned}
$$

and

$$
(i(\widehat{B}+\widehat{A}), \widehat{A}-\widehat{B})=\nabla \widetilde{\eta}
$$

Here $\Omega^{0}=\Omega \backslash \Sigma$ is simply connected where $\Sigma=\bigcup_{k=1}^{\mathcal{N}-1} \Sigma_{k}, \Sigma_{j} \cap \Sigma_{k}=\emptyset$ for $j \neq k$, and $\Sigma_{k}$ are smooth curves which do not self-intersect and are orthogonal to $\partial \Omega$ at the intersection points. In other words, a simply connected domain $\Omega^{0}$ is produced by a finite number of smooth cuts. We choose a normal vector $\nu_{k}=\nu_{k}(x)$, $1 \leq k \leq \mathcal{N}-1$, to $\Sigma_{k}$ at $x$ contained in the interior $\Sigma_{k}^{0}$ of the closed curve $\Sigma_{k}$. Then, for $x \in \Sigma_{k}^{0}$, we set $[h](x)=\lim _{y \rightarrow x,\left(\overrightarrow{x y}, \nu_{k}\right)>0} h(y)-\lim _{y \rightarrow x,\left(\overrightarrow{x y}, \nu_{k}\right)<0} h(y)$ where $(\cdot, \cdot)$ denotes the scalar product in $\mathbb{R}^{2}$. Setting $2 \eta=-i \widetilde{\eta}$, we have

$$
(\widehat{B}+\widehat{A}, i(\widehat{B}-\widehat{A}))=2 \nabla \eta
$$


Therefore by (1.8),

$$
q_{1}=q_{2}+\Delta \eta+4 \frac{\partial \eta}{\partial z} \frac{\partial \eta}{\partial \bar{z}}+2 \frac{\partial \eta}{\partial z} A_{2}+2 \frac{\partial \eta}{\partial \bar{z}} B_{2} .
$$

The operator $L_{1}(x, D)$ given by $(1.10)$ has the Laplace operator as the principal part, the coefficient of $\frac{\partial}{\partial x_{1}}$ is $A_{2}+B_{2}+2 \frac{\partial \eta}{\partial x_{2}}$, the coefficient of $\frac{\partial}{\partial x_{2}}$ is $i\left(B_{2}-A_{2}\right)+$ $2 \frac{\partial \eta}{\partial x_{1}}$, and the coefficient of the zero order term is given by the right-hand side of (1.12). By (1.7) we have $\left.\frac{\partial \eta}{\partial \nu}\right|_{\widetilde{\Gamma}}=0$ and $\left.\eta\right|_{\widetilde{\Gamma}}=\mathcal{C}$ where the function $\mathcal{C}(x)$ is constant on each connected component of $\widetilde{\Gamma}$.

Let us show that the function $\eta$ is continuous. Suppose that it is discontinuous, say, along the curve $\Sigma_{j}$. Let $u_{2} \in H^{1}(\Omega)$ be a solution to the boundary value problem

$$
L_{2}(x, D) u_{2}=0 \quad \text { in } \Omega,\left.\quad u_{2}\right|_{\Gamma_{0}}=0 .
$$

Assume in addition that $u_{2}$ is not identically zero on $\Sigma_{j}$. Let $\widetilde{\Gamma}_{1}$ be one connected component of the set $\widetilde{\Gamma}$ and let $\left.\mathcal{C}\right|_{\widetilde{\Gamma}_{1}}=\widehat{C}$. Without loss of generality we may assume that $\widehat{C}=0$. Indeed if $\widehat{C} \neq 0$, then we replace $\eta$ by the function $\eta-\widehat{C}$. Since the partial Cauchy data of the operators $L_{1}(x, D)$ and $L_{2}(x, D)$ are the same, there exists a solution $u_{1}$ to the boundary value problem

$$
L_{1}(x, D) u_{1}=0 \quad \text { in } \Omega, \quad u_{1}=u_{2} \quad \text { on } \partial \Omega, \quad \frac{\partial u_{1}}{\partial \nu}=\frac{\partial u_{2}}{\partial \nu} \quad \text { on } \widetilde{\Gamma} .
$$

Then the function $v=e^{-\eta} u_{2}$ satisfies

$$
L_{1}(x, D) v=0 \quad \text { in } \Omega^{0},\left.\quad v\right|_{\Gamma_{0}}=0 .
$$

Since $\eta=\frac{\partial \eta}{\partial \nu}=0$ on $\widetilde{\Gamma}_{1}$, we have $v \equiv u_{1}$. However $u_{1} \in H^{1}(\Omega)$ and $v$ is discontinuous along one part of $\Sigma_{j}$. Thus we arrive at a contradiction.

Let us show that $\mathcal{C} \equiv 0$. Suppose that there exists another connected component $\widetilde{\Gamma}_{2}$ of the set $\widetilde{\Gamma}$ such that $\left.\mathcal{C}\right|_{\widetilde{\Gamma}_{2}} \neq 0$. Suppose that the functions $u_{1}, u_{2}$ satisfy (1.13) and (1.14) and $\left.u_{1}\right|_{\widetilde{\Gamma}_{2}}$ is not identically zero.

Then the function $v=e^{-\eta} u_{2}$ satisfies

$$
L_{1}(x, D) v=0 \quad \text { in } \Omega,\left.\quad v\right|_{\Gamma_{0}}=0 .
$$

Moreover, since $\eta=\frac{\partial \eta}{\partial \nu}=0$ on $\widetilde{\Gamma}_{1}$, we have

$$
v=u_{1}, \quad \frac{\partial v}{\partial \nu}=\frac{\partial u_{1}}{\partial \nu} \quad \text { on } \widetilde{\Gamma}_{1}
$$

By the uniqueness for the Cauchy problem for second order elliptic equations, we have $v \equiv u_{1}$. In particular $v=u_{1}$ on $\widetilde{\Gamma}_{2}$. Since $u_{1}=u_{2}$ on $\partial \Omega$, this implies that $\left.e^{-\eta}\right|_{\widetilde{\Gamma}_{2}}=1$. We have arrived at a contradiction. 
There are two specifically interesting cases of $\mathbf{g}=I$ : the magnetic Schrödinger operator and the Laplacian with convection terms. We first apply our result to the case of the magnetic Schrödinger operator. Denote $\widetilde{A}=\left(\widetilde{A}_{1}, \widetilde{A}_{2}\right)$, where $\widetilde{A}_{j}$ are real-valued, $\widetilde{\mathcal{A}}=\widetilde{A}_{1}-i \widetilde{A}_{2}, \operatorname{rot} \widetilde{A}=\frac{\partial \widetilde{A}_{2}}{\partial x_{1}}-\frac{\partial \widetilde{A}_{1}}{\partial x_{2}}, D_{k}=\frac{1}{i} \frac{\partial}{\partial x_{k}}$. Consider the magnetic Schrödinger operator

$$
\mathcal{L}_{\widetilde{A}, \widetilde{q}}(x, D)=\sum_{k=1}^{2}\left(D_{k}+\widetilde{A}_{k}\right)^{2}+\widetilde{q} .
$$

Let us define the following set of partial Cauchy data:

$$
\widetilde{C}_{\widetilde{A}, \widetilde{q}}=\left\{\left(\left.u\right|_{\widetilde{\Gamma}},\left.\frac{\partial u}{\partial \nu}\right|_{\widetilde{\Gamma}}\right) \mid \mathcal{L}_{\widetilde{A}, \widetilde{q}}(x, D) u=0 \text { in } \Omega,\left.u\right|_{\Gamma_{0}}=0, u \in H^{1}(\Omega)\right\} .
$$

For the case of full data in two dimensions, it is known that there is a gauge invariance in this problem and we can recover at best the curl of the magnetic field [26]. The same is valid for the three-dimensional case with partial Cauchy data [12]. We prove here that the converse holds in two dimensions.

Corollary 1.2. Let real-valued vector fields $\widetilde{A}^{(1)}, \widetilde{A}^{(2)} \in C^{5+\alpha}(\bar{\Omega})$ and complexvalued potentials $\widetilde{q}^{(1)}, \widetilde{q}^{(2)} \in C^{4+\alpha}(\bar{\Omega})$ with some $\alpha \in(0,1)$ satisfy $\widetilde{C}_{\widetilde{A}^{(1)}, \widetilde{q}^{(1)}}=$ $\widetilde{C}_{\widetilde{A}^{(2)}, \widetilde{q}^{(2)}}$. Then $\widetilde{q}^{(1)}=\widetilde{q}^{(2)}$, $\operatorname{rot} \widetilde{A}^{(1)}=\operatorname{rot} \widetilde{A}^{(2)}$ in $\Omega$ and $\widetilde{A}^{(1)}=\widetilde{A}^{(2)}$ on $\widetilde{\Gamma}$.

Proof. Straightforward calculations give

$$
\begin{aligned}
\mathcal{L}_{\widetilde{A}, \widetilde{q}}(x, D) & =-\Delta+\frac{2}{i} \widetilde{A}_{1} \frac{\partial}{\partial x_{1}}+\frac{2}{i} \widetilde{A}_{2} \frac{\partial}{\partial x_{2}}+|\widetilde{A}|^{2}+\frac{1}{i} \frac{\partial \widetilde{A}_{1}}{\partial x_{1}}+\frac{1}{i} \frac{\partial \widetilde{A}_{2}}{\partial x_{2}}+\widetilde{q} \\
& =-\Delta+\frac{2}{i} \underset{\widetilde{\mathcal{A}}}{\overline{\partial z}}+\frac{\partial}{i} \widetilde{\mathcal{A}} \frac{\partial}{\partial \bar{z}}+\frac{2}{i} \frac{\partial \widetilde{\mathcal{A}}}{\partial z}-\operatorname{rot} \widetilde{A}+|\widetilde{A}|^{2}+\widetilde{q} .
\end{aligned}
$$

Then the operator $\mathcal{L}_{\widetilde{A}, \widetilde{q}}(x, D)$ is a particular case of (1.1) with the metric $g=I$, $A=-\frac{1}{i} \overline{\widetilde{\mathcal{A}}}, B=-\frac{1}{i} \widetilde{\mathcal{A}}, q=-\left(\frac{2}{i} \frac{\partial \overline{\widetilde{\mathcal{A}}}}{\partial z}-\operatorname{rot} \widetilde{A}+|\widetilde{A}|^{2}+\widetilde{q}\right)$. Suppose that the Schrödinger operators with the vector fields $\widetilde{A}^{(1)}, \widetilde{A}^{(2)}$ and the potentials $\widetilde{q}^{(1)}, \widetilde{q}^{(2)}$ have the same partial Cauchy data. Then (1.8) gives

$$
\operatorname{rot} \widetilde{A}^{(1)}-\operatorname{rot} \widetilde{A}^{(2)}+\widetilde{q}^{(2)}-\widetilde{q}^{(1)} \equiv 0
$$

and (1.9) gives

$$
\begin{aligned}
& \frac{2}{i} \frac{\partial \widetilde{\mathcal{A}}^{(1)}}{\partial \bar{z}}-\frac{2}{i} \frac{\partial \widetilde{\mathcal{A}}^{(2)}}{\partial \bar{z}}-\frac{2}{i} \frac{\partial \overline{\widetilde{\mathcal{A}}^{(1)}}}{\partial z}+\frac{2}{i} \frac{\partial \overline{\widetilde{\mathcal{A}}^{(2)}}}{\partial z}+\operatorname{rot} \widetilde{A}^{(1)} \\
& -\operatorname{rot} \widetilde{A}^{(2)}+\widetilde{q}^{(2)}-\widetilde{q}^{(1)} \equiv 0 .
\end{aligned}
$$


Using the identity $\frac{2}{i} \frac{\partial \mathcal{A}}{\partial \bar{z}}-\frac{2}{i} \frac{\partial \overline{\mathcal{A}}}{\partial z}=-2 \operatorname{rot} \widetilde{A}$, we transform (1.17) to

$$
-\left(\operatorname{rot} \widetilde{A}^{(1)}-\operatorname{rot} \widetilde{A}^{(2)}\right)+\widetilde{q}^{(2)}-\widetilde{q}^{(1)} \equiv 0,
$$

completing the proof.

There is another way to define partial Cauchy data for the Schrödinger operator:

$$
\begin{array}{r}
\widehat{C}_{\widetilde{A}, \widetilde{q}}=\left\{\left(\left.u\right|_{\widetilde{\Gamma}},\left.\left(\frac{\partial u}{\partial \nu}+i(\widetilde{A}, \nu) u\right)\right|_{\widetilde{\Gamma}}\right) \mid \mathcal{L}_{\widetilde{A}, \widetilde{q}}(x, D) u=0 \text { in } \Omega\right. \\
\left.\left.u\right|_{\Gamma_{0}}=0, u \in H^{1}(\Omega)\right\}
\end{array}
$$

Corollary 1.3. Let real-valued vector fields $\widetilde{A}^{(1)}, \widetilde{A}^{(2)} \in C^{5+\alpha}(\bar{\Omega})$ and complexvalued potentials $\widetilde{q}^{(1)}, \widetilde{q}^{(2)} \in C^{4+\alpha}(\bar{\Omega})$ with some $\alpha \in(0,1)$ satisfy $\widehat{C}_{\widetilde{A}^{(1)}, \widetilde{q}^{(1)}}=$ $\widehat{C}_{\widetilde{A}^{(2)}, \widetilde{q}^{(2)}}$. Then $\widetilde{q}^{(1)}=\widetilde{q}^{(2)}$, and $\operatorname{rot} \widetilde{A}^{(1)}=\operatorname{rot} \widetilde{A}^{(2)}$ in $\Omega$.

Proof. Suppose that there exist two vector fields and potentials $\left(\widetilde{A}^{(j)}, \widetilde{q}^{(j)}\right)$ such that $\widehat{C}_{\widetilde{A}^{(1)}, \widetilde{q}^{(1)}}=\widehat{C}_{\widetilde{A}^{(2)}, \widetilde{q}^{(2)}}$. Consider a complex-valued function $\eta \in C^{6+\alpha}(\bar{\Omega})$ with $\left.\eta\right|_{\widetilde{\Gamma}}=0$ such that $i \int_{\widetilde{\Gamma}} \nu \cdot\left(\widetilde{A}^{(1)}-\widetilde{A}^{(2)}\right) d \vec{\tau}=-\frac{1}{i} \frac{\partial \eta}{\partial \nu}$ on $\widetilde{\Gamma}$. Here $\int_{\widetilde{\Gamma}} \cdots d \vec{\tau}$ is a line integral and we set $\vec{\tau}(x)=\left(\nu_{2}(x),-\nu_{1}(x)\right)$. Then $\widetilde{C}_{\widetilde{A}^{(1)}, \widetilde{q}^{(1)}}=\widetilde{C}_{\widetilde{A}^{(2)}+i \nabla \eta, \widetilde{q}^{(2)}}$. Applying Corollary 1.2 , we finish the proof.

Even with data on the whole boundary, no uniqueness result has been published before and Corollaries 1.2 and 1.3 are new. In two dimensions, Sun [26] proved that by measurements on the whole boundary, uniqueness modulo rotations holds, assuming that both the magnetic potential and the electric potential are small. Kang and Uhlmann [17] proved such global uniqueness for the case of measurements on the whole boundary for a special case of the magnetic Schrödinger equation, with the Pauli Hamiltonian. In dimensions $\geq 3$, such global uniqueness was shown in [22] for the case of full data. The regularity assumptions in these results were improved in [23] and [24]. The case of partial data was considered in [12], based on the methods of [18] and [8], and the regularity condition on the coefficients in [19] was improved.

Our main theorem implies that the partial Cauchy data can uniquely determine any two of $(A, B, q)$. First we can prove that $A$ and $B$ are uniquely determined if $q$ is known. Consider the operator

$$
\widetilde{L}(x, D) u=\Delta u+a(x) \frac{\partial u}{\partial x_{1}}+b(x) \frac{\partial u}{\partial x_{2}}+q(x) u .
$$


Here $a, b, q$ are complex-valued functions. Let us define the following set of partial Cauchy data:

$$
\begin{array}{r}
\widetilde{C}_{a, b, q}=\left\{\left(\left.u\right|_{\widetilde{\Gamma}},\left.\frac{\partial u}{\partial \nu}\right|_{\widetilde{\Gamma}}\right) \mid \Delta u+a(x) \frac{\partial u}{\partial x_{1}}+b(x) \frac{\partial u}{\partial x_{2}}+q(x) u=0 \text { in } \Omega,\right. \\
\left.\left.u\right|_{\Gamma_{0}}=0, u \in H^{1}(\Omega)\right\} .
\end{array}
$$

Corollary 1.4. Let $\alpha \in(0,1)$ and fix $q \in C^{4+\alpha}(\bar{\Omega})$. Suppose that two pairs of complex-valued coefficients $\left(a^{(1)}, b^{(1)}\right) \in C^{5+\alpha}(\bar{\Omega}) \times C^{5+\alpha}(\bar{\Omega})$ and $\left(a^{(2)}, b^{(2)}\right) \in$ $C^{5+\alpha}(\bar{\Omega}) \times C^{5+\alpha}(\bar{\Omega})$ satisfy $\widetilde{C}_{a^{(1)}, b^{(1)}, q}=\widetilde{C}_{a^{(2)}, b^{(2)}, q}$. Then $\left(a^{(1)}, b^{(1)}\right) \equiv\left(a^{(2)}, b^{(2)}\right)$.

Proof. Taking into account that $\frac{\partial}{\partial x_{1}}=\frac{\partial}{\partial z}+\frac{\partial}{\partial \bar{z}}$ and $\frac{\partial}{\partial x_{2}}=i\left(\frac{\partial}{\partial z}-\frac{\partial}{\partial \bar{z}}\right)$, we can rewrite the operator (1.18) in the form

$$
\widetilde{L}(x, D) u=\Delta u+(a(x)+i b(x)) \frac{\partial u}{\partial z}+(a(x)-i b(x)) \frac{\partial u}{\partial \bar{z}}+q(x) u .
$$

For the pairs $\left(a^{(1)}, b^{(1)}\right)$ and $\left(a^{(2)}, b^{(2)}\right)$, suppose that the corresponding operators defined by (1.18) have the same partial Cauchy data. Denote $2 A_{k}(x)=a^{(k)}(x)+$ $i b^{(k)}(x)$ and $2 B_{k}(x)=a^{(k)}(x)-i b^{(k)}(x)$. By (1.8), we have

$$
\begin{array}{ll}
-2 \frac{\partial}{\partial z}\left(A_{1}-A_{2}\right)-\left(B_{1}-B_{2}\right) A_{1}-\left(A_{1}-A_{2}\right) B_{2}=0 & \text { in } \Omega \\
-2 \frac{\partial}{\partial \bar{z}}\left(B_{1}-B_{2}\right)-\left(A_{1}-A_{2}\right) B_{1}-\left(B_{1}-B_{2}\right) A_{2}=0 & \text { in } \Omega .
\end{array}
$$

Applying the operator $2 \frac{\partial}{\partial \bar{z}}$ to (1.19) and $2 \frac{\partial}{\partial z}$ to (1.20), we have

$$
\begin{array}{ll}
-\Delta\left(A_{1}-A_{2}\right)-2 \frac{\partial}{\partial \bar{z}}\left(\left(B_{1}-B_{2}\right) A_{1}+\left(A_{1}-A_{2}\right) B_{2}\right)=0 & \text { in } \Omega, \\
-\Delta\left(B_{1}-B_{2}\right)-2 \frac{\partial}{\partial z}\left(\left(A_{1}-A_{2}\right) B_{1}+\left(B_{1}-B_{2}\right) A_{2}\right)=0 & \text { in } \Omega .
\end{array}
$$

By (1.7),

$$
\left.\left(A_{1}-A_{2}\right)\right|_{\widetilde{\Gamma}}=\left.\left(B_{1}-B_{2}\right)\right|_{\widetilde{\Gamma}}=0 .
$$

Using these identities and (1.19) and (1.20), we obtain

$$
\left.\frac{\partial\left(A_{1}-A_{2}\right)}{\partial \nu}\right|_{\widetilde{\Gamma}}=\left.\frac{\partial\left(B_{1}-B_{2}\right)}{\partial \nu}\right|_{\widetilde{\Gamma}}=0 .
$$

The uniqueness of the Cauchy problem for the system (1.21)-(1.22) can be proved in the standard way by using a Carleman estimate (see, e.g., [14]). Hence $A_{1}=A_{2}$ and $B_{1}=B_{2}$ in $\Omega$. 
We remark that Corollary 1.4 generalizes the result of [11] where the uniqueness is obtained assuming that the measurements are made on the whole boundary. In dimension three or more, the global uniqueness was shown in [10] for the case of full data.

Similarly to Corollary 1.4, we can prove that the partial Cauchy data can uniquely determine the potential $q$ and one of the coefficients $A$ and $B$ in (1.1).

Corollary 1.5. For $j=1,2$, let $\left(A_{j}, B_{j}, q_{j}\right) \in C^{5+\alpha}(\bar{\Omega}) \times C^{5+\alpha}(\bar{\Omega}) \times C^{4+\alpha}(\bar{\Omega})$ for some $\alpha \in(0,1)$ and be complex-valued. Assume either $A_{1}=A_{2}$ or $B_{1}=B_{2}$ in $\Omega$. Then $\mathcal{C}_{I, A_{1}, B_{1}, q_{1}}=\mathcal{C}_{I, A_{2}, B_{2}, q_{2}}$ implies $\left(A_{1}, B_{1}, q_{1}\right)=\left(A_{2}, B_{2}, q_{2}\right)$.

Corollaries 1.4 and 1.5 mean that the partial Cauchy data on $\widetilde{\Gamma}$ uniquely determine any two of the three coefficients of a second order elliptic operator whose principal part is the Laplacian.

The proof of Theorem 1.1 uses isothermal coordinates, the Carleman estimate obtained in Section 2, and Theorem 1.3. In this case we need to prove a new Carleman estimate with degenerate harmonic weights in order to construct appropriate complex geometrical optics solutions. The new form of these solutions considerably complicates the arguments, and in particular asymptotic expansions are needed to analyze the behavior of the solutions. In Section 2 we prove the Carleman estimate we need. In Section 3 we state the estimates and asymptotics which are used in the construction of complex geometrical optics solutions. This construction is performed in Section 4. The proof of Theorem 1.3 is completed in Section 5. In Section 6 we prove Theorems 1.1 and 1.2. In Sections 7 and 8 we discuss some technical lemmata needed in the previous sections.

\section{§2. Carleman estimate}

Notations. Throughout the paper we use the following notations: $i=\sqrt{-1}$, $x_{1}, x_{2}, \xi_{1}, \xi_{2} \in \mathbb{R}, z=x_{1}+i x_{2}, \zeta=\xi_{1}+i \xi_{2}, \bar{z}$ denotes the complex conjugate of $z \in \mathbb{C}, D_{k}=\frac{1}{i} \frac{\partial}{\partial x_{k}}, \beta=\left(\beta_{1}, \beta_{2}\right)$ where $\beta_{j} \in \mathbb{N}_{+}=\{0,1,2, \ldots\}$. We identify $x=\left(x_{1}, x_{2}\right) \in \mathbb{R}^{2}$ with $z=x_{1}+i x_{2} \in \mathbb{C}$, and $\zeta=\xi_{1}+i \xi_{2}$ with $\xi=\left(\xi_{1}, \xi_{2}\right)$. We set $\partial_{z}=\frac{\partial}{\partial z}=\frac{1}{2}\left(\frac{\partial}{\partial x_{1}}-i \frac{\partial}{\partial x_{2}}\right), \partial_{\bar{z}}=\frac{\partial}{\partial \bar{z}}=\frac{1}{2}\left(\frac{\partial}{\partial x_{1}}+i \frac{\partial}{\partial x_{2}}\right)$, and $\mathcal{O}_{\epsilon}=\{x \in \Omega \mid$ $\operatorname{dist}(x, \partial \Omega) \leq \epsilon\}$. Moreover we use both notations $\partial_{z}$ and $\frac{\partial}{\partial z}$ etc., and for example we denote $\partial_{\bar{z}}^{2}=\frac{\partial^{2}}{\partial \bar{z}^{2}}$. We say that a function $a(x)$ is antiholomorphic in $\Omega$ if $\left.\partial_{z} a(x)\right|_{\Omega} \equiv 0$. The tangential derivative on the boundary is given by $\frac{\partial}{\partial \vec{\tau}}=\nu_{2} \frac{\partial}{\partial x_{1}}-$ $\nu_{1} \frac{\partial}{\partial x_{2}}$, where $\nu=\left(\nu_{1}, \nu_{2}\right)$ is the unit outer normal to $\partial \Omega$.

The ball of radius $\delta$ centered at $\widehat{x}$ is denoted by $B(\widehat{x}, \delta)=\left\{x \in \mathbb{R}^{2}\right.$ $|x-\widehat{x}|<\delta\}$. The corresponding sphere is denoted by $S(\widehat{x}, \delta)=\left\{x \in \mathbb{R}^{2} \mid\right.$ $|x-\widehat{x}|=\delta\}$. 
If $f: \mathbb{R}^{2} \rightarrow \mathbb{R}^{1}$ is a function, then $f^{\prime \prime}$ is the Hessian matrix with entries $\frac{\partial^{2} f}{\partial x_{i} \partial x_{j}}$. By $(u, v)$ we denote the scalar product of complex-valued functions $u, v$ in $L^{2}(\Omega):(u, v)_{L^{2}(\Omega)}=\int_{\Omega} u \bar{v} d x$, and we set $(u, v)_{L^{2}(\Gamma)}=\int_{\Gamma} u \bar{v} d \vec{\tau}$, where $\Gamma \subset \partial \Omega$ is a subboundary and the integral is taken counterclockwise. Let $\|\cdot\|_{H^{k, \tau}(\Omega)}^{2}=$ $\|\cdot\|_{H^{k}(\Omega)}^{2}+|\tau|^{2 k}\|\cdot\|_{L^{2}(\Omega)}^{2}$ be the norm in the standard Sobolev space with inner product given by $(\cdot, \cdot)_{H^{k, \tau}(\Omega)}=(\cdot, \cdot)_{H^{k}(\Omega)}+|\tau|^{2 k}(\cdot, \cdot)_{L^{2}(\Omega)}$. For any positive function $d$ we introduce the space $L_{d}^{2}(\Omega)=\left\{v(\cdot) \mid\|v\|_{L_{d}^{2}(\Omega)}=\left(\int_{\Omega} d|v|^{2} d x\right)^{1 / 2}<\infty\right\}$.

$\mathcal{L}(X, Y)$ denotes the Banach space of all bounded linear operators from a Banach space $X$ to another Banach space $Y$. By $o_{X}\left(1 / \tau^{\kappa}\right)$ we denote a function $f(\tau, \cdot)$ such that $\|f(\tau, \cdot)\|_{X}=o\left(1 / \tau^{\kappa}\right)$ as $|\tau| \rightarrow+\infty$. Finally for any $\widetilde{x} \in \partial \Omega$ we introduce the left and right tangential derivatives as follows:

$$
\mathbf{D}_{+}(\widetilde{x}) f=\lim _{s \rightarrow+0} \frac{f(\ell(s))-f(\widetilde{x})}{s},
$$

where $\ell(0)=\widetilde{x}, \ell(s)$ is a parametrization of $\partial \Omega$ near $\widetilde{x}, s$ is the length of the curve, and we are moving clockwise as $s$ increases;

$$
\mathbf{D}_{-}(\widetilde{x}) f=\lim _{s \rightarrow-0} \frac{f(\widetilde{\ell}(s))-f(\widetilde{x})}{s},
$$

where $\tilde{\ell}(0)=\widetilde{x}, \widetilde{\ell}(s)$ is the parametrization of $\partial \Omega$ near $\widetilde{x}, s$ is the length of the curve, and we are moving counterclockwise as $s$ increases.

For some $\alpha \in(0,1)$ we consider a function $\Phi(z)=\varphi\left(x_{1}, x_{2}\right)+i \psi\left(x_{1}, x_{2}\right) \in$ $C^{6+\alpha}(\bar{\Omega})$ with real-valued $\varphi$ and $\psi$ such that

$$
\frac{\partial \Phi}{\partial \bar{z}}(z)=0 \quad \text { in } \Omega,\left.\quad \operatorname{Im} \Phi\right|_{\Gamma_{0}^{*}}=0
$$

where $\Gamma_{0}^{*}$ is an open set on $\partial \Omega$ such that $\Gamma_{0} \subset \subset \Gamma_{0}^{*}$. Denote by $\mathcal{H}$ the set of all the critical points of the function $\Phi$ :

$$
\mathcal{H}=\left\{z \in \bar{\Omega} \mid \frac{\partial \Phi}{\partial z}(z)=0\right\} .
$$

Assume that $\Phi$ has no critical points on $\overline{\widetilde{\Gamma}}$, and that all critical points are nondegenerate:

$$
\mathcal{H} \cap \partial \Omega \subset \Gamma_{0}, \quad \frac{\partial^{2} \Phi}{\partial z^{2}}(z) \neq 0 \quad \forall z \in \mathcal{H} .
$$

Then $\Phi$ has only a finite number of critical points and we can set

$$
\mathcal{H} \backslash \Gamma_{0}=\left\{\widetilde{x}_{1}, \ldots, \widetilde{x}_{\ell}\right\}, \quad \mathcal{H} \cap \Gamma_{0}=\left\{\widetilde{x}_{\ell+1}, \ldots, \widetilde{x}_{\ell+\ell^{\prime}}\right\} .
$$

The following proposition was proved in [15]. 
Proposition 2.1. Let $\widetilde{x} \in \Omega$. There exists a sequence $\left\{\Phi_{\epsilon}\right\}_{\epsilon \in(0,1)}$ of functions satisfying (2.1) such that all the critical points of $\Phi_{\epsilon}$ are nondegenerate and there exists a sequence $\left\{\widetilde{x}_{\epsilon}\right\}_{\epsilon \in(0,1)}$ such that

$$
\widetilde{x}_{\epsilon} \in \mathcal{H}_{\epsilon}=\left\{z \in \bar{\Omega} \mid \frac{\partial \Phi_{\epsilon}}{\partial z}(z)=0\right\}, \quad \widetilde{x}_{\epsilon} \rightarrow \widetilde{x} \quad \text { as } \epsilon \rightarrow+0 .
$$

Moreover for any $j$ from $\{1, \ldots, \mathcal{N}\}$ we have

$$
\begin{gathered}
\mathcal{H}_{\epsilon} \cap \gamma_{j}=\emptyset \quad \text { if } \gamma_{j} \cap \widetilde{\Gamma} \neq \emptyset, \\
\mathcal{H}_{\epsilon} \cap \gamma_{j} \subset \Gamma_{0} \quad \text { if } \gamma_{j} \cap \widetilde{\Gamma}=\emptyset, \\
\operatorname{Im} \Phi_{\epsilon}\left(\widetilde{x}_{\epsilon}\right) \notin\left\{\operatorname{Im} \Phi_{\epsilon}(x) \mid x \in \mathcal{H}_{\epsilon} \backslash\left\{\widetilde{x_{\epsilon}}\right\}\right\} \quad \text { and } \quad \operatorname{Im} \Phi_{\epsilon}\left(\widetilde{x}_{\epsilon}\right) \neq 0 .
\end{gathered}
$$

In order to prove (1.7) we need the following proposition, where "card" stands for cardinality.

Proposition 2.2. Let $\widehat{\Gamma}_{*} \subset \subset \widetilde{\Gamma}$ be an arc with left endpoint $x_{-}$and right endpoint $x_{+}$, and be oriented clockwise. For any $\widehat{x} \in \operatorname{Int} \widehat{\Gamma}_{*}$ there exists a function $\Phi(z)$ which satisfies (2.1), (2.2), $\left.\operatorname{Im} \Phi\right|_{\partial \Omega \backslash \widehat{\Gamma}_{*}}=0$,

$$
\widehat{x} \in \mathcal{G}=\left\{x \in \widehat{\Gamma}_{*} \mid \frac{\partial \operatorname{Im} \Phi}{\partial \vec{\tau}}(x)=0\right\}, \quad \operatorname{card} \mathcal{G}<\infty
$$

and

$$
\left(\frac{\partial}{\partial \vec{\tau}}\right)^{2} \operatorname{Im} \Phi(x) \neq 0 \quad \forall x \in \mathcal{G} \backslash\left\{x_{-}, x_{+}\right\}
$$

Moreover

$$
\operatorname{Im} \Phi(\widehat{x}) \neq \operatorname{Im} \Phi(x) \quad \forall x \in \mathcal{G} \backslash\{\widehat{x}\} \quad \text { and } \quad \operatorname{Im} \Phi(\widehat{x}) \neq 0
$$

and

$$
\mathbf{D}_{+}\left(x_{-}\right)\left(\frac{\partial}{\partial \vec{\tau}}\right)^{6} \operatorname{Im} \Phi \neq 0, \quad \mathbf{D}_{-}\left(x_{+}\right)\left(\frac{\partial}{\partial \vec{\tau}}\right)^{6} \operatorname{Im} \Phi \neq 0 .
$$

Proof. Denote $\widehat{\Gamma}_{0}^{*}=\partial \Omega \backslash \widehat{\Gamma}_{*}$. Let $\widehat{x}_{-}, \widehat{x}_{+} \in \partial \Omega$ be such that the arc $\left[\widehat{x}_{-}, \widehat{x}_{+}\right]$is contained in $\left(x_{-}, x_{+}\right)$and $\widehat{x} \in\left(\widehat{x}_{-}, \widehat{x}_{+}\right)$. Let $x_{0}$ be another fixed point from $\left(\widehat{x}, \widehat{x}_{+}\right)$. We claim that there exists a pair $\left(\varphi_{0}, \psi_{0}\right) \in C^{6+\alpha}(\bar{\Omega}) \times C^{6+\alpha}(\bar{\Omega})$ which solves the system of the Cauchy-Riemann equations in $\Omega$ such that
(A) $\left.\psi_{0}\right|_{\widehat{\Gamma}_{0}^{*}}=0,\left.\left|\frac{\partial \varphi_{0}}{\partial \vec{\tau}}\right|\right|_{\gamma_{j} \backslash \widehat{\Gamma}_{*}}>0$ if $\gamma_{j} \cap \widehat{\Gamma}_{*} \neq \emptyset ; \frac{\partial \psi_{0}}{\partial \vec{\tau}}(\widehat{x})=0,\left(\frac{\partial}{\partial \vec{\tau}}\right)^{2} \psi_{0}(\widehat{x}) \neq 0$, $\mathbf{D}_{+}\left(x_{-}\right)\left(\frac{\partial}{\partial \vec{\tau}}\right)^{6} \psi_{0} \neq 0, \mathbf{D}_{-}\left(x_{+}\right)\left(\frac{\partial}{\partial \vec{\tau}}\right)^{6} \psi_{0} \neq 0$,

(B) the restriction of the function $\psi_{0}$ to the $\operatorname{arc}\left(\widehat{x}_{-}, \widehat{x}_{+}\right)$is a Morse function,

(C) $\frac{\partial \psi_{0}}{\partial \vec{\tau}}>0$ on $\left(x_{-}, \widehat{x}_{-}\right], \frac{\partial \psi_{0}}{\partial \vec{\tau}}<0$ on $\left[\widehat{x}_{+}, x_{+}\right)$, 
(D) $\psi_{0}(\widehat{x}) \notin\left\{\psi_{0}(x) \mid x \in \partial \Omega \backslash\{\widehat{x}\}, \frac{\partial \psi_{0}}{\partial \vec{\tau}}(x)=0\right\}$,

(E) if $\gamma_{j} \cap \widehat{\Gamma}_{*}=\emptyset$, then the restriction of the function $\varphi_{0}$ to $\gamma_{j}$ has only two nondegenerate critical points.

Such a pair of functions may be constructed in the following way. Let $\gamma_{1} \cap \widehat{\Gamma}_{*}$ $\neq \emptyset$ and $\gamma_{j} \cap \widehat{\Gamma}_{*}=\emptyset$ for all $j \in\{2, \ldots, \mathcal{N}\}$. First, by Corollary 7.1 in Appendix I, for some $\alpha \in(0,1)$ there exists a solution $(\widetilde{\varphi}, \widetilde{\psi}) \in C^{6+\alpha}(\bar{\Omega}) \times C^{6+\alpha}(\bar{\Omega})$ to the Cauchy-Riemann equations satisfying

$$
\left.\widetilde{\psi}\right|_{\partial \Omega \backslash\left[x_{0}, \widehat{x}_{+}\right]}=\psi_{*},\left.\quad \frac{\partial \widetilde{\varphi}}{\partial \vec{\tau}}\right|_{\gamma_{1} \backslash\left[x_{0}, \widehat{x}_{+}\right]}<\beta<0
$$

and such that the function $\widetilde{\varphi}$ has only two nondegenerate critical points on $\gamma_{j}$ if $\gamma_{j} \cap \widehat{\Gamma}_{*}=\emptyset$. The function $\psi_{*}$ has the following properties: $\left.\psi_{*}\right|_{\widehat{\Gamma}_{0}^{*}}=0, \frac{\partial \psi_{*}}{\partial \vec{\tau}}>0$ on $\left(x_{-}, \widehat{x}_{-}\right], \frac{\partial \psi_{*}}{\partial \vec{\tau}}<0$ on $\left[\widehat{x}_{+}, x_{+}\right), \mathbf{D}_{+}\left(x_{-}\right)\left(\frac{\partial}{\partial \bar{\tau}}\right)^{6} \psi_{*} \neq 0, \mathbf{D}_{-}\left(x_{+}\right)\left(\frac{\partial}{\partial \vec{\tau}}\right)^{6} \psi_{*} \neq 0$. The function $\psi_{*}$ on $\left[\widehat{x}_{-}, x_{0}\right]$ has only one critical point $\widehat{x}$ and $\psi_{*}(\widehat{x}) \neq 0$. On $\left(x_{0}, \widehat{x}_{+}\right)$, the Cauchy data are not fixed. The restriction of $\widetilde{\psi}$ to $\left[x_{0}, \widehat{x}_{+}\right]$can be approximated in $C^{6+\alpha}\left(\left[x_{0}, \widehat{x}_{+}\right]\right)$by a sequence $\left\{g_{\epsilon}\right\}_{\epsilon \in(0,1)}$ of Morse functions such that

$$
\left(\frac{\partial}{\partial \vec{\tau}}\right)^{k} \widetilde{\psi}(x)=\left(\frac{\partial}{\partial \vec{\tau}}\right)^{k} g_{\epsilon}(x), \quad x \in\left\{\widehat{x}_{+}, x_{0}\right\}, k \in\{0,1, \ldots, 6\},
$$

and

$$
\psi_{*}(\widehat{x}) \notin\left\{g_{\epsilon}(x) \mid \frac{\partial g_{\epsilon}(x)}{\partial \vec{\tau}}=0\right\} .
$$

Let $\mathcal{J} \subset \subset\left(x_{-}, \widehat{x}_{-}\right)$be an arc. We have

$$
\frac{\partial \widetilde{\psi}}{\partial \vec{\tau}}>\beta^{\prime}>0 \quad \text { on } \mathcal{J} \text { for some positive } \beta^{\prime} .
$$

Let $\left(\varphi_{\epsilon}, \psi_{\epsilon}\right) \in C^{6+\alpha}(\bar{\Omega}) \times C^{6+\alpha}(\bar{\Omega})$ be a solution to the Cauchy-Riemann equations with boundary data $\psi_{\epsilon}=0$ on $\partial \Omega \backslash\left(\mathcal{J} \cup\left[x_{0}, \widehat{x}_{+}\right]\right)$and $\psi_{\epsilon}=g_{\epsilon}-\widetilde{\psi}$ on $\left[x_{0}, \widehat{x}_{+}\right]$, and on $\mathcal{J}$ the Cauchy data are chosen in such a way that

$$
\left\|\psi_{\epsilon}\right\|_{C^{6+\alpha}(\partial \Omega)}+\left\|\varphi_{\epsilon}\right\|_{C^{6+\alpha}(\partial \Omega)} \rightarrow 0 \quad \text { as } \quad\left\|g_{\epsilon}-\widetilde{\psi}\right\|_{C^{6+\alpha}\left(\left[x_{0}, \widehat{x}_{+}\right]\right)} \rightarrow 0 .
$$

By (2.8) and (2.9), for all small positive $\epsilon$, the restriction of $\widetilde{\psi}+\psi_{\epsilon}$ to $\partial \Omega$ satisfies

$$
\begin{aligned}
& \left.\left(\widetilde{\psi}+\psi_{\epsilon}\right)\right|_{\widehat{\Gamma}_{0}^{*}}=0,\left.\quad \frac{\partial\left(\widetilde{\psi}+\psi_{\epsilon}\right)}{\partial \nu}\right|_{\gamma_{1} \backslash\left[x_{0}, \widehat{x}_{+}\right]}<0, \quad \frac{\partial\left(\widetilde{\psi}+\psi_{\epsilon}\right)}{\partial \vec{\tau}}>0 \text { on }\left(x_{-}, \widehat{x}_{-}\right], \\
& \frac{\partial\left(\widetilde{\psi}+\psi_{\epsilon}\right)}{\partial \vec{\tau}}<0 \text { on }\left[\widehat{x}_{+}, x_{+}\right),\left.\quad\left(\widetilde{\psi}+\psi_{\epsilon}\right)\right|_{\left[x_{0}, \widehat{x}_{+}\right]}=g_{\epsilon},\left.\quad\left(\widetilde{\psi}+\psi_{\epsilon}\right)\right|_{\left[\widehat{x}_{-}, x_{0}\right]}=\psi_{*}, \\
& \mathbf{D}_{+}\left(x_{-}\right)\left(\frac{\partial}{\partial \vec{\tau}}\right)^{6}\left(\widetilde{\psi}+\psi_{\epsilon}\right) \neq 0, \quad \mathbf{D}_{-}\left(x_{+}\right)\left(\frac{\partial}{\partial \vec{\tau}}\right)^{6}\left(\widetilde{\psi}+\psi_{\epsilon}\right) \neq 0 .
\end{aligned}
$$


If $j \geq 2$, then the restriction of $\varphi_{\epsilon}+\widetilde{\varphi}$ to $\gamma_{j}$ has only two critical points on $\gamma_{j} \subset \widehat{\Gamma}_{0}^{*}$. These critical points are nondegenerate if $\epsilon$ is sufficiently small.

Therefore the restriction of $\widetilde{\psi}+\psi_{\epsilon}$ to $\widehat{\Gamma}_{*}$ has a finite number of critical points. Some of them may be critical points of $\widetilde{\psi}+\psi_{\epsilon}$ considered as a function on $\bar{\Omega}$. We change $\widetilde{\psi}+\psi_{\epsilon}$ slightly so that all of its critical points are in $\Omega \cup \widehat{\Gamma}_{0}^{*}$. Suppose that $\widetilde{\psi}+\psi_{\epsilon}$ has critical points on $\widehat{\Gamma}_{*}$. Then they should be critical points of $g_{\epsilon}$, otherwise it is the point $\widehat{x}$. We denote these points by $\widehat{x}_{1}, \ldots, \widehat{x}_{m}$. Let $(\widehat{\varphi}, \widehat{\psi}) \in$ $C^{6+\alpha}(\bar{\Omega}) \times C^{6+\alpha}(\bar{\Omega})$ be a solution to the Cauchy-Riemann problem (7.1) with the following boundary data:

$$
\left.\widehat{\psi}\right|_{\partial \Omega \backslash \mathcal{J}}=0,\left.\quad\left|\frac{\partial \widehat{\psi}}{\partial \nu}\right|\right|_{\overline{\gamma_{1} \backslash \mathcal{J}}}>0 .
$$

For all small positive $\epsilon_{1}$ the function $\widetilde{\psi}+\psi_{\epsilon}+\epsilon_{1} \widehat{\psi}$ has no critical points on $\widetilde{\Gamma}$ and its restriction to $\widehat{\Gamma}_{*}$ has a finite number of nondegenerate critical points. Therefore the pairs $\left(\widetilde{\varphi}+\varphi_{\epsilon}+\epsilon_{1} \widehat{\varphi}, \widetilde{\psi}+\psi_{\epsilon}+\epsilon_{1} \widehat{\psi}\right)$ satisfy $(\mathrm{A})-(\mathrm{E})$.

For a pair $\left(\varphi_{0}, \psi_{0}\right)$ satisfying $(\mathrm{A})-(\mathrm{E})$ the function $\varphi_{0}+i \psi_{0}$ satisfies all the hypotheses of Proposition 2.2 except that some of its critical points may possibly be degenerate. In order to fix this problem we consider the following perturbation of $\varphi_{0}+i \psi_{0}$. By Proposition 4.2 in [15], there exists a holomorphic function $w$ in $\Omega$ such that

$$
\left.\operatorname{Im} w\right|_{\Gamma_{0}^{*}}=0,\left.\quad w\right|_{\mathcal{H}_{0}}=\left.\frac{\partial w}{\partial z}\right|_{\mathcal{H}_{0}}=0,\left.\quad \frac{\partial^{2} w}{\partial z^{2}}\right|_{\mathcal{H}_{0}} \neq 0
$$

where $\mathcal{H}_{0}$ is the set of all critical points of $\varphi_{0}+i \psi_{0}$. Denote $\Phi_{\delta}=\varphi_{0}+i \psi_{0}+\delta w$. For all sufficiently small positive $\delta$, we have

$$
\mathcal{H}_{0} \subset \mathcal{H}_{\delta} \equiv\left\{\begin{array}{l|l|l}
x \in \Omega & \frac{\partial}{\partial z} \Phi_{\delta}(x)=0
\end{array}\right\} .
$$

We now show that for all sufficiently small positive $\delta$, all critical points of $\Phi_{\delta}$ are nondegenerate. Let $\widetilde{x}$ be a critical point of $\varphi_{0}+i \psi_{0}$. If $\widetilde{x}$ is nondegenerate, then by the implicit function theorem, there exists a ball $B\left(\widetilde{x}, \delta_{1}\right)$ in which $\Phi_{\delta}$ has only one nondegenerate critical point for all small $\delta$. Let $\widetilde{x}$ be a degenerate critical point of $\varphi_{0}+i \psi_{0}$. Without loss of generality we may assume that $\widetilde{x}=0$. In some neighborhood of 0 , we have $\frac{\partial \Phi_{\delta}}{\partial z}=\sum_{k=1}^{\infty} c_{k} z^{k+\widehat{k}}-\delta \sum_{k=1}^{\infty} b_{k} z^{k}$ for some natural number $\widehat{k}$ and some $c_{1} \neq 0$. Moreover (2.10) implies $b_{1} \neq 0$. Let $\left(x_{1, \delta}, x_{2, \delta}\right) \in \mathcal{H}_{\delta}$ and $z_{\delta}=x_{1, \delta}+i x_{2, \delta} \rightarrow 0$. Then either

$$
z_{\delta}=0 \quad \text { or } \quad z_{\delta}^{\widehat{k}}=\delta b_{1} / c_{1}+o(\delta) \quad \text { as } \delta \rightarrow 0 .
$$

Therefore $\frac{\partial^{2} \Phi_{\delta}}{\partial z^{2}}\left(z_{\delta}\right) \neq 0$ for all sufficiently small $\delta$. 
Let $\alpha \in(0,1)$ and let $\mathcal{A}, \mathcal{B} \in C^{6+\alpha}(\bar{\Omega})$ be two complex-valued solutions to the boundary value problem

$$
2 \frac{\partial \mathcal{A}}{\partial \bar{z}}=-A \quad \text { in } \Omega,\left.\quad \operatorname{Im} \mathcal{A}\right|_{\Gamma_{0}}=0, \quad 2 \frac{\partial \mathcal{B}}{\partial z}=-B \quad \text { in } \Omega,\left.\quad \operatorname{Im} \mathcal{B}\right|_{\Gamma_{0}}=0 .
$$

Consider the boundary value problem

$$
\frac{\partial a}{\partial \bar{z}}=0 \quad \text { in } \Omega, \quad \frac{\partial d}{\partial z}=0 \quad \text { in } \Omega,\left.\quad\left(a e^{\mathcal{A}}+d e^{\mathcal{B}}\right)\right|_{\Gamma_{0}}=\beta .
$$

The existence of such functions $a(z)$ and $d(\bar{z})$ is given by the following proposition.

Proposition 2.3. Let $\alpha \in(0,1)$, and let $\mathcal{A}$ and $\mathcal{B}$ be as in (2.12). If $\beta \in C^{5+\alpha}\left(\bar{\Gamma}_{0}\right)$, then $(2.13)$ has at least one solution $(a, d) \in C^{5+\alpha}(\bar{\Omega}) \times C^{5+\alpha}(\bar{\Omega})$ such that

$$
\|(a, d)\|_{C^{5+\alpha}(\bar{\Omega}) \times C^{5+\alpha}(\bar{\Omega})} \leq C_{1}\|\beta\|_{C^{5+\alpha}\left(\bar{\Gamma}_{0}\right)} .
$$

If $\beta \in H^{1 / 2}\left(\Gamma_{0}\right)$, then (2.13) has at least one solution $(a, d) \in H^{1}(\Omega) \times H^{1}(\Omega)$ such that

$$
\|(a, d)\|_{H^{1}(\Omega) \times H^{1}(\Omega)} \leq C_{2}\|\beta\|_{H^{1 / 2}\left(\Gamma_{0}\right)} .
$$

Proof. Let $\widetilde{\Omega}$ be a domain in $\mathbb{R}^{2}$ with smooth boundary such that $\Omega \subset \widetilde{\Omega}$ and there exists an open subdomain $\widetilde{\Gamma}_{0} \subset \partial \widetilde{\Omega}$ satisfying $\bar{\Gamma}_{0} \subset \widetilde{\Gamma}_{0}$. Denote $\Gamma^{*}=\partial \widetilde{\Omega} \backslash \widetilde{\Gamma}_{0}$. We extend $\mathcal{A}, \mathcal{B}$ to $\widetilde{\Gamma}_{0}$ keeping the regularity and we extend $\beta$ to $\widetilde{\Gamma}_{0}$ in such a way that $\|\beta\|_{H^{1 / 2}\left(\widetilde{\Gamma}_{0}\right)} \leq C_{3}\|\beta\|_{H^{1 / 2}\left(\Gamma_{0}\right)}$ or $\|\beta\|_{C^{5+\alpha}\left(\widetilde{\Gamma}_{0}\right)} \leq C_{3}\|\beta\|_{C^{5+\alpha}\left(\overline{\Gamma_{0}}\right)}$ where the constant $C_{3}$ is independent of $\beta$. By the trace theorem there exist a constant $C_{4}$, independent of $\beta$, and a pair $(r, \widetilde{r})$ such that $\left.\left(r e^{\mathcal{A}}+\widetilde{r} e^{\mathcal{B}}\right)\right|_{\widetilde{\Gamma}_{0}}=\beta$ and if $\beta \in H^{1 / 2}\left(\widetilde{\Gamma}_{0}\right)$ then $(r, \widetilde{r}) \in H^{1}(\Omega) \times H^{1}(\Omega)$ and

$$
\|(r, \widetilde{r})\|_{H^{1}(\Omega) \times H^{1}(\Omega)} \leq C_{4}\|\beta\|_{H^{1 / 2}\left(\Gamma_{0}\right)} .
$$

Similarly if $\beta \in C^{5+\alpha}\left(\widetilde{\Gamma}_{0}\right)$ then $(r, \widetilde{r}) \in C^{5+\alpha}(\bar{\Omega}) \times C^{5+\alpha}(\bar{\Omega})$ and

$$
\|(r, \widetilde{r})\|_{C^{5+\alpha}(\bar{\Omega}) \times C^{5+\alpha}(\bar{\Omega})} \leq C_{5}\|\beta\|_{C^{5+\alpha}\left(\Gamma_{0}\right)} .
$$

Let $f=\frac{\partial r}{\partial z}$ and $\tilde{f}=\frac{\partial \widetilde{r}}{\partial \bar{z}}$. For any $\epsilon$ from $(0,1)$, consider the extremal problem $J_{\epsilon}(p, \widetilde{p})=\|(p, \widetilde{p})\|_{L^{2}(\widetilde{\Omega})}^{2}+\frac{1}{\epsilon}\left\|\frac{\partial p}{\partial z}-f\right\|_{L^{2}(\widetilde{\Omega})}^{2}+\frac{1}{\epsilon}\left\|\frac{\partial \widetilde{p}}{\partial \bar{z}}-\widetilde{f}\right\|_{L^{2}(\widetilde{\Omega})}^{2} \rightarrow \inf , \quad(p, \widetilde{p}) \in \mathcal{K}$, where $\mathcal{K}=\left\{\left(h_{1}, h_{2}\right) \in L^{2}(\widetilde{\Omega}) \times L^{2}(\widetilde{\Omega})\left|\left(h_{1} e^{\mathcal{A}}+h_{2} e^{\mathcal{B}}\right)\right|_{\widetilde{\Gamma}_{0}}=0\right\}$. Denote the solution to this extremal problem by $\left(p_{\epsilon}, \widetilde{p}_{\epsilon}\right)$. Then

$$
J_{\epsilon}^{\prime}\left(p_{\epsilon}, \tilde{p}_{\epsilon}\right)(\delta, \widetilde{\delta})=0 \quad \forall(\delta, \widetilde{\delta}) \in \mathcal{K}
$$


Hence

$$
\begin{aligned}
\left(\left(p_{\epsilon}, \widetilde{p}_{\epsilon}\right),(\delta, \widetilde{\delta})\right)_{L^{2}(\widetilde{\Omega})}+ & \frac{1}{\epsilon}\left(\frac{\partial p_{\epsilon}}{\partial z}-f, \frac{\partial \delta}{\partial z}\right)_{L^{2}(\widetilde{\Omega})} \\
& +\frac{1}{\epsilon}\left(\frac{\partial \widetilde{p}_{\epsilon}}{\partial \bar{z}}-\widetilde{f}, \frac{\partial \widetilde{\delta}}{\partial \bar{z}}\right)_{L^{2}(\widetilde{\Omega})}=0 \quad \forall(\delta, \widetilde{\delta}) \in \mathcal{K} .
\end{aligned}
$$

Denote $P_{\epsilon}=-\frac{1}{\epsilon}\left(\frac{\partial p_{\epsilon}}{\partial z}-f\right), \widetilde{P}_{\epsilon}=-\frac{1}{\epsilon}\left(\frac{\partial \widetilde{p}_{\epsilon}}{\partial \bar{z}}-\widetilde{f}\right)$. From (2.16) we obtain

$$
\begin{aligned}
& \frac{\partial P_{\epsilon}}{\partial \bar{z}}=p_{\epsilon}, \quad \frac{\partial \widetilde{P}_{\epsilon}}{\partial z}=\widetilde{p}_{\epsilon},\left.\quad P_{\epsilon}\right|_{\Gamma^{*}}=\left.\widetilde{P}_{\epsilon}\right|_{\Gamma^{*}}=0, \\
& \left.\left(\left(\nu_{1}+i \nu_{2}\right) P_{\epsilon} e^{\mathcal{B}}-\left(\nu_{1}-i \nu_{2}\right) \widetilde{P}_{\epsilon} e^{\mathcal{A}}\right)\right|_{\widetilde{\Gamma}_{0}}=0 .
\end{aligned}
$$

We claim that there exists a constant $C_{6}$ independent of $\epsilon$ such that

$$
\left\|\left(P_{\epsilon}, \widetilde{P}_{\epsilon}\right)\right\|_{H^{1}(\widetilde{\Omega})} \leq C_{6}\left(\left\|\left(p_{\epsilon}, \widetilde{p}_{\epsilon}\right)\right\|_{L^{2}(\widetilde{\Omega})}+\left\|\left(P_{\epsilon}, \widetilde{P}_{\epsilon}\right)\right\|_{L^{2}(\widetilde{\Omega})}\right) .
$$

It clearly suffices to prove (2.18) locally assuming that $\operatorname{supp}\left(p_{\epsilon}, \tilde{p}_{\epsilon}\right)$ is in a small neighborhood of zero and the vector $(0,1)$ is orthogonal to $\partial \Omega$ on the intersection of this neighborhood with the boundary. Using a conformal transformation we may assume that $\partial \Omega \cap \operatorname{supp} P_{\epsilon}, \partial \Omega \cap \operatorname{supp} \widetilde{P}_{\epsilon} \subset\left\{x_{1}=0\right\}$. In order to prove (2.18) we consider the system of equations

$$
\frac{\partial \mathbf{u}}{\partial x_{2}}+\widehat{B} \frac{\partial \mathbf{u}}{\partial x_{1}}=\mathbf{F}, \quad \operatorname{supp} \mathbf{u} \subset B(0, \delta) \cap\left\{x \mid x_{2} \geq 0\right\} .
$$

Here

$$
\begin{aligned}
& \mathbf{u}=\left(u_{1}, u_{2}, u_{3}, u_{4}\right)=\left(\operatorname{Re} P_{\epsilon}, \operatorname{Im} P_{\epsilon}, \operatorname{Re} \widetilde{P}_{\epsilon}, \operatorname{Im} \widetilde{P}_{\epsilon}\right), \\
& \mathbf{F}=2\left(\operatorname{Re} p_{\epsilon}, \operatorname{Im} p_{\epsilon}, \operatorname{Re} \widetilde{p}_{\epsilon}, \operatorname{Im} \widetilde{p}_{\epsilon}\right), \quad \widehat{B}=\left(\begin{array}{cccc}
0 & 1 & 0 & 0 \\
-1 & 0 & 0 & 0 \\
0 & 0 & 0 & -1 \\
0 & 0 & 1 & 0
\end{array}\right) .
\end{aligned}
$$

The matrix $\widehat{B}$ has two eigenvalues $\pm i$ and four linearly independent eigenvectors:

$$
\begin{array}{ll}
\mathbf{q}_{3}=(0,0,1, i), \mathbf{q}_{4}=(1,-i, 0,0) & \text { corresponding to the eigenvalue }-i, \\
\mathbf{q}_{1}=(1, i, 0,0), \mathbf{q}_{2}=(0,0,1,-i) & \text { corresponding to the eigenvalue } i .
\end{array}
$$

We set $\mathbf{r}_{1}=\left(\nu_{1} e^{\mathcal{B}},-\nu_{2} e^{\mathcal{B}},-\nu_{1} e^{\mathcal{A}},-\nu_{2} e^{\mathcal{A}}\right), \mathbf{r}_{2}=\left(\nu_{2} e^{\mathcal{B}}, \nu_{1} e^{\mathcal{B}}, \nu_{2} e^{\mathcal{A}},-\nu_{1} e^{\mathcal{A}}\right)$. Consider the matrix $D=\left\{d_{j \ell}\right\}$ where $d_{j \ell}=\mathbf{r}_{j} \cdot \mathbf{q}_{\ell}, 1 \leq j, \ell \leq 2$. We have

$$
D=\left(\begin{array}{cc}
\left(\nu_{1}-i \nu_{2}\right) e^{\mathcal{B}} & -\left(\nu_{1}-i \nu_{2}\right) e^{\mathcal{A}} \\
\left(\nu_{2}+i \nu_{1}\right) e^{\mathcal{B}} & \left(\nu_{2}+i \nu_{1}\right) e^{\mathcal{A}}
\end{array}\right) .
$$

Since the Lopatinski determinant $\operatorname{det} D$ is not zero we obtain (2.18) (see e.g. [30]). 
Next we need to get rid of the second term on the right-hand side of (2.18). Suppose that for any $\widetilde{C}$ one can find $\epsilon$ such that the estimate

$$
\left\|\left(P_{\epsilon}, \widetilde{P}_{\epsilon}\right)\right\|_{H^{1}(\widetilde{\Omega})} \leq \widetilde{C}\left\|\left(p_{\epsilon}, \widetilde{p}_{\epsilon}\right)\right\|_{L^{2}(\widetilde{\Omega})}
$$

fails. That is, there exist a sequence $\epsilon_{k} \rightarrow 0$ and a sequence $\left\{C_{\epsilon_{k}}\right\}$ such that $\lim _{\epsilon_{k} \rightarrow+0} C_{\epsilon_{k}}=+\infty$ and

$$
\left\|\frac{\left(p_{\epsilon_{k}}, \widetilde{p}_{\epsilon_{k}}\right)}{\left\|\left(P_{\epsilon_{k}}, \widetilde{P}_{\epsilon_{k}}\right)\right\|_{H^{1}(\widetilde{\Omega})}}\right\|_{L^{2}(\widetilde{\Omega})}<\frac{1}{C_{\epsilon_{k}}} .
$$

Then we define $\left(Q_{\epsilon_{k}}, \widetilde{Q}_{\epsilon_{k}}\right)=\left(P_{\epsilon_{k}}, \widetilde{P}_{\epsilon_{k}}\right) /\left\|\left(P_{\epsilon_{k}}, \widetilde{P}_{\epsilon_{k}}\right)\right\|_{H^{1}(\widetilde{\Omega})}$ and $\left(q_{\epsilon_{k}}, \widetilde{q}_{\epsilon_{k}}\right)=$ $\left(p_{\epsilon_{k}}, \widetilde{\rho}_{\epsilon_{k}}\right) /\left\|\left(P_{\epsilon_{k}}, \widetilde{P}_{\epsilon_{k}}\right)\right\|_{H^{1}(\widetilde{\Omega})}$. Consequently, $\left\|\left(q_{\epsilon_{k}}, \widetilde{q}_{\epsilon_{k}}\right)\right\|_{L^{2}(\widetilde{\Omega})} \rightarrow 0$ as $\epsilon_{k} \rightarrow 0$. Passing to the limit in (2.17) we have

$$
\frac{\partial Q}{\partial \bar{z}}=0 \quad \text { in } \widetilde{\Omega}, \quad \frac{\partial \widetilde{Q}}{\partial z}=0 \quad \text { in } \widetilde{\Omega},\left.\quad Q\right|_{\Gamma^{*}}=\left.\widetilde{Q}\right|_{\Gamma^{*}}=0 .
$$

By the uniqueness for the Cauchy problem for the operator $\partial_{z}$ we conclude $Q \equiv \widetilde{Q} \equiv 0$. On the other hand, since $\left\|\left(Q_{\epsilon_{k}}, \widetilde{Q}_{\epsilon_{k}}\right)\right\|_{H^{1}(\widetilde{\Omega})}=1$, we can extract a subsequence (not relabeled) which is convergent in $L^{2}(\widetilde{\Omega})$. Therefore the sequence $\left\{\left(Q_{\epsilon_{k}}, \widetilde{Q}_{\epsilon_{k}}\right)\right\}$ converges to zero in $L^{2}(\widetilde{\Omega}) \times L^{2}(\widetilde{\Omega})$. By (2.18), we have $1 / C_{7} \leq$ $\left\|\left(q_{\epsilon_{k}}, \widetilde{q}_{\epsilon_{k}}\right)\right\|_{L^{2}(\widetilde{\Omega})}+\left\|\left(Q_{\epsilon_{k}}, \widetilde{Q}_{\epsilon_{k}}\right)\right\|_{L^{2}(\widetilde{\Omega})}$. Therefore $\liminf \epsilon_{\epsilon_{k} \rightarrow 0}\left\|\left(Q_{\epsilon_{k}}, \widetilde{Q}_{\epsilon_{k}}\right)\right\|_{L^{2}(\widetilde{\Omega})} \neq 0$, and this is a contradiction. Hence

$$
\left\|\left(P_{\epsilon_{k}}, \widetilde{P}_{\epsilon_{k}}\right)\right\|_{H^{1}(\widetilde{\Omega})} \leq C_{8}\left\|\left(p_{\epsilon_{k}}, \widetilde{p}_{\epsilon_{k}}\right)\right\|_{L^{2}(\widetilde{\Omega})} \quad \forall \epsilon>0 .
$$

Let us plug in $(2.16)$ the function $\left(p_{\epsilon_{k}}, \widetilde{p}_{\epsilon_{k}}\right)$ instead of $(\delta, \widetilde{\delta})$. Then, by the above inequality, in view of the definitions of $P_{\epsilon_{k}}$ and $\widetilde{P}_{\epsilon_{k}}$, we have

$$
\begin{aligned}
\left\|\left(p_{\epsilon_{k}}, \widetilde{p}_{\epsilon_{k}}\right)\right\|_{L^{2}(\widetilde{\Omega})}^{2} & \leq C_{9}\left((f, \widetilde{f}),\left(P_{\epsilon_{k}}, \widetilde{P}_{\epsilon_{k}}\right)\right)_{L^{2}(\widetilde{\Omega})} \leq C_{10}\|(f, \widetilde{f})\|_{L^{2}(\widetilde{\Omega})}\left\|\left(P_{\epsilon_{k}}, \widetilde{P}_{\epsilon_{k}}\right)\right\|_{L^{2}(\widetilde{\Omega})} \\
& \leq C_{11}\|(f, \widetilde{f})\|_{L^{2}(\widetilde{\Omega})}\left\|\left(p_{\epsilon_{k}}, \widetilde{p}_{\epsilon_{k}}\right)\right\|_{L^{2}(\widetilde{\Omega})} .
\end{aligned}
$$

This inequality implies that the sequence $\left(p_{\epsilon_{k}}, \widetilde{p}_{\epsilon_{k}}\right)$ is bounded in $L^{2}(\widetilde{\Omega})$ and

$$
\left(\frac{\partial p_{\epsilon_{k}}}{\partial z}, \frac{\partial{\widetilde{\omega_{k}}}_{\epsilon_{k}}}{\partial \bar{z}}\right) \rightarrow(f, \widetilde{f}) \quad \text { in } L^{2}(\widetilde{\Omega}) \times L^{2}(\widetilde{\Omega}) .
$$

Then we construct a solution to (2.13) such that

$$
\|(p, \widetilde{p})\|_{L^{2}(\widetilde{\Omega})} \leq C_{12}\|(f, \widetilde{f})\|_{L^{2}(\widetilde{\Omega})} .
$$

Observe that we can write the boundary value problem

$$
\frac{\partial p}{\partial z}=f \quad \text { in } \Omega, \quad \frac{\partial \widetilde{p}}{\partial \bar{z}}=\widetilde{f} \quad \text { in } \Omega,\left.\quad\left(p e^{\mathcal{A}}+\widetilde{p} e^{\mathcal{B}}\right)\right|_{\widetilde{\Gamma}_{0}}=0
$$


in the form of $(2.19)$ with $\mathbf{u}=(\operatorname{Re} p, \operatorname{Im} p, \operatorname{Re} \widetilde{p}, \operatorname{Im} \widetilde{p}), \mathbf{F}=2(\operatorname{Re} f, \operatorname{Im} f, \operatorname{Re} \widetilde{f}, \operatorname{Im} \widetilde{f})$. We set $\mathbf{r}_{1}=\left(e^{\mathcal{A}},-e^{\mathcal{A}},-e^{\mathcal{B}},-e^{\mathcal{B}}\right)$ and $\mathbf{r}_{2}=\left(e^{\mathcal{A}}, e^{\mathcal{A}}, e^{\mathcal{B}},-e^{\mathcal{B}}\right)$. Consider the matrix $D=\left\{d_{j \ell}\right\}$ where $d_{j \ell}=\mathbf{r}_{j} \cdot \mathbf{q}_{\ell}, 1 \leq j, \ell \leq 2$. We have

$$
D=\left(\begin{array}{cc}
e^{\mathcal{B}} & -e^{\mathcal{A}} \\
e^{\mathcal{B}} & e^{\mathcal{A}}
\end{array}\right) .
$$

Since $\operatorname{det} D \neq 0$, estimate (2.20) implies (2.14) and (2.15) (see, e.g., [30, Theorem 4.1.2]).

The following proposition was proved in [15] in the case where the partial differential equations have no zeroth order terms, but in our case the proof is similar:

Proposition 2.4. Let $\Phi$ satisfy (2.1), (2.2), $\tau \in \mathbb{R}$, and let $C=C_{1}+i C_{2}$ belong to $C^{1}(\bar{\Omega})$ where $C_{1}, C_{2}$ are real-valued. Let $\widetilde{f} \in L^{2}(\Omega)$, and let $\widetilde{v} \in H^{1}(\Omega)$ be a solution to

$$
2 \frac{\partial}{\partial z} \widetilde{v}-\tau \frac{\partial \Phi}{\partial z} \widetilde{v}+C \widetilde{v}=\widetilde{f} \quad \text { in } \Omega
$$

or to

$$
2 \frac{\partial}{\partial \bar{z}} \widetilde{v}-\tau \frac{\partial \bar{\Phi}}{\partial \bar{z}} \widetilde{v}+C \widetilde{v}=\widetilde{f} \quad \text { in } \Omega
$$

In the case (2.21) we have

$$
\begin{aligned}
\left\|\frac{\partial \widetilde{v}}{\partial x_{1}}-i \operatorname{Im}\left(\tau \frac{\partial \Phi}{\partial z}-C\right) \widetilde{v}\right\|_{L^{2}(\Omega)}^{2}-\int_{\partial \Omega}\left(\tau \frac{\partial \varphi}{\partial \nu}-\left(\nu_{1} C_{1}+\nu_{2} C_{2}\right)\right)|\widetilde{v}|^{2} d \sigma \\
-\int_{\Omega}\left(\frac{\partial C_{1}}{\partial x_{1}}+\frac{\partial C_{2}}{\partial x_{2}}\right)|\widetilde{v}|^{2} d x+\operatorname{Re} \int_{\partial \Omega} i \frac{\partial \widetilde{v}}{\partial \vec{\tau}} \overline{\widetilde{v}} d \sigma \\
+\left\|-\frac{1}{i} \frac{\partial \widetilde{v}}{\partial x_{2}}-\operatorname{Re}\left(\tau \frac{\partial \Phi}{\partial z}-C\right) \widetilde{v}\right\|_{L^{2}(\Omega)}^{2}=\|\widetilde{f}\|_{L^{2}(\Omega)}^{2} .
\end{aligned}
$$

In the case (2.22) we have

$$
\begin{aligned}
\left\|\frac{\partial \widetilde{v}}{\partial x_{1}}-i \operatorname{Im}\left(\tau \frac{\partial \bar{\Phi}}{\partial \bar{z}}-C\right) \widetilde{v}\right\|_{L^{2}(\Omega)}-\int_{\partial \Omega}\left(\tau \frac{\partial \varphi}{\partial \nu}-\left(\nu_{1} C_{1}-\nu_{2} C_{2}\right)\right)|\widetilde{v}|^{2} d \sigma \\
-\int_{\Omega}\left(\frac{\partial C_{1}}{\partial x_{1}}-\frac{\partial C_{2}}{\partial x_{2}}\right)|\widetilde{v}|^{2} d x-\operatorname{Re} \int_{\partial \Omega} i \frac{\partial \widetilde{v}}{\partial \vec{\tau}} \overline{\widetilde{v}} d \sigma \\
+\left\|\frac{1}{i} \frac{\partial \widetilde{v}}{\partial x_{2}}-\operatorname{Re}\left(\tau \frac{\partial \bar{\Phi}}{\partial \bar{z}}-C\right) \widetilde{v}\right\|_{L^{2}(\Omega)}^{2}=\|\widetilde{f}\|_{L^{2}(\Omega)}^{2} .
\end{aligned}
$$


Consider the boundary value problem

$$
\left\{\begin{array}{l}
\mathcal{K}(x, D) u=\left(4 \frac{\partial}{\partial z} \frac{\partial}{\partial \bar{z}}+2 A \frac{\partial}{\partial z}+2 B \frac{\partial}{\partial \bar{z}}\right) u=f \quad \text { in } \Omega \\
\left.u\right|_{\partial \Omega}=0
\end{array}\right.
$$

For this problem we have the following Carleman estimate with boundary terms.

Proposition 2.5. Suppose that $\Phi$ satisfies (2.1), (2.2), $u \in H_{0}^{1}(\Omega)$ and $\|A\|_{L^{\infty}(\Omega)}+$ $\|B\|_{L^{\infty}(\Omega)} \leq K$. Then there exist $\tau_{0}=\tau_{0}(K, \Phi)$ and $C_{13}=C_{13}(K, \Phi)$ independent of $u$ and $\tau$ such that for all $|\tau|>\tau_{0}$,

$$
\begin{aligned}
|\tau|\left\|u e^{\tau \varphi}\right\|_{L^{2}(\Omega)}^{2}+ & \left\|u e^{\tau \varphi}\right\|_{H^{1}(\Omega)}^{2}+\left\|\frac{\partial u}{\partial \nu} e^{\tau \varphi}\right\|_{L^{2}\left(\Gamma_{0}\right)}^{2}+\tau^{2}\left\|\left|\frac{\partial \Phi}{\partial z}\right| u e^{\tau \varphi}\right\|_{L^{2}(\Omega)}^{2} \\
& \leq C_{13}\left(\left\|(\mathcal{K}(x, D) u) e^{\tau \varphi}\right\|_{L^{2}(\Omega)}^{2}+|\tau| \int_{\widetilde{\Gamma}^{*}}\left|\frac{\partial u}{\partial \nu}\right|^{2} e^{2 \tau \varphi} d \sigma\right),
\end{aligned}
$$

where $\widetilde{\Gamma}^{*}=\partial \Omega \backslash \Gamma_{0}^{*}$.

Proof. Denote $\widetilde{v}=u e^{\tau \varphi}$ and $\mathcal{K}(x, D) u=f$. Observe that $\varphi\left(x_{1}, x_{2}\right)=\frac{1}{2}(\Phi(z)+$ $\overline{\Phi(z)})$. Therefore

$$
\begin{aligned}
& e^{\tau \varphi} \Delta\left(e^{-\tau \varphi} \widetilde{v}\right)=\left(2 \frac{\partial}{\partial z}-\tau \frac{\partial \Phi}{\partial z}\right)\left(2 \frac{\partial}{\partial \bar{z}}-\tau \frac{\partial \bar{\Phi}}{\partial \bar{z}}\right) \widetilde{v} \\
& =\left(2 \frac{\partial}{\partial \bar{z}}-\tau \frac{\partial \bar{\Phi}}{\partial \bar{z}}\right)\left(2 \frac{\partial}{\partial z}-\tau \frac{\partial \Phi}{\partial z}\right) \widetilde{v}=\widetilde{f}:=\left(f-2 B \frac{\partial u}{\partial \bar{z}}-2 A \frac{\partial u}{\partial z}\right) e^{\tau \varphi} .
\end{aligned}
$$

Assume now that $u$ is a real-valued function. Denote $\widetilde{w}=\left(2 \frac{\partial}{\partial \bar{z}}-\tau \frac{\partial \bar{\Phi}}{\partial \bar{z}}\right) \widetilde{v}$.

Thanks to the zero Dirichlet boundary condition for $u$, we have

$$
\left.\widetilde{w}\right|_{\partial \Omega}=\left.2 \frac{\partial \widetilde{v}}{\partial \bar{z}}\right|_{\partial \Omega}=\left.\left(\nu_{1}+i \nu_{2}\right) \frac{\partial \widetilde{v}}{\partial \nu}\right|_{\partial \Omega} .
$$

Let $\mathcal{C}$ be some smooth, real-valued vector function in $\Omega$ such that

$$
2 \frac{\partial \mathcal{C}}{\partial z}=C(x)=C_{1}(x)+i C_{2}(x) \quad \text { in } \Omega, \quad \operatorname{Im} \mathcal{C}=0 \quad \text { on } \Gamma_{0},
$$

where $\vec{C}=\left(C_{1}, C_{2}\right)$ is a smooth function in $\Omega$ such that

$$
\operatorname{div} \vec{C}=1 \quad \text { in } \Omega, \quad(\nu, \vec{C})=-1 \quad \text { on } \Gamma_{0} .
$$


Let $N>0$ be a parameter. By Proposition 2.4 we have the following integral equality:

$$
\begin{aligned}
& \left\|\frac{\partial\left(\widetilde{w} e^{N \mathcal{C}}\right)}{\partial x_{1}}-i \operatorname{Im}\left(\tau \frac{\partial \bar{\Phi}}{\partial \bar{z}}+N C\right)\left(\widetilde{w} e^{N \mathcal{C}}\right)\right\|_{L^{2}(\Omega)} \\
& -\int_{\partial \Omega}\left(\tau \frac{\partial \varphi}{\partial \nu}+N\left(\nu_{1} C_{1}+\nu_{2} C_{2}\right)\right)\left|\frac{\partial \widetilde{v}}{\partial \nu} e^{N \mathcal{C}}\right|^{2} d \sigma \\
& +N \int_{\Omega}\left|\widetilde{w} e^{N \mathcal{C}}\right|^{2} d x+\operatorname{Re} \int_{\partial \Omega} i \frac{\partial}{\partial \vec{\tau}}\left(\widetilde{w} e^{N \mathcal{C}}\right) \widetilde{\widetilde{w} e^{N \mathcal{C}}} d \sigma \\
& +\left\|-\frac{1}{i} \frac{\partial\left(\widetilde{w} e^{N \mathcal{C}}\right)}{\partial x_{2}}-\operatorname{Re}\left(\tau \frac{\partial \Phi}{\partial z}+N C\right)\left(\widetilde{w} e^{N \mathcal{C}}\right)\right\|_{L^{2}(\Omega)}^{2}=\left\|\widetilde{f} e^{\tau \varphi+N \mathcal{C}}\right\|_{L^{2}(\Omega)}^{2} .
\end{aligned}
$$

We now simplify the integral $\operatorname{Re} i \int_{\partial \Omega} \frac{\partial}{\partial \vec{\tau}}\left(\widetilde{w} e^{N \mathcal{C}}\right) \overline{\widetilde{w}_{1} e^{N \mathcal{C}}} d \sigma$. We recall that $\widetilde{v}=$ $u e^{\tau \varphi}$ in $\Omega$ and $\widetilde{w}=\left(\nu_{1}+i \nu_{2}\right) \frac{\partial \widetilde{v}}{\partial \nu}=\left(\nu_{1}+i \nu_{2}\right) \frac{\partial u}{\partial \nu} e^{\tau \varphi}$ on $\partial \Omega$. Denote $\left(\nu_{1}+i \nu_{2}\right) e^{N i \operatorname{Im} \mathcal{C}}$ $=R+i P$ where $R, P$ are real-valued. Therefore

$$
\begin{aligned}
\operatorname{Re} \int_{\partial \Omega} & i \frac{\partial}{\partial \vec{\tau}}\left(\widetilde{w} e^{N \mathcal{C}}\right) \overline{\widetilde{w} e^{N \mathcal{C}}} d \sigma \\
= & \operatorname{Re} \int_{\partial \Omega} i \frac{\partial}{\partial \vec{\tau}}\left((R+i P) \frac{\partial u}{\partial \nu} e^{\tau \varphi+N \operatorname{Re} \mathcal{C}}\right)(R-i P) \frac{\partial u}{\partial \nu} e^{\tau \varphi+N \operatorname{Re} \mathcal{C}} d \sigma \\
\quad= & \operatorname{Re} \int_{\partial \Omega} i \frac{\partial}{\partial \vec{\tau}}(R+i P)\left|\frac{\partial\left(\widetilde{v} e^{N \mathcal{C}}\right)}{\partial \nu}\right|^{2}(R-i P) d \sigma .
\end{aligned}
$$

Using the above formula in (2.28), we obtain

$$
\begin{aligned}
& \| \frac{\partial\left(\widetilde{w} e^{N \mathcal{C}}\right)}{\partial x_{1}}-\left.i \operatorname{Im}\left(\tau \frac{\partial \bar{\Phi}}{\partial \bar{z}}+N C\right)\left(\widetilde{w} e^{N \mathcal{C}}\right)\right|_{L^{2}(\Omega)} \\
& -\int_{\partial \Omega}\left(\tau \frac{\partial \varphi}{\partial \nu}+N\left(\nu_{1} C_{1}+\nu_{2} C_{2}\right)\right)\left|\frac{\partial \widetilde{v}}{\partial \nu} e^{N \mathcal{C}}\right|^{2} d \sigma \\
& +N \int_{\Omega}\left|\widetilde{w} e^{N \mathcal{C}}\right|^{2} d x+\operatorname{Re} \int_{\partial \Omega} i \frac{\partial}{\partial \vec{\tau}}(R+i P)\left|\frac{\partial\left(\widetilde{v} e^{N \operatorname{Re}}\right)}{\partial \nu}\right|^{2}(R-i P) d \sigma \\
& +\left\|-\frac{1}{i} \frac{\partial\left(\widetilde{w} e^{N \mathcal{C}}\right)}{\partial x_{2}}-\operatorname{Re}\left(\tau \frac{\partial \Phi}{\partial z}+N C\right)\left(\widetilde{w} e^{N \mathcal{C}}\right)\right\|_{L^{2}(\Omega)}^{2}=\left\|\widetilde{f} e^{\tau \varphi+N \mathcal{C}}\right\|_{L^{2}(\Omega)}^{2} .
\end{aligned}
$$

Taking a positive parameter $N$ sufficiently large and taking into account that the function $R+i P$ is independent of $N$ on $\Gamma_{0}^{*}$, we conclude from (2.30), (2.27) that

$$
\begin{aligned}
-\int_{\partial \Omega}\left(\tau \frac{\partial \varphi}{\partial \nu}+\frac{N}{2}\left(\nu_{1} C_{1}+\nu_{2} C_{2}\right)\right)\left|\frac{\partial \widetilde{v}}{\partial \nu} e^{N \mathcal{C}}\right|^{2} d \sigma+N \int_{\Omega}\left|\widetilde{w} e^{N \mathcal{C}}\right|^{2} d x \\
\leq\left\|\widetilde{f} e^{\tau \varphi+N \mathcal{C}}\right\|_{L^{2}(\Omega)}^{2}+C(N) \int_{\widetilde{\Gamma}}\left|\frac{\partial \widetilde{v}}{\partial \nu} e^{N \mathcal{C}}\right|^{2} d \sigma .
\end{aligned}
$$


Simple computations give

$$
\begin{aligned}
4\left\|\frac{\partial\left(\widetilde{v} e^{N \operatorname{Re}}\right)}{\partial \bar{z}}\right\|_{L^{2}(\Omega)}^{2} & +\tau^{2}\left\|\frac{\overline{\partial \Phi}}{\partial z}\left(\widetilde{v} e^{N \operatorname{Re} \mathcal{C}}\right)\right\|_{L^{2}(\Omega)}^{2} \\
& =\left\|2 \frac{\partial\left(\widetilde{v} e^{N \operatorname{Re} \mathcal{C}}\right)}{\partial \bar{z}}-\tau \frac{\overline{\partial \Phi}}{\partial z}\left(\widetilde{v} e^{N \operatorname{Re} \mathcal{C}}\right)\right\|_{L^{2}(\Omega)}^{2} \\
& =\left\|e^{N \operatorname{Re} \mathcal{C}}\left(2 \frac{\partial \widetilde{v}}{\partial \bar{z}}-\left(\tau \frac{\overline{\partial \Phi}}{\partial z}+2 \frac{\partial N \operatorname{Re} \mathcal{C}}{\partial \bar{z}}\right) \widetilde{v}\right)\right\|_{L^{2}(\Omega)}^{2} \\
& \leq 2\left\|\widetilde{w} e^{N \mathcal{C}}\right\|_{L^{2}(\Omega)}^{2}+C(N)\left\|u e^{\tau \varphi}\right\|_{L^{2}(\Omega)}^{2} .
\end{aligned}
$$

Since the function $\Phi$ has zeros of at most second order by assumption (2.2), there exists a constant $C_{14}>0$ independent of $\tau$ such that

$$
\tau\left\|\widetilde{v} e^{N \operatorname{Re} \mathcal{C}}\right\|_{L^{2}(\Omega)}^{2} \leq C_{14}\left(\left\|\widetilde{v} e^{N \operatorname{Re} \mathcal{C}}\right\|_{H^{1}(\Omega)}^{2}+\tau^{2}\left\|\left|\frac{\partial \Phi}{\partial z}\right| \widetilde{v} e^{N \operatorname{Re} \mathcal{C}}\right\|_{L^{2}(\Omega)}^{2}\right)
$$

Therefore by (2.31)-(2.33) there exists $N_{0}>0$ such that for any $N>N_{0}$ there exists $\tau_{0}(N)$ such that

$$
\begin{array}{r}
-\int_{\partial \Omega}\left(\tau \frac{\partial \varphi}{\partial \nu}+\frac{N}{2}\left(\nu_{1} C_{1}+\nu_{2} C_{2}\right)\right)\left|\frac{\partial \widetilde{v}}{\partial \nu} e^{N \mathcal{C}}\right|^{2} d \sigma+\frac{N}{2} \int_{\Omega}\left|\widetilde{w} e^{N \mathcal{C}}\right|^{2} d x \\
+\tau\left\|\widetilde{v} e^{N \operatorname{Re} \mathcal{C}}\right\|_{L^{2}(\Omega)}^{2}+\left\|\widetilde{v} e^{N \operatorname{Re} \mathcal{C}}\right\|_{H^{1}(\Omega)}^{2}+\tau^{2}\left\|\left|\frac{\partial \Phi}{\partial z}\right| \widetilde{v} e^{N \operatorname{Re} \mathcal{C}}\right\|_{L^{2}(\Omega)}^{2} \\
\leq\left\|\widetilde{f} e^{\tau \varphi+N \mathcal{C}}\right\|_{L^{2}(\Omega)}^{2}+C_{15}(N) \int_{\widetilde{\Gamma}^{*}}\left|\frac{\partial \widetilde{v}}{\partial \nu} e^{N \mathcal{C}}\right|^{2} d \sigma
\end{array}
$$

for all $\tau>\tau_{0}(N)$.

In order to remove the assumption that $u$ is the real-valued function, we obtain (2.34) separately for the real and imaginary parts of $u$ and combine them. This concludes the proof of the proposition.

As a corollary we derive a Carleman inequality for the function $u$ which satisfies the integral equality

$$
\left(u, \mathcal{K}(x, D)^{*} w\right)_{L^{2}(\Omega)}+(f, w)_{H^{1, \tau}(\Omega)}+\left(g e^{\tau \varphi}, e^{-\tau \varphi} w\right)_{H^{1 / 2, \tau}(\widetilde{\Gamma})}=0
$$

for all $w \in \mathcal{X}=\left\{w \in H^{1}(\Omega)|w|_{\Gamma_{0}}=0, \mathcal{K}(x, D)^{*} w \in L^{2}(\Omega)\right\}$. We have

Corollary 2.1. Suppose that $\Phi$ satisfies (2.1), (2.2), $f \in H^{1}(\Omega), g \in H^{1 / 2}(\widetilde{\Gamma})$, $u \in L^{2}(\Omega)$ and the coefficients $A$ and $B$ of $\mathcal{K}(x, D)$ belong to $\left\{C \in C^{1}(\bar{\Omega})\right.$ | $\left.\|C\|_{C^{1}(\bar{\Omega})} \leq K\right\}$. Then there exist $\tau_{0}=\tau_{0}(K, \Phi)$ and $C_{16}=C_{16}(K, \Phi)$, independent 
of $u$ and $\tau$, such that

$$
\left\|u e^{\tau \varphi}\right\|_{L^{2}(\Omega)}^{2} \leq C_{16}|\tau|\left(\left\|f e^{\tau \varphi}\right\|_{H^{1, \tau}(\Omega)}^{2}+\left\|g e^{\tau \varphi}\right\|_{H^{1 / 2, \tau}(\widetilde{\Gamma})}^{2}\right) \quad \forall|\tau| \geq \tau_{0}
$$

for solutions of (2.35).

Proof. Let $\epsilon$ be some positive number and $d(x)$ be a smooth positive function on $\widetilde{\Gamma}$ which blows up like $1 /|x-y|^{8}$ for any $y \in \partial \widetilde{\Gamma}$. Consider the extremal problem

$$
\begin{aligned}
J_{\epsilon}(w)= & \frac{1}{2}\left\|w e^{-\tau \varphi}\right\|_{L^{2}(\Omega)}^{2}+\frac{1}{2 \epsilon}\left\|\mathcal{K}(x, D)^{*} w-u e^{2 \tau \varphi}\right\|_{L^{2}(\Omega)}^{2} \\
& +\frac{1}{2|\tau|}\left\|w e^{-\tau \varphi}\right\|_{L_{d}^{2}(\widetilde{\Gamma})}^{2} \rightarrow \inf
\end{aligned}
$$

for

$$
w \in \widehat{\mathcal{X}}=\left\{w \in H^{1 / 2}(\Omega)\left|\mathcal{K}(x, D)^{*} w \in L^{2}(\Omega), w\right|_{\Gamma_{0}}=0\right\} .
$$

There exists a unique solution to $(2.37),(2.38)$, which we denote by $\widehat{w}_{\epsilon}$. By Fermat's theorem, we have

$$
J_{\epsilon}^{\prime}\left(\widehat{w}_{\epsilon}\right)[\delta]=0 \quad \forall \delta \in \widehat{\mathcal{X}} .
$$

Using the notation $p_{\epsilon}=\frac{1}{\epsilon}\left(\mathcal{K}(x, D)^{*} \widehat{w}_{\epsilon}-u e^{2 \tau \varphi}\right)$, this implies

$$
\mathcal{K}(x, D) p_{\epsilon}+\widehat{w}_{\epsilon} e^{-2 \tau \varphi}=0 \quad \text { in } \Omega,\left.\quad p_{\epsilon}\right|_{\partial \Omega}=0,\left.\quad \frac{\partial p_{\epsilon}}{\partial \nu}\right|_{\widetilde{\Gamma}}=d \frac{\widehat{w}_{\epsilon}}{|\tau|} e^{-2 \tau \varphi}
$$

By Proposition 2.5 we have

$$
\begin{array}{r}
|\tau|\left\|p_{\epsilon} e^{\tau \varphi}\right\|_{L^{2}(\Omega)}^{2}+\left\|p_{\epsilon} e^{\tau \varphi}\right\|_{H^{1}(\Omega)}^{2}+\left\|\frac{\partial p_{\epsilon}}{\partial \nu} e^{\tau \varphi}\right\|\left\|_{L^{2}\left(\Gamma_{0}\right)}^{2}+\tau^{2}\right\|\left|\frac{\partial \Phi}{\partial z}\right| p_{\epsilon} e^{\tau \varphi} \|_{L^{2}(\Omega)}^{2} \\
\leq C_{11}\left(\left\|\widehat{w}_{\epsilon} e^{-\tau \varphi}\right\|_{L^{2}(\Omega)}^{2}+\frac{1}{|\tau|} \int_{\widetilde{\Gamma}^{*}}\left|\widehat{w}_{\epsilon}\right|^{2} e^{-2 \tau \varphi} d \sigma\right) \leq 2 C_{17} J_{\epsilon}\left(\widehat{w}_{\epsilon}\right)
\end{array}
$$

Substituting in $(2.39) \delta=\widehat{w}_{\epsilon}$, we obtain

$$
2 J_{\epsilon}\left(\widehat{w}_{\epsilon}\right)+\operatorname{Re}\left(u e^{2 \tau \varphi}, p_{\epsilon}\right)_{L^{2}(\Omega)}=0 .
$$

Applying estimate (2.41) to the second term of the above equality, we have

$$
|\tau| J_{\epsilon}\left(\widehat{w}_{\epsilon}\right) \leq C_{18}\left\|u e^{\tau \varphi}\right\|_{L^{2}(\Omega)}^{2} .
$$

Using this estimate, we pass to the limit in (2.40) as $\epsilon$ goes to zero. We obtain

$$
\mathcal{K}(x, D) p+\widehat{w} e^{-2 \tau \varphi}=0 \quad \text { in } \Omega,\left.\quad p\right|_{\partial \Omega}=0,\left.\quad \frac{\partial p}{\partial \nu}\right|_{\widetilde{\Gamma}}=d \frac{\widehat{w}}{|\tau|} e^{-2 \tau \varphi}
$$




$$
\begin{aligned}
& \mathcal{K}(x, D)^{*} \widehat{w}-u e^{2 \tau \varphi}=0 \quad \text { in } \Omega,\left.\quad \widehat{w}\right|_{\Gamma_{0}}=0, \\
& |\tau|\left\|\widehat{w} e^{-\tau \varphi}\right\|_{L^{2}(\Omega)}^{2}+\left\|\widehat{w} e^{-\tau \varphi}\right\|_{L^{2}(\widetilde{\Gamma})}^{2} \leq C_{19}\left\|u e^{\tau \varphi}\right\|_{L^{2}(\Omega)}^{2} .
\end{aligned}
$$

Since $\widehat{w} \in L^{2}(\Omega)$, we have $p \in H^{2}(\Omega)$, and by the trace theorem $\frac{\partial p}{\partial \nu} \in H^{1 / 2}(\partial \Omega)$. The relation $(2.42)$ implies $\widehat{w} \in H^{1 / 2}(\widetilde{\Gamma})$. Since $\widehat{w} \in L_{d}^{2}(\widetilde{\Gamma})$ and $\left.\widehat{w}\right|_{\Gamma_{0}}=0$, we have $\widehat{w} \in H^{1 / 2}(\partial \Omega)$. By (2.41)-(2.44), we obtain

$$
\left\|\widehat{w} e^{-\tau \varphi}\right\|_{H^{1 / 2, \tau}(\partial \Omega)} \leq C_{20}|\tau|^{1 / 2}\left\|u e^{\tau \varphi}\right\|_{L^{2}(\Omega)} .
$$

Taking the scalar product of (2.43) with $\widehat{w} e^{-2 \tau \varphi}$ and using estimates (2.45) and (2.44), we obtain

$$
\begin{aligned}
\frac{1}{|\tau|}\left\|(\nabla \widehat{w}) e^{-\tau \varphi}\right\|_{L^{2}(\Omega)}^{2}+|\tau|\left\|\widehat{w} e^{-\tau \varphi}\right\|_{L^{2}(\Omega)}^{2}+\frac{1}{|\tau|}\left\|\widehat{w} e^{-\tau \varphi}\right\|_{H^{1 / 2, \tau}(\widetilde{\Gamma})}^{2} \\
\leq C_{21}\left\|u e^{\tau \varphi}\right\|_{L^{2}(\Omega)}^{2} .
\end{aligned}
$$

From this estimate and the standard duality argument, the statement of Corollary 2.1 follows immediately.

Consider the problem

$$
\Delta u+2 A \frac{\partial u}{\partial z}+2 B \frac{\partial u}{\partial \bar{z}}+q u=f e^{\tau \varphi} \quad \text { in } \Omega,\left.\quad u\right|_{\Gamma_{0}}=g e^{\tau \varphi} .
$$

Set

$$
L(x, D) u=\Delta u+2 A \frac{\partial u}{\partial z}+2 B \frac{\partial u}{\partial \bar{z}}+q u \quad \text { in } \Omega .
$$

We have

Proposition 2.6. Let $A, B \in C^{5+\alpha}(\bar{\Omega})$ for some $\alpha \in(0,1)$ and $q \in L^{\infty}(\Omega)$. There exists $\tau_{0}>0$ such that for all $|\tau|>\tau_{0}$ there exists a solution to the boundary value problem (2.47) such that

$$
\begin{aligned}
\frac{1}{\sqrt{|\tau|}}\left\|(\nabla u) e^{-\tau \varphi}\right\|_{L^{2}(\Omega)}+\sqrt{|\tau|}\left\|u e^{-\tau \varphi}\right\|_{L^{2}(\Omega)} & \\
& \leq C_{22}\left(\|f\|_{L^{2}(\Omega)}+\|g\|_{H^{1 / 2, \tau}\left(\Gamma_{0}\right)}\right) .
\end{aligned}
$$

Let $\epsilon$ be a sufficiently small positive number. If $\operatorname{supp} f \subset G_{\epsilon}=\{x \in \Omega \mid$ $\operatorname{dist}(x, \mathcal{H})>\epsilon\}$ and $g=0$, then there exists $\tau_{0}>0$ such that for all $|\tau|>\tau_{0}$ there exists a solution to the boundary value problem (2.47) such that

$$
\left\|(\nabla u) e^{-\tau \varphi}\right\|_{L^{2}(\Omega)}+|\tau|\left\|u e^{-\tau \varphi}\right\|_{L^{2}(\Omega)} \leq C_{23}(\epsilon)\|f\|_{L^{2}(\Omega)} .
$$

Proof. First we reduce the problem (2.47) to the case $g=0$. Let $r(z)$ be a holomorphic function and $\widetilde{r}(\bar{z})$ be an antiholomorphic function such that $\left.\left(e^{\mathcal{A}} r+e^{\mathcal{B}} \widetilde{r}\right)\right|_{\Gamma_{0}}=g$ 
where $\mathcal{A}, \mathcal{B} \in C^{6+\alpha}(\bar{\Omega})$ are defined as in (2.12). The existence of $r, \widetilde{r}$ follows from Proposition 2.3 , and they can be chosen such that

$$
\|r\|_{H^{1}(\Omega)}+\|\widetilde{r}\|_{H^{1}(\Omega)} \leq C_{24}\|g\|_{H^{1 / 2}\left(\Gamma_{0}\right)} .
$$

We look for a solution $u$ in the form

$$
u=\left(e^{\mathcal{A}+\tau \Phi} r+e^{\mathcal{B}+\tau \bar{\Phi}} \widetilde{r}\right)+\widetilde{u},
$$

where

$$
\begin{aligned}
& L(x, D) \widetilde{u}=\widetilde{f} e^{\tau \varphi} \quad \text { in } \Omega,\left.\quad \widetilde{u}\right|_{\Gamma_{0}}=0, \\
& \widetilde{f}=f-\left(q-2 \frac{\partial A}{\partial z}-A B\right) e^{\mathcal{A}} r e^{i \tau \psi}-\left(q-2 \frac{\partial B}{\partial \bar{z}}-A B\right) e^{\mathcal{B}} \widetilde{r} e^{-i \tau \psi} .
\end{aligned}
$$

In order to prove (2.48), we consider the extremal problem

$$
\begin{aligned}
\widetilde{I}_{\epsilon}(u)= & \frac{1}{2}\left\|u e^{-\tau \varphi}\right\|_{H^{1, \tau}(\Omega)}^{2}+\frac{1}{2 \epsilon}\left\|L(x, D) u-\widetilde{f} e^{\tau \varphi}\right\|_{L^{2}(\Omega)}^{2} \\
& +\frac{1}{2}\left\|u e^{-\tau \varphi}\right\|_{H^{1 / 2, \tau}(\widetilde{\Gamma})}^{2} \rightarrow \inf
\end{aligned}
$$

for

$$
u \in \mathcal{Y}=\left\{w \in H^{1}(\Omega)|w|_{\Gamma_{0}}=0, L(x, D) w \in L^{2}(\Omega)\right\} .
$$

There exists a unique solution to problem (2.51), (2.52), which we denote by $\widehat{u}_{\epsilon}$. By Fermat's theorem, we have

$$
\widetilde{I}_{\epsilon}^{\prime}\left(\widehat{u}_{\epsilon}\right)[\delta]=0 \quad \forall \delta \in \mathcal{Y} .
$$

Let $p_{\epsilon}=\frac{1}{\epsilon}\left(L(x, D) \widehat{u}_{\epsilon}-\widetilde{f} e^{\tau \varphi}\right)$. Applying Corollary 2.1 we obtain from (2.53)

$$
\begin{aligned}
\frac{1}{|\tau|}\left\|p_{\epsilon} e^{\tau \varphi}\right\|_{L^{2}(\Omega)}^{2} & \leq C_{25}\left(\left\|\widehat{u}_{\epsilon} e^{-\tau \varphi}\right\|_{H^{1, \tau}(\Omega)}^{2}+\left\|\widehat{u}_{\epsilon} e^{-\tau \varphi}\right\|_{H^{1 / 2, \tau}(\widetilde{\Gamma})}^{2}\right) \\
& \leq 2 C_{25} \widetilde{I}_{\epsilon}\left(\widehat{u}_{\epsilon}\right) .
\end{aligned}
$$

Substituting $\delta=\widehat{u}_{\epsilon}$ in (2.53), we obtain

$$
2 \widetilde{I}_{\epsilon}\left(\widehat{u}_{\epsilon}\right)+\operatorname{Re}\left(p_{\epsilon}, \tilde{f} e^{\tau \varphi}\right)_{L^{2}(\Omega)}=0 .
$$

Applying estimate (2.54) to this equality, we have

$$
\widetilde{I}_{\epsilon}\left(\widehat{u}_{\epsilon}\right) \leq C_{26}|\tau|\|\widetilde{f}\|_{L^{2}(\Omega)}^{2} .
$$

Using this estimate, we pass to the limit as $\epsilon \rightarrow+0$. We obtain

$$
L(x, D) u-\widetilde{f} e^{\tau \varphi}=0 \quad \text { in } \Omega,\left.\quad u\right|_{\Gamma_{0}}=0
$$


and

$$
\left\|u e^{-\tau \varphi}\right\|_{H^{1, \tau}(\Omega)}^{2}+\left\|u e^{-\tau \varphi}\right\|_{L^{2}(\widetilde{\Gamma})}^{2} \leq C_{27}|\tau|\|\widetilde{f}\|_{L^{2}(\Omega)}^{2}
$$

Since $\|\widetilde{f}\|_{L^{2}(\Omega)} \leq C_{28}\left(\|f\|_{L^{2}(\Omega)}+\|g\|_{H^{1 / 2}\left(\Gamma_{0}\right)}\right)$, inequality (2.56) implies (2.48).

In order to prove (2.49), we consider the extremal problem

$$
\begin{aligned}
\widetilde{J}_{\epsilon}(u)= & \frac{1}{2}\left\|u e^{-\tau \varphi}\right\|_{L^{2}(\Omega)}^{2}+\frac{1}{2 \epsilon}\left\|L(x, D) u-f e^{\tau \varphi}\right\|_{L^{2}(\Omega)}^{2} \\
& +\frac{1}{2|\tau|}\left\|u e^{-\tau \varphi}\right\|_{L_{d}^{2}(\widetilde{\Gamma})}^{2} \rightarrow \inf
\end{aligned}
$$

where

$$
u \in \widetilde{\mathcal{X}}=\left\{w \in H^{1 / 2}(\Omega)\left|L(x, D) w \in L^{2}(\Omega), w\right|_{\Gamma_{0}}=0\right\} .
$$

Here we recall that $d(x)$ is a smooth positive function on $\widetilde{\Gamma}$ which blows up like $\frac{1}{|x-y|^{8}}$ for any $y \in \partial \widetilde{\Gamma}$. There exists a unique solution to problem (2.57), (2.58), which we denote by $\widehat{u}_{\epsilon}$. By Fermat's theorem, we have

$$
\widetilde{J}_{\epsilon}^{\prime}\left(\widehat{u}_{\epsilon}\right)[\delta]=0 \quad \forall \delta \in \widetilde{\mathcal{X}} .
$$

This equality implies

$$
L(x, D)^{*} p_{\epsilon}+\widehat{u}_{\epsilon} e^{-2 \tau \varphi}=0 \quad \text { in } \Omega,\left.\quad \widehat{p}_{\epsilon}\right|_{\partial \Omega}=0,\left.\quad \frac{\partial p_{\epsilon}}{\partial \nu}\right|_{\widetilde{\Gamma}}=d \frac{\widehat{u}_{\epsilon}}{|\tau|} e^{-2 \tau \varphi} .
$$

By Proposition 2.5, we obtain

$$
\begin{array}{r}
\frac{1}{|\tau|}\left\|p_{\epsilon} e^{\tau \varphi}\right\|_{H^{1, \tau}(\Omega)}^{2}+\left\|\frac{\partial p_{\epsilon}}{\partial \nu} e^{\tau \varphi}\right\|_{L^{2}\left(\Gamma_{0}\right)}^{2}+\tau^{2}\left\|\left|\frac{\partial \Phi}{\partial z}\right| p_{\epsilon} e^{\tau \varphi}\right\|_{L^{2}(\Omega)}^{2} \\
\leq C_{29}\left(\left\|\widehat{u}_{\epsilon} e^{-\tau \varphi}\right\|_{L^{2}(\Omega)}^{2}+\frac{1}{|\tau|} \int_{\widetilde{\Gamma}^{*}}\left|\widehat{u}_{\epsilon}\right|^{2} e^{-2 \tau \varphi} d \sigma\right) \leq C_{30} \widetilde{J}_{\epsilon}\left(\widehat{u}_{\epsilon}\right) .
\end{array}
$$

Taking the scalar product of the equation in (2.59) with $\widehat{u}_{\epsilon}$, we obtain

$$
2 \widetilde{J}_{\epsilon}\left(\widehat{u}_{\epsilon}\right)+\operatorname{Re}\left(p_{\epsilon}, f e^{\tau \varphi}\right)_{L^{2}(\Omega)}=0 .
$$

Applying estimate (2.60) to this equality, we have

$$
|\tau|^{2} \widetilde{J}_{\epsilon}\left(\widehat{u}_{\epsilon}\right) \leq C_{31}\|f\|_{L^{2}(\Omega)}^{2} .
$$

Using this estimate we pass to the limit in (2.59). We conclude that

$$
\begin{aligned}
& L(x, D)^{*} p+u e^{-2 \tau \varphi}=0 \quad \text { in } \Omega,\left.\quad p\right|_{\partial \Omega}=0,\left.\quad \frac{\partial p}{\partial \nu}\right|_{\widetilde{\Gamma}}=\frac{u}{|\tau|} e^{-2 \tau \varphi}, \\
& L(x, D) u-f e^{\tau \varphi}=0 \quad \text { in } \Omega,\left.\quad u\right|_{\Gamma_{0}}=0 .
\end{aligned}
$$


Moreover (2.61) implies

$$
|\tau|^{2}\left\|u e^{-\tau \varphi}\right\|_{L^{2}(\Omega)}^{2}+|\tau|\left\|u e^{-\tau \varphi}\right\|_{L^{2}(\widetilde{\Gamma})}^{2} \leq C_{32}\|f\|_{L^{2}(\Omega)}^{2} .
$$

\section{$\S 3$. Estimates and asymptotics}

In this section we prove some estimates and obtain asymptotic expansions needed in the construction of the complex geometrical optics solutions in Section 4.

We recall that $\zeta=\xi_{1}+i \xi_{2}$ with $\xi_{1}, \xi_{2} \in \mathbb{R}$ is identified with $\xi=\left(\xi_{1}, \xi_{2}\right) \in \mathbb{R}^{2}$. By $\widetilde{\nu}=\left(\widetilde{\nu}_{1}, \widetilde{\nu}_{2}\right)$ we denote the outward unit normal vector to $S(x, \delta)$.

Consider the operator

$$
\begin{aligned}
L_{1}(x, D) & =4 \frac{\partial}{\partial z} \frac{\partial}{\partial \bar{z}}+2 A_{1} \frac{\partial}{\partial z}+2 B_{1} \frac{\partial}{\partial \bar{z}}+q_{1} \\
& =\left(2 \frac{\partial}{\partial z}+B_{1}\right)\left(2 \frac{\partial}{\partial \bar{z}}+A_{1}\right)+q_{1}-2 \frac{\partial A_{1}}{\partial z}-A_{1} B_{1} \\
& =\left(2 \frac{\partial}{\partial \bar{z}}+A_{1}\right)\left(2 \frac{\partial}{\partial z}+B_{1}\right)+q_{1}-2 \frac{\partial B_{1}}{\partial \bar{z}}-A_{1} B_{1}
\end{aligned}
$$

Let $\mathcal{A}_{1}, \mathcal{B}_{1} \in C^{6+\alpha}(\bar{\Omega})$ with some $\alpha \in(0,1)$ satisfy

$$
2 \frac{\partial \mathcal{A}_{1}}{\partial \bar{z}}=-A_{1} \text { in } \Omega,\left.\operatorname{Im} \mathcal{A}_{1}\right|_{\Gamma_{0}}=0, \quad 2 \frac{\partial \mathcal{B}_{1}}{\partial z}=-B_{1} \text { in } \Omega,\left.\operatorname{Im} \mathcal{B}_{1}\right|_{\Gamma_{0}}=0
$$

Observe that

$$
\left(2 \frac{\partial}{\partial \bar{z}}+A_{1}\right) e^{\mathcal{A}_{1}}=0 \quad \text { in } \Omega, \quad\left(2 \frac{\partial}{\partial z}+B_{1}\right) e^{\mathcal{B}_{1}}=0 \quad \text { in } \Omega .
$$

Therefore if $a(z), \Phi(z)$ are holomorphic functions and $b(\bar{z})$ is an antiholomorphic function, then

$$
\begin{aligned}
L_{1}(x, D)\left(e^{\mathcal{A}_{1}} a e^{\tau \Phi}\right) & =\left(q_{1}-2 \frac{\partial A_{1}}{\partial z}-A_{1} B_{1}\right) e^{\mathcal{A}_{1}} a e^{\tau \Phi} \\
L_{1}(x, D)\left(e^{\mathcal{B}_{1}} b e^{\tau \bar{\Phi}}\right) & =\left(q_{1}-2 \frac{\partial B_{1}}{\partial \bar{z}}-A_{1} B_{1}\right) e^{\mathcal{B}_{1}} b e^{\tau \bar{\Phi}}
\end{aligned}
$$

Let us introduce the operators

$$
\begin{aligned}
& \partial_{\bar{z}}^{-1} g=\frac{1}{2 \pi i} \int_{\Omega} \frac{g\left(\xi_{1}, \xi_{2}\right)}{\zeta-z} d \zeta \wedge d \bar{\zeta}=-\frac{1}{\pi} \int_{\Omega} \frac{g\left(\xi_{1}, \xi_{2}\right)}{\zeta-z} d \xi_{1} d \xi_{2}, \\
& \partial_{z}^{-1} g=-\frac{1}{2 \pi i} \overline{\int_{\Omega} \frac{\bar{g}\left(\xi_{1}, \xi_{2}\right)}{\zeta-z} d \zeta \wedge d \bar{\zeta}}=-\frac{1}{\pi} \int_{\Omega} \frac{g\left(\xi_{1}, \xi_{2}\right)}{\bar{\zeta}-\bar{z}} d \xi_{1} d \xi_{2}
\end{aligned}
$$

We have (see, e.g., [29, pp. 47, 56, 72]): 
Proposition 3.1. (A) Let $m \geq 0$ be an integer and $\alpha \in(0,1)$. Then $\partial_{\bar{z}}^{-1}, \partial_{z}^{-1} \in$ $\mathcal{L}\left(C^{m+\alpha}(\bar{\Omega}), C^{m+\alpha+1}(\bar{\Omega})\right)$.

(B) Let $1 \leq p \leq 2$ and $1<\gamma<\frac{2 p}{2-p}$. Then $\partial_{\bar{z}}^{-1}, \partial_{z}^{-1} \in \mathcal{L}\left(L^{p}(\Omega), L^{\gamma}(\Omega)\right)$.

(C) Let $1<p<\infty$. Then $\partial_{\bar{z}}^{-1}, \partial_{z}^{-1} \in \mathcal{L}\left(L^{p}(\Omega), W_{p}^{1}(\Omega)\right)$.

Assume that $\mathcal{A}, \mathcal{B}, A, B$ satisfy (2.12). Setting $T_{B} g=e^{\mathcal{B}} \partial_{z}^{-1}\left(e^{-\mathcal{B}} g\right)$ and $P_{A} g=$ $e^{\mathcal{A}} \partial_{\bar{z}}^{-1}\left(e^{-\mathcal{A}} g\right)$ for any $g \in C^{\alpha}(\bar{\Omega})$, we have

$$
\left(2 \frac{\partial}{\partial z}+B\right) T_{B} g=g \quad \text { in } \Omega, \quad\left(2 \frac{\partial}{\partial \bar{z}}+A\right) P_{A} g=g \quad \text { in } \Omega .
$$

We define two more operators:

$$
\begin{aligned}
& \mathcal{R}_{\tau, A} g=\frac{1}{2} e^{\mathcal{A}} e^{\tau(\bar{\Phi}-\Phi)} \partial_{\bar{z}}^{-1}\left(g e^{-\mathcal{A}} e^{\tau(\Phi-\bar{\Phi})}\right), \\
& \widetilde{\mathcal{R}}_{\tau, B} g=\frac{1}{2} e^{\mathcal{B}} e^{\tau(\bar{\Phi}-\Phi)} \partial_{z}^{-1}\left(g e^{-\mathcal{B}} e^{\tau(\Phi-\bar{\Phi})}\right)
\end{aligned}
$$

for $\mathcal{A}, \mathcal{B}, A, B$ satisfying (2.12).

The following proposition follows from straightforward calculations.

Proposition 3.2. Let $g \in C^{\alpha}(\bar{\Omega})$ for some positive $\alpha$. The function $\mathcal{R}_{\tau, A} g$ is a solution to

$$
2 \frac{\partial}{\partial \bar{z}} \mathcal{R}_{\tau, A} g-2 \tau \frac{\partial \bar{\Phi}}{\partial \bar{z}} \mathcal{R}_{\tau, A} g+A \mathcal{R}_{\tau, A} g=g \quad \text { in } \Omega
$$

The function $\widetilde{\mathcal{R}}_{\tau, B} g$ solves

$$
2 \frac{\partial}{\partial z} \widetilde{\mathcal{R}}_{\tau, B} g+2 \tau \frac{\partial \Phi}{\partial z} \widetilde{\mathcal{R}}_{\tau, B} g+B \widetilde{\mathcal{R}}_{\tau, B} g=g \quad \text { in } \Omega .
$$

Using the stationary phase argument (e.g., Bleistein and Handelsman [4]), we show

Proposition 3.3. Let $g \in L^{1}(\Omega)$ and let a function $\Phi$ satisfy (2.1) and (2.2). Then

$$
\lim _{|\tau| \rightarrow+\infty} \int_{\Omega} g e^{\tau(\Phi(z)-\overline{\Phi(z)})} d x=0
$$

For the proof, see Proposition 2.4 in [15].

Moreover we have

Proposition 3.4. Let $g \in C^{2}(\Omega),\left.g\right|_{\mathcal{O}_{\epsilon}}=0$ and $\left.g\right|_{\mathcal{H}}=0$. Then for any $1 \leq$ $p<\infty$,

$$
\left\|\mathcal{R}_{\tau, A} g+\frac{g}{2 \tau \overline{\partial_{z} \Phi}}\right\|_{L^{p}(\Omega)}+\left\|\widetilde{\mathcal{R}}_{\tau, B} g-\frac{g}{2 \tau \partial_{z} \Phi}\right\|_{L^{p}(\Omega)}=o(1 / \tau) \quad \text { as }|\tau| \rightarrow+\infty .
$$


Proof. We give a proof of the asymptotic formula for $\widetilde{\mathcal{R}}_{\tau, B} g$. The proof for $\mathcal{R}_{\tau, A} g$ is similar. Let $\widetilde{g}(\zeta, \bar{\zeta})=g e^{-\mathcal{B}}$. Then

$$
\begin{aligned}
2 e^{-\mathcal{B}} \mathcal{R}_{\tau, B} g & =-\frac{e^{\tau(\bar{\Phi}-\Phi)}}{\pi} \int_{\Omega} \frac{\widetilde{g}(\zeta, \bar{\zeta})}{\bar{\zeta}-\bar{z}} e^{\tau(\Phi(\zeta)-\overline{\Phi(\zeta)})} d \xi_{1} d \xi_{2} \\
& =-\frac{e^{\tau(\bar{\Phi}-\Phi)}}{\pi} \lim _{\delta \rightarrow+0} \int_{\Omega \backslash B(z, \delta)} \frac{\widetilde{g}(\zeta, \bar{\zeta})}{\bar{\zeta}-\bar{z}} e^{\tau(\Phi(\zeta)-\overline{\Phi(\zeta))}} d \xi_{1} d \xi_{2}
\end{aligned}
$$

Let $z=x_{1}+i x_{2}$ and suppose $x=\left(x_{1}, x_{2}\right)$ is not a critical point of $\Phi$. Then

$$
\begin{aligned}
2 e^{-\mathcal{B}} \mathcal{R}_{\tau, B} g=-\frac{e^{\tau(\bar{\Phi}-\Phi)}}{\pi \tau} \lim _{\delta \rightarrow+0} \int_{\Omega \backslash B(x, \delta)} \frac{\widetilde{g}(\zeta, \bar{\zeta})}{\bar{\zeta}-\bar{z}} \frac{\partial_{\zeta} e^{\tau(\Phi(\zeta)-\bar{\Phi}(\zeta))}}{\partial_{\zeta} \Phi(\zeta)} d \xi_{1} d \xi_{2} \\
=\frac{e^{\tau(\bar{\Phi}-\Phi)}}{\pi \tau} \lim _{\delta \rightarrow+0} \int_{\Omega \backslash B(x, \delta)} \frac{1}{\bar{\zeta}-\bar{z}} \frac{\partial}{\partial \zeta}\left(\frac{\widetilde{g}(\zeta, \bar{\zeta})}{\partial_{\zeta} \Phi(\zeta)}\right) e^{\tau(\Phi(\zeta)-\overline{\Phi(\zeta)})} d \xi_{1} d \xi_{2} \\
\quad-\frac{e^{\tau(\bar{\Phi}-\Phi)}}{\pi \tau} \lim _{\delta \rightarrow+0} \int_{S(x, \delta)} \frac{\widetilde{g}(\zeta, \bar{\zeta})}{\bar{\zeta}-\bar{z}} \frac{\widetilde{\nu}_{1}-i \widetilde{\nu}_{2}}{2 \partial_{\zeta} \Phi(\zeta)} e^{\tau(\Phi(\zeta)-\overline{\Phi(\zeta))}} d \xi_{1} d \xi_{2}
\end{aligned}
$$

Since $\left.\widetilde{g}\right|_{\mathcal{H}}=0$, we have

$$
\left|\frac{\partial}{\partial \zeta}\left(\frac{\widetilde{g}(\zeta, \bar{\zeta})}{\partial_{\zeta} \Phi(\zeta)}\right)\right| \leq C \sum_{k=1}^{\ell} \frac{\|\widetilde{g}\|_{C^{1}(\bar{\Omega})}}{\left|\xi-\widetilde{x}_{k}\right|} \in L^{p}(\Omega) \quad \forall p \in(1,2) .
$$

Hence, passing to the limit in (3.7), we obtain

$$
2 e^{-\mathcal{B}} \mathcal{R}_{\tau, B} g=\frac{e^{\tau(\bar{\Phi}-\Phi)}}{\pi \tau} \int_{\Omega} \frac{1}{\bar{\zeta}-\bar{z}} \frac{\partial}{\partial \zeta}\left(\frac{\widetilde{g}(\zeta, \bar{\zeta})}{\partial_{\zeta} \Phi(\zeta)}\right) e^{\tau(\Phi(\zeta)-\overline{\Phi(\zeta)})} d \xi_{1} d \xi_{2}-\frac{\widetilde{g}(z, \bar{z})}{\tau \partial_{z} \Phi(z)}
$$

Denote

$$
G_{\tau}(x)=\int_{\Omega} \frac{1}{\bar{\zeta}-\bar{z}} \frac{\partial}{\partial \zeta}\left(\frac{\widetilde{g}(\zeta, \bar{\zeta})}{\partial_{\zeta} \Phi(\zeta)}\right) e^{\tau(\Phi(\zeta)-\Phi(\bar{\zeta}))} d \xi_{1} d \xi_{2} .
$$

By Proposition 3.3, we see that

$$
G_{\tau}(x) \rightarrow 0 \quad \text { as }|\tau| \rightarrow+\infty \quad \forall x \in \bar{\Omega} .
$$

Denote

$$
T\left(\xi_{1}, \xi_{2}\right)=\left|\frac{\partial_{\bar{\zeta}} \widetilde{g}(\zeta, \bar{\zeta})}{\partial_{\zeta} \Phi(\zeta)}\right|_{\chi_{\Omega}\left(\xi_{1}, \xi_{2}\right)}
$$

where $\chi_{\Omega}$ is the characteristic function of $\Omega$. Clearly

$$
\left|G_{\tau}(x)\right| \leq \int_{\Omega} \frac{\left|T\left(\xi_{1}, \xi_{2}\right)\right|}{|z-\zeta|} d \xi_{1} d \xi_{2} \quad \text { a.e. in } \Omega \quad \forall \tau .
$$

By (3.8), $T \in L^{p}\left(\mathbb{R}^{2}\right)$ for any $p \in(1,2)$. For any $f \in L^{p}\left(\mathbb{R}^{2}\right)$, we set

$$
I_{r} f(z)=\int_{\mathbb{R}^{2}}|z-\zeta|^{-2 / r} f(\zeta, \bar{\zeta}) d \xi_{1} d \xi_{2} .
$$


Then, by the Hardy-Littlewood-Sobolev inequality, if $r>1$ and $\frac{1}{r}=1-\left(\frac{1}{p}-\frac{1}{q}\right)$ for $1<p<q<\infty$, then

$$
\left\|I_{r} f\right\|_{L^{q}\left(\mathbb{R}^{2}\right)} \leq C_{p, q}\|f\|_{L^{p}\left(\mathbb{R}^{2}\right)} .
$$

Set $r=2$. Then we have to choose $1 / p-1 / q=1 / 2$, that is, we can arbitrarily choose $p>2$ close to 2 , so that $q$ is arbitrarily large. Hence $\int_{\Omega} \frac{T}{|z-\zeta|} d \xi_{1} d \xi_{2}$ belongs to $L^{q}(\Omega)$ with $q>1$. By (3.9), (3.10) and the dominated convergence theorem, we obtain

$$
G_{\tau} \rightarrow 0 \quad \text { in } L^{q}(\Omega) \quad \forall q \in(1, \infty) .
$$

We now consider the contribution from the critical points.

Proposition 3.5. Let $\Phi$ satisfy (2.1) and (2.2). Let $g \in C^{4+\alpha}(\bar{\Omega})$ for some $\alpha>0$, $\left.g\right|_{\mathcal{O}_{\epsilon}}=0$ and $\left.g\right|_{\mathcal{H}}=0$. Then there exist constants $p_{k}$ such that

$$
\int_{\Omega} g e^{\tau(\Phi(z)-\overline{\Phi(z)})} d x=\frac{1}{\tau^{2}} \sum_{k=1}^{\ell} p_{k} e^{2 \tau i \psi\left(\widetilde{x}_{k}\right)}+o\left(1 / \tau^{2}\right) \quad \text { as }|\tau| \rightarrow+\infty .
$$

Proof. Let $\delta>0$ be a sufficiently small number and $\widetilde{e}_{k} \in C_{0}^{\infty}\left(B\left(\widetilde{x}_{k}, \delta\right)\right)$ with $\left.\widetilde{e}_{k}\right|_{B\left(\widetilde{x}_{k}, \delta / 2\right)} \equiv 1$. The stationary phase argument yields

$$
\begin{aligned}
I(\tau) & =\int_{\Omega} g e^{\tau(\Phi-\bar{\Phi})} d x=\sum_{k=1}^{\ell} \int_{B\left(\widetilde{x}_{k}, \delta\right)} \widetilde{e}_{k} g e^{\tau(\Phi-\bar{\Phi})} d x+o\left(1 / \tau^{2}\right) \\
& =\sum_{k=1}^{\ell} e^{2 i \tau \psi\left(\widetilde{x}_{k}\right)} \int_{B\left(\widetilde{x}_{k}, \delta\right)} \widetilde{e}_{k} g e^{\tau(\Phi-\bar{\Phi})-2 i \tau \psi\left(\widetilde{x}_{k}\right)} d x+o\left(1 / \tau^{2}\right) \quad \text { as }|\tau| \rightarrow+\infty .
\end{aligned}
$$

Since all the critical points of $\Phi$ are nondegenerate, in some neighborhood of $\widetilde{x}_{k}$ one can take local coordinates such that $\Phi-\bar{\Phi}-2 i \tau \psi\left(\widetilde{x}_{k}\right)=z^{2}-\bar{z}^{2}$. Therefore

$$
I(\tau)=\sum_{k=1}^{\ell} e^{2 i \tau \psi\left(\widetilde{x}_{k}\right)} \int_{B\left(0, \delta^{\prime}\right)} q_{k} e^{\tau\left(z^{2}-\bar{z}^{2}\right)} d x+o\left(1 / \tau^{2}\right) \quad \text { as }|\tau| \rightarrow+\infty,
$$

where $q_{k} \in C_{0}^{4}\left(B\left(0, \delta^{\prime}\right)\right)$ and $q_{k}(0)=0$. Hence there exist functions $r_{1, k}, r_{2, k} \in$ $C_{0}^{3}\left(B\left(0, \delta^{\prime}\right)\right)$ such that $q_{k}=2 z r_{1, k}+2 \bar{z} r_{2, k}$. Integrating by parts, one can decompose $I(\tau)$ as

$$
\begin{aligned}
I(\tau)= & -\frac{1}{\tau} \sum_{k=1}^{\ell} e^{2 i \tau \psi\left(\widetilde{x}_{k}\right)} \int_{B\left(0, \delta^{\prime}\right)}\left(\frac{\partial r_{1, k}}{\partial z}-\frac{\partial r_{2, k}}{\partial \bar{z}}\right) e^{\tau\left(z^{2}-\bar{z}^{2}\right)} d x+o\left(1 / \tau^{2}\right) \\
= & -\frac{1}{\tau} \sum_{k=1}^{\ell} e^{2 i \tau \psi\left(\widetilde{x}_{k}\right)} \int_{B\left(0, \delta^{\prime}\right)}\left(\frac{\partial r_{1, k}}{\partial z}-\frac{\partial r_{2, k}}{\partial \bar{z}}\right)(0) \chi(x) e^{\tau\left(z^{2}-\bar{z}^{2}\right)} d x \\
& -\frac{1}{\tau} \sum_{k=1}^{\ell} e^{2 i \tau \psi\left(\widetilde{x}_{k}\right)} \int_{B\left(0, \delta^{\prime}\right)} \widetilde{q}_{k} e^{\tau\left(z^{2}-\bar{z}^{2}\right)} d x+o\left(1 / \tau^{2}\right) \quad \text { as }|\tau| \rightarrow+\infty,
\end{aligned}
$$


where $\chi, \widetilde{q}_{k} \in C_{0}^{2}\left(B\left(0, \delta^{\prime}\right)\right),\left.\chi\right|_{B\left(0, \delta^{\prime} / 2\right)} \equiv 1$ and $\widetilde{q}_{k}(0)=0$. Hence there exist functions $\widetilde{r}_{1, k}, \widetilde{r}_{2, k} \in C_{0}^{1}\left(B\left(0, \delta^{\prime}\right)\right)$ such that $\widetilde{q}_{k}=2 z \widetilde{r}_{1, k}+2 \bar{z} \widetilde{r}_{2, k}$. Integrating by parts and applying Proposition 3.3, we obtain

$$
\begin{aligned}
\lim _{|\tau| \rightarrow+\infty} \tau \int_{B\left(0, \delta^{\prime}\right)} \widetilde{q}_{k} e^{\tau\left(z^{2}-\bar{z}^{2}\right)} d x \\
=-\sum_{k=1}^{\ell} e^{2 i \tau \psi\left(\widetilde{x}_{k}\right)} \lim _{|\tau| \rightarrow+\infty} \int_{B\left(0, \delta^{\prime}\right)}\left(\frac{\partial \widetilde{r}_{1, k}}{\partial z}-\frac{\partial \widetilde{r}_{2, k}}{\partial \bar{z}}\right) e^{\tau\left(z^{2}-\bar{z}^{2}\right)} d x=0 .
\end{aligned}
$$

Therefore (3.11) follows from a standard application of the stationary phase.

Proposition 3.6. Let $0<\epsilon^{\prime}<\epsilon$, and let a function $\Phi$ satisfy (2.1), (2.2) and $\overline{\mathcal{O}}_{\epsilon} \cap\left(\mathcal{H} \backslash \Gamma_{0}\right)=\emptyset$. Suppose that $g \in C^{\alpha}(\bar{\Omega}) \cap H^{1}(\Omega)$ for some $\alpha \in(0,1),\left.g\right|_{\mathcal{O}_{\epsilon}}=0$ and $\left.g\right|_{\mathcal{H}}=0$. Then

$$
|\tau|\left\|\widetilde{\mathcal{R}}_{\tau, B} g\right\|_{L^{\infty}\left(\mathcal{O}_{\epsilon^{\prime}}\right)}+\left\|\nabla \widetilde{\mathcal{R}}_{\tau, B} g\right\|_{L^{\infty}\left(\mathcal{O}_{\epsilon^{\prime}}\right)} \leq C_{1}\left(\epsilon^{\prime}, \alpha\right)\|g\|_{C^{\alpha}(\bar{\Omega}) \cap H^{1}(\Omega)} .
$$

Moreover

$$
\begin{aligned}
\left\|\nabla \widetilde{\mathcal{R}}_{\tau, B} g\right\|_{L^{2}(\Omega)}+|\tau|^{1 / 2}\left\|\widetilde{\mathcal{R}}_{\tau, B} g\right\|_{L^{2}(\Omega)} & +|\tau|\left\|\frac{\partial \Phi}{\partial z} \widetilde{\mathcal{R}}_{\tau, B} g\right\|_{L^{2}(\Omega)} \\
& \leq C_{2}\left(\epsilon^{\prime}, \alpha\right)\|g\|_{C^{\alpha}(\bar{\Omega}) \cap H^{1}(\Omega)} .
\end{aligned}
$$

Proof. Denote $\widetilde{g}=g e^{-\mathcal{B}}$. Let $x=\left(x_{1}, x_{2}\right)$ be an arbitrary point from $\mathcal{O}_{\epsilon^{\prime}}$ and $z=x_{1}+i x_{2}$. Then

$$
-\pi \partial_{z}^{-1}\left(e^{\tau(\Phi-\bar{\Phi})} \widetilde{g}\right)=\int_{\Omega} \frac{\widetilde{g} e^{\tau(\Phi-\bar{\Phi})}}{\bar{\zeta}-\bar{z}} d \xi_{1} d \xi_{2}=\lim _{\delta \rightarrow+0} \sum_{k=1}^{\ell} \int_{\Omega \backslash B\left(\widetilde{x}_{k}, \delta\right)} \frac{\widetilde{g} e^{\tau(\Phi-\bar{\Phi})}}{\bar{\zeta}-\bar{z}} d \xi_{1} d \xi_{2} .
$$

Integrating by parts and taking $\delta$ sufficiently small, we have

$$
\begin{aligned}
-\pi \partial_{z}^{-1}\left(e^{\tau(\Phi-\bar{\Phi})} \widetilde{g}\right) & =-\frac{1}{\tau} \lim _{\delta \rightarrow+0} \int_{\Omega \backslash \bigcup_{k=1}^{e} B\left(\widetilde{x}_{k}, \delta\right)} \frac{\frac{\partial \widetilde{g}}{\partial \zeta}}{(\bar{\zeta}-\bar{z}) \frac{\partial \Phi}{\partial \zeta}} e^{\tau(\Phi-\bar{\Phi})} d \xi_{1} d \xi_{2} \\
+ & \frac{1}{\tau} \lim _{\delta \rightarrow+0} \int_{\Omega \backslash \bigcup_{k=1}^{e} B\left(\widetilde{x}_{k}, \delta\right)} \frac{\widetilde{g} \frac{\frac{\partial}{}^{2} \Phi}{\partial \zeta^{2}}}{(\bar{\zeta}-\bar{z})\left(\frac{\partial \Phi}{\partial \zeta}\right)^{2}} e^{\tau(\Phi-\bar{\Phi})} d \xi_{1} d \xi_{2} \\
+ & \frac{1}{2 \tau} \lim _{\delta \rightarrow+0} \int_{\bigcup_{k=1}^{e} S\left(\widetilde{x}_{k}, \delta\right)}\left(\widetilde{\nu}_{1}-i \widetilde{\nu}_{2}\right) \frac{\widetilde{g}}{(\bar{\zeta}-\bar{z}) \frac{\partial \Phi}{\partial \zeta}} e^{\tau(\Phi-\bar{\Phi})} d \sigma .
\end{aligned}
$$

Since $\left.g\right|_{\mathcal{H}}=0$, we have $\|g\|_{C^{0}\left(S\left(\widetilde{x}_{k}, \delta\right)\right)} \leq \delta^{\alpha}\|g\|_{C^{\alpha}(\bar{\Omega})}$. Using this inequality and the fact that all the critical points of $\Phi$ are nondegenerate, we obtain

$$
\frac{1}{2 \tau} \lim _{\delta \rightarrow+0} \int_{\bigcup_{k=1}^{\ell} S\left(\widetilde{x}_{k}, \delta\right)}\left(\widetilde{\nu}_{1}-i \widetilde{\nu}_{2}\right) \frac{\widetilde{g}}{(\bar{\zeta}-\bar{z}) \frac{\partial \Phi}{\partial \zeta}} e^{\tau(\Phi-\bar{\Phi})} d \sigma=0
$$


Since $\left|\frac{\widetilde{g} \frac{\partial^{2} \Phi}{\partial \zeta^{2}}}{\left(\frac{\partial \Phi}{\partial \zeta}\right)^{2}}(\zeta, \bar{\zeta})\right| \leq C_{3}\|\widetilde{g}\|_{C^{\alpha}(\bar{\Omega})} \sum_{k=1}^{\ell} \frac{1}{\left|\xi-\widetilde{x}_{k}\right|^{2-\alpha}}$, we see that $\frac{\widetilde{g} \frac{\partial^{2} \Phi}{\partial \zeta^{2}}}{\left(\frac{\partial \Phi}{\partial \zeta}\right)^{2}}(\zeta, \bar{\zeta}) \in L^{1}(\Omega)$ and

$$
\begin{aligned}
-\pi \partial_{z}^{-1}\left(e^{\tau(\Phi-\bar{\Phi})} \widetilde{g}\right)= & -\frac{1}{\tau} \int_{\Omega} \frac{\frac{\partial \widetilde{g}}{\partial \zeta}}{(\bar{\zeta}-\bar{z}) \frac{\partial \Phi}{\partial \zeta}} e^{\tau(\Phi-\bar{\Phi})} d \xi_{1} d \xi_{2} \\
& +\frac{1}{\tau} \int_{\Omega} \frac{\widetilde{g} \frac{\partial^{2} \Phi}{\partial \zeta^{2}}}{(\bar{\zeta}-\bar{z})\left(\frac{\partial \Phi}{\partial \zeta}\right)^{2}} e^{\tau(\Phi-\bar{\Phi})} d \xi_{1} d \xi_{2}
\end{aligned}
$$

From this equality, Proposition 3.3 and the definition (3.3) of the operator $\widetilde{\mathcal{R}}_{\tau, B}$, the estimate (3.12) follows immediately. In order to prove (3.13), we observe

$$
\begin{aligned}
\frac{\partial \widetilde{\mathcal{R}}_{\tau, B} g}{\partial \bar{z}}= & \frac{\partial \mathcal{B}}{\partial \bar{z}} \widetilde{\mathcal{R}}_{\tau, B} g+\widetilde{\mathcal{R}}_{\tau, B}\left\{\frac{\partial g}{\partial \bar{z}}-\frac{\partial \mathcal{B}}{\partial \bar{z}} g\right\} \\
& +\frac{\tau}{2 \pi} e^{\tau(\bar{\Phi}-\Phi)+\mathcal{B}} \int_{\Omega} \frac{\frac{\partial \bar{\Phi}(\zeta)}{\partial \bar{\zeta}}-\frac{\partial \bar{\Phi}(z)}{\partial \bar{z}}}{\bar{\zeta}-\bar{z}} e^{\tau(\Phi-\bar{\Phi})} d \xi_{1} d \xi_{2}
\end{aligned}
$$

Proposition 3.1 yields

$$
\left\|\frac{\partial \mathcal{B}}{\partial \bar{z}} \widetilde{\mathcal{R}}_{\tau, B} g+\widetilde{\mathcal{R}}_{\tau, B}\left\{\frac{\partial g}{\partial \bar{z}}-\frac{\partial \mathcal{B}}{\partial \bar{z}} g\right\}\right\|_{L^{2}(\Omega)} \leq C_{4}\|g\|_{H^{1}(\Omega)}
$$

Using arguments similar to (3.14) and (3.15), we obtain

$$
\left\|\frac{\tau}{2 \pi} \int_{\Omega} \frac{\frac{\partial \bar{\Phi}(\zeta)}{\partial \bar{\zeta}}-\frac{\partial \bar{\Phi}(z)}{\partial \bar{z}}}{\bar{\zeta}-\bar{z}} \widetilde{g} e^{\tau(\Phi-\bar{\Phi})} d \xi_{1} d \xi_{2}\right\|_{L^{2}(\Omega)} \leq C_{5}\|g\|_{C^{\alpha}(\bar{\Omega}) \cap H^{1}(\Omega)} .
$$

Hence

$$
\left\|\frac{\partial \widetilde{\mathcal{R}}_{\tau, B} g}{\partial \bar{z}}\right\|_{L^{2}(\Omega)} \leq C_{6}\|g\|_{C^{\alpha}(\bar{\Omega}) \cap H^{1}(\Omega)} .
$$

Combining this estimate with (3.12), we conclude that

$$
\left\|\nabla \widetilde{\mathcal{R}}_{\tau, B} g\right\|_{L^{2}(\Omega)} \leq C_{7}\|g\|_{C^{\alpha}(\bar{\Omega}) \cap H^{1}(\Omega)} .
$$

Using this estimate and equation (3.5), we have

$$
|\tau|\left\|\frac{\partial \Phi}{\partial z} \widetilde{\mathcal{R}}_{\tau, B} g\right\|_{L^{2}(\Omega)} \leq C_{8}\|g\|_{C^{\alpha}(\bar{\Omega}) \cap H^{1}(\Omega)}
$$

finishing the proof of the proposition.

Let $e_{1}, e_{2} \in C^{\infty}(\bar{\Omega})$ satisfy

$$
e_{1}+e_{2}=1 \text { in } \Omega
$$

and let $e_{2}$ vanish in some neighborhood of $\mathcal{H} \backslash \Gamma_{0}$ and $e_{1}$ vanish in a neighborhood 
of $\partial \Omega$. We set

$$
\mathcal{P}(x, D)\left(u e^{\tau \Phi}\right)=\left(2 \frac{\partial}{\partial \bar{z}}+A\right)\left(2 \frac{\partial}{\partial z}+B\right)\left(u e^{\tau \Phi}\right) .
$$

Proposition 3.7. Let $A, B \in C^{5+\alpha}(\bar{\Omega})$ and $\mathcal{A}, \mathcal{B} \in C^{6+\alpha}(\bar{\Omega})$ with some $\alpha \in(0,1)$ satisfy (2.12). Let $e_{1}, e_{2}$ be defined as in (3.16). Let $g \in L^{p}(\Omega)$ for some $p>2$, $\operatorname{supp} g \subset \subset \operatorname{supp} e_{1}$ and $\operatorname{dist}\left(\Gamma_{0}, \operatorname{supp} g\right)>0$. Define

$$
u=\widetilde{\mathcal{R}}_{\tau, B}\left(e_{1}\left(P_{A} g-\widetilde{M} e^{\mathcal{A}}\right)\right)+\frac{e_{2}\left(P_{A} g-\widetilde{M} e^{\mathcal{A}}\right)}{2 \tau \partial_{z} \Phi},
$$

where $\widetilde{M}=\widetilde{M}(z)$ is a polynomial such that $\left.\frac{\partial^{k}}{\partial z^{k}}\left(P_{A} g-\widetilde{M} e^{\mathcal{A}}\right)\right|_{\mathcal{H}}=0$ for any $k$ from $\{0, \ldots, 6\}$. Then

$$
\mathcal{P}(x, D)\left(u e^{\tau \Phi}\right)=g e^{\tau \Phi}+\frac{e^{\tau \varphi}}{|\tau|} h_{\tau} \quad \text { as }|\tau| \rightarrow+\infty
$$

where

$$
\left\|h_{\tau}\right\|_{L^{\infty}(\Omega)} \leq C_{9}(p)\|g\|_{L^{p}(\Omega)}
$$

and for some sufficiently small positive $\epsilon^{\prime}$ we have

$$
\frac{1}{|\tau|^{1 / 2}}\|\nabla u\|_{L^{2}(\Omega)}+|\tau|^{1 / 2}\|u\|_{L^{2}(\Omega)}+\|u\|_{H^{1, \tau}\left(\mathcal{O}_{\epsilon^{\prime}}\right)} \leq C_{10}\|g\|_{L^{p}(\Omega)} .
$$

Proof. By Proposition 3.1, $P_{A} g$ belongs to $W_{p}^{1}(\Omega)$. Since $p>2$, by the Sobolev embedding theorem there exists $\alpha>0$ such that $P_{A} g \in C^{\alpha}(\bar{\Omega})$. By properties of elliptic operators and the fact that $\operatorname{supp} e_{2} \cap \operatorname{supp} g=\emptyset$, we have $P_{A} g \in C^{5}\left(\operatorname{supp} e_{2}\right)$. The estimate (3.18) follows from Proposition 3.6. Short calculations give

$$
\mathcal{P}(x, D)\left(u e^{\tau \Phi}\right)=g e^{\tau \Phi}+\frac{e^{\tau \Phi}}{\tau} \mathcal{P}(x, D)\left(\frac{e_{2}\left(P_{A} g-\widetilde{M} e^{\mathcal{A}}\right)}{2 \partial_{z} \Phi}\right) .
$$

This formula implies (3.17) with $h_{\tau}=e^{i \tau \psi} \mathcal{P}(x, D)\left(\frac{e_{2}\left(P_{A} g-\widetilde{M} e^{\mathcal{A}}\right)}{2 \partial_{z} \Phi}\right) / \operatorname{sign} \tau$.

The following proposition is critical to the construction of the complex geometrical optics solutions.

Proposition 3.8. Let $f \in L^{p}(\Omega)$ for some $p>2$, $\operatorname{dist}\left(\overline{\Gamma_{0}}, \operatorname{supp} f\right)>0, q \in$ $H^{1 / 2}\left(\Gamma_{0}\right)$, and $\epsilon^{\prime}$ a small positive number such that $\overline{\mathcal{O}_{\epsilon^{\prime}}} \cap\left(\mathcal{H} \backslash \Gamma_{0}\right)=\emptyset$. Then there exists $\tau_{0}$ such that for all $|\tau|>\tau_{0}$ there exists a solution to the boundary value problem

$$
L(x, D) w=f e^{\tau \Phi} \quad \text { in } \Omega,\left.\quad w\right|_{\Gamma_{0}}=q e^{\tau \varphi} / \tau
$$

such that

$$
\begin{array}{r}
\sqrt{|\tau|}\left\|w e^{-\tau \varphi}\right\|_{L^{2}(\Omega)}+\frac{1}{\sqrt{|\tau|}}\left\|(\nabla w) e^{-\tau \varphi}\right\|_{L^{2}(\Omega)}+\left\|w e^{-\tau \varphi}\right\|_{H^{1, \tau}\left(\mathcal{O}_{\epsilon^{\prime}}\right)} \\
\leq C_{11}\left(\|f\|_{L^{p}(\Omega)}+\|q\|_{H^{1 / 2}\left(\Gamma_{0}\right)}\right)
\end{array}
$$


Proof. Let $\chi \in C_{0}^{\infty}(\Omega)$ be equal to one in some neighborhood of the set $\mathcal{H} \backslash \Gamma_{0}$. By Proposition 2.6 there exists a solution to the problem (3.20) with inhomogeneous term $(1-\chi) f$ and boundary data $q / \tau$ such that

$$
\left\|w_{1} e^{-\tau \varphi}\right\|_{H^{1, \tau}(\Omega)} \leq C_{12}\left(\|f\|_{L^{2}(\Omega)}+\|q\|_{H^{1 / 2}\left(\Gamma_{0}\right)}\right) .
$$

Denote

$$
w_{2}=\widetilde{\mathcal{R}}_{-\tau, B}\left(e_{1}\left(P_{A}(\chi f)-\widetilde{M} e^{\mathcal{A}}\right)\right)+\frac{e_{2}\left(P_{A}(\chi f)-\widetilde{M} e^{\mathcal{A}}\right)}{2 \tau \partial_{z} \Phi}
$$

where $\widetilde{M}=\widetilde{M}(z)$ is a polynomial such that $\left.\frac{\partial^{k}}{\partial z^{k}}\left(P_{A}(\chi f)-\widetilde{M} e^{\mathcal{A}}\right)\right|_{\mathcal{H}}=0$ for any $k$ from $\{0, \ldots, 6\}$. Let $q_{\tau}$ be the restriction of $w_{2}$ to $\Gamma_{0}$. By $(3.12)$ there exists a constant $C_{13}$ independent of $\tau$ such that

$$
|\tau|^{1 / 2}\left\|q_{\tau}\right\|_{H^{1 / 2}\left(\Gamma_{0}\right)} \leq C_{13}\|f\|_{L^{p}(\Omega)} .
$$

By Proposition 3.7 there exists a constant $C_{14}$ independent of $\tau$ such that

$$
\begin{array}{r}
\sqrt{|\tau|}\left\|w_{2} e^{-\tau \varphi}\right\|_{L^{2}(\Omega)}+\frac{1}{\sqrt{|\tau|}}\left\|\left(\nabla w_{2}\right) e^{-\tau \varphi}\right\|_{L^{2}(\Omega)}+\left\|w_{2} e^{-\tau \varphi}\right\|_{H^{1, \tau}\left(\mathcal{O}_{\epsilon^{\prime}}\right)} \\
\leq C_{14}\|f\|_{L^{p}(\Omega)} .
\end{array}
$$

Let $\widetilde{a}_{\tau}, \widetilde{b}_{\tau} \in H^{1}(\Omega)$ be holomorphic and antiholomorphic functions, respectively, such that $\left.\left(\widetilde{a}_{\tau} e^{\mathcal{A}}+\widetilde{b}_{\tau} e^{\mathcal{B}}\right)\right|_{\Gamma_{0}}=-q_{\tau}$. By (3.22) and Proposition 2.3, there exist constants $C_{15}, C_{16}$ independent of $\tau$ such that

$$
\left\|\widetilde{a}_{\tau}\right\|_{H^{1}(\Omega)}+\left\|\widetilde{b}_{\tau}\right\|_{H^{1}(\Omega)} \leq C_{15}\left\|q_{\tau}\right\|_{H^{1 / 2}\left(\Gamma_{0}\right)} \leq C_{16} \frac{\|f\|_{L^{p}(\Omega)}}{\sqrt{|\tau|}} .
$$

The function $W=\left(w_{2}+\widetilde{a}_{\tau} e^{\mathcal{A}}\right) e^{\tau \Phi}+\widetilde{b}_{\tau} e^{\mathcal{B}+\tau \bar{\Phi}}$ satisfies

$$
L(x, D) W=\chi f e^{\tau \Phi}+e^{\tau \varphi} \frac{\tilde{h}_{\tau}}{\sqrt{|\tau|}} \quad \text { in } \Omega,\left.\quad W\right|_{\Gamma_{0}}=0,
$$

where

$$
\left\|\widetilde{h}_{\tau}\right\|_{L^{2}(\Omega)} \leq C_{17}\|f\|_{L^{2}(\Omega)}
$$

with some constant $C_{17}$ independent of $\tau$. By (3.23) and (3.24), we have

$$
\begin{array}{r}
\sqrt{|\tau|}\left\|W e^{-\tau \varphi}\right\|_{L^{2}(\Omega)}+\frac{1}{\sqrt{|\tau|}}\left\|(\nabla W) e^{-\tau \varphi}\right\|_{L^{2}(\Omega)}+\left\|W e^{-\tau \varphi}\right\|_{H^{1, \tau}\left(\mathcal{O}_{\epsilon^{\prime}}\right)} \\
\leq C_{18}\|f\|_{L^{p}(\Omega)}
\end{array}
$$


Let $\widetilde{W}$ be a solution to problem (2.47) with inhomogeneous term $f=-\widetilde{h}_{\tau} / \sqrt{|\tau|}$ and zero boundary value $g \equiv 0$. Estimate (2.48) in Proposition 2.6 takes the form

$$
\left\|\widetilde{W} e^{-\tau \varphi}\right\|_{H^{1, \tau}(\Omega)} \leq C_{19}\left\|\widetilde{h}_{\tau}\right\|_{L^{2}(\Omega)} \leq C_{20}\|f\|_{L^{2}(\Omega)} .
$$

The function $w=w_{1}+W+\widetilde{W}$ solves (3.20). The conclusion, that is, the estimate for $w$ follows from (3.21), (3.26) and (3.27).

\section{§4. Complex geometrical optics solutions}

For a complex-valued vector field $\left(A_{1}, B_{1}\right)$ and a complex-valued potential $q_{1}$, we will construct solutions to the boundary value problem

$$
L_{1}(x, D) u_{1}=0 \quad \text { in } \Omega,\left.\quad u_{1}\right|_{\Gamma_{0}}=0,
$$

of the form

$$
u_{1}(x)=a_{\tau}(z) e^{\mathcal{A}_{1}+\tau \Phi}+d_{\tau}(\bar{z}) e^{\mathcal{B}_{1}+\tau \bar{\Phi}}+u_{11} e^{\tau \varphi}+u_{12} e^{\tau \varphi} .
$$

We recall that $L_{1}(x, D)$ is defined by $(3.1)$, and $\mathcal{A}_{1}$ and $\mathcal{B}_{1}$ are defined by (3.2) respectively for $A_{1}$ and $B_{1}$; moreover $a_{\tau}(z)=a(z)+a_{1}(z) / \tau+a_{2, \tau}(z) / \tau^{2}$, $d_{\tau}(\bar{z})=d(\bar{z})+d_{1}(\bar{z}) / \tau+d_{2, \tau}(\bar{z}) / \tau^{2}$, and

$$
\begin{gathered}
a, d \in C^{5+\alpha}(\bar{\Omega}), \quad \frac{\partial a}{\partial \bar{z}}=0 \quad \text { in } \Omega, \quad \frac{\partial d}{\partial z}=0 \quad \text { in } \Omega, \\
\left.\left(a e^{\mathcal{A}_{1}}+d e^{\mathcal{B}_{1}}\right)\right|_{\Gamma_{0}}=0 .
\end{gathered}
$$

Let $\widetilde{x}$ be some fixed point from $\mathcal{H} \backslash \partial \Omega$. Suppose in addition that

$$
\left.\frac{\partial^{k} a}{\partial z^{k}}\right|_{\mathcal{H} \backslash\{\widetilde{x}\}}=\left.\frac{\partial^{k} d}{\partial \bar{z}^{k}}\right|_{\mathcal{H} \backslash\{\widetilde{x}\}}=0 \quad \forall k \in\{0, \ldots, 5\}, \quad a(\widetilde{x}) \neq 0, d(\widetilde{x}) \neq 0 .
$$

Such functions exist by Proposition 7.2.

Denote

$$
\begin{aligned}
& g_{1}=T_{B_{1}}\left(\left(q_{1}-2 \frac{\partial B_{1}}{\partial \bar{z}}-A_{1} B_{1}\right) d e^{\mathcal{B}_{1}}\right)-M_{2}(\bar{z}) e^{\mathcal{B}_{1}}, \\
& g_{2}=P_{A_{1}}\left(\left(q_{1}-2 \frac{\partial A_{1}}{\partial z}-A_{1} B_{1}\right) a e^{\mathcal{A}_{1}}\right)-M_{1}(z) e^{\mathcal{A}_{1}},
\end{aligned}
$$

where $M_{1}(z)$ and $M_{2}(\bar{z})$ are polynomials chosen such that

$$
\left.\frac{\partial^{k} g_{1}}{\partial \bar{z}^{k}}\right|_{\mathcal{H}}=\left.\frac{\partial^{k} g_{2}}{\partial z^{k}}\right|_{\mathcal{H}}=0 \quad \forall k \in\{0, \ldots, 6\} .
$$

Thanks to our assumptions on the regularity of $A_{1}, B_{1}$ and $q_{1}$, the functions $g_{1}, g_{2}$ belong to $C^{6+\alpha}(\bar{\Omega})$. 
By (4.6) and (4.5),

$$
\left.\frac{\partial^{k+j} g_{1}}{\partial z^{k} \partial \bar{z}^{j}}\right|_{\mathcal{H} \backslash\{\widetilde{x}\}}=\left.\frac{\partial^{k+j} g_{2}}{\partial z^{k} \partial \bar{z}^{j}}\right|_{\mathcal{H} \backslash\{\widetilde{x}\}}=0 \quad \text { if } k+j \leq 6 .
$$

The function $a_{1}(z)$ is holomorphic in $\Omega$ and $d_{1}(\bar{z})$ is antiholomorphic in $\Omega$ and

$$
a_{1} e^{\mathcal{A}_{1}}+d_{1} e^{\mathcal{B}_{1}}=\frac{g_{1}}{2 \overline{\partial_{z} \Phi}}+\frac{g_{2}}{2 \partial_{z} \Phi} \quad \text { on } \Gamma_{0} .
$$

The existence of such functions is given again by Proposition 2.3. Observe that by (4.7) the functions $e_{2} g_{1} / \overline{\partial_{z} \Phi}, e_{2} g_{2} / \partial_{z} \Phi$ belong to the space $C^{4}(\bar{\Omega})$. Here we recall that $e_{2} \in C^{\infty}(\bar{\Omega})$ vanishes in some neighborhood of $\mathcal{H} \backslash \Gamma_{0}$ and that (3.16) holds. Let

$$
\begin{aligned}
& \widehat{g}_{1}=T_{B_{1}}\left(\left(q_{1}-2 \frac{\partial B_{1}}{\partial \bar{z}}-A_{1} B_{1}\right) d_{1} e^{\mathcal{B}_{1}}\right)-\widehat{M}_{2}(\bar{z}) e^{\mathcal{B}_{1}} \\
& \widehat{g}_{2}=P_{A_{1}}\left(\left(q_{1}-2 \frac{\partial A_{1}}{\partial z}-A_{1} B_{1}\right) a_{1} e^{\mathcal{A}_{1}}\right)-\widehat{M}_{1}(z) e^{\mathcal{A}_{1}}
\end{aligned}
$$

where $\widehat{M}_{1}(z)$ and $\widehat{M}_{2}(\bar{z})$ are polynomials chosen such that

$$
\left.\frac{\partial^{k} \widehat{g}_{1}}{\partial \bar{z}^{k}}\right|_{\mathcal{H}}=\left.\frac{\partial^{k} \widehat{g}_{2}}{\partial z^{k}}\right|_{\mathcal{H}}=0 \quad \forall k \in\{0, \ldots, 3\} .
$$

The function $u_{11}$ is given by (recall (3.3))

$$
\begin{aligned}
u_{11}= & -e^{-i \tau \psi} \mathcal{R}_{-\tau, A_{1}}\left\{e_{1}\left(g_{1}+\widehat{g}_{1} / \tau\right)\right\}-e^{-i \tau \psi} \frac{e_{2}\left(g_{1}+\widehat{g}_{1} / \tau\right)}{2 \tau \overline{\partial_{z} \Phi}} \\
& -e^{i \tau \psi} \widetilde{\mathcal{R}}_{\tau, B_{1}}\left\{e_{1}\left(g_{2}+\widehat{g}_{2} / \tau\right)\right\}-e^{i \tau \psi} \frac{e_{2}\left(g_{2}+\widehat{g}_{2} / \tau\right)}{2 \tau \partial_{z} \Phi}
\end{aligned}
$$

We set

$$
\Lambda_{+}(\tau, \widetilde{x})=\frac{e^{2 i \tau \psi(\widetilde{x})}}{\left|\operatorname{det} \psi^{\prime \prime}(x)\right|^{1 / 2}}, \quad \Lambda_{-}(\tau, \widetilde{x})=\frac{e^{-2 i \tau \psi(\widetilde{x})}}{\left|\operatorname{det} \psi^{\prime \prime}(x)\right|^{1 / 2}}
$$

Now let us determine the functions $u_{12}, a_{2}(z)$ and $d_{2}(\bar{z})$.

First we can obtain the following asymptotic formulae for any point on $\partial \Omega$ :

$$
\begin{aligned}
\left.\mathcal{R}_{-\tau, A_{1}}\left\{e_{1} g_{1}\right\}\right|_{\partial \Omega} & =\frac{e^{\mathcal{A}_{1}+2 i \tau \psi}}{2 \tau^{2}}\left(\frac{\Lambda_{-}(\tau, \widetilde{x}) \sigma_{1}(\widetilde{x})}{(z-\widetilde{z})^{2}}+\frac{\Lambda_{-}(\tau, \widetilde{x}) m_{1}(\widetilde{x})}{\widetilde{z}-z}\right)+\mathcal{W}_{\tau, 1}, \\
\left.\widetilde{\mathcal{R}}_{\tau, B_{1}}\left\{e_{1} g_{2}\right\}\right|_{\partial \Omega} & =\frac{e^{\mathcal{B}_{1}-2 i \tau \psi}}{2 \tau^{2}}\left(\frac{\Lambda_{+}(\tau, \widetilde{x}) \widetilde{\sigma}_{1}(\widetilde{x})}{(\bar{z}-\overline{\widetilde{z}})^{2}}+\frac{\Lambda_{+}(\tau, \widetilde{x}) \widetilde{m}_{1}(\widetilde{x})}{\overline{\widetilde{z}}-\bar{z}}\right)+\mathcal{W}_{\tau, 2}
\end{aligned}
$$

where

$$
\sigma_{1}(\widetilde{x})=\frac{\partial_{z} \widetilde{g}_{1}(\widetilde{x})}{\partial_{z}^{2} \Phi(\widetilde{x})}, \quad m_{1}(\widetilde{x})=\frac{1}{2}\left(\frac{\partial_{z} \widetilde{g}_{1}(\widetilde{x})}{\partial_{z}^{2} \Phi(\widetilde{x})} \frac{\partial_{z}^{3} \Phi(\widetilde{x})}{\partial_{z}^{2} \Phi(\widetilde{x})}+\frac{\partial_{\bar{z}}^{2} \widetilde{g}_{1}(\widetilde{x})}{\partial_{z}^{2} \Phi(\widetilde{x})}-\frac{\partial_{z}^{2} \widetilde{g}_{1}(\widetilde{x})}{\partial_{z}^{2} \Phi(\widetilde{x})}\right)
$$




$$
\widetilde{\sigma}_{1}(\widetilde{x})=\frac{\partial_{\bar{z}} \widetilde{g}_{2}(\widetilde{x})}{\partial_{z}^{2} \Phi(\widetilde{x})}, \quad \widetilde{m}_{1}(\widetilde{x})=\frac{1}{2}\left(\frac{\partial_{\bar{z}} \widetilde{g}_{2}(\widetilde{x})}{\overline{\partial_{z}^{2} \Phi(\widetilde{x})}} \frac{\partial_{z}^{3} \bar{\Phi}(\widetilde{x})}{\partial_{\bar{z}}^{2} \bar{\Phi}(\widetilde{x})}-\frac{\partial_{\bar{z}}^{2} \widetilde{g}_{2}(\widetilde{x})}{\overline{\partial_{z}^{2} \Phi(\widetilde{x})}}+\frac{\partial_{z}^{2} \widetilde{g}_{2}(\widetilde{x})}{\partial_{z}^{2} \Phi(\widetilde{x})}\right),
$$

$\widetilde{g}_{1}=e^{-\mathcal{A}_{1}} g_{1}, \widetilde{g}_{2}=e^{-\mathcal{B}_{1}} g_{2}$, and $\mathcal{W}_{\tau, 1}, \mathcal{W}_{\tau, 2} \in H^{1 / 2}\left(\Gamma_{0}\right)$ satisfy

$$
\left\|\mathcal{W}_{\tau, 1}\right\|_{H^{1 / 2}\left(\Gamma_{0}\right)}+\left\|\mathcal{W}_{\tau, 2}\right\|_{H^{1 / 2}\left(\Gamma_{0}\right)}=o\left(1 / \tau^{2}\right) \quad \text { as }|\tau| \rightarrow+\infty .
$$

The proof of (4.10) and (4.11) is given in Section 8.

Denote

$$
p_{+}(x)=e^{\mathcal{A}_{1}(x)}\left(\frac{\sigma_{1}(\widetilde{x})}{(z-\widetilde{z})^{2}}+\frac{m_{1}(\widetilde{x})}{\widetilde{z}-z}\right), \quad p_{-}(x)=e^{\mathcal{B}_{1}(x)}\left(\frac{\widetilde{\sigma}_{1}(\widetilde{x})}{(\bar{z}-\overline{\widetilde{z}})^{2}}+\frac{\widetilde{m}_{1}(\widetilde{x})}{\overline{\widetilde{z}}-\bar{z}}\right) .
$$

Thanks to Proposition 2.3 we can define $a_{2, \pm}(z) \in C^{2}(\bar{\Omega})$ and $d_{2, \pm}(\bar{z}) \in C^{2}(\bar{\Omega})$ satisfying

$$
a_{2, \pm} e^{\mathcal{A}_{1}}+d_{2, \pm} e^{\mathcal{B}_{1}}=p_{ \pm} \quad \text { on } \Gamma_{0} .
$$

Straightforward computations give

$$
\begin{aligned}
& L_{1}(x, D)\left(\left(a+a_{1} / \tau\right) e^{\mathcal{A}_{1}+\tau \Phi}+\left(d+d_{1} / \tau\right) e^{\mathcal{B}_{1}+\tau \Phi}+e^{\tau \varphi} u_{11}\right) \\
= & \left(q_{1}-2 \frac{\partial B_{1}}{\partial \bar{z}}-A_{1} B_{1}\right) e^{\tau \Phi}\left(-\widetilde{\mathcal{R}}_{\tau, B_{1}}\left\{e_{1}\left(g_{2}+\widehat{g}_{2} / \tau\right)\right\}-\frac{e_{2}\left(g_{2}+\widehat{g}_{2} / \tau\right)}{2 \tau \partial_{z} \Phi}\right) \\
& +\left(q_{1}-2 \frac{\partial A_{1}}{\partial z}-A_{1} B_{1}\right) e^{\tau \Phi}\left(-\mathcal{R}_{-\tau, A_{1}}\left\{e_{1}\left(g_{1}+\widehat{g}_{1} / \tau\right)\right\}-\frac{e_{2}\left(g_{1}+\widehat{g}_{1} / \tau\right)}{2 \tau \bar{\partial}_{z} \Phi}\right) \\
& -\left(2 \frac{\partial}{\partial \bar{z}}+A_{1}\right)\left(2 \frac{\partial}{\partial z}+B_{1}\right) \frac{e_{2}\left(g_{2}+\widehat{g}_{2} / \tau\right) e^{\tau \Phi}}{2 \tau \partial_{z} \Phi} \\
& -\left(2 \frac{\partial}{\partial z}+B_{1}\right)\left(2 \frac{\partial}{\partial \bar{z}}+A_{1}\right) \frac{e_{2}\left(g_{1}+\widehat{g}_{1} / \tau\right) e^{\tau \Phi}}{2 \tau \overline{\partial_{z} \Phi}} .
\end{aligned}
$$

Using Proposition 3.4 we transform the right-hand side of (4.16) as follows:

$$
\begin{aligned}
& L_{1}(x, D)\left(\left(a+a_{1} / \tau\right) e^{\mathcal{A}_{1}+\tau \Phi}+\left(d+d_{1} / \tau\right) e^{\mathcal{B}_{1}+\tau \bar{\Phi}}+u_{11} e^{\tau \varphi}\right) \\
&=-\left(q_{1}-2 \frac{\partial A_{1}}{\partial z}-A_{1} B_{1}\right) e^{\tau \Phi} \frac{g_{1}}{2 \tau \partial_{z} \Phi} \\
&-\left(2 \frac{\partial}{\partial \bar{z}}+A_{1}\right)\left(2 \frac{\partial}{\partial z}+B_{1}\right) \frac{e_{2}\left(g_{2}+\widehat{g}_{2} / \tau\right) e^{\tau \Phi}}{2 \tau \partial_{z} \Phi} \\
&-\left(2 \frac{\partial}{\partial z}+B_{1}\right)\left(2 \frac{\partial}{\partial \bar{z}}+A_{1}\right) \frac{e_{2}\left(g_{1}+\widehat{g}_{1} / \tau\right) e^{\tau \Phi}}{2 \tau \overline{\partial_{z} \Phi}} \\
&-\left(q_{1}-2 \frac{\partial B_{1}}{\partial \bar{z}}-A_{1} B_{1}\right) e^{\tau \bar{\Phi}} \frac{g_{2}}{2 \tau \overline{\partial_{z} \Phi}} \\
&+e^{\tau \varphi} \chi_{\mathcal{O}_{\epsilon}} O_{L^{4}(\Omega)}\left(1 / \tau^{2}\right)+e^{\tau \varphi} \chi_{\Omega \backslash \mathcal{O}_{\epsilon^{\prime}} o_{L^{4}(\Omega)}}(1 / \tau)
\end{aligned}
$$

as $|\tau| \rightarrow+\infty$. 
We are looking for $u_{12}$ in the form $u_{12}=u_{0}+u_{-1}$. The function $u_{-1}$ is given by

$$
u_{-1}=\frac{e^{i \tau \psi}}{\tau} \widetilde{\mathcal{R}}_{\tau, B_{1}}\left\{e_{1} g_{5}\right\}+\frac{e^{-i \tau \psi}}{\tau} \mathcal{R}_{-\tau, A_{1}}\left\{e_{1} g_{6}\right\}+\frac{e_{2} g_{5} e^{i \tau \psi}}{2 \tau^{2} \partial_{z} \Phi}+\frac{e_{2} g_{6} e^{-i \tau \psi}}{2 \tau^{2} \overline{\partial_{z} \Phi}}
$$

where

$$
\begin{aligned}
g_{5}=P_{A_{1}}( & \frac{\left(q_{1}-2 \frac{\partial B_{1}}{\partial \bar{z}}-A_{1} B_{1}\right) g_{2}}{2 \partial_{z} \Phi} \\
& \left.+\left(2 \frac{\partial}{\partial \bar{z}}+A_{1}\right)\left(2 \frac{\partial}{\partial z}+B_{1}\right) \frac{e_{2}\left(g_{2}+\widehat{g}_{2} / \tau\right)}{2 \partial_{z} \Phi}\right)-M_{5}(z) e^{\mathcal{A}_{1}} \\
g_{6}=T_{B_{1}}\left(\frac{\left.\left(q_{1}-2 \frac{\partial A_{1}}{\partial z}-A_{1} B_{1}\right) g_{1}\right)}{2 \overline{\partial_{z} \Phi}}\right. & \left.+\left(2 \frac{\partial}{\partial z}+B_{1}\right)\left(2 \frac{\partial}{\partial \bar{z}}+A_{1}\right) \frac{e_{2}\left(g_{1}+\widehat{g}_{1} / \tau\right)}{2 \overline{\partial_{z} \Phi}}\right)-M_{6}(\bar{z}) e^{\mathcal{B}_{1}}
\end{aligned}
$$

Here $M_{5}(z), M_{6}(\bar{z})$ are polynomials chosen such that

$$
\left.g_{5}\right|_{\mathcal{H}}=\left.g_{6}\right|_{\mathcal{H}}=\left.\nabla g_{5}\right|_{\mathcal{H}}=\left.\nabla g_{6}\right|_{\mathcal{H}}=0 \text {. }
$$

Using Proposition 2.3 we introduce functions $a_{2,0}, d_{2,0} \in W_{4}^{1}(\Omega)$ (holomorphic and antiholomorphic respectively) such that

$$
a_{2,0} e^{\mathcal{A}_{1}}+d_{2,0} e^{\mathcal{B}_{1}}=\frac{g_{5}}{2 \partial_{z} \Phi}+\frac{g_{6}}{2 \overline{\partial_{z} \Phi}} \quad \text { on } \Gamma_{0} .
$$

Next we claim that

$$
\left\|\mathcal{R}_{-\tau, A_{1}}\left\{e_{1} g_{6}\right\}\right\|_{H^{1}\left(\Gamma_{0}\right)}+\left\|\widetilde{\mathcal{R}}_{\tau, B_{1}}\left\{e_{1} g_{5}\right\}\right\|_{H^{1}\left(\Gamma_{0}\right)}=o(1 / \tau) \quad \text { as }|\tau| \rightarrow+\infty .
$$

To see this, let us introduce the function $\mathcal{F}$ with domain $\Gamma_{0}$ :

$$
\begin{aligned}
\mathcal{F} & =2 e^{-\mathcal{A}_{1}} e^{\tau(\Phi-\bar{\Phi})} \mathcal{R}_{-\tau, A_{1}}\left\{e_{1} g_{6}\right\} \\
& =\partial_{\bar{z}}^{-1}\left(e_{1} e^{-\mathcal{A}_{1}+\tau(\Phi-\bar{\Phi})} \frac{T_{B_{1}}\left(\left(q_{1}-2 \frac{\partial B_{1}}{\partial \bar{z}}-A_{1} B_{1}\right) g_{2}\right)-M_{6} e^{\mathcal{B}_{1}}}{2 \overline{\partial_{z} \Phi}}\right) .
\end{aligned}
$$

Denoting

we have

$$
r(x)=e^{\mathcal{A}_{1}} \frac{T_{B_{1}}\left(\left(q_{1}-2 \frac{\partial B_{1}}{\partial \bar{z}}-A_{1} B_{1}\right) g_{2}\right)-M_{6}(\bar{z}) e^{\mathcal{B}_{1}}}{2 \overline{\partial_{z} \Phi}}
$$

$$
\begin{aligned}
\mathcal{F}(x) & =-\frac{1}{\pi} \int_{\Omega} \frac{e_{1}(x) r(x) e^{2 i \tau \psi}}{\bar{\zeta}-\bar{z}} d \xi_{1} d \xi_{2} \\
& =\frac{1}{2 i \pi \tau} \int_{\Omega} \sum_{k=1}^{2} \frac{\partial}{\partial x_{k}}\left(\frac{\partial \psi}{\partial x_{k}} \frac{e_{1}(x)}{|\nabla \psi|^{2}} \frac{r(x)}{\bar{\zeta}-\bar{z}}\right) e^{2 i \tau \psi} d \xi_{1} d \xi_{2} .
\end{aligned}
$$

Since $\sum_{k=1}^{2} \frac{\partial}{\partial x_{k}}\left(\frac{\frac{\partial \psi}{\partial x_{k}}}{|\nabla \psi|^{2}} \frac{e_{1}(x) r(x)}{\bar{\zeta}-\bar{z}}\right) \in L^{1}(\Omega)$, we have $\mathcal{F}=o(1 / \tau)$. This proves $(4.22)$. 
Now we finish the construction of $a_{2, \tau}(z)$ and $d_{2, \tau}(\bar{z})$ by setting

$$
\begin{aligned}
& d_{2, \tau}(\bar{z})=d_{2,0}(\bar{z})+\frac{1}{2}\left(d_{2,+}(\bar{z}) \Lambda_{+}(\tau, \widetilde{x})+d_{2,-}(\bar{z}) \Lambda_{-}(\tau, \widetilde{x})\right), \\
& a_{2, \tau}(z)=a_{2,0}(z)+\frac{1}{2}\left(a_{2,+}(z) \Lambda_{+}(\tau, \widetilde{x})+a_{2,-}(z) \Lambda_{-}(\tau, \widetilde{x})\right),
\end{aligned}
$$

where $a_{2, \pm}, d_{2, \pm}$ satisfy (4.15). In order to complete the construction of a solution to (4.1), we define $u_{0}$ as the solution to the inhomogeneous problem

$$
\begin{aligned}
L_{1}(x, D)\left(u_{0} e^{\tau \varphi}\right) & =h_{1} e^{\tau \varphi} & & \text { in } \Omega, \\
u_{0} e^{\tau \varphi} & =e^{\tau \varphi} \mathbf{m}_{1} & & \text { on } \Gamma_{0},
\end{aligned}
$$

where

$$
\begin{aligned}
h_{1}(\tau) & =-e^{-\tau \varphi} L_{1}(x, D)\left(a_{\tau} e^{\mathcal{A}_{1}+\tau \Phi}+d_{\tau} e^{\mathcal{B}_{1}+\tau \bar{\Phi}}+u_{11} e^{\tau \varphi}+u_{-1} e^{\tau \varphi}\right), \\
\mathbf{m}_{1} & =-\left.e^{-\tau \varphi}\left(a_{\tau} e^{\mathcal{A}_{1}+\tau \Phi}+d_{\tau} e^{\mathcal{B}_{1}+\tau \bar{\Phi}}+u_{11} e^{\tau \varphi}+u_{-1} e^{\tau \varphi}\right)\right|_{\Gamma_{0}} .
\end{aligned}
$$

Observe that by (4.17)-(4.19), we can represent $h_{1}(\tau)$ in the form $h_{1}(\tau)=$ $h_{11}+h_{12}$, where

$$
\left\|h_{11}\right\|_{L^{4}(\Omega)}=O\left(1 / \tau^{2}\right), \quad\left\|h_{12}\right\|_{L^{4}(\Omega)}=o(1 / \tau) \quad \text { as }|\tau| \rightarrow+\infty,
$$

and for some positive $\epsilon$,

and

$$
\operatorname{supp} h_{11} \subset \mathcal{O}_{\epsilon}, \quad \operatorname{dist}\left(\operatorname{supp} h_{12}, \partial \Omega\right)>0
$$

$$
\left\|u_{0}\right\|_{H^{1 / 2}\left(\Gamma_{0}\right)}=o\left(1 / \tau^{2}\right) \quad \text { as }|\tau| \rightarrow+\infty
$$

by (4.8), (4.14), (4.15) and (4.21).

By Propositions 2.6 and 3.8, there exists a solution to $(4.23),(4.25)$ such that

$$
\frac{1}{\sqrt{|\tau|}}\left\|u_{0}\right\|_{H^{1}(\Omega)}+\sqrt{|\tau|}\left\|u_{0}\right\|_{L^{2}(\Omega)}+\left\|u_{0}\right\|_{H^{1, \tau}\left(\mathcal{O}_{\epsilon}\right)}=o(1 / \tau) \quad \text { as }|\tau| \rightarrow+\infty .
$$

\section{§4.1. Complex geometrical optics solutions for the adjoint operator}

We now construct complex geometrical optics solutions for the adjoint operator. This parallels the previous construction since the adjoint has a similar form.

Consider the operator $L_{2}(x, D)=4 \frac{\partial}{\partial z} \frac{\partial}{\partial \bar{z}}+2 A_{2} \frac{\partial}{\partial z}+2 B_{2} \frac{\partial}{\partial \bar{z}}+q_{2}$. Its adjoint has the form

$$
\begin{aligned}
L_{2}(x, D)^{*} & =4 \frac{\partial}{\partial z} \frac{\partial}{\partial \bar{z}}-2 \overline{A_{2}} \frac{\partial}{\partial \bar{z}}-2 \overline{B_{2}} \frac{\partial}{\partial z}+\overline{q_{2}}-2 \frac{\partial \overline{A_{2}}}{\partial \bar{z}}-\frac{\partial \overline{B_{2}}}{\partial z} \\
& =\left(2 \frac{\partial}{\partial z}-\overline{A_{2}}\right)\left(2 \frac{\partial}{\partial \bar{z}}-\overline{B_{2}}\right)+\overline{q_{2}}-2 \frac{\partial \overline{A_{2}}}{\partial \bar{z}}-\overline{A_{2} B_{2}} \\
& =\left(2 \frac{\partial}{\partial \bar{z}}-\overline{B_{2}}\right)\left(2 \frac{\partial}{\partial z}-\overline{A_{2}}\right)+\overline{q_{2}}-2 \frac{\partial \overline{B_{2}}}{\partial z}-\overline{A_{2} B_{2}}
\end{aligned}
$$


Next we construct solutions to the boundary value problem

$$
L_{2}(x, D)^{*} v=0 \quad \text { in } \Omega,\left.\quad v\right|_{\Gamma_{0}}=0,
$$

of the form

$$
v(x)=b_{\tau}(z) e^{\mathcal{B}_{2}-\tau \Phi}+c_{\tau}(\bar{z}) e^{\mathcal{A}_{2}-\tau \bar{\Phi}}+v_{11} e^{-\tau \varphi}+v_{12} e^{-\tau \varphi},\left.\quad v\right|_{\Gamma_{0}}=0 .
$$

Here $\mathcal{A}_{2}, \mathcal{B}_{2} \in C^{6+\alpha}(\bar{\Omega})$ satisfy

$$
2 \frac{\partial \mathcal{A}_{2}}{\partial z}=\overline{A_{2}} \quad \text { in } \Omega,\left.\quad \operatorname{Im} \mathcal{A}_{2}\right|_{\Gamma_{0}}=0, \quad 2 \frac{\partial \mathcal{B}_{2}}{\partial \bar{z}}=\overline{B_{2}} \quad \text { in } \Omega,\left.\quad \operatorname{Im} \mathcal{B}_{2}\right|_{\Gamma_{0}}=0,
$$

and $b_{\tau}(z)=b(z)+b_{1}(z) / \tau+b_{2, \tau}(z) / \tau^{2}, c_{\tau}(\bar{z})=c(\bar{z})+c_{1}(\bar{z}) / \tau+c_{2, \tau}(\bar{z}) / \tau^{2}$,

$$
\begin{aligned}
& b, c \in C^{5+\alpha}(\bar{\Omega}), \quad \frac{\partial b}{\partial \bar{z}}=0 \quad \text { in } \Omega, \quad \frac{\partial c}{\partial z}=0 \quad \text { in } \Omega, \\
& \left.\left(b e^{\mathcal{B}_{2}}+c e^{\mathcal{A}_{2}}\right)\right|_{\Gamma_{0}}=0, \\
& \left.\frac{\partial^{k} b}{\partial z^{k}}\right|_{\mathcal{H} \backslash\{\widetilde{x}\}}=\left.\frac{\partial^{k} c}{\partial \bar{z}^{k}}\right|_{\mathcal{H} \backslash\{\widetilde{x}\}}=0 \quad \forall k \in\{0, \ldots, 5\}, \quad b(\widetilde{x}) \neq 0, c(\widetilde{x}) \neq 0 .
\end{aligned}
$$

The existence of $b$ and $c$ is given by Proposition 7.2. Denote

$$
\begin{aligned}
& g_{3}=P_{-\overline{B_{2}}}\left(\left(\bar{q}_{2}-2 \frac{\partial \overline{A_{2}}}{\partial \bar{z}}-\overline{A_{2} B_{2}}\right) b e^{\mathcal{B}_{2}}\right)-M_{3}(z) e^{\mathcal{B}_{2}} \\
& g_{4}=T_{-\overline{A_{2}}}\left(\left(\bar{q}_{2}-2 \frac{\partial \overline{B_{2}}}{\partial z}-\overline{A_{2} B_{2}}\right) c e^{\mathcal{A}_{2}}\right)-M_{4}(\bar{z}) e^{\mathcal{A}_{2}},
\end{aligned}
$$

where the polynomials $M_{3}(z), M_{4}(\bar{z})$ are chosen such that

$$
\left.\frac{\partial^{k} g_{3}}{\partial z^{k}}\right|_{\mathcal{H}}=\left.\frac{\partial^{k} g_{4}}{\partial \bar{z}^{k}}\right|_{\mathcal{H}}=0 \quad \forall k \in\{0, \ldots, 6\} .
$$

By (4.34) and (4.33), we have

$$
\left.\frac{\partial^{k+j} g_{3}}{\partial z^{k} \partial \bar{z}^{j}}\right|_{\mathcal{H} \backslash\{\widetilde{x}\}}=\left.\frac{\partial^{k+j} g_{4}}{\partial z^{k} \partial \bar{z}^{j}}\right|_{\mathcal{H} \backslash\{\widetilde{x}\}}=0 \quad \forall k+j \leq 6 .
$$

Observe that by (4.35), $g_{3} / \partial_{z} \Phi, g_{4} / \overline{\partial_{z} \Phi} \in C^{4+\alpha}(\bar{\Omega})$. Using Proposition 2.3, we introduce a holomorphic function $b_{1}(z) \in C^{2}(\bar{\Omega})$ and an antiholomorphic function $c_{1}(\bar{z}) \in C^{2}(\bar{\Omega})$ such that

$$
b_{1} e^{\mathcal{B}_{2}}+c_{1} e^{\mathcal{A}_{2}}=\frac{e_{2} g_{3}}{2 \partial_{z} \Phi}+\frac{e_{2} g_{4}}{2 \overline{\partial_{z} \Phi}} \quad \text { on } \Gamma_{0} .
$$

Let

$$
\widehat{g}_{3}=P_{-\overline{B_{2}}}\left(\left(\bar{q}_{2}-2 \frac{\partial \overline{A_{2}}}{\partial \bar{z}}-\overline{A_{2} B_{2}}\right) b_{1} e^{\mathcal{B}_{2}}\right)-\widehat{M}_{3}(z) e^{\mathcal{B}_{2}}
$$


1010

$$
\widehat{g}_{4}=T_{-} \overline{A_{2}}\left(\left(\bar{q}_{2}-2 \frac{\partial \overline{B_{2}}}{\partial z}-\overline{A_{2} B_{2}}\right) c_{1} e^{\mathcal{A}_{2}}\right)-\widehat{M}_{4}(\bar{z}) e^{\mathcal{A}_{2}},
$$

where the polynomials $\widehat{M}_{3}(z), \widehat{M}_{4}(\bar{z})$ are chosen such that

$$
\left.\frac{\partial^{k} \widehat{g}_{3}}{\partial z^{k}}\right|_{\mathcal{H}}=\left.\frac{\partial^{k} \widehat{g}_{4}}{\partial \bar{z}^{k}}\right|_{\mathcal{H}}=0 \quad \forall k \in\{0, \ldots, 3\} .
$$

The function $v_{11}$ is defined by

$$
\begin{aligned}
v_{11}= & -e^{-i \tau \psi} \widetilde{\mathcal{R}}_{-\tau,-\overline{A_{2}}}\left\{e_{1}\left(g_{3}+\widehat{g}_{3} / \tau\right)\right\}+\frac{e^{-i \tau \psi} e_{2}\left(g_{3}+\widehat{g}_{3} / \tau\right)}{2 \tau \partial_{z} \Phi} \\
& -e^{i \tau \psi} \mathcal{R}_{\tau,-\overline{B_{2}}}\left\{e_{1}\left(g_{4}+\widehat{g}_{4} / \tau\right)\right\}+\frac{e^{i \tau \psi} e_{2}\left(g_{4}+\widehat{g}_{4} / \tau\right)}{2 \tau \overline{\partial_{z} \Phi}} .
\end{aligned}
$$

Here we set

$$
\begin{aligned}
\mathcal{R}_{\tau,-\overline{B_{2}}}\{g\} & =\frac{1}{2} e^{\mathcal{B}_{2}} e^{\tau(\bar{\Phi}-\Phi)} \partial_{\bar{z}}^{-1}\left(g e^{-\mathcal{B}_{2}} e^{\tau(\Phi-\bar{\Phi})}\right), \\
\widetilde{\mathcal{R}}_{-\tau,-\overline{A_{2}}}\{g\} & =\frac{1}{2} e^{\mathcal{A}_{2}} e^{\tau(\Phi-\bar{\Phi})} \partial_{z}^{-1}\left(g e^{-\mathcal{A}_{2}} e^{\tau(\bar{\Phi}-\Phi)}\right),
\end{aligned}
$$

provided that $A_{2}, B_{2}, \mathcal{A}_{2}, \mathcal{B}_{2}$ satisfy (4.30). By Proposition 8.1 the following asymptotic formulae hold:

$$
\begin{aligned}
& \left.(4.39) \widetilde{\mathcal{R}}_{-\tau,-\overline{A_{2}}}\left\{e_{1} g_{3}\right\}\right|_{\partial \Omega}=\frac{e^{\mathcal{A}_{2}+2 \tau i \psi}}{2 \tau^{2}}\left(\frac{\Lambda_{-}(\tau, \widetilde{x}) r_{1}(\widetilde{x})}{(\bar{z}-\overline{\widetilde{z}})^{2}}+\frac{\Lambda_{-}(\tau, \widetilde{x}) t_{1}(\widetilde{x})}{\overline{\widetilde{z}}-\bar{z}}\right)+\widetilde{\mathcal{W}}_{2, \tau}, \\
& \left.(4.40) \quad \mathcal{R}_{\tau,-\overline{B_{2}}}\left\{e_{1} g_{4}\right\}\right|_{\partial \Omega}=\frac{e^{\mathcal{B}_{2}-2 \tau i \psi}}{2 \tau^{2}}\left(\frac{\Lambda_{+}(\tau, \widetilde{x}) \widetilde{r}_{1}(\widetilde{x})}{(z-\widetilde{z})^{2}}+\frac{\Lambda_{+}(\tau, \widetilde{x}) \widetilde{t}_{1}(\widetilde{x})}{\widetilde{z}-z}\right)+\widetilde{\mathcal{W}}_{1, \tau},
\end{aligned}
$$

where

(4.41) $\quad r_{1}(\widetilde{x})=\frac{\partial_{\bar{z}} \widetilde{g}_{3}(\widetilde{x})}{\overline{\partial_{z}^{2} \Phi(\widetilde{x})}}, \quad t_{1}(\widetilde{x})=\frac{1}{2}\left(\frac{\partial_{z} \widetilde{g}_{3}(\widetilde{x})}{\overline{\partial_{z}^{2} \Phi(\widetilde{x})}} \frac{\partial_{\bar{z}}^{3} \bar{\Phi}(\widetilde{x})}{\partial_{\bar{z}}^{2} \bar{\Phi}(\widetilde{x})}+\frac{\partial_{z}^{2} \widetilde{g}_{3}(\widetilde{x})}{\partial_{z}^{2} \Phi(\widetilde{x})}-\frac{\partial_{\bar{z}}^{2} \widetilde{g}_{3}(\widetilde{x})}{\partial_{z}^{2} \Phi(\widetilde{x})}\right)$,

(4.42) $\quad \widetilde{r}_{1}(\widetilde{x})=\frac{\partial_{z} \widetilde{g}_{4}(\widetilde{x})}{\partial_{z}^{2} \Phi(\widetilde{x})}, \quad \widetilde{t}_{1}(\widetilde{x})=\frac{1}{2}\left(\frac{\partial_{z} \widetilde{g}_{4}(\widetilde{x})}{\partial_{z}^{2} \Phi(\widetilde{x})} \frac{\partial_{z}^{3} \Phi(\widetilde{x})}{\partial_{z}^{2} \Phi(\widetilde{x})}-\frac{\partial_{z}^{2} \widetilde{g}_{4}(\widetilde{x})}{\partial_{z}^{2} \Phi(\widetilde{x})}+\frac{\partial_{z}^{2} \widetilde{g}_{4}(\widetilde{x})}{\partial_{z}^{2} \Phi(\widetilde{x})}\right)$,

where we set $\widetilde{g}_{3}=e^{-\mathcal{A}_{2}} g_{3}$ and $\widetilde{g}_{4}=e^{-\mathcal{B}_{2}} g_{4}$. Here the functions $\widetilde{\mathcal{W}}_{\tau, 1}, \widetilde{\mathcal{W}}_{\tau, 2} \in$ $H^{1 / 2}\left(\Gamma_{0}\right)$ satisfy

$$
\left\|\widetilde{\mathcal{W}}_{\tau, 1}\right\|_{H^{1 / 2}\left(\Gamma_{0}\right)}+\left\|\widetilde{\mathcal{W}}_{\tau, 2}\right\|_{H^{1 / 2}\left(\Gamma_{0}\right)}=o\left(1 / \tau^{2}\right) \quad \text { as }|\tau| \rightarrow+\infty .
$$

Using Proposition 2.3 we define holomorphic functions $b_{2, \pm}(z) \in C^{2}(\bar{\Omega})$ and antiholomorphic $c_{2, \pm}(\bar{z}) \in C^{2}(\bar{\Omega})$ such that

$$
b_{2, \pm} e^{\mathcal{B}_{2}}+c_{2, \pm} e^{\mathcal{A}_{2}}=\widetilde{p}_{ \pm} \quad \text { on } \Gamma_{0},
$$


where $\widetilde{p}_{ \pm}$are defined by

$$
\widetilde{p}_{+}(x)=e^{\mathcal{B}_{2}}\left(\frac{\widetilde{r}_{1}(\widetilde{x})}{(z-\widetilde{z})^{2}}+\frac{\widetilde{t}_{1}(\widetilde{x})}{\widetilde{z}-z}\right), \quad \widetilde{p}_{-}(x)=e^{\mathcal{A}_{2}}\left(\frac{r_{1}(\widetilde{x})}{(\bar{z}-\overline{\widetilde{z}})^{2}}+\frac{t_{1}(\widetilde{x})}{\overline{\widetilde{z}}-\bar{z}}\right) .
$$

Similarly to (4.17), there exist positive numbers $\epsilon$ and $\epsilon^{\prime}$ such that

$$
\begin{aligned}
L_{2}(x, D)^{*} & \left(\left(b+b_{1} / \tau\right) e^{\mathcal{B}_{2}-\tau \Phi}+\left(\bar{b}+c_{1} / \tau\right) e^{\mathcal{A}_{2}-\tau \bar{\Phi}}+v_{11} e^{-\tau \varphi}\right) \\
= & \frac{g_{3} e^{-\tau \Phi}}{2 \tau \partial_{z} \Phi}\left(\overline{q_{2}}-2 \frac{\partial \overline{B_{2}}}{\partial z}-\overline{A_{2} B_{2}}\right)-\frac{g_{4} e^{-\tau \bar{\Phi}}}{2 \tau \overline{\partial_{z} \Phi}}\left(\overline{q_{2}}-2 \frac{\partial \overline{A_{2}}}{\partial \bar{z}}-\overline{A_{2} B_{2}}\right) \\
& +\left(2 \frac{\partial}{\partial \bar{z}}-\overline{B_{2}}\right)\left(2 \frac{\partial}{\partial z}-\overline{A_{2}}\right) \frac{g_{3} e^{-\tau \Phi}}{2 \tau \partial_{z} \Phi} \\
& +\left(2 \frac{\partial}{\partial z}-\overline{A_{2}}\right)\left(2 \frac{\partial}{\partial \bar{z}}-\overline{B_{2}}\right) \frac{g_{4} e^{-\tau \bar{\Phi}}}{2 \tau \overline{\partial_{z} \Phi}} \\
& +e^{-\tau \varphi} \chi_{\Omega \backslash \mathcal{O}_{\epsilon}^{\prime} O_{L^{4}}(\Omega)}(1 / \tau)+e^{-\tau \varphi} \chi_{\mathcal{O}_{\epsilon}} O_{L^{4}(\Omega)}\left(1 / \tau^{2}\right) .
\end{aligned}
$$

We are looking for $v_{12}$ in the form $v_{12}=v_{0}+v_{-1}$. The function $v_{-1}$ is given by

$$
\begin{aligned}
v_{-1}= & -\frac{e^{\tau i \psi}}{\tau} \mathcal{R}_{\tau,-\overline{B_{2}}}\left\{e_{1} g_{8}\right\}-\frac{e^{-\tau i \psi}}{\tau} \widetilde{\mathcal{R}}_{-\tau,-\overline{A_{2}}}\left\{e_{1} g_{7}\right\}+\frac{e_{2} g_{7} e^{-i \tau \psi}}{2 \tau^{2} \partial_{z} \Phi} \\
& +\frac{e_{2} g_{8} e^{i \tau \psi}}{2 \tau^{2} \overline{\partial_{z} \Phi}},
\end{aligned}
$$

where

$$
\begin{aligned}
g_{7}= & P_{-\overline{B_{2}}}\left(\frac{\left(\overline{q_{2}}-2 \frac{\partial \overline{B_{2}}}{\partial z}-\overline{A_{2} B_{2}}\right) g_{3}}{2 \partial_{z} \Phi}+\left(2 \frac{\partial}{\partial \bar{z}}-\overline{B_{2}}\right)\left(2 \frac{\partial}{\partial z}-\overline{A_{2}}\right) \frac{g_{3}}{2 \partial_{z} \Phi}\right) \\
& -M_{7}(z) e^{\mathcal{B}_{2}}, \\
g_{8}= & T_{-\overline{A_{2}}}\left(\frac{\left(\overline{q_{2}}-2 \frac{\partial \overline{A_{2}}}{\partial \bar{z}}-\overline{A_{2} B_{2}}\right) g_{4}}{2 \overline{\partial_{z} \Phi}}+\left(2 \frac{\partial}{\partial z}-\overline{A_{2}}\right)\left(2 \frac{\partial}{\partial \bar{z}}-\overline{B_{2}}\right) \frac{g_{4}}{2 \overline{\partial_{z} \Phi}}\right) \\
& -M_{8}(\bar{z}) e^{\mathcal{A}_{2}}
\end{aligned}
$$

and $M_{7}(z), M_{8}(\bar{z})$ are polynomials chosen such that

$$
\left.g_{7}\right|_{\mathcal{H}}=\left.g_{8}\right|_{\mathcal{H}}=\left.\nabla g_{7}\right|_{\mathcal{H}}=\left.\nabla g_{8}\right|_{\mathcal{H}}=0 .
$$

Using Proposition 2.3, we introduce $b_{2,0}, c_{2,0} \in C^{2}(\bar{\Omega})$ such that

$$
b_{2,0} e^{\mathcal{B}_{2}}+c_{2,0} e^{\mathcal{A}_{2}}=\frac{g_{7}}{2 \overline{\partial_{z} \Phi}}+\frac{g_{8}}{2 \partial_{z} \Phi} \quad \text { on } \Gamma_{0}
$$

Similarly to (4.22), we have

$$
\frac{1}{\tau}\left\|\mathcal{R}_{\tau,-\overline{B_{2}}}\left\{e_{1} g_{8}\right\}\right\|_{H^{1}\left(\Gamma_{0}\right)}+\frac{1}{\tau}\left\|\widetilde{\mathcal{R}}_{-\tau,-\overline{A_{2}}}\left\{e_{1} g_{7}\right\}\right\|_{H^{1}\left(\Gamma_{0}\right)}=o\left(1 / \tau^{2}\right) \quad \text { as }|\tau| \rightarrow+\infty .
$$


Now we finish the construction of $b_{2, \tau}(z)$ and $c_{2, \tau}(\bar{z})$ by setting

$$
\begin{aligned}
& b_{2, \tau}(z)=b_{2,0}(z)+\frac{1}{2}\left(b_{2,+}(z) \Lambda_{+}(\tau, \widetilde{x})+b_{2,-}(z) \Lambda_{-}(\tau, \widetilde{x})\right), \\
& c_{2, \tau}(\bar{z})=c_{2,0}(\bar{z})+\frac{1}{2}\left(c_{2,+}(\bar{z}) \Lambda_{+}(\tau, \widetilde{x})+c_{2,-}(\bar{z}) \Lambda_{-}(\tau, \widetilde{x})\right),
\end{aligned}
$$

where $b_{2,+}, c_{2,-}$ are defined in (4.44).

Consider the boundary value problem

$$
\begin{aligned}
L_{2}(x, D)^{*}\left(e^{-\tau \varphi} v_{0}\right) & =h_{2} e^{-\tau \varphi} \quad \text { in } \Omega, \\
\left.e^{-\tau \varphi} v_{0}\right|_{\Gamma_{0}} & =\mathbf{m}_{2} e^{-\tau \varphi},
\end{aligned}
$$

where

$$
\begin{aligned}
h_{2} & =-e^{\tau \varphi} L_{2}(x, D)^{*}\left(b_{\tau} e^{\mathcal{B}_{2}-\tau \Phi}+c_{\tau} e^{\mathcal{A}_{2}-\tau \bar{\Phi}}+v_{11} e^{-\tau \varphi}+v_{-1} e^{-\tau \varphi}\right), \\
\mathbf{m}_{2} & =-e^{\tau \varphi}\left(b_{\tau} e^{\mathcal{A}_{2}-\tau \Phi}+c_{\tau} e^{\mathcal{B}_{2}-\tau \bar{\Phi}}+v_{11} e^{-\tau \varphi}+v_{-1} e^{-\tau \varphi}\right) .
\end{aligned}
$$

By (4.45)-(4.47) we can represent $h_{2}$ in the form $h_{2}=h_{21}+h_{22}$ where for some positive $\epsilon$,

$$
\operatorname{supp} h_{21} \subset \mathcal{O}_{\epsilon}, \quad \operatorname{dist}\left(\operatorname{supp} h_{22}, \partial \Omega\right)>0 .
$$

The norms of the functions $h_{2 j}$ are estimated as

$$
\left\|h_{21}\right\|_{L^{4}(\Omega)}=O\left(1 / \tau^{2}\right), \quad\left\|h_{22}\right\|_{L^{4}(\Omega)}=o(1 / \tau) \quad \text { as }|\tau| \rightarrow+\infty .
$$

By (4.51), (4.52), (4.44), (4.43) and (4.31), we have

$$
\left\|v_{0}\right\|_{H^{1 / 2}\left(\Gamma_{0}\right)}=o\left(1 / \tau^{2}\right) \quad \text { as }|\tau| \rightarrow+\infty .
$$

Thanks to (4.55) and (4.56), by Propositions 2.6 and 3.8, for sufficiently small positive $\epsilon$ there exists a solution to problem (4.53), (4.54) such that

$$
\frac{1}{\sqrt{|\tau|}}\left\|v_{0}\right\|_{H^{1}(\Omega)}+\sqrt{|\tau|}\left\|v_{0}\right\|_{L^{2}(\Omega)}+\left\|v_{0}\right\|_{H^{1, \tau}\left(\mathcal{O}_{\epsilon}\right)}=o(1 / \tau) \quad \text { as }|\tau| \rightarrow+\infty .
$$

\section{$\S 5$. Proof of Theorem 1.3}

Let $u_{1}$ be a complex geometrical optics solution as in (4.2). Let $u_{2}$ be a solution to the boundary value problem

$$
L_{2}(x, D) u_{2}=0 \quad \text { in } \Omega,\left.\quad u_{2}\right|_{\partial \Omega}=\left.u_{1}\right|_{\partial \Omega},\left.\quad \frac{\partial u_{2}}{\partial \nu}\right|_{\widetilde{\Gamma}}=\left.\frac{\partial u_{1}}{\partial \nu}\right|_{\widetilde{\Gamma}} .
$$


Setting $u=u_{1}-u_{2}$ and $q=q_{1}-q_{2}$, we have

$$
\begin{aligned}
& L_{2}(x, D) u+2\left(A_{1}-A_{2}\right) \frac{\partial u_{1}}{\partial z}+2\left(B_{1}-B_{2}\right) \frac{\partial u_{1}}{\partial \bar{z}}+q u_{1}=0 \quad \text { in } \Omega, \\
& \left.u\right|_{\partial \Omega}=0,\left.\quad \frac{\partial u}{\partial \nu}\right|_{\widetilde{\Gamma}}=0 .
\end{aligned}
$$

Let $v$ be a solution to (4.28) in the form (4.29). Taking the scalar product of (5.2) with $v$ in $L^{2}(\Omega)$ we obtain

$$
0=\int_{\Omega}\left(2\left(A_{1}-A_{2}\right) \frac{\partial u_{1}}{\partial z}+2\left(B_{1}-B_{2}\right) \frac{\partial u_{1}}{\partial \bar{z}}+q u_{1}\right) \bar{v} d x
$$

Our goal is to obtain the asymptotic formula for the right-hand side of (5.4). We have

Proposition 5.1. The following asymptotic formula is valid as $|\tau| \rightarrow+\infty$ :

$$
\begin{aligned}
I_{0}:=\left(q u_{1}, v\right)_{L^{2}(\Omega)}=\int_{\Omega}\left(q a \bar{c} e^{\left(\mathcal{A}_{1}+\overline{\mathcal{A}_{2}}\right)}+q d \bar{b} e^{\left(\mathcal{B}_{1}+\overline{\mathcal{B}_{2}}\right)}\right) d x \\
+\int_{\Omega}\left(\frac{q}{\tau}\left(a_{1} b+a \overline{c_{1}}\right) e^{\left(\mathcal{A}_{1}-\overline{\mathcal{B}_{2}}\right)}+\frac{q}{\tau}\left(\bar{a} \overline{b_{1}}+\bar{b} d_{1}\right) e^{\left(\mathcal{A}_{2}-\overline{\mathcal{B}_{1}}\right)}\right) d x \\
+\frac{1}{\tau} \int_{\Omega} q\left(\frac{a \overline{g_{4}} e^{\mathcal{A}_{1}}}{2 \partial_{z} \Phi}-\frac{\bar{c} g_{2} e^{\overline{\mathcal{A}_{2}}}}{2 \partial_{z} \Phi}-\frac{\bar{b} g_{1} e^{\overline{\mathcal{B}_{2}}}}{2 \overline{\partial_{z} \Phi}}+\frac{d \overline{g_{3}} e^{\mathcal{B}_{1}}}{2 \overline{\partial_{z} \Phi}}\right) d x \\
+2 \pi \frac{(q a \bar{b})(\widetilde{x}) \Lambda_{+}(\tau, \widetilde{x}) e^{\left(\mathcal{A}_{1}+\overline{\mathcal{B}_{2}}\right)(\widetilde{x})}+(q d \bar{c})(\widetilde{x}) \Lambda_{-}(\tau, \widetilde{x}) e^{\left(\mathcal{B}_{1}+\overline{\mathcal{A}_{2}}\right)(\widetilde{x})}}{\tau} \\
+\frac{1}{2 \tau i} \int_{\partial \Omega} q a \bar{b} e^{\mathcal{A}_{1}+\overline{\mathcal{B}_{2}}+2 \tau i \psi} \frac{(\nu, \nabla \psi)}{|\nabla \psi|^{2}} d \sigma \\
-\frac{1}{2 \tau i} \int_{\partial \Omega} q d \bar{c} e^{\mathcal{B}_{1}+\overline{\mathcal{A}_{2}}-2 \tau i \psi} \frac{(\nu, \nabla \psi)}{|\nabla \psi|^{2}} d \sigma+o(1 / \tau) .
\end{aligned}
$$

Proof. By (4.2), (4.9), (4.27) and Proposition 3.4, we have

$$
\begin{aligned}
u_{1}(x)= & \left(a(z)+a_{1}(z) / \tau\right) e^{\mathcal{A}_{1}+\tau \Phi}+\left(d(\bar{z})+d_{1}(\bar{z}) / \tau\right) e^{\mathcal{B}_{1}+\tau \bar{\Phi}} \\
& -\frac{g_{1} e^{\tau \bar{\Phi}}}{2 \tau \overline{\partial_{z} \Phi}}-\frac{g_{2} e^{\tau \Phi}}{2 \tau \partial_{z} \Phi}+e^{\tau \varphi} O_{L^{2}(\Omega)}(1 / \tau) .
\end{aligned}
$$

Using (4.29), (4.38), (4.57) and Proposition 3.4, we obtain

$$
\begin{aligned}
v(x)= & \left(b(z)+b_{1}(z) / \tau\right) e^{\mathcal{B}_{2}-\tau \Phi}+\left(c(\bar{z})+c_{1}(\bar{z}) / \tau\right) e^{\mathcal{A}_{2}-\tau \bar{\Phi}} \\
& +\frac{g_{4} e^{-\tau \bar{\Phi}}}{2 \tau \overline{\partial_{z} \Phi}}+\frac{g_{3} e^{-\tau \Phi}}{2 \tau \partial_{z} \Phi}+e^{-\tau \varphi} O_{L^{2}(\Omega)}(1 / \tau)
\end{aligned}
$$


By (5.6), (5.7) and Proposition 3.3, we obtain

$$
\begin{aligned}
& \left(q u_{1}, \bar{v}\right)_{L^{2}(\Omega)}= \\
& \left(q\left(\left(a+a_{1} / \tau\right) e^{\mathcal{A}_{1}+\tau \Phi}+\left(d+d_{1} / \tau\right) e^{\mathcal{B}_{1}+\tau \bar{\Phi}}-\frac{g_{1} e^{\tau \bar{\Phi}}}{2 \tau \overline{\partial_{z} \Phi}}-\frac{g_{2} e^{\tau \Phi}}{2 \tau \partial_{z} \Phi}+e^{\tau \varphi} o_{L^{2}(\Omega)}(1 / \tau)\right)\right. \\
& \left.\left(b+b_{1} / \tau\right) e^{\mathcal{B}_{2}-\tau \Phi}+\left(c+c_{1} / \tau\right) e^{\mathcal{A}_{2}-\tau \bar{\Phi}}+\frac{g_{4} e^{-\tau \bar{\Phi}}}{2 \tau \overline{\partial_{z} \Phi}}+\frac{g_{3} e^{-\tau \Phi}}{2 \tau \partial_{z} \Phi}+e^{-\tau \varphi} O_{L^{2}(\Omega)}(1 / \tau)\right) \\
& =\int_{\Omega}\left(q\left(d \bar{b}+\frac{1}{\tau}\left(d_{1} \bar{b}+d \bar{b}_{1}\right)\right) e^{\mathcal{B}_{1}+\overline{\mathcal{B}_{2}}}+q\left(a \bar{c}+\frac{1}{\tau}\left(a \overline{c_{1}}+a_{1} \bar{c}\right)\right) e^{\mathcal{A}_{1}+\overline{\mathcal{A}_{2}}}\right) d x \\
& \quad+\frac{1}{\tau} \int_{\Omega} q\left(\frac{a \overline{g_{4}} e^{\mathcal{A}_{1}}}{2 \partial_{z} \Phi}-\frac{\bar{c} g_{2} e^{\overline{\mathcal{A}_{2}}}}{2 \partial_{z} \Phi}-\frac{\bar{b} g_{1} e^{\overline{\mathcal{B}_{2}}}}{2 \overline{\partial_{z} \Phi}}+\frac{d \overline{g_{3}} e^{\mathcal{B}_{1}}}{2 \overline{\partial_{z} \Phi}}\right) d x \\
& \quad+\int_{\Omega} q\left(a \bar{b} e^{\mathcal{A}_{1}+\overline{\mathcal{B}_{2}}+\tau(\Phi-\bar{\Phi})}+d \bar{c} e^{\mathcal{B}_{1}+\overline{\mathcal{A}_{2}}+\tau(\bar{\Phi}-\Phi)}\right) d x+o(1 / \tau)
\end{aligned}
$$

Applying the stationary phase argument to the last integral, we finish the proof.

We set

$$
\begin{aligned}
& \mathcal{U}(x)=a_{\tau}(z) e^{\mathcal{A}_{1}(x)+\tau \Phi(z)}+d_{\tau}(\bar{z}) e^{\mathcal{B}_{1}(x)+\tau \overline{\Phi(z)}}, \\
& \mathcal{V}(x)=b_{\tau}(z) e^{\mathcal{B}_{2}(x)-\tau \Phi(z)}+c_{\tau}(\bar{z}) e^{\mathcal{A}_{2}(x)-\tau \overline{\Phi(z)}}
\end{aligned}
$$

Short calculations give

$$
\begin{aligned}
& I_{1}:=2\left(\left(A_{1}-A_{2}\right) \frac{\partial \mathcal{U}}{\partial z}, \mathcal{V}\right)_{L^{2}(\Omega)}\left(2\left(A_{1}-A_{2}\right)\left\{\left(\left(\frac{\partial \mathcal{A}_{1}}{\partial z}+\tau \frac{\partial \Phi}{\partial z}\right) a_{\tau}+\frac{\partial a_{\tau}}{\partial z}\right) e^{\mathcal{A}_{1}+\tau \Phi}+d_{\tau} \frac{\partial \mathcal{B}_{1}}{\partial z} e^{\mathcal{B}_{1}+\tau \Phi}\right\}\right. \\
&\left.=b_{\tau} e^{\mathcal{B}_{2}-\tau \Phi}+c_{\tau} e^{\mathcal{A}_{2}-\tau \bar{\Phi}}\right)_{L^{2}(\Omega)} \\
&=\sum_{k=1}^{3} \tau^{2-k} \kappa_{k}+\int_{\Omega}\left\{-\left(A_{1}-A_{2}\right) B_{1} d_{\tau} \overline{c_{\tau}} e^{\mathcal{B}_{1}+\overline{\mathcal{A}_{2}}-2 i \tau \psi}\right. \\
&\left.\quad-\left(A_{1}-A_{2}\right) B_{2} a_{\tau} \overline{b_{\tau}} e^{\mathcal{A}_{1}+\overline{\mathcal{B}_{2}}+2 i \tau \psi}-2 \frac{\partial}{\partial z}\left(A_{1}-A_{2}\right) a_{\tau} \overline{b_{\tau}} e^{\mathcal{A}_{1}+\overline{\mathcal{B}_{2}}+2 i \tau \psi}\right\} d x \\
&+\int_{\partial \Omega}\left(A_{1}-A_{2}\right)\left(\nu_{1}-i \nu_{2}\right) a_{\tau} \overline{b_{\tau}} e^{\mathcal{A}_{1}+\overline{\mathcal{B}_{2}}+2 i \tau \psi} d \sigma+\frac{1}{\tau} \mathcal{I}_{1}(\partial \Omega)+o(1 / \tau)
\end{aligned}
$$

and

$$
\begin{aligned}
& \text { (5.10) } \quad I_{2}:=\left(\left(B_{1}-B_{2}\right) \frac{\partial \mathcal{U}}{\partial \bar{z}}, \mathcal{V}\right)_{L^{2}(\Omega)} \\
& =\left(2\left(B_{1}-B_{2}\right)\left(a_{\tau} e^{\mathcal{A}_{1}+\tau \Phi} \frac{\partial \mathcal{A}_{1}}{\partial \bar{z}}+\frac{\partial}{\partial \bar{z}}\left(d_{\tau} e^{\mathcal{B}_{1}+\tau \bar{\Phi}}\right)\right), b_{\tau} e^{\mathcal{B}_{2}-\tau \Phi}+c_{\tau} e^{\mathcal{A}_{2}-\tau \bar{\Phi}}\right)_{L^{2}(\Omega)}
\end{aligned}
$$




$$
\begin{aligned}
= & \sum_{k=1}^{3} \tau^{2-k} \widetilde{\kappa}_{k}+\int_{\Omega} 2\left(B_{1}-B_{2}\right) \frac{\partial \mathcal{A}_{1}}{\partial \bar{z}} a_{\tau} \overline{b_{\tau}} e^{\mathcal{A}_{1}+\overline{\mathcal{B}_{2}}+2 \tau i \psi} d x \\
& -\left(2 \frac{\partial}{\partial \bar{z}}\left(B_{1}-B_{2}\right) d_{\tau} e^{\mathcal{B}_{1}+\tau \bar{\Phi}}, c_{\tau} e^{\mathcal{A}_{2}-\tau \bar{\Phi}}\right)_{L^{2}(\Omega)} \\
& -\left(2\left(B_{1}-B_{2}\right) d_{\tau} e^{\mathcal{B}_{1}+\tau \bar{\Phi}}, \frac{\partial \mathcal{A}_{2}}{\partial z} c_{\tau} e^{\mathcal{A}_{2}-\tau \bar{\Phi}}\right)_{L^{2}(\Omega)} \\
& +\int_{\partial \Omega}\left(B_{1}-B_{2}\right)\left(\nu_{1}+i \nu_{2}\right) d_{\tau} \overline{c_{\tau}} e^{\mathcal{B}_{1}+\overline{\mathcal{A}_{2}}-2 i \tau \psi} d \sigma+\frac{1}{\tau} \mathcal{I}_{2}(\partial \Omega)+o(1 / \tau) \\
= & \sum_{k=1}^{3} \tau^{2-k} \widetilde{\kappa}_{k}+\int_{\Omega}\left\{-\left(B_{1}-B_{2}\right) A_{1} a_{\tau} \overline{b_{\tau}} e^{\mathcal{A}_{1}+\overline{\mathcal{B}_{2}}+2 \tau i \psi}\right. \\
& \left.-2 \frac{\partial}{\partial \bar{z}}\left(B_{1}-B_{2}\right) d_{\tau} \overline{c_{\tau}} e^{\mathcal{B}_{1}+\overline{\mathcal{A}_{2}}-2 i \tau \psi}-\left(B_{1}-B_{2}\right) d_{\tau} \overline{c_{\tau}} A_{2} e^{\mathcal{B}_{1}+\overline{\mathcal{A}_{2}}-2 i \tau \psi}\right\} d x \\
& +\int_{\partial \Omega}\left(B_{1}-B_{2}\right)\left(\nu_{1}+i \nu_{2}\right) d_{\tau} \overline{c_{\tau}} e^{\mathcal{B}_{1}+\overline{\mathcal{A}_{2}}-2 i \tau \psi} d \sigma+\frac{1}{\tau} \mathcal{I}_{2}(\partial \Omega)+o(1 / \tau) .
\end{aligned}
$$

Here the constants $\kappa_{k}, \widetilde{\kappa}_{k}$ are independent of $\tau$ but may depend on $A_{j}, B_{j}, \Phi$. The terms $\mathcal{I}_{1}(\partial \Omega)$ and $\mathcal{I}_{2}(\partial \Omega)$ are given by

$$
\begin{aligned}
\mathcal{I}_{1}(\partial \Omega)= & \int_{\Omega}\left(A_{1}-A_{2}\right) e^{\mathcal{A}_{1}+\overline{\mathcal{A}_{2}}} \frac{\partial \Phi}{\partial z} \bar{c}\left(a_{2,+} \Lambda_{+}(\tau, \widetilde{x})+a_{2,-} \Lambda_{-}(\tau, \widetilde{x})\right) d x \\
& +\int_{\Omega}\left(A_{1}-A_{2}\right) e^{\mathcal{A}_{1}+\overline{\mathcal{A}_{2}}} \frac{\partial \Phi}{\partial z} a\left(\overline{c_{2,+} \Lambda_{+}(\tau, \widetilde{x})+c_{2,-} \Lambda_{-}(\tau, \widetilde{x})}\right) d x \\
= & -2 \int_{\Omega} \frac{\partial}{\partial \bar{z}} e^{\mathcal{A}_{1}+\overline{\mathcal{A}_{2}}} \frac{\partial \Phi}{\partial z} \bar{c}\left(a_{2,+} \Lambda_{+}(\tau, \widetilde{x})+a_{2,-} \Lambda_{-}(\tau, \widetilde{x})\right) d x \\
& -2 \int_{\Omega} \frac{\partial}{\partial \bar{z}} e^{\mathcal{A}_{1}+\overline{\mathcal{A}_{2}}} \frac{\partial \Phi}{\partial z} a\left(\overline{c_{2,+} \Lambda_{+}(\tau, \widetilde{x})+c_{2,-} \Lambda_{-}(\tau, \widetilde{x})}\right) d x \\
= & -\int_{\partial \Omega}\left(\nu_{1}+i \nu_{2}\right) e^{\mathcal{A}_{1}+\overline{\mathcal{A}_{2}}} \frac{\partial \Phi}{\partial z} \bar{c}\left(a_{2,+} \Lambda_{+}(\tau, \widetilde{x})+a_{2,-} \Lambda_{-}(\tau, \widetilde{x})\right) d \sigma \\
& -\int_{\partial \Omega}\left(\nu_{1}+i \nu_{2}\right) e^{\mathcal{A}_{1}+\overline{\mathcal{A}_{2}}} \frac{\partial \Phi}{\partial z} a\left(\overline{c_{2,+} \Lambda_{+}(\tau, \widetilde{x})+c_{2,-} \Lambda_{-}(\tau, \widetilde{x})}\right) d \sigma
\end{aligned}
$$

and

$$
\begin{aligned}
\mathcal{I}_{2}(\partial \Omega)= & \int_{\Omega}\left(B_{1}-B_{2}\right) e^{\mathcal{B}_{1}+\overline{\mathcal{B}_{2}}} \frac{\partial \bar{\Phi}}{\partial \bar{z}} \bar{b}\left(d_{2,+} \Lambda_{+}(\tau, \widetilde{x})+d_{2,-} \Lambda_{-}(\tau, \widetilde{x})\right) d x \\
& +\int_{\Omega}\left(B_{1}-B_{2}\right) e^{\mathcal{B}_{1}+\overline{\mathcal{B}_{2}}} \frac{\partial \bar{\Phi}}{\partial \bar{z}} d\left(\overline{b_{2,+} \Lambda_{+}(\tau, \widetilde{x})+b_{2,-} \Lambda_{-}(\tau, \widetilde{x})}\right) d x \\
= & -2 \int_{\Omega} \frac{\partial}{\partial z} e^{\mathcal{B}_{1}+\overline{\mathcal{B}_{2}}} \frac{\partial \bar{\Phi}}{\partial \bar{z}} \bar{b}\left(d_{2,+} \Lambda_{+}(\tau, \widetilde{x})+d_{2,-} \Lambda_{-}(\tau, \widetilde{x})\right) d x \\
& -2 \int_{\Omega} \frac{\partial}{\partial z} e^{\mathcal{B}_{1}+\overline{\mathcal{B}_{2}}} \frac{\partial \bar{\Phi}}{\partial \bar{z}} d\left(\overline{b_{2,+} \Lambda_{+}(\tau, \widetilde{x})+b_{2,-} \Lambda_{-}(\tau, \widetilde{x})}\right) d x
\end{aligned}
$$




$$
\begin{aligned}
= & -\int_{\partial \Omega}\left(\nu_{1}-i \nu_{2}\right) e^{\mathcal{B}_{1}+\overline{\mathcal{B}_{2}}} \frac{\partial \bar{\Phi}}{\partial \bar{z}} \bar{b}\left(d_{2,+} \Lambda_{+}(\tau, \widetilde{x})+d_{2,-} \Lambda_{-}(\tau, \widetilde{x})\right) d \sigma \\
& -\int_{\partial \Omega}\left(\nu_{1}-i \nu_{2}\right) e^{\mathcal{B}_{1}+\overline{\mathcal{B}_{2}}} \frac{\partial \bar{\Phi}}{\partial \bar{z}} d\left(\overline{b_{2,+} \Lambda_{+}(\tau, \widetilde{x})+b_{2,-} \Lambda_{+}(\tau, \widetilde{x})}\right) d \sigma .
\end{aligned}
$$

Denote

$$
U_{1}=-e^{\tau \bar{\Phi}} \mathcal{R}_{-\tau, A_{1}}\left\{e_{1} g_{1}\right\}, \quad U_{2}=-e^{\tau \Phi} \widetilde{\mathcal{R}}_{\tau, B_{1}}\left\{e_{1} g_{2}\right\} .
$$

Short calculations give

$$
\begin{aligned}
2 \frac{\partial U_{1}}{\partial \bar{z}} & =\left(-e_{1} g_{1}+A_{1} \mathcal{R}_{-\tau, A_{1}}\left\{e_{1} g_{1}\right\}\right) e^{\tau \bar{\Phi}}, \\
2 \frac{\partial U_{2}}{\partial z} & =\left(-e_{1} g_{2}+B_{1} \widetilde{\mathcal{R}}_{\tau, B_{1}}\left\{e_{1} g_{2}\right\}\right) e^{\tau \Phi} .
\end{aligned}
$$

We have

$$
\begin{aligned}
& \text { (5.15) } \quad \frac{\partial}{\partial z} \mathcal{R}_{-\tau, A_{1}}\left\{e_{1} g_{1}\right\} \\
& =\frac{\partial \mathcal{A}_{1}}{\partial z} \mathcal{R}_{-\tau, A_{1}}\left\{e_{1} g_{1}\right\}+\tau \frac{\partial \Phi}{\partial z} \mathcal{R}_{-\tau, A_{1}}\left\{e_{1} g_{1}\right\}+\mathcal{R}_{-\tau, A_{1}}\left\{\frac{\partial\left(e_{1} g_{1}\right)}{\partial z}\right\} \\
& \quad-\mathcal{R}_{-\tau, A_{1}}\left\{e_{1} g_{1} \frac{\partial \mathcal{A}_{1}}{\partial z}\right\}-\tau \mathcal{R}_{-\tau, A_{1}}\left\{\frac{\partial \Phi}{\partial z} e_{1} g_{1}\right\} \\
& =\mathcal{R}_{-\tau, A_{1}}\left\{\frac{\partial\left(e_{1} g_{1}\right)}{\partial z}\right\} \\
& \quad+\tau \frac{e^{\mathcal{A}_{1}}}{2 \pi} e^{-\tau(\bar{\Phi}-\Phi)} \int_{\Omega} \frac{\frac{\partial \Phi}{\partial \zeta}(\zeta)-\frac{\partial \Phi}{\partial z}(z)}{\zeta-z}\left(e_{1} g_{1} e^{-\mathcal{A}_{1}}\right)\left(\xi_{1}, \xi_{2}\right) e^{\tau(\overline{\Phi(\zeta)}-\Phi(\zeta))} d \xi_{1} d \xi_{2} \\
& +\frac{e^{\mathcal{A}_{1}}}{2 \pi} e^{-\tau(\bar{\Phi}-\Phi)} \int_{\Omega} \frac{\frac{\partial \mathcal{A}_{1}}{\partial \zeta}(\zeta, \bar{\zeta})-\frac{\partial \mathcal{A}_{1}}{\partial z}(z, \bar{z})}{\zeta-z}\left(e_{1} g_{1} e^{-\mathcal{A}_{1}}\right)\left(\xi_{1}, \xi_{2}\right) e^{\tau(\overline{\Phi(\zeta)}-\Phi(\zeta))} d \xi_{1} d \xi_{2} .
\end{aligned}
$$

Let

$$
\begin{aligned}
& \mathfrak{G}(x, g, \mathcal{A}, \tau) \\
& \quad=-\frac{1}{2 \pi} \int_{\Omega} \frac{\left(\tau \frac{\partial \Phi(\zeta)}{\partial \zeta}+\frac{\partial \mathcal{A}(\zeta, \bar{\zeta})}{\partial \zeta}\right)-\left(\tau \frac{\partial \Phi(z)}{\partial z}+\frac{\partial \mathcal{A}(z, \bar{z})}{\partial z}\right)}{\zeta-z} e_{1} g e^{-\mathcal{A}} e^{\tau(\bar{\Phi}-\Phi)} d \xi_{1} d \xi_{2} .
\end{aligned}
$$

We set

$$
\begin{aligned}
& \mathfrak{G}_{1}(x, \tau)=\mathfrak{G}\left(x, g_{1}, \mathcal{A}_{1}, \tau\right), \\
& \mathfrak{G}_{2}(x, \tau)=\overline{\mathfrak{G}\left(x, \bar{g}_{2}, \overline{\mathcal{B}_{1}}, \tau\right)}, \\
& \mathfrak{G}_{3}(x, \tau)=\overline{\mathfrak{G}\left(x, \overline{g_{3}}, \overline{\mathcal{A}_{2}},-\tau\right)}, \quad \mathfrak{G}_{4}(x, \tau)=\mathfrak{G}\left(x, g_{4}, \mathcal{B}_{2},-\tau\right) .
\end{aligned}
$$


By (3.16), (4.6), (5.15) and Proposition 3.5, we obtain

$$
\begin{aligned}
& \frac{\partial}{\partial z} \mathcal{R}_{-\tau, A_{1}}\left\{e_{1} g_{1}\right\} \\
& \quad=\mathcal{R}_{-\tau, A_{1}}\left\{\frac{\partial\left(e_{1} g_{1}\right)}{\partial z}\right\}-e^{\mathcal{A}_{1}} e^{-\tau(\bar{\Phi}-\Phi)} \mathfrak{G}_{1}(\cdot, \tau)+o_{L^{2}(\Omega)}(1 / \tau)
\end{aligned}
$$

Simple computations provide the formula

$$
\begin{aligned}
= & \frac{\partial \mathcal{B}_{1}}{\partial \bar{z}} \widetilde{\mathcal{R}}_{\tau, B_{1}}\left\{e_{1} g_{2}\right\}+\tau \frac{\partial \bar{\Phi}}{\partial \bar{z}} \widetilde{\mathcal{R}}_{\tau, B_{1}}\left\{e_{1} g_{2}\right\}+\widetilde{\mathcal{R}}_{\tau, B_{1}}\left\{\frac{\partial\left(e_{1} g_{2}\right)}{\partial \bar{z}}\right\} \\
& -\tau \widetilde{\mathcal{R}}_{\tau, B_{1}}\left\{\frac{\partial \bar{\Phi}}{\partial \bar{z}} e_{1} g_{2}\right\}-\widetilde{\mathcal{R}}_{\tau, B_{1}}\left\{\frac{\partial \mathcal{B}_{1}}{\partial \bar{z}} e_{1} g_{2}\right\} \\
= & \widetilde{\mathcal{R}}_{\tau, B_{1}}\left\{\frac{\partial\left(e_{1} g_{2}\right)}{\partial \bar{z}}\right\} \\
& +\tau \frac{e^{\mathcal{B}_{1}}}{2 \pi} e^{\tau(\bar{\Phi}-\Phi)} \int_{\Omega} \frac{\frac{\partial \bar{\Phi}}{\partial \bar{\zeta}}(\bar{\zeta})-\frac{\partial \Phi}{\partial \bar{z}}(\bar{z})}{\bar{\zeta}-\bar{z}}\left(e_{1} g_{2} e^{-\mathcal{B}_{1}}\right)\left(\xi_{1}, \xi_{2}\right) e^{\tau(\Phi(\zeta)-\overline{\Phi(\zeta)})} d \xi_{1} d \xi_{2} \\
& +\frac{e^{\mathcal{B}_{1}}}{2 \pi} e^{\tau(\bar{\Phi}-\Phi)} \int_{\Omega} \frac{\frac{\partial \mathcal{B}_{1}}{\partial \bar{\zeta}}(\zeta, \bar{\zeta})-\frac{\partial \mathcal{B}_{1}}{\partial \bar{z}}(z, \bar{z})}{\bar{\zeta}-\bar{z}}\left(e_{1} g_{2} e^{-\mathcal{B}_{1}}\right)\left(\xi_{1}, \xi_{2}\right) e^{\tau(\Phi(\zeta)-\overline{\Phi(\zeta)})} d \xi_{1} d \xi_{2} .
\end{aligned}
$$

By (3.16), (4.6), (5.17) and Proposition 3.5, we have

$$
\frac{\partial}{\partial \bar{z}} \widetilde{\mathcal{R}}_{\tau, B_{1}}\left\{e_{1} g_{2}\right\}=\widetilde{\mathcal{R}}_{\tau, B_{1}}\left\{\frac{\partial\left(e_{1} g_{2}\right)}{\partial \bar{z}}\right\}-e^{\mathcal{B}_{1}} e^{\tau(\bar{\Phi}-\Phi)} \mathfrak{G}_{2}(\cdot, \tau)+o_{L^{2}(\Omega)}(1 / \tau)
$$

Denote

$$
\begin{aligned}
V_{1} & =-e^{-\tau \Phi} \widetilde{\mathcal{R}}_{-\tau,-\overline{A_{2}}}\left\{e_{1} g_{3}\right\}, \\
V_{2} & =-e^{-\tau \bar{\Phi}} \mathcal{R}_{\tau,-\overline{B_{2}}}\left\{e_{1} g_{4}\right\}, \quad \mathcal{P}(x, D)=2\left(A_{1}-A_{2}\right) \frac{\partial}{\partial z}+2\left(B_{1}-B_{2}\right) \frac{\partial}{\partial \bar{z}}, \\
\mathcal{Q}_{+} & =-\left(B_{1}-B_{2}\right) A_{1}-\left(A_{1}-A_{2}\right) B_{2}-2 \frac{\partial}{\partial z}\left(A_{1}-A_{2}\right)+\left(q_{1}-q_{2}\right), \\
\mathcal{Q}_{-} & =-\left(A_{1}-A_{2}\right) B_{1}-\left(B_{1}-B_{2}\right) A_{2}-2 \frac{\partial}{\partial \bar{z}}\left(B_{1}-B_{2}\right)+\left(q_{1}-q_{2}\right) .
\end{aligned}
$$

The following proposition is proved in Section 8.

Proposition 5.2. There exist numbers $\kappa, \kappa_{0}$ independent of $\tau$ such that the following asymptotic formula holds true: 


$$
\begin{aligned}
\left(\mathcal { P } ( x , D ) \left(U_{1}+\right.\right. & \left.\left.U_{2}\right), b_{\tau} e^{\mathcal{B}_{2}-\tau \Phi}+c_{\tau} e^{\mathcal{A}_{2}-\tau \bar{\Phi}}\right)_{L^{2}(\Omega)} \\
& +\left(\mathcal{P}(x, D)\left(a_{\tau} e^{\mathcal{A}_{1}+\tau \Phi}+d_{\tau} e^{\mathcal{B}_{1}+\tau \Phi}\right), V_{1}+V_{2}\right)_{L^{2}(\Omega)} \\
= & +\frac{\kappa_{0}}{\tau}-2 \int_{\partial \Omega}\left(\nu_{1}+i \nu_{2}\right) e^{\mathcal{A}_{1}+\overline{\mathcal{A}_{2}}} \overline{c_{\tau}(\bar{z})} \mathfrak{G}_{1}(x, \tau) d \sigma \\
& +2 \int_{\partial \Omega}\left(\nu_{1}-i \nu_{2}\right) e^{\mathcal{B}_{1}+\overline{\mathcal{B}_{2}}} d_{\tau}(\bar{z}) \overline{\mathfrak{G}_{4}(x, \tau)} d \sigma \\
& +2 \int_{\partial \Omega} a_{\tau}(z) \overline{\mathfrak{G}_{3}(x, \tau)}\left(\nu_{1}+i \nu_{2}\right) e^{\mathcal{A}_{1}+\overline{\mathcal{A}_{2}}} d \sigma \\
& -2 \int_{\partial \Omega}\left(\nu_{1}-i \nu_{2}\right) e^{\mathcal{B}_{1}+\overline{\mathcal{B}_{2}}} \overline{b_{\tau}(z)} \mathfrak{G}_{2}(x, \tau) d \sigma \\
& +\frac{\Lambda_{-}(\tau, \widetilde{x})}{\tau}\left\{\frac{\partial g_{4}(\widetilde{x})}{\partial z}\right\} e^{-\overline{\mathcal{B}_{2}}(\widetilde{x})} \int_{\partial \Omega} \frac{\left(\nu_{1}-i \nu_{2}\right) d e^{\mathcal{B}_{1}+\overline{\mathcal{B}_{2}}}}{\overline{\widetilde{z}}-\bar{z}} d \sigma \\
& -\frac{\Lambda_{-}(\tau, \widetilde{x})}{\tau} \frac{\partial g_{1}(\widetilde{x})}{\partial z} e^{-\mathcal{A}_{1}(\widetilde{x})} \int_{\partial \Omega} \frac{\left(\nu_{1}+i \nu_{2}\right) \bar{c} e^{\mathcal{A}_{1}+\overline{\mathcal{A}_{2}}}}{\widetilde{z}-z} d \sigma \\
& +\frac{\Lambda_{+}(\tau, \widetilde{x})}{\tau} \frac{\overline{\partial g_{3}(\widetilde{x})}}{\partial \bar{z}} e^{-\overline{\mathcal{A}_{2}(\widetilde{x})}} \int_{\partial \Omega} \frac{\left(\nu_{1}+i \nu_{2}\right) a e^{\mathcal{A}_{1}+\overline{\mathcal{A}_{2}}}}{\widetilde{z}-z} d \sigma \\
& -\frac{\Lambda_{+}(\tau, \widetilde{x})}{\tau} \frac{\partial g_{2}(\widetilde{x})}{\partial \bar{z}} e^{-\mathcal{B}_{1}(\widetilde{x})} \int_{\partial \Omega} \frac{\left(\nu_{1}-i \nu_{2}\right) \bar{b} e^{\mathcal{B}_{1}+\overline{\mathcal{B}_{2}}}}{\overline{\widetilde{z}}-\bar{z}} d \sigma \\
& -\frac{2 \pi}{\tau}\left(\mathcal{Q}_{+} a \bar{b} \Lambda_{+}(\tau, \widetilde{x}) e^{\mathcal{A}_{1}+\overline{\mathcal{B}_{2}}}+\mathcal{Q}_{-} \bar{c} d \Lambda_{-}(\tau, \widetilde{x}) e^{\mathcal{B}_{1}+\overline{\mathcal{A}_{2}}}\right)(\widetilde{x}) .
\end{aligned}
$$

By (5.13), (5.14), (5.16), (5.18) and Proposition 3.3, there exists a constant $\mathcal{C}_{0}$ independent of $\tau$ such that

$$
\begin{aligned}
& \left.\mathcal{P}(x, D)\left(U_{1}+U_{2}\right), V_{1}+V_{2}\right)_{L^{2}(\Omega)} \\
= & \left(( A _ { 1 } - A _ { 2 } ) \left(-2\left(\mathcal{R}_{-\tau, A_{1}}\left\{\frac{\partial\left(e_{1} g_{1}\right)}{\partial z}\right\}-e^{\mathcal{A}_{1}} e^{-\tau(\bar{\Phi}-\Phi)} \mathfrak{G}_{1}+o_{L^{2}(\Omega)}(1 / \tau)\right) e^{\tau \bar{\Phi}}\right.\right. \\
& \left.\left.+\left(-e_{1} g_{2}+\frac{B_{1} e_{1} g_{2}}{2 \tau \partial_{z} \Phi}+o_{L^{2}(\Omega)}(1 / \tau)\right) e^{\tau \Phi}\right), V_{1}+V_{2}\right)_{L^{2}(\Omega)} \\
& +\left(\left(B_{1}-B_{2}\right)\left(-e_{1} g_{1}+A_{1} \frac{e_{1} g_{1}}{2 \tau \overline{\partial_{z} \Phi}}+o_{L^{2}(\Omega)}(1 / \tau)\right) e^{\tau \bar{\Phi}}, V_{1}+V_{2}\right)_{L^{2}(\Omega)} \\
- & \left(2\left(B_{1}-B_{2}\right)\left(\widetilde{\mathcal{R}}_{\tau, B_{1}}\left\{\frac{\partial\left(e_{1} g_{2}\right)}{\partial \bar{z}}\right\}-e^{\mathcal{B}_{1}} e^{\tau(\bar{\Phi}-\Phi)} \mathfrak{G}_{2}+o_{L^{2}(\Omega)}(1 / \tau)\right) e^{\tau \Phi}, V_{1}+V_{2}\right)_{L^{2}(\Omega)} \\
= & \mathcal{C}_{0} / \tau+o(1 / \tau) \quad \text { as }|\tau| \rightarrow+\infty .
\end{aligned}
$$

Next we claim that

$$
\begin{aligned}
& \left(\mathcal{P}(x, D)\left(u_{0} e^{\tau \varphi}\right), v\right)_{L^{2}(\Omega)}=o(1 / \tau) \quad \text { as }|\tau| \rightarrow+\infty, \\
& \left(\mathcal{P}(x, D) u_{1}, v_{0} e^{-\tau \varphi}\right)_{L^{2}(\Omega)}=o(1 / \tau) \quad \text { as }|\tau| \rightarrow+\infty .
\end{aligned}
$$


Let us first prove (5.21). By (4.27), (4.57), (5.16), (5.18), and Propositions 3.2 and 3.4 , we have

$$
\begin{aligned}
& \left(\mathcal{P}(x, D) u_{1}, v_{0} e^{-\tau \varphi}\right)_{L^{2}(\Omega)} \\
& \quad=\left(\mathcal{P}(x, D) \mathcal{U}, v_{0} e^{-\tau \varphi}\right)_{L^{2}(\Omega)}+o(1 / \tau) \quad \text { as }|\tau| \rightarrow+\infty
\end{aligned}
$$

We recall that the functions $\mathcal{U}$ and $\mathcal{V}$ are defined by (5.8). By (4.57) we obtain from (5.22)

$$
\begin{aligned}
& \left(\mathcal{P}(x, D) u_{1}, v_{0} e^{-\tau \varphi}\right) L^{2}(\Omega) \\
= & \tau \int_{\Omega} 2 \chi\left(\frac{\partial \Phi}{\partial z}\left(A_{1}-A_{2}\right) a e^{\mathcal{A}_{1}+i \tau \psi}+\frac{\partial \bar{\Phi}}{\partial \bar{z}}\left(B_{1}-B_{2}\right) b e^{\mathcal{B}_{1}-i \tau \psi}\right) \overline{v_{0}} d x+o(1 / \tau)
\end{aligned}
$$

as $|\tau| \rightarrow+\infty$. Here $\chi \in C_{0}^{\infty}(\bar{\Omega})$ is a function such that $\chi \equiv 1$ in some neighborhood of $\operatorname{supp} e_{2}$ and $\mathcal{H} \backslash \partial \Omega \subset \operatorname{supp} e_{2}$.

By (4.53), the functions $v_{0,+}=e^{-i \tau \psi} \overline{v_{0}}$ and $v_{0,-}=e^{i \tau \psi} \overline{v_{0}}$ satisfy

$$
e^{\tau \Phi} \overline{L_{2}(x, D) *\left(e^{-\tau \bar{\Phi}} v_{0,+}\right)}=\bar{h}_{2} e^{i \tau \psi} \quad \text { and } \quad e^{\tau \bar{\Phi}} \overline{L_{2}(x, D)^{*}\left(e^{-\tau \Phi} v_{0,-}\right)}=\bar{h}_{2} e^{-i \tau \psi}
$$

More explicitly, there exist first-order operators $\mathcal{P}_{k}(x, D), k=1,2$, such that

$$
\begin{aligned}
& e^{\tau \Phi} \overline{L_{2}(x, D)^{*}\left(e^{-\tau \bar{\Phi}} v_{0,+}\right)}=\Delta \overline{v_{0,+}}-2 \tau \frac{\partial \Phi}{\partial z}\left(2 \frac{\partial \overline{v_{0,+}}}{\partial \bar{z}}-A_{2} \bar{v}_{0,+}\right)+\mathcal{P}_{1}(x, D) \overline{v_{0,+}} \\
& =o_{L^{2}(\Omega)}(1 / \tau) \quad \text { as }|\tau| \rightarrow+\infty, \\
& e^{\tau \bar{\Phi}} \overline{L_{2}(x, D) *\left(e^{-\tau \Phi} v_{0,-}\right)}=\Delta \overline{v_{0,-}}-2 \tau \frac{\partial \bar{\Phi}}{\partial \bar{z}}\left(2 \frac{\partial \overline{v_{0,-}}}{\partial z}-B_{2} \bar{v}_{0,-}\right)+\mathcal{P}_{2}(x, D) \overline{v_{0,-}} \\
& =o_{L^{2}(\Omega)}(1 / \tau) \quad \text { as }|\tau| \rightarrow+\infty .
\end{aligned}
$$

In the above equalities we used (4.55).

Let $\chi_{1} \in C_{0}^{\infty}(\bar{\Omega})$ be a function such that $\chi_{1} \equiv 1$ on supp $\chi$ and $g \in C^{2}(\bar{\Omega})$.

Taking the scalar product of the first equation with $\overline{\chi_{1} g}$, we obtain

$$
\begin{aligned}
\int_{\Omega} 2 \tau & \frac{\partial \Phi}{\partial z} \overline{v_{0,+}} \chi_{1}\left(2 \frac{\partial}{\partial \bar{z}}+A_{2}\right) g d x \\
& =o(1 / \tau)-\int_{\Omega}\left(\overline{v_{0,+}}\left(\Delta+\mathcal{P}_{1}(x, D)^{*}\right)\left(\chi_{1} g\right)+2 \tau \overline{v_{0,+}} \frac{\partial \Phi}{\partial z} g\left(\frac{\partial}{\partial \bar{z}}+A_{2}\right) \chi_{1}\right) d x
\end{aligned}
$$

By (4.57) we have

$$
\int_{\Omega} \tau \frac{\partial \Phi}{\partial z} \overline{v_{0,+}} \chi_{1}\left(2 \frac{\partial}{\partial \bar{z}}+A_{2}\right) g d x=o(1 / \tau) \quad \text { as }|\tau| \rightarrow+\infty .
$$


Taking the scalar product of the second equation with $\overline{\chi_{1} g}$ with $g \in C^{2}(\bar{\Omega})$, we have

$$
\begin{aligned}
\int_{\Omega} 2 \tau & \frac{\partial \Phi}{\partial \bar{z}} \overline{v_{0,-}} \chi_{1}\left(2 \frac{\partial}{\partial z}+B_{2}\right) g d x \\
& =o(1 / \tau)-\int_{\Omega}\left(\overline{v_{0,-}}\left(\Delta+\mathcal{P}_{2}(x, D)^{*}\right)\left(\chi_{1} g\right)+2 \tau \overline{v_{0,-}} g \frac{\partial \Phi}{\partial \bar{z}}\left(2 \frac{\partial}{\partial z}+B_{2}\right) \chi_{1}\right) d x
\end{aligned}
$$

By (4.57) we obtain

$$
\int_{\Omega} 2 \tau \frac{\partial \Phi}{\partial \bar{z}} \overline{v_{0,-}} \chi_{1}\left(2 \frac{\partial}{\partial z}+B_{2}\right) g d x=o(1 / \tau) \quad \text { as }|\tau| \rightarrow+\infty .
$$

Taking $g$ such that $\left(2 \frac{\partial}{\partial \bar{z}}+A_{2}\right) g=\left(A_{1}-A_{2}\right) e^{\mathcal{A}_{1}} a(z)$ in (5.24) and $g$ such that $\left(2 \frac{\partial}{\partial z}+B_{2}\right) g=\left(B_{1}-B_{2}\right) b(\bar{z}) e^{\mathcal{B}_{1}}$ in (5.25), from (5.22) we obtain (5.21).

In order to prove (5.20), we observe that

$$
\begin{aligned}
\left(\mathcal{P}(x, D)\left(u_{0} e^{\tau \varphi}\right)\right. & , v)_{L^{2}(\Omega)}=\left(\mathcal{P}(x, D)\left(u_{0} e^{\tau \varphi}\right), \mathcal{V}\right)_{L^{2}(\Omega)}+o(1 / \tau) \\
& =\left(\mathcal{P}(x, D)\left(u_{0} e^{\tau \varphi}\right), \chi \mathcal{V}\right)_{L^{2}(\Omega)}+o(1 / \tau) \\
& =\left(u_{0} e^{\tau \varphi}, \mathcal{P}(x, D)^{*}(\chi \mathcal{V})\right)_{L^{2}(\Omega)}+o(1 / \tau) \quad \text { as }|\tau| \rightarrow+\infty
\end{aligned}
$$

Then we can finish the proof of (5.20) using arguments similar to (5.23)-(5.24).

Denote

$$
\begin{array}{ll}
\mathcal{M}_{1}=\frac{e_{2} g_{5}}{2 \tau^{2} \partial_{z} \Phi}+\widetilde{\mathcal{R}}_{\tau, B_{1}}\left\{e_{1} g_{5} / \tau\right\}, & \mathcal{M}_{2}=\frac{e_{2} g_{6}}{2 \tau^{2} \overline{\partial_{z} \Phi}}+\widetilde{\mathcal{R}}_{-\tau, A_{1}}\left\{e_{1} g_{6} / \tau\right\}, \\
\mathcal{M}_{3}=\frac{e_{2} g_{7}}{2 \tau^{2} \partial_{z} \Phi}-\frac{1}{\tau} \mathcal{R}_{\tau,-\overline{A_{2}}}\left\{e_{1} g_{7}\right\}, & \mathcal{M}_{4}=\frac{e_{2} g_{8}}{\tau^{2} \partial_{\bar{z}} \bar{\Phi}}-\widetilde{\mathcal{R}}_{-\tau,-\overline{B_{2}}}\left\{e_{1} g_{8} / \tau\right\} .
\end{array}
$$

Then there exists a constant $\mathcal{C}$ independent of $\tau$ such that

$$
\begin{aligned}
& \left(\mathcal{P}(x, D)\left(e^{\tau \Phi} \mathcal{M}_{1}+e^{\tau \bar{\Phi}} \mathcal{M}_{2}\right), v\right)_{L^{2}(\Omega)} \\
+ & \left(\mathcal{P}(x, D) u, e^{-\tau \Phi} \mathcal{M}_{3}+e^{-\tau \bar{\Phi}} \mathcal{M}_{4}\right)_{L^{2}(\Omega)}=\mathcal{C} / \tau+o(1 / \tau) \quad \text { as }|\tau| \rightarrow+\infty
\end{aligned}
$$

Denote

$$
\begin{aligned}
& \mathcal{X}_{1}=-\frac{e_{2}\left(g_{1}+\widehat{g}_{1} / \tau\right)}{2 \tau \partial_{\bar{z}} \bar{\Phi}}-\mathcal{R}_{-\tau, A_{1}}\left\{e_{1} \widehat{g}_{1} / \tau\right\}, \mathcal{X}_{2}=-\frac{e_{2}\left(g_{2}+\widehat{g}_{2} / \tau\right)}{2 \tau \partial_{z} \Phi}-\widetilde{\mathcal{R}}_{\tau, B_{1}}\left\{e_{1} \widehat{g}_{2} / \tau\right\} \\
& \mathcal{X}_{3}=\frac{e_{2}\left(g_{3}+\widehat{g}_{3} / \tau\right)}{2 \tau \partial_{z} \Phi}-\widetilde{\mathcal{R}}_{-\tau,-\overline{A_{2}}}\left\{e_{1} \widehat{g}_{3} / \tau\right\}, \mathcal{X}_{4}=\frac{e_{2}\left(g_{4}+\widehat{g}_{4} / \tau\right)}{2 \tau \partial_{\bar{z}} \bar{\Phi}}-\mathcal{R}_{\tau,-\overline{B_{2}}}\left\{e_{1} \widehat{g}_{4} / \tau\right\}
\end{aligned}
$$

Then, using the stationary phase argument, we conclude that 


$$
\begin{aligned}
= & \mathcal{C}_{1}+\mathcal{C}_{2} / \tau \\
& +\frac{1}{\tau} \int_{\widetilde{\Gamma}}\left(\left(A_{1}-A_{2}\right) \frac{\partial \Phi}{\partial z} \mathcal{X}_{2} \bar{b} e^{\overline{\mathcal{B}_{2}}} e^{2 \tau i \psi}-\left(B_{1}-B_{2}\right) \frac{\partial \bar{\Phi}}{\partial \bar{z}} \mathcal{X}_{1} b e^{\overline{\mathcal{A}_{2}}} e^{-2 \tau i \psi}\right) \frac{(\nabla \psi, \nu)}{2 i|\nabla \psi|^{2}} d \sigma \\
& +\frac{1}{\tau} \int_{\widetilde{\Gamma}}\left(\left(A_{1}-A_{2}\right) \frac{\partial \Phi}{\partial z} \overline{\mathcal{X}_{3}} a e^{\mathcal{A}_{1}} e^{2 \tau i \psi}-\left(B_{1}-B_{2}\right) \frac{\partial \bar{\Phi}}{\partial \bar{z}} \overline{\mathcal{X}_{4}} \bar{a} e^{\mathcal{B}_{1}} e^{-2 \tau i \psi}\right) \frac{(\nabla \psi, \nu)}{2 i|\nabla \psi|^{2}} d \sigma \\
& +o(1 / \tau) \quad \text { as }|\tau| \rightarrow+\infty .
\end{aligned}
$$

Here the constants $\mathcal{C}_{1}$ and $\mathcal{C}_{2}$ are independent of $\tau$.

Proposition 5.3. Under the conditions of Theorem 1.3, we have

$$
A_{1}=A_{2}, \quad B_{1}=B_{2} \quad \text { on } \widetilde{\Gamma} .
$$

Moreover, for any function $\Phi$ satisfying (2.1) and (2.2) and for any functions $a, b, c, d$ satisfying (4.3), (4.4), (4.31) and (4.32), we have

$$
\begin{gathered}
\Im(\Phi, a, b, c, d) \\
:=\int_{\widetilde{\Gamma}}\left\{\left(\nu_{1}+i \nu_{2}\right) \frac{\partial \Phi}{\partial z} a(z) \overline{c(\bar{z})} e^{\mathcal{A}_{1}+\overline{\mathcal{A}_{2}}}+\left(\nu_{1}-i \nu_{2}\right) \frac{\partial \bar{\Phi}}{\partial \bar{z}} d(\bar{z}) \overline{b(z)} e^{\mathcal{B}_{1}+\overline{\mathcal{B}_{2}}}\right\} d \sigma=0 .
\end{gathered}
$$

Proof. Let $\widehat{x} \in \operatorname{Int} \widetilde{\Gamma}$ and $\Gamma_{*}$ be an arc containing $\widehat{x}$ such that $\Gamma_{*} \subset \subset \widetilde{\Gamma}$. By Proposition 2.2 there exists a weight function $\Phi$ satisfying (2.4) and (2.6). Then the boundary integrals in (5.9) and (5.10) have the following asymptotics:

$$
\begin{aligned}
\int_{\widetilde{\Gamma}}\left(B_{1}-B_{2}\right) d_{\tau}(\bar{z}) \overline{c_{\tau}(\bar{z})} e^{\mathcal{B}_{1}+\overline{\mathcal{A}_{2}}-2 i \tau \psi} d \sigma & +\int_{\widetilde{\Gamma}}\left(A_{1}-A_{2}\right)\left(\nu_{1}-i \nu_{2}\right) a_{\tau}(z) \overline{b_{\tau}(z)} e^{\mathcal{A}_{1}+\overline{\mathcal{B}_{2}}+2 i \tau \psi} d \sigma \\
= & \sum_{x \in \mathcal{G} \backslash\left\{x_{-}, x_{+}\right\}}\left\{\left(\frac{2 \pi}{i \frac{\partial^{2} \psi}{\partial \bar{\tau}^{2}}(x)}\right)^{1 / 2}\left(\bar{c} d\left(B_{1}-B_{2}\right)\right)(x) \frac{e^{\left(\mathcal{B}_{1}+\overline{\mathcal{A}_{2}}-2 \tau i \psi\right)(x)}}{\sqrt{\tau}}\right. \\
& \left.+\left(\frac{2 \pi}{-i \frac{\partial^{2} \psi}{\partial \bar{\tau}^{2}}(x)}\right)^{1 / 2}\left(a \bar{b}\left(A_{1}-A_{2}\right)\right)(x) \frac{e^{\left(\mathcal{A}_{1}+\overline{\mathcal{B}_{2}}+2 \tau i \psi\right)(x)}}{\sqrt{\tau}}\right\} \\
& +O(1 / \tau) \text { as }|\tau| \rightarrow+\infty .
\end{aligned}
$$

We recall that the set $\mathcal{G}$ is introduced in (2.4). Moreover, in order to avoid the contribution from the points $x_{ \pm}$, functions $a, b$ are chosen in such a way that 


$$
\begin{aligned}
\frac{\partial^{|\beta|} a}{\partial x_{1}^{\beta_{1}} \partial x_{2}^{\beta_{2}}}\left(x_{ \pm}\right) & =\frac{\partial^{|\beta|} b}{\partial x_{1}^{\beta_{1}} \partial x_{2}^{\beta_{2}}}\left(x_{ \pm}\right)=\frac{\partial^{|\beta|} c}{\partial x_{1}^{\beta_{1}} \partial x_{2}^{\beta_{2}}}\left(x_{ \pm}\right) \\
& =\frac{\partial^{|\beta|} d}{\partial x_{1}^{\beta_{1}} \partial x_{2}^{\beta_{2}}}\left(x_{ \pm}\right)=0 \quad \forall|\beta| \in\{0, \ldots, 5\} .
\end{aligned}
$$

Let $\widetilde{\chi}_{1} \in C^{\infty}(\partial \Omega)$ be equal to 1 near $x_{ \pm}$and have support in a small neighborhood of those points. Then

$$
\begin{aligned}
\int_{\Gamma_{*}} \tilde{\chi}_{1}\left(B_{1}-B_{2}\right) & d_{\tau} \overline{\bar{c}_{\tau}} e^{\mathcal{B}_{1}+\overline{\mathcal{A}_{2}}-2 i \tau \psi} d \sigma+\int_{\Gamma_{*}} \tilde{\chi}_{1}\left(A_{1}-A_{2}\right)\left(\nu_{1}-i \nu_{2}\right) a_{\tau} \overline{b_{\tau}} e^{\mathcal{A}_{1}+\overline{\mathcal{B}_{2}}+2 i \tau \psi} d \sigma \\
= & \int_{\Gamma_{*}} \frac{\tilde{\chi}_{1}\left(B_{1}-B_{2}\right) d_{\tau} \overline{c_{\tau}} e^{\mathcal{B}_{1}+\overline{\mathcal{A}_{2}}}}{-2 i \tau \frac{\partial \psi}{\partial \bar{\tau}}} \frac{\partial e^{-2 i \tau \psi}}{\partial \vec{\tau}} d \sigma \\
& +\int_{\Gamma_{*}} \frac{\widetilde{\chi}_{1}\left(A_{1}-A_{2}\right)\left(\nu_{1}-i \nu_{2}\right) a_{\tau} \overline{b_{\tau}} e^{\mathcal{A}_{1}+\overline{\mathcal{B}_{2}}}}{2 i \tau \frac{\partial \psi}{\partial \bar{\tau}}} \frac{\partial e^{2 i \tau \psi}}{\partial \vec{\tau}} d \sigma \\
= & \int_{\Gamma_{*}} \frac{\partial}{\partial \vec{\tau}}\left(\frac{\widetilde{\chi}_{1}\left(B_{1}-B_{2}\right) d_{\tau} \overline{\bar{c}_{\tau}} e^{\mathcal{B}_{1}+\overline{\mathcal{A}_{2}}}}{2 i \tau \frac{\partial \psi}{\partial \vec{\tau}}}\right) e^{-2 i \tau \psi} d \sigma \\
& -\int_{\Gamma_{*}} \frac{\partial}{\partial \vec{\tau}}\left(\frac{\tilde{\chi}_{1}\left(A_{1}-A_{2}\right)\left(\nu_{1}-i \nu_{2}\right) a_{\tau} \overline{b_{\tau}} e^{\mathcal{A}_{1}+\overline{\mathcal{B}_{2}}}}{2 i \tau \frac{\partial \psi}{\partial \bar{\tau}}}\right) e^{2 i \tau \psi} d \sigma=O(1 / \tau) .
\end{aligned}
$$

In order to obtain the last estimate, we used that by (5.32) and (2.7) the functions

$$
\frac{\partial}{\partial \vec{\tau}}\left(\frac{\tilde{\chi}_{1}\left(B_{1}-B_{2}\right) d_{\tau} \overline{\bar{c}_{\tau}} e^{\mathcal{B}_{1}+\overline{\mathcal{A}_{2}}}}{2 i \frac{\partial \psi}{\partial \bar{\tau}}}\right), \quad \frac{\partial}{\partial \vec{\tau}}\left(\frac{\tilde{\chi}_{1}\left(A_{1}-A_{2}\right)\left(\nu_{1}-i \nu_{2}\right) a_{\tau} \overline{b_{\tau}} e^{\mathcal{A}_{1}+\overline{\mathcal{B}_{2}}}}{2 i \frac{\partial \psi}{\partial \bar{\tau}}}\right)
$$

are bounded. By (5.5), (5.9)-(5.12), (5.19)-(5.21), (5.27)-(5.28) and (5.31), we can represent the right-hand side of (5.4) as

$$
\begin{array}{r}
O(1 / \tau)=\tau F_{1}+F_{0}+\sum_{x \in \mathcal{G} \backslash\left\{x_{-}, x_{+}\right\}}\left(\left(\frac{2 \pi}{i \frac{\partial^{2} \psi}{\partial \bar{\tau}^{2}}(x)}\right)^{1 / 2}\left(\bar{c} d\left(B_{1}-B_{2}\right)\right)(x) \frac{e^{\left(\mathcal{B}_{1}+\overline{\mathcal{A}_{2}}-2 \tau i \psi\right)(x)}}{\sqrt{\tau}}\right. \\
\left.+\left(\frac{2 \pi}{-i \frac{\partial^{2} \psi}{\partial \bar{\tau}^{2}}(x)}\right)^{1 / 2}\left(a \bar{b}\left(A_{1}-A_{2}\right)\right)(x) \frac{e^{\left(\mathcal{B}_{1}+\overline{\mathcal{A}_{2}}+2 \tau i \psi\right)(x)}}{\sqrt{\tau}}\right) .
\end{array}
$$

Taking into account that $F_{1}$ is equal to the left-hand side of (5.30), we obtain the equality (5.30). Using (2.6) and applying Bohr's theorem (see, e.g., [5, p. 393]), we obtain (5.29).

Thanks to (5.5), (5.9)-(5.12), (5.19)-(5.21), (5.27)-(5.29) and (5.31), we can write the right-hand side of (5.4) as 


$$
I_{0}+I_{1}+I_{2}=\sum_{k=1}^{3} \tau^{2-k}\left(\kappa_{k}+\widetilde{\kappa}_{k}\right)+\kappa
$$

$+\int_{\Gamma_{0}}\left(A_{1}-A_{2}\right)\left(\nu_{1}-i \nu_{2}\right) a_{\tau} \overline{b_{\tau}} e^{\mathcal{A}_{1}+\overline{\mathcal{B}_{2}}} d \sigma+\int_{\Gamma_{0}}\left(B_{1}-B_{2}\right)\left(\nu_{1}+i \nu_{2}\right) d_{\tau} \overline{c_{\tau}} e^{\mathcal{B}_{1}+\overline{\mathcal{A}_{2}}} d \sigma$

$-\frac{1}{2 \tau i} \int_{\Gamma_{0}} \mathcal{Q}_{+} \bar{a} b e^{\mathcal{B}_{1}+\overline{\mathcal{A}_{2}}} \frac{(\nabla \psi, \nu)}{|\nabla \psi|^{2}} d \sigma-\frac{1}{2 \tau i} \int_{\Gamma_{0}} \mathcal{Q}_{-} a \bar{b} e^{\mathcal{A}_{1}+\overline{\mathcal{B}_{2}}} \frac{(\nabla \psi, \nu)}{|\nabla \psi|^{2}} d \sigma$

$-\frac{\pi}{\tau}\left(\left(\mathcal{Q}_{+} a \bar{b}\right)(\widetilde{x}) \Lambda_{+}(\tau, \widetilde{x}) e^{\left(\mathcal{A}_{1}+\overline{\mathcal{B}_{2}}\right)(\widetilde{x})}+\left(\mathcal{Q}_{-} d \bar{c}\right)(\widetilde{x}) \Lambda_{-}(\tau, \widetilde{x}) e^{\left(\mathcal{B}_{1}+\overline{\mathcal{A}_{2}}\right)(\widetilde{x})}\right)$

$+\frac{1}{\tau}\left(\mathcal{I}_{1}(\partial \Omega)+\mathcal{I}_{2}(\partial \Omega)\right)-2 \int_{\partial \Omega}\left(\nu_{1}+i \nu_{2}\right) e^{\mathcal{A}_{1}+\overline{\mathcal{A}_{2}}} \overline{c_{\tau}(\bar{z})} \mathfrak{G}_{1}(x, \tau) d \sigma$

$+2 \int_{\partial \Omega}\left(\nu_{1}-i \nu_{2}\right) e^{\mathcal{B}_{1}+\overline{\mathcal{B}_{2}}} d_{\tau}(\bar{z}) \overline{\mathfrak{G}_{4}(x, \tau)} d \sigma$

$+2 \int_{\partial \Omega}\left(\nu_{1}+i \nu_{2}\right) e^{\mathcal{A}_{1}+\overline{\mathcal{A}_{2}}} a_{\tau}(z) \overline{\mathfrak{G}_{3}(x, \tau)} d \sigma$

$-2 \int_{\partial \Omega}\left(\nu_{1}-i \nu_{2}\right) e^{\mathcal{B}_{1}+\overline{\mathcal{B}_{2}}} \overline{b_{\tau}(z)} \mathfrak{G}_{2}(x, \tau) d \sigma$

$+\frac{\Lambda_{-}(\tau, \widetilde{x})}{\tau} \frac{\overline{\partial g_{4}(\widetilde{x})}}{\partial z} e^{-\overline{\mathcal{B}_{2}}(\widetilde{x})} \int_{\partial \Omega} \frac{\left(\nu_{1}-i \nu_{2}\right) d e^{\mathcal{B}_{1}+\overline{\mathcal{B}_{2}}}}{\overline{\widetilde{z}}-\bar{z}} d \sigma$

$-\frac{\Lambda_{-}(\tau, \widetilde{x})}{\tau} \frac{\partial g_{1}(\widetilde{x})}{\partial z} e^{-\mathcal{A}_{1}(\widetilde{x})} \int_{\partial \Omega} \frac{\left(\nu_{1}+i \nu_{2}\right) \bar{c} e^{\mathcal{A}_{1}+\overline{\mathcal{A}_{2}}}}{\widetilde{z}-z} d \sigma$

$+\frac{\Lambda_{+}(\tau, \widetilde{x})}{\tau} \frac{\overline{\partial g_{3}(\widetilde{x})}}{\partial \bar{z}} e^{-\overline{\mathcal{A}_{2}}(\widetilde{x})} \int_{\partial \Omega} \frac{\left(\nu_{1}+i \nu_{2}\right) a e^{\mathcal{A}_{1}+\overline{\mathcal{A}_{2}}}}{\widetilde{z}-z} d \sigma$

$-\frac{\Lambda_{+}(\tau, \widetilde{x})}{\tau} \frac{\partial g_{2}(\widetilde{x})}{\partial \bar{z}} e^{-\mathcal{B}_{1}(\widetilde{x})} \int_{\partial \Omega} \frac{\left(\nu_{1}-i \nu_{2}\right) \bar{b} e^{\mathcal{B}_{1}+\overline{\mathcal{B}_{2}}}}{\overline{\widetilde{z}}-\bar{z}} d \sigma+o(1 / \tau) \quad$ as $|\tau| \rightarrow+\infty$.

We note that $\kappa_{k}$ and $\widetilde{\kappa}_{k}$ denote generic constants which are independent of $\tau$. In order to transform some terms in the above equality, we need the following proposition:

Proposition 5.4. There exist a holomorphic function $\Theta \in H^{1 / 2}(\Omega)$ and an antiholomorphic function $\widetilde{\Theta} \in H^{1 / 2}(\Omega)$ such that

$$
\begin{aligned}
& \left.\Theta\right|_{\widetilde{\Gamma}}=e^{\mathcal{A}_{1}+\overline{\mathcal{A}_{2}}},\left.\quad \widetilde{\Theta}\right|_{\widetilde{\Gamma}}=e^{\mathcal{B}_{1}+\overline{\mathcal{B}_{2}}}, \\
& e^{\mathcal{B}_{1}+\overline{\mathcal{B}_{2}}} \Theta-e^{\overline{\mathcal{A}_{1}}+\overline{\mathcal{A}_{2}}} \widetilde{\Theta}=0 \quad \text { on } \Gamma_{0} .
\end{aligned}
$$

Proof. Consider the extremal problem

$$
J(\Psi, \widetilde{\Psi})=\left\|e^{\mathcal{A}_{1}+\overline{\mathcal{A}_{2}}} \frac{\partial \Phi}{\partial z} a \bar{c}-\Psi\right\|_{L^{2}(\widetilde{\Gamma})}^{2}+\left\|e^{\mathcal{B}_{1}+\overline{\mathcal{B}_{2}}} \frac{\partial \bar{\Phi}}{\partial \bar{z}} \bar{b}-\widetilde{\Psi}\right\|_{L^{2}(\widetilde{\Gamma})}^{2} \rightarrow \inf
$$


(5.37) $\frac{\partial \Psi}{\partial \bar{z}}=0 \quad$ in $\Omega, \quad \frac{\partial \widetilde{\Psi}}{\partial z}=0 \quad$ in $\Omega,\left.\quad\left(\left(\nu_{1}+i \nu_{2}\right) \Psi+\left(\nu_{1}-i \nu_{2}\right) \widetilde{\Psi}\right)\right|_{\Gamma_{0}}=0$.

Here the functions $a, b, c, d$ satisfy (4.3), (4.4), (4.31) and (4.32). Denote the unique solution to this extremal problem $(5.36),(5.37)$ by $(\widehat{\Psi}, \widehat{\widetilde{\Psi}})$. Applying Lagrange's principle, we obtain

$$
\operatorname{Re}\left(e^{\mathcal{A}_{1}+\overline{\mathcal{A}_{2}}} \frac{\partial \Phi}{\partial z} a \bar{c}-\widehat{\Psi}, \delta\right)_{L^{2}(\widetilde{\Gamma})}+\operatorname{Re}\left(e^{\mathcal{B}_{1}+\overline{\mathcal{B}_{2}}} \frac{\partial \bar{\Phi}}{\partial \bar{z}} \bar{b} d-\widehat{\widetilde{\Psi}}, \widetilde{\delta}\right)_{L^{2}(\widetilde{\Gamma})}=0
$$

for any $\delta, \widetilde{\delta}$ from $H^{1 / 2}(\Omega)$ such that

$$
\frac{\partial \delta}{\partial \bar{z}}=0 \quad \text { in } \Omega, \quad \frac{\partial \widetilde{\delta}}{\partial z}=0 \quad \text { in } \Omega,\left.\quad\left(\nu_{1}+i \nu_{2}\right) \delta\right|_{\Gamma_{0}}=-\left.\left(\nu_{1}-i \nu_{2}\right) \widetilde{\delta}\right|_{\Gamma_{0}}
$$

and there exist $P, \widetilde{P} \in H^{1 / 2}(\Omega)$ such that

$$
\begin{aligned}
& \frac{\partial P}{\partial \bar{z}}=0 \quad \text { in } \Omega, \quad \frac{\partial \widetilde{P}}{\partial z}=0 \quad \text { in } \Omega, \\
& \left(\nu_{1}+i \nu_{2}\right) P=\overline{e^{\mathcal{A}_{1}+\overline{\mathcal{A}_{2}}} \frac{\partial \Phi}{\partial z} a \bar{c}-\widehat{\Psi}} \text { on } \widetilde{\Gamma}, \\
& \left(\nu_{1}-i \nu_{2}\right) \widetilde{P}=e^{\overline{\mathcal{B}_{1}+\overline{\mathcal{B}_{2}}} \frac{\partial \bar{\Phi}}{\partial \bar{b}} \bar{b}-\widehat{\widetilde{\Psi}}} \text { on } \widetilde{\Gamma}, \\
& \left.(P-\widetilde{P})\right|_{\Gamma_{0}}=0 .
\end{aligned}
$$

Denote $\Psi_{0}(z)=\frac{1}{2 i}(P(z)-\overline{\widetilde{P}(\bar{z})})$ and $\Phi_{0}(z)=\frac{1}{2}(P(z)+\overline{\widetilde{P}(\bar{z})})$. Equality (5.41) yields

$$
\left.\operatorname{Im} \Psi_{0}\right|_{\Gamma_{0}}=\left.\operatorname{Im} \Phi_{0}\right|_{\Gamma_{0}}=0 .
$$

Note that

$$
P=\Phi_{0}+i \Psi_{0}, \quad \overline{\widetilde{P}}=\Phi_{0}-i \Psi_{0} .
$$

From (5.38), taking $\delta=\widehat{\Psi}$ and $\widetilde{\delta}=\widehat{\widetilde{\Psi}}$, we have

$$
\operatorname{Re}\left(e^{\mathcal{A}_{1}+\overline{\mathcal{A}_{2}}} \frac{\partial \Phi}{\partial z} a \bar{c}-\widehat{\Psi}, \widehat{\Psi}\right)_{L^{2}(\widetilde{\Gamma})}+\operatorname{Re}\left(e^{\mathcal{B}_{1}+\overline{\mathcal{B}_{2}}} \frac{\partial \bar{\Phi}}{\partial \bar{b}} \bar{b} d-\widehat{\widetilde{\Psi}}, \widehat{\widetilde{\Psi}}\right)_{L^{2}(\widetilde{\Gamma})}=0
$$

By (5.39), (5.40) and (5.43), we have

$$
\begin{aligned}
H_{1}= & \operatorname{Re}\left(e^{\mathcal{A}_{1}+\overline{\mathcal{A}_{2}}} \frac{\partial \Phi}{\partial z} a \bar{c}-\widehat{\Psi}, e^{\mathcal{A}_{1}+\overline{\mathcal{A}_{2}}} \frac{\partial \Phi}{\partial z} a \bar{c}\right)_{L^{2}(\widetilde{\Gamma})} \\
& +\operatorname{Re}\left(e^{\mathcal{B}_{1}+\overline{\mathcal{B}}_{2}} \frac{\partial \bar{\Phi}}{\partial \bar{z}} \bar{b} d-\widehat{\widetilde{\Psi}}, e^{\mathcal{B}_{1}+\overline{\mathcal{B}}_{2}} \frac{\partial \bar{\Phi}}{\partial \bar{z}} \bar{b} d\right)_{L^{2}(\widetilde{\Gamma})}
\end{aligned}
$$




$$
\begin{aligned}
= & \operatorname{Re}\left(\left(\nu_{1}-i \nu_{2}\right) \bar{P}, e^{\mathcal{A}_{1}+\overline{\mathcal{A}_{2}}} \frac{\partial \Phi}{\partial z} a \bar{c}\right)_{L^{2}(\widetilde{\Gamma})} \\
& +\operatorname{Re}\left(\left(\nu_{1}+i \nu_{2}\right) \overline{\widetilde{P}}, e^{\mathcal{B}_{1}+\overline{\mathcal{B}_{2}}} \frac{\partial \bar{\Phi}}{\partial \bar{b}} d\right)_{L^{2}(\widetilde{\Gamma})} \\
= & 2 \operatorname{Re}\left(\left(\nu_{1}-i \nu_{2}\right) \overline{\left(\Phi_{0}+i \Psi_{0}\right)}, e^{\mathcal{A}_{1}+\overline{\mathcal{A}_{2}}} \frac{\partial \Phi}{\partial z} a \bar{c}\right)_{L^{2}(\widetilde{\Gamma})} \\
& +2 \operatorname{Re}\left(\left(\nu_{1}+i \nu_{2}\right)\left(\Phi_{0}-i \Psi_{0}\right), e^{\mathcal{B}_{1}+\overline{\mathcal{B}_{2}}} \frac{\partial \bar{\Phi}}{\partial \bar{z}} d\right)_{L^{2}(\widetilde{\Gamma})}
\end{aligned}
$$

We can rewrite

$$
\begin{aligned}
& 2 \operatorname{Re}\left(\left(\nu_{1}-i \nu_{2}\right) \overline{\Phi_{0}}, e^{\mathcal{A}_{1}+\overline{\mathcal{A}_{2}}} \frac{\partial \Phi}{\partial z} a \bar{c}\right)_{L^{2}(\widetilde{\Gamma})} \\
& +2 \operatorname{Re}\left(\left(\nu_{1}+i \nu_{2}\right) \Phi_{0}, e^{\mathcal{B}_{1}+\overline{\mathcal{B}_{2}}} \frac{\partial \bar{\Phi}}{\partial \bar{z}} \bar{b} d\right)_{L^{2}(\widetilde{\Gamma})} \\
& =\mathfrak{I}\left(\Phi, \Phi_{0} a, b, c, \Phi_{0} d\right)+\overline{\mathfrak{I}\left(\Phi, \Phi_{0} a, b, c, \Phi_{0} d\right)}
\end{aligned}
$$

and

$$
\begin{aligned}
& 2 \operatorname{Re}\left(\left(\nu_{1}-i \nu_{2}\right) \overline{\left(i \Psi_{0}\right)}, e^{\mathcal{A}_{1}+\overline{\mathcal{A}_{2}}} \frac{\partial \Phi}{\partial z} a \bar{c}\right)_{L^{2}(\widetilde{\Gamma})} \\
& +2 \operatorname{Re}\left(\left(\nu_{1}+i \nu_{2}\right)\left(-i \Psi_{0}\right), e^{\mathcal{B}_{1}+\overline{\mathcal{B}_{2}}} \frac{\partial \bar{\Phi}}{\partial \bar{z}} \bar{b} d\right)_{L^{2}(\widetilde{\Gamma})} \\
& =-2 \operatorname{Im}\left(\left(\nu_{1}-i \nu_{2}\right) \bar{a} c \overline{\Psi_{0}}, e^{\mathcal{A}_{1}+\overline{\mathcal{A}_{2}}} \frac{\partial \Phi}{\partial z}\right)_{L^{2}(\widetilde{\Gamma})} \\
& -2 \operatorname{Im}\left(\left(\nu_{1}+i \nu_{2}\right) b \bar{d} \Psi_{0}, e^{\mathcal{B}_{1}+\overline{\mathcal{B}_{2}}} \frac{\partial \bar{\Phi}}{\partial \bar{z}}\right)_{L^{2}(\widetilde{\Gamma})} \\
& =-\frac{1}{i} \int_{\widetilde{\Gamma}}\left(\left(\nu_{1}-i \nu_{2}\right) \bar{a} c \overline{\Psi_{0}} \frac{\partial \bar{\Phi}}{\partial \bar{z}} e^{\overline{\mathcal{A}_{1}}-\mathcal{A}_{2}}-\left(\nu_{1}+i \nu_{2}\right) a \bar{c} \Psi_{0} \frac{\partial \Phi}{\partial z} e^{\mathcal{A}_{1}+\overline{\mathcal{A}_{2}}}\right) d \sigma \\
& -\frac{1}{i} \int_{\widetilde{\Gamma}}\left(\left(\nu_{1}+i \nu_{2}\right) b \bar{d} \Psi_{0} \frac{\partial \Phi}{\partial z} e^{\overline{\mathcal{B}_{1}}+\mathcal{B}_{2}}-\left(\nu_{1}-i \nu_{2}\right) \bar{b} d \overline{\Psi_{0}} \frac{\partial \bar{\Phi}}{\partial \bar{z}} e^{\mathcal{B}_{1}+\overline{\mathcal{B}_{2}}}\right) d \sigma \\
& =\frac{1}{i}\left(\mathfrak{I}\left(\Phi, a \Psi_{0}, b, c, d \Psi_{0}\right)-\overline{\mathfrak{I}\left(\Phi, a \Psi_{0}, b, c, d \Psi_{0}\right)}\right) .
\end{aligned}
$$

Then by (5.42), (5.45), (5.46) and Proposition 5.3, we see that $H_{1}=0$. Taking into account $(5.44)$, we obtain $J(\widehat{\Psi}, \widehat{\widetilde{\Psi}})=0$. Consequently, setting $\Theta=\widehat{\Psi} /\left(\partial_{z} \Phi a \bar{c}\right)$ and $\widetilde{\Theta}=\widehat{\widetilde{\Psi}} /\left(\overline{\partial_{z} \Phi} d \bar{b}\right)$, we obtain (5.34).

Observe that

$$
\left(\nu_{1}+i \nu_{2}\right) \frac{\partial \Phi}{\partial z}=-\left(\nu_{1}-i \nu_{2}\right) \frac{\partial \bar{\Phi}}{\partial \bar{z}} \quad \text { on } \Gamma_{0}
$$


In order to see this, we argue as follows. We have

$$
\frac{\partial \Phi}{\partial \bar{z}}=\frac{1}{2}\left(\frac{\partial}{\partial x_{1}}+i \frac{\partial}{\partial x_{2}}\right)(\varphi+i \psi)=\frac{1}{2}\left(\frac{\partial \varphi}{\partial x_{1}}-\frac{\partial \psi}{\partial x_{2}}\right)+\frac{i}{2}\left(\frac{\partial \varphi}{\partial x_{2}}+\frac{\partial \psi}{\partial x_{1}}\right) .
$$

Hence $\frac{\partial \varphi}{\partial x_{1}}=\frac{\partial \psi}{\partial x_{2}}, \frac{\partial \varphi}{\partial x_{2}}=-\frac{\partial \psi}{\partial x_{1}}, \frac{\partial \varphi}{\partial \nu}=-\frac{\partial \psi}{\partial \vec{\tau}}$ and $\frac{\partial \psi}{\partial \nu}=\frac{\partial \varphi}{\partial \vec{\tau}}$. Observe that

$$
\begin{aligned}
& \left(\nu_{1}+i \nu_{2}\right) \frac{\partial}{\partial z}=\frac{1}{2}\left(\nu_{1} \frac{\partial}{\partial x_{1}}+\nu_{2} \frac{\partial}{\partial x_{2}}\right)+\frac{i}{2}\left(\nu_{2} \frac{\partial}{\partial x_{1}}-\nu_{1} \frac{\partial}{\partial x_{2}}\right)=\frac{1}{2}\left(\frac{\partial}{\partial \nu}+i \frac{\partial}{\partial \vec{\tau}}\right), \\
& \left(\nu_{1}-i \nu_{2}\right) \frac{\partial}{\partial \bar{z}}=\frac{1}{2}\left(\frac{\partial}{\partial \nu}-i \frac{\partial}{\partial \vec{\tau}}\right) .
\end{aligned}
$$

Hence

$$
\begin{aligned}
\left(\nu_{1}+i \nu_{2}\right) \frac{\partial \Phi}{\partial z} & =\frac{1}{2}\left(\frac{\partial}{\partial \nu}+i \frac{\partial}{\partial \vec{\tau}}\right)(\varphi+i \psi)=\frac{1}{2}\left(\frac{\partial \varphi}{\partial \nu}-\frac{\partial \psi}{\partial \vec{\tau}}\right)+\frac{i}{2}\left(\frac{\partial \varphi}{\partial \vec{\tau}}+\frac{\partial \psi}{\partial \nu}\right) \\
& =-\frac{\partial \psi}{\partial \vec{\tau}}+i \frac{\partial \varphi}{\partial \vec{\tau}} .
\end{aligned}
$$

Therefore

$$
\left(\nu_{1}-i \nu_{2}\right) \frac{\partial \bar{\Phi}}{\partial \bar{z}}=\frac{\overline{\partial \psi}+i \frac{\partial \varphi}{\partial \vec{\tau}}}{\partial \vec{\tau}}=-\frac{\partial \psi}{\partial \vec{\tau}}-i \frac{\partial \varphi}{\partial \vec{\tau}} .
$$

Taking into account that $\left.\psi\right|_{\Gamma_{0}}=0$, we obtain (5.47).

From (5.47), (4.3), (4.4), (4.31), (4.32) and (5.37), we obtain (5.35). The proof of the proposition is complete. In general $\Phi, a, b, c, d$ may have a finite number of zeros in $\Omega$. At those zeros $\Theta, \widetilde{\Theta}$ may have poles. On the other hand observe that $\Theta, \widetilde{\Theta}$ are independent of the particular choice of the functions $\Phi, a, b, c, d$. Making small perturbations of these functions, we can shift the position of the zeros. Therefore we may assume that there are no poles for $\Theta, \widetilde{\Theta}$.

Thanks to Proposition 5.4, we can rewrite (5.33) as

$$
\begin{aligned}
& o(1 / \tau)=\sum_{k=1}^{3} \tau^{2-k} \widetilde{F}_{k} \\
- & \frac{\pi}{\tau}\left\{\left(\mathcal{Q}_{+} a \bar{b}\right)(\widetilde{x}) \Lambda_{+}(\tau, \widetilde{x}) e^{\left(\mathcal{A}_{1}+\overline{\mathcal{B}_{2}}\right)(\widetilde{x})}+\left(\mathcal{Q}_{-} d \bar{c}\right)(\widetilde{x}) \Lambda_{-}(\tau, \widetilde{x}) e^{\left(\mathcal{B}_{1}+\overline{\mathcal{A}_{2}}\right)(\widetilde{x})}\right\} \\
- & \frac{1}{\tau} \int_{\Gamma_{0}}\left(\nu_{1}+i \nu_{2}\right)\left(e^{\mathcal{A}_{1}+\overline{\mathcal{A}_{2}}}-\Theta\right) \frac{\partial \Phi}{\partial z} a(z) \overline{\left(c_{2,+} \Lambda_{+}(\tau, \widetilde{x})+c_{2,-} \Lambda_{-}(\tau, \widetilde{x})\right)} d \sigma \\
- & \frac{1}{\tau} \int_{\Gamma_{0}}\left(\nu_{1}-i \nu_{2}\right)\left(e^{\mathcal{B}_{1}+\overline{\mathcal{B}_{2}}}-\widetilde{\Theta}\right) \frac{\partial \bar{\Phi}}{\partial \bar{z}} \overline{b(z)}\left(d_{2,+} \Lambda_{+}(\tau, \widetilde{x})+d_{2,-} \Lambda_{-}(\tau, \widetilde{x})\right) d \sigma \\
- & \frac{1}{\tau} \int_{\Gamma_{0}}\left(\nu_{1}+i \nu_{2}\right)\left(e^{\mathcal{A}_{1}+\overline{\mathcal{A}_{2}}}-\Theta\right) \frac{\partial \Phi}{\partial z} \overline{c(\bar{z})}\left(a_{2,+} \Lambda_{+}(\tau, \widetilde{x})+a_{2,-} \Lambda_{-}(\tau, \widetilde{x})\right) d \sigma \\
- & \frac{1}{\tau} \int_{\Gamma_{0}}\left(\nu_{1}-i \nu_{2}\right)\left(e^{\mathcal{B}_{1}+\overline{\mathcal{B}_{2}}}-\widetilde{\Theta}\right) \frac{\partial \bar{\Phi}}{\partial \bar{z}} d(\bar{z}) \overline{\left(b_{2,+} \Lambda_{+}(\tau, \widetilde{x})+b_{2,-} \Lambda_{-}(\tau, \widetilde{x})\right)} d \sigma
\end{aligned}
$$




$$
\begin{aligned}
& -2 \int_{\Gamma_{0}}\left(\nu_{1}+i \nu_{2}\right)\left(e^{\mathcal{A}_{1}+\overline{\mathcal{A}_{2}}}-\Theta\right) \overline{c_{\tau}(\bar{z}) \mathfrak{G}_{1}(x, \tau) d \sigma} \\
& +2 \int_{\Gamma_{0}}\left(\nu_{1}-i \nu_{2}\right)\left(e^{\mathcal{B}_{1}+\overline{\mathcal{B}_{2}}}-\widetilde{\Theta}\right) d_{\tau}(\bar{z}) \overline{\mathfrak{G}_{4}(x, \tau)} d \sigma \\
& +2 \int_{\Gamma_{0}}\left(\nu_{1}+i \nu_{2}\right)\left(e^{\mathcal{A}_{1}+\overline{\mathcal{A}_{2}}}-\Theta\right) a_{\tau}(z) \overline{\mathfrak{G}_{3}(x, \tau)} d \sigma \\
& -2 \int_{\Gamma_{0}}\left(\nu_{1}-i \nu_{2}\right)\left(e^{\mathcal{B}_{1}+\overline{\mathcal{B}_{2}}}-\widetilde{\Theta}\right) \overline{b_{\tau}(z) \mathfrak{G}_{2}(x, \tau) d \sigma} \\
& +\frac{\Lambda_{-}(\tau, \widetilde{x})}{\tau} \frac{\overline{\partial g_{4}(\widetilde{x})}}{\partial z} e^{-\overline{\mathcal{B}_{2}}(\widetilde{x})}\left(\int_{\Gamma_{0}} \frac{\left(\nu_{1}-i \nu_{2}\right) d\left(e^{\mathcal{B}_{1}+\overline{\mathcal{B}_{2}}}-\widetilde{\Theta}\right)}{\widetilde{\widetilde{z}}-\bar{z}} d \sigma-2 \pi(d \widetilde{\Theta})(\widetilde{x})\right) \\
& -\frac{\Lambda_{-}(\tau, \widetilde{x})}{\tau} \frac{\partial g_{1}(\widetilde{x})}{\partial z} e^{-\mathcal{A}_{1}(\widetilde{x})}\left(\int_{\Gamma_{0}} \frac{\left(\nu_{1}+i \nu_{2}\right) \bar{c}\left(e^{\mathcal{A}_{1}+\overline{\mathcal{A}_{2}}}-\Theta\right)}{\widetilde{z}-z} d \sigma-2 \pi(\bar{c} \Theta)(\widetilde{x})\right) \\
& +\frac{\Lambda_{+}(\tau, \widetilde{x})}{\tau} \frac{\overline{\partial g_{3}(\widetilde{x})}}{\partial \bar{z}} e^{-\overline{\mathcal{A}_{2}}(\widetilde{x})}\left(\int_{\Gamma_{0}} \frac{\left(\nu_{1}+i \nu_{2}\right) a\left(e^{\mathcal{A}_{1}+\overline{\mathcal{A}_{2}}}-\Theta\right)}{\widetilde{z}-z} d \sigma-2 \pi(a \Theta)(\widetilde{x})\right) \\
& -\frac{\Lambda_{+}(\tau, \widetilde{x})}{\tau} \frac{\partial g_{2}(\widetilde{x})}{\partial \bar{z}} e^{-\mathcal{B}_{1}(\widetilde{x})}\left(\int_{\Gamma_{0}} \frac{\left(\nu_{1}-i \nu_{2}\right) \bar{b}\left(e^{\mathcal{B}_{1}+\overline{\mathcal{B}_{2}}}-\widetilde{\Theta}\right)}{\widetilde{\widetilde{z}}-\bar{z}} d \sigma-2 \pi(\bar{b} \Theta)(\widetilde{x})\right)
\end{aligned}
$$

Here $\widetilde{F}_{k}$ are some constants independent of $\tau$.

Then, using (4.15), (5.35) and (5.47), on $\Gamma_{0}$ we have

$$
\begin{gathered}
(5.49)-\left(\nu_{1}+i \nu_{2}\right) \frac{\partial \Phi}{\partial z}\left(e^{\mathcal{A}_{1}+\overline{\mathcal{A}_{2}}}-\Theta\right) \bar{c}\left(a_{2,+} e^{2 \tau i \psi(\widetilde{x})}+a_{2,-} e^{-2 \tau i \psi(\widetilde{x})}\right) \\
-\left(\nu_{1}-i \nu_{2}\right) \frac{\partial \Phi}{\partial \bar{z}}\left(e^{\mathcal{B}_{1}+\overline{\mathcal{B}_{2}}}-\widetilde{\Theta}\right) \bar{b}\left(d_{2,+} e^{2 \tau i \psi(\widetilde{x})}+d_{2,-} e^{-2 \tau i \psi(\widetilde{x})}\right) \\
=-\left(\nu_{1}+i \nu_{2}\right) \frac{\partial \Phi}{\partial z} \bar{c}\left(\left(e^{\mathcal{A}_{1}+\overline{\mathcal{A}_{2}}}-\Theta\right) a_{2,+} e^{2 \tau i \psi(\widetilde{x})}+\left(e^{\mathcal{B}_{1}+\overline{\mathcal{A}_{2}}}-\widetilde{\Theta} e^{\overline{\mathcal{A}_{2}}-\overline{\mathcal{B}_{2}}}\right) d_{2,+} e^{2 \tau i \psi(\widetilde{x})}\right)
\end{gathered}
$$$$
-\left(\nu_{1}-i \nu_{2}\right) \frac{\partial \bar{\Phi}}{\partial \bar{z}} \bar{b}\left(\left(e^{\mathcal{A}_{1}+\overline{\mathcal{B}_{2}}}-\Theta e^{\overline{\mathcal{B}_{2}}-\overline{\mathcal{A}_{2}}}\right) a_{2,-} e^{-2 \tau i \psi(\widetilde{x})}+\left(e^{\mathcal{B}_{1}+\overline{\mathcal{B}_{2}}}-\widetilde{\Theta}\right) d_{2,-} e^{-2 \tau i \psi(\widetilde{x})}\right)
$$$$
=-\left(\nu_{1}+i \nu_{2}\right) \frac{\partial \Phi}{\partial z} \bar{c}\left(\left(e^{\mathcal{A}_{1}+\overline{\mathcal{A}_{2}}}-\Theta\right) a_{2,+} e^{2 \tau i \psi(\widetilde{x})}+\left(e^{\mathcal{B}_{1}+\overline{\mathcal{A}_{2}}}-\Theta e^{-\mathcal{A}_{1}+\mathcal{B}_{1}}\right) d_{2,+} e^{2 \tau i \psi(\widetilde{x})}\right)
$$$$
-\left(\nu_{1}-i \nu_{2}\right) \frac{\partial \bar{\Phi}}{\partial \bar{z}} \bar{b}\left(\left(e^{\mathcal{A}_{1}+\overline{\mathcal{B}_{2}}}-\widetilde{\Theta} e^{\mathcal{A}_{1}-\mathcal{B}_{1}}\right) a_{2,-} e^{-2 \tau i \psi(\widetilde{x})}+\left(e^{\mathcal{B}_{1}+\overline{\mathcal{B}_{2}}}-\widetilde{\Theta}\right) d_{2,-} e^{-2 \tau i \psi(\widetilde{x})}\right)
$$

$=-\left(\nu_{1}+i \nu_{2}\right) \frac{\partial \Phi}{\partial z}\left(e^{\overline{\mathcal{A}_{2}}}-\Theta e^{-\mathcal{A}_{1}}\right) \bar{c} p_{+} e^{2 \tau i \psi(\widetilde{x})}-\left(\nu_{1}-i \nu_{2}\right) \frac{\partial \bar{\Phi}}{\partial \bar{z}}\left(e^{\overline{\mathcal{B}_{2}}}-\widetilde{\Theta} e^{-\mathcal{B}_{1}}\right) \bar{b} p_{-} e^{-2 \tau i \psi(\widetilde{x})}$

and

$$
\begin{aligned}
-\left(\nu_{1}+i \nu_{2}\right) & \left(e^{\mathcal{A}_{1}+\overline{\mathcal{A}_{2}}}-\Theta\right) \frac{\partial \Phi}{\partial z} a\left(\overline{c_{2,+} e^{2 \tau i \psi(\widetilde{x})}+c_{2,-} e^{-2 \tau i \psi(\widetilde{x})}}\right) \\
& -\left(\nu_{1}-i \nu_{2}\right)\left(e^{\mathcal{B}_{1}+\overline{\mathcal{B}_{2}}}-\widetilde{\Theta}\right) \frac{\partial \bar{\Phi}}{\partial \bar{z}} d\left(\overline{b_{2,+} e^{2 \tau i \psi(\widetilde{x})}+b_{2,-} e^{-2 \tau i \psi(\widetilde{x})}}\right)
\end{aligned}
$$




$$
\begin{aligned}
& =-\left(\nu_{1}+i \nu_{2}\right) \frac{\partial \Phi}{\partial z} a\left(\left(e^{\mathcal{A}_{1}+\overline{\mathcal{A}_{2}}}-\Theta\right) \overline{c_{2,-} e^{-2 \tau i \psi(\widetilde{x})}}+\left(e^{\mathcal{A}_{1}+\overline{\mathcal{B}_{2}}}-e^{-\mathcal{B}_{1}+\mathcal{A}_{1}} \widetilde{\Theta}\right) \overline{b_{2,-} e^{-2 \tau i \psi(\widetilde{x})}}\right) \\
& -\left(\nu_{1}-i \nu_{2}\right) \frac{\partial \bar{\Phi}}{\partial \bar{z}} d\left(\left(e^{\mathcal{B}_{1}+\overline{\mathcal{A}_{2}}}-\Theta e^{-\mathcal{A}_{1}+\mathcal{B}_{1}}\right) \overline{c_{2,+} e^{-2 \tau i \psi(\widetilde{x})}}+\left(e^{\mathcal{B}_{1}+\overline{\mathcal{B}_{2}}}-\widetilde{\Theta}\right) \overline{b_{2,+} e^{2 \tau i \psi(\widetilde{x})}}\right) \\
& =-\left(\nu_{1}+i \nu_{2}\right) \frac{\partial \Phi}{\partial z} a\left(\left(e^{\mathcal{A}_{1}+\overline{\mathcal{A}_{2}}}-\Theta\right) \overline{c_{2,-} e^{-2 \tau i \psi(\widetilde{x})}}+\left(e^{\mathcal{A}_{1}+\overline{\mathcal{B}_{2}}}-e^{\overline{\mathcal{B}_{2}}}-\overline{\mathcal{A}_{2}} \Theta\right) \overline{b b_{2,-} e^{-2 \tau i \psi(\widetilde{x})}}\right) \\
& -\left(\nu_{1}-i \nu_{2}\right) \frac{\partial \bar{\Phi}}{\partial \bar{z}} d\left(\left(e^{\mathcal{B}_{1}+\overline{\mathcal{A}_{2}}}-\Theta e^{\overline{\mathcal{A}_{2}}-\overline{\mathcal{B}_{2}}}\right) \overline{c_{2,+} e^{2 \tau i \psi(\widetilde{x})}}+\left(e^{\mathcal{B}_{1}+\overline{\mathcal{B}_{2}}}-\widetilde{\Theta}\right) \overline{b_{2,+} e^{2 \tau i \psi(\widetilde{x})}}\right) \\
& =-\left(\nu_{1}+i \nu_{2}\right) \frac{\partial \Phi}{\partial z}\left(e^{\mathcal{A}_{1}}-\Theta e^{-\overline{\mathcal{A}_{2}}}\right) a \overline{\widetilde{p}_{-} e^{-2 \tau i \psi(\widetilde{x})}}-\left(\nu_{1}-i \nu_{2}\right) \frac{\partial \bar{\Phi}}{\partial \bar{z}}\left(e^{\mathcal{B}_{1}}-\widetilde{\Theta} e^{-\overline{\mathcal{B}_{2}}}\right) d \overline{\widetilde{p}_{+} e^{2 \tau i \psi(\widetilde{x})}} .
\end{aligned}
$$

Using (5.49), (5.50) and Proposition 8.2, we rewrite (5.48) as

$$
\begin{aligned}
& o(1 / \tau)=\sum_{k=1}^{3} \tau^{2-k} \widetilde{F}_{k} \\
& -\frac{\pi}{\tau}\left\{\left(\mathcal{Q}_{+} a \bar{b}\right)(\widetilde{x}) \Lambda_{+}(\tau, \widetilde{x}) e^{\left(\mathcal{A}_{1}+\overline{\mathcal{B}_{2}}\right)(\widetilde{x})}+\left(\mathcal{Q}_{-} d \bar{c}\right)(\widetilde{x}) \Lambda_{-}(\tau, \widetilde{x}) e^{\left(\mathcal{B}_{1}+\overline{\mathcal{A}_{2}}\right)(\widetilde{x})}\right\} \\
& -\frac{\Lambda_{+}(\tau, \widetilde{x})}{\tau} \int_{\Gamma_{0}}\left(\nu_{1}+i \nu_{2}\right)\left(e^{\mathcal{A}_{1}+\overline{\mathcal{A}_{2}}}-\Theta\right) a \frac{\overline{\left(\partial_{z} g_{3} e^{-\mathcal{A}_{2}}\right)(\widetilde{x})}}{\widetilde{z}-z} d \sigma \\
& +\frac{\Lambda_{-}(\tau, \widetilde{x})}{\tau} \int_{\Gamma_{0}}\left(\nu_{1}+i \nu_{2}\right)\left(e^{\mathcal{A}_{1}+\overline{\mathcal{A}_{2}}}-\Theta\right) \overline{c(\bar{z})} \frac{\left(\partial_{z} g_{1} e^{-\mathcal{A}_{1}}\right)(\widetilde{x})}{\widetilde{z}-z} d \sigma \\
& -\frac{\Lambda_{-}(\tau, \widetilde{x})}{\tau} \int_{\Gamma_{0}}\left(\nu_{1}-i \nu_{2}\right)\left(e^{\mathcal{B}_{1}+\overline{\mathcal{B}_{2}}}-\widetilde{\Theta}\right) d \frac{\overline{\left(\partial_{\bar{z}} g_{4} e^{-\mathcal{B}_{2}}\right)(\widetilde{x})}}{\overline{\widetilde{z}}-\bar{z}} d \sigma \\
& +\frac{\Lambda_{+}(\tau, \widetilde{x})}{\tau} \int_{\Gamma_{0}}\left(\nu_{1}-i \nu_{2}\right)\left(e^{\mathcal{B}_{1}+\overline{\mathcal{B}_{2}}}-\widetilde{\Theta}\right) \overline{b(z)} \frac{\left(\partial_{\bar{z}} g_{2} e^{-\mathcal{B}_{1}}\right)(\widetilde{x})}{\overline{\widetilde{z}}-\bar{z}} d \sigma \\
& +\frac{\Lambda_{-}(\tau, \widetilde{x})}{\tau} \frac{\overline{\partial g_{4}(\widetilde{x})}}{\partial z} e^{-\overline{\mathcal{B}_{2}}(\widetilde{x})}\left(\int_{\Gamma_{0}} \frac{\left(\nu_{1}-i \nu_{2}\right) d\left(e^{\mathcal{B}_{1}+\overline{\mathcal{B}_{2}}}-\widetilde{\Theta}\right)}{\overline{\widetilde{z}}-\bar{z}} d \sigma-2 \pi(d \widetilde{\Theta})(\widetilde{x})\right) \\
& -\frac{\Lambda_{-}(\tau, \widetilde{x})}{\tau} \frac{\partial g_{1}(\widetilde{x})}{\partial z} e^{-\mathcal{A}_{1}(\widetilde{x})}\left(\int_{\Gamma_{0}} \frac{\left(\nu_{1}+i \nu_{2}\right) \bar{c}\left(e^{\mathcal{A}_{1}+\overline{\mathcal{A}_{2}}}-\Theta\right)}{\widetilde{z}-z} d \sigma-2 \pi(\bar{c} \Theta)(\widetilde{x})\right) \\
& +\frac{\Lambda_{+}(\tau, \widetilde{x})}{\tau} \frac{\overline{\partial g_{3}(\widetilde{x})}}{\partial \bar{z}} e^{-\overline{\mathcal{A}_{2}}(\widetilde{x})}\left(\int_{\Gamma_{0}} \frac{\left(\nu_{1}+i \nu_{2}\right) a\left(e^{\mathcal{A}_{1}+\overline{\mathcal{A}_{2}}}-\Theta\right)}{\widetilde{z}-z} d \sigma-2 \pi(a \Theta)(\widetilde{x})\right) \\
& -\frac{\Lambda_{+}(\tau, \widetilde{x})}{\tau} \frac{\partial g_{2}(\widetilde{x})}{\partial \bar{z}} e^{-\mathcal{B}_{1}(\widetilde{x})}\left(\int_{\Gamma_{0}} \frac{\left(\nu_{1}-i \nu_{2}\right) \bar{b}\left(e^{\mathcal{B}_{1}+\overline{\mathcal{B}_{2}}}-\widetilde{\Theta}\right)}{\overline{\widetilde{z}}-\bar{z}} d \sigma-2 \pi(\bar{b} \widetilde{\Theta})(\widetilde{x})\right) .
\end{aligned}
$$

Let $\eta$ be a smooth function such that $\eta$ is zero in some neighborhood of $\partial \Omega$ and $\eta(\widetilde{x})=-1$. Observe that the partial Cauchy data of the operators $L_{2}(x, D)$ and the operator $e^{-s \eta} L_{1}(x, D) e^{s \eta}$ are exactly the same. Therefore we have the analog of (5.51) for these two operators with $\mathcal{A}_{1}$ and $\mathcal{B}_{1}$ replaced by $\mathcal{A}_{1}-s \eta$ and $\mathcal{B}_{1}-s \eta$. The coefficients $A_{1}, B_{1}$ should be replaced by $A_{1}+2 s \frac{\partial \eta}{\partial \bar{z}}, B_{1}+2 s \frac{\partial \eta}{\partial z}$. The functions $\mathcal{Q}_{ \pm}$do not change. The function $q_{1}$ should be replaced by $q_{1}+s \Delta \eta+s^{2}|\nabla \eta|^{2}+$ 
$2 s A_{1} \frac{\partial \eta}{\partial z}+2 s B_{1} \frac{\partial \eta}{\partial \bar{z}}$. The functions $g_{1}, g_{2}$ should be replaced by $e^{-s \eta} g_{1}, e^{-s \eta} g_{2}$. The functions $\Theta, \widetilde{\Theta}$ remain unchanged since $\eta$ is zero in a neighborhood of the boundary and therefore $\mathcal{A}_{j}, \mathcal{B}_{j}$ remain unchanged in a neighborhood of the boundary.

Since $\psi(\widetilde{x}) \neq 0$, all $\widetilde{F}_{k}$ are zero. The third till the tenth terms on the righthand side of (5.51) are independent of $s$. On the other hand, the term in the second line of (5.51) has the form

$$
-\frac{e^{s} \pi}{\tau}\left\{\left(\mathcal{Q}_{+} a \bar{b}\right)(\widetilde{x}) \Lambda_{+}(\tau, \widetilde{x}) e^{\left(\mathcal{A}_{1}+\overline{\mathcal{B}_{2}}\right)(\widetilde{x})}+\left(\mathcal{Q}_{-} d \bar{c}\right)(\widetilde{x}) \Lambda_{-}(\tau, \widetilde{x}) e^{\left(\mathcal{B}_{1}+\overline{\mathcal{A}_{2}}\right)(\widetilde{x})}\right\}
$$

This immediately implies that $\left(\mathcal{Q}_{+} a \bar{b}\right)(\widetilde{x})=\left(\mathcal{Q}_{-} d \bar{c}\right)(\widetilde{x})=0$.

\section{$\S 6$. Proof of Theorem 1.1}

Suppose that we have two operators

$$
L_{1}(x, D)=\Delta_{\mathbf{g}_{1}}+2 A_{1} \frac{\partial}{\partial z}+2 B_{1} \frac{\partial}{\partial \bar{z}}+q_{1}
$$

and

$$
L_{2}(x, D)=\Delta_{\mathbf{g}_{2}}+2 A_{2} \frac{\partial}{\partial z}+2 B_{2} \frac{\partial}{\partial \bar{z}}+q_{2}
$$

with the same partial Cauchy data. Multiplying the metric $\mathbf{g}_{2}$, if necessary, by some positive smooth function $\widetilde{\beta}$, we may assume that

$$
\left.\frac{\partial^{\ell}}{\partial \nu^{\ell}}\left(g_{1}^{j k}-g_{2}^{j k}\right)\right|_{\widetilde{\Gamma}}=0 \quad \forall \ell \in\{0,1\} \text { and } \forall j, k \in\{1,2\} .
$$

We note that $\left\{g_{1}^{j k}\right\}$ denotes the inverse matrix to $\mathbf{g}_{1}=\left\{g_{1, j k}\right\}$. Without loss of generality, we may assume that there exists a smooth positive function $\mu_{2}$ such that $\mathbf{g}_{2}=\mu_{2} I$. Indeed, using isothermal coordinates we make a change of variables in the operator $L_{2}(x, D)$ such that $\mathbf{g}_{2}=\mu_{2} I$. Then we make the same changes of variables in the operator $L_{1}(x, D)$. The partial Cauchy data of both operators obtained by this change of variables are the same.

Let $\omega$ be a subdomain in $\mathbb{R}^{2}$ such that $\Omega \cap \omega=\emptyset, \partial \omega \cap \partial \Omega=\widetilde{\Gamma}$ and the boundary of the domain $\widetilde{\Omega}=\operatorname{Int}(\overline{\Omega \cup \omega})$ is smooth. We extend $\mu_{2}$ onto $\widetilde{\Omega}$ as a smooth positive function and set $g_{1}^{j k}=\left(1 / \mu_{2}\right) I$ in $\omega$. By $(6.1)$, we have $\mathbf{g}_{1} \in C^{1}(\bar{\Omega})$. There exists an isothermal mapping $\chi_{1}=\left(\chi_{1,1}, \chi_{1,2}\right)$ such that the operator $L_{1}(x, D)$ is transformed to

$$
Q_{1}(y, D)=\frac{1}{\mu_{1}} \Delta+2 C_{1} \frac{\partial}{\partial z}+2 D_{1} \frac{\partial}{\partial \bar{z}}+r_{1}, \quad y \in \chi_{1}(\widetilde{\Omega}),
$$


where $\mu_{1}$ is a smooth positive function in $\chi_{1}(\widetilde{\Omega})$ and $C_{1}, D_{1}, r_{1}$ are some smooth complex-valued functions. Consider a solution to the boundary value problem

$$
Q_{1}(y, D) w=0 \quad \text { in } \chi_{1}(\widetilde{\Omega}),\left.\quad w\right|_{\chi_{1}\left(\Gamma_{0}\right)}=0,
$$

of the form (4.2) with a holomorphic weight function $\Phi_{1}$. Then the function $u_{1}(x)=$ $w\left(\chi_{1}(x)\right)$ is a solution to

$$
L_{1}(x, D) u_{1}=0 \quad \text { in } \widetilde{\Omega},\left.\quad u_{1}\right|_{\Gamma_{0}}=0 .
$$

Since the partial Cauchy data for the operators $L_{1}(x, D)$ and $L_{2}(x, D)$ are the same, there exists a function $u_{2}$ such that

$$
L_{2}(x, D) u_{2}=0 \quad \text { in } \Omega,\left.\quad u_{2}\right|_{\Gamma_{0}}=0,\left.\quad\left(\frac{\partial u_{1}}{\partial \nu_{\mathbf{g}_{1}}}-\frac{\partial u_{2}}{\partial \nu_{\mathbf{g}_{2}}}\right)\right|_{\widetilde{\Gamma}}=0 .
$$

Using (6.1) and (6.3) we extend $u_{2}$ to a function $u_{2} \in H^{1}(\widetilde{\Omega})$ such that

$$
\left.u_{1}\right|_{\omega}=\left.u_{2}\right|_{\omega} .
$$

Let $\varphi_{2}$ be a harmonic function in $\widetilde{\Omega}$ such that

$$
\left.\frac{\partial \varphi_{2}}{\partial \nu}\right|_{\Gamma_{0}}=0, \quad \varphi_{2}=\operatorname{Re} \Phi_{1} \circ \chi_{1} \quad \text { on } \partial \widetilde{\Omega} \backslash \Gamma_{0} .
$$

We claim that

$$
\varphi_{2}=\operatorname{Re} \Phi_{1} \circ \chi_{1} \quad \text { on } \omega .
$$

A difficulty comes from the fact that the function $\varphi_{2}$ is continuous on $\bar{\Omega}$ (see e.g. [25]) but the derivatives of $\varphi_{2}$ may be discontinuous at some points of $\partial \Gamma_{0}$. First we observe that it suffices to prove (6.5) for the functions such that $\operatorname{Im} \Phi_{1}=0$ in some open set $\mathcal{O}_{\Phi_{1}} \subset \partial \chi_{1}(\widetilde{\Omega})$ such that $\overline{\chi_{1}\left(\Gamma_{0}\right)} \subset \mathcal{O}_{\Phi_{1}}$. Indeed, without loss of generality, assume that $\partial \widetilde{\Omega} \backslash \Gamma_{0}$ is an arc with two endpoints $x_{ \pm}$. We choose sequences $\left\{x_{\epsilon,-}\right\},\left\{x_{\epsilon,+}\right\} \subset \partial \widetilde{\Omega} \backslash \Gamma_{0}$ such that $x_{\epsilon, \pm} \rightarrow x_{ \pm}$as $\epsilon \rightarrow 0$. Consider a sequence $\left\{\Phi_{1, \epsilon}\right\}_{\epsilon \in(0,1)}$ of holomorphic functions such that

$$
\begin{aligned}
& \frac{\partial \Phi_{1, \epsilon}}{\partial \bar{z}}=0 \quad \text { in } \chi_{1}(\widetilde{\Omega}),\left.\quad \operatorname{Im} \Phi_{1, \epsilon}\right|_{\chi_{1}\left(\Gamma_{0, \epsilon}\right)}=0, \\
& \partial_{z}^{2} \Phi_{1, \epsilon}(z) \neq 0 \quad \forall z \in\left\{z \mid \partial_{z} \Phi_{1, \epsilon}(z)=0\right\}, \\
& \Phi_{1, \epsilon} \rightarrow \Phi_{1} \quad \text { in } C^{1}\left(\overline{\widetilde{\Gamma}}_{\epsilon}\right),
\end{aligned}
$$

where $\overline{\widetilde{\Gamma}}_{\epsilon} \subset \widetilde{\Gamma}$ is the arc between $x_{\epsilon,-}, x_{\epsilon,+}$ and $\Gamma_{0, \epsilon}=\partial \widetilde{\Omega} \backslash \widetilde{\Gamma}_{\epsilon}$. We define $\varphi_{2, \epsilon}$ by

$$
\left.\frac{\partial \varphi_{2, \epsilon}}{\partial \nu}\right|_{\Gamma_{0, \epsilon}}=0, \quad \varphi_{2, \epsilon}=\operatorname{Re} \Phi_{1, \epsilon} \circ \chi_{1} \quad \text { on } \partial \widetilde{\Omega} \backslash \Gamma_{0, \epsilon} .
$$


First we assume that

$$
\varphi_{2, \epsilon}=\operatorname{Re} \Phi_{1, \epsilon} \circ \chi_{1} \quad \text { on } \omega
$$

Passing to the limit in the above equality, we obtain (6.5).

Now we prove that (6.6) indeed holds. Let $\Phi_{1, \epsilon}$ be one of the functions in the sequence $\left\{\Phi_{1, \epsilon}\right\}_{\epsilon \in(0,1)}$. Consider a sequence of domains $\widetilde{\Omega}_{\epsilon}$ such that $\widetilde{\Omega}_{\epsilon} \subset \widetilde{\Omega}$, $\partial \widetilde{\Omega}_{\epsilon} \cap \partial \widetilde{\Omega}=\Gamma_{0}$ and $\operatorname{dist}\left(\partial \widetilde{\Omega}_{\epsilon} \backslash \Gamma_{0}, \widetilde{\Gamma}\right) \rightarrow 0$ as $\epsilon \rightarrow 0$. Then $\varphi_{2, \epsilon}$ is smooth in $\widetilde{\Omega}_{\epsilon}$. Let us take as $u_{1}$ the complex geometrical optics solution constructed in the previous sections. Thanks to the Carleman estimate (2.25) there exists $\tau_{0}=\tau_{0}(\epsilon)$ such that

$$
\left\|e^{-\tau \varphi_{2, \epsilon}} u_{2}\right\|_{L^{2}\left(\widetilde{\Omega}_{\epsilon}\right)} \leq C_{0}\left|\tau e^{\delta_{\epsilon}|\tau|}\right| \quad \forall|\tau| \geq \tau_{0},
$$

where $C_{0}=C_{0}(\epsilon)$ is independent of $\tau$ and $\delta_{\epsilon} \rightarrow 0$ as $\epsilon \rightarrow 0$. On the other hand, $u_{1}=e^{\tau \operatorname{Re} \Phi_{1, \epsilon} \circ \chi_{1}}\left(\left(a_{\tau} e^{\mathcal{C}_{1}+i \tau \operatorname{Im} \Phi_{1, \epsilon}}+b_{\tau} e^{\mathcal{D}_{1}-i \tau \operatorname{Im} \Phi_{1, \epsilon}}\right) \circ \chi_{1}+O(1 / \tau)\right)$. Here we note that $\mathcal{C}_{1}, \mathcal{D}_{1} \in C^{6+\alpha}\left(\widetilde{\Omega}_{\epsilon}\right)$ are defined similarly to $(3.2)$ :

$$
2 \frac{\partial \mathcal{C}_{1}}{\partial \bar{z}}=-C_{1} \quad \text { in } \widetilde{\Omega}_{\epsilon},\left.\quad \operatorname{Im} \mathcal{C}_{1}\right|_{\Gamma_{0}}=0, \quad 2 \frac{\partial \mathcal{D}_{1}}{\partial z}=-D_{1} \quad \text { in } \widetilde{\Omega}_{\epsilon},\left.\quad \operatorname{Im} \mathcal{D}_{1}\right|_{\Gamma_{0}}=0 .
$$

Then by (6.4),

$$
\begin{array}{r}
u_{2}=e^{\tau \operatorname{Re} \Phi_{1, \epsilon} \circ \chi_{1}}\left(\left(a_{\tau} e^{\mathcal{C}_{1}+i \tau \operatorname{Im} \Phi_{1, \epsilon}}+b_{\tau} e^{\mathcal{D}_{1}-i \tau \operatorname{Im} \Phi_{1, \epsilon}}\right) \circ \chi_{1}+O(1 / \tau)\right) \\
\forall x \in \omega .
\end{array}
$$

This equality implies (6.6) immediately. Indeed, suppose that for some $\widehat{x} \in \omega$,

$$
\varphi_{2, \epsilon}(\widehat{x}) \neq \operatorname{Re} \Phi_{1, \epsilon} \circ \chi_{1}(\widehat{x}) .
$$

Then there exists a ball $B\left(\widehat{x}, \delta^{\prime}\right) \subset \omega$ such that

$$
\left|\varphi_{2, \epsilon}(x)-\operatorname{Re} \Phi_{1, \epsilon} \circ \chi_{1}(x)\right|>\alpha^{\prime}>0 \quad \forall x \in \overline{B\left(\widehat{x}, \delta^{\prime}\right)} .
$$

Fix a positive $\epsilon_{1}$ such that $\overline{B\left(\widehat{x}, \delta^{\prime}\right)} \subset \Omega_{\epsilon_{1}}$ and $2 \delta_{\epsilon_{1}}<\alpha^{\prime}$. From (6.8), by (6.7) and (6.10), for some positive $C^{\prime}$ we have

$$
\begin{aligned}
& C^{\prime} e^{|\tau| \alpha^{\prime}} \operatorname{Vol}\left(B\left(\widehat{x}, \delta^{\prime}\right)\right)^{1 / 2} \\
& \quad \leq\left\|e^{\tau\left(\operatorname{Re} \Phi_{1, \epsilon} \circ \chi_{1}-\varphi_{2, \epsilon}\right)}\left(\left(a_{\tau} e^{\mathcal{C}_{1}+i \tau \operatorname{Im} \Phi_{1, \epsilon}}+b_{\tau} e^{\mathcal{D}-i \tau \operatorname{Im} \Phi_{1, \epsilon}}\right) \circ \chi_{1}+O(1 / \tau)\right)\right\|_{L^{2}\left(B\left(\widehat{x}, \delta^{\prime}\right)\right)} \\
& \quad=\left\|e^{-\tau \varphi_{2, \epsilon}} u_{2}\right\|_{L^{2}\left(B\left(\widehat{x}, \delta^{\prime}\right)\right)} \leq C_{0}|\tau| e^{\delta_{\epsilon}|\tau|}
\end{aligned}
$$

where $\tau>\tau_{0}$ if $\varphi_{2, \epsilon}(\widehat{x})<\operatorname{Re} \Phi_{1, \epsilon} \circ \chi_{1}(\widehat{x})$ and $\tau<-\tau_{0}$ if $\varphi_{2, \epsilon}(\widehat{x})>\operatorname{Re} \Phi_{1, \epsilon} \circ \chi_{1}(\widehat{x})$. The above inequality contradicts (6.10) and therefore (6.9). 
Let $\Xi=\chi_{1,1}+i \chi_{1,2}$. Using the Cauchy-Riemann equations, we construct a multivalued function $\psi_{2}$ such that $\Phi_{2}=\varphi_{2}+i \psi_{2}$ is holomorphic on the Riemann surface associated with $\widetilde{\Omega}$. Moreover we take $\Phi_{1}$ which can be holomorphically extended onto some domain $\mathcal{O}$ such that $\chi_{1}(\widetilde{\Omega}) \subset \mathcal{O}$. Observe that

$$
\Phi_{2}=\Phi_{1} \circ \Xi \text { on } \omega \text {. }
$$

Then $\Xi=\Phi_{1}^{-1} \circ \Phi_{2}$ on $\omega$.

We claim that $\Xi$ can be extended to a single-valued holomorphic function $\widetilde{\Xi}$ on $\widetilde{\Omega}$ such that $\widetilde{\Xi}: \widetilde{\Omega} \rightarrow \chi_{1}(\widetilde{\Omega}), \widetilde{\Xi}(\widetilde{\Omega})=\chi_{1}(\widetilde{\Omega})$ and $\partial_{z} \widetilde{\Xi} \neq 0$. First we show that $\Xi$ can be extended along any curve connecting two points in $\Omega$. Indeed, let $\gamma$ be a continuous curve connecting a point $z_{1}$ in $\omega$ and a point $z_{2}$ in $\Omega$ such that $\Xi$ cannot be extended along $\gamma$. Consider a parametrization of $\gamma$ such that we are moving from $z_{1}=\gamma(0)$ to $z_{2}=\gamma(1)$. Let $\widehat{z}=\gamma(\kappa)$ be the first point on $\gamma$ around which the holomorphic continuation of $\Xi$ is impossible. Consider the function $\Phi_{1}$ such that $\left\{z \mid \partial_{z} \Phi_{1}=0\right\} \cap\{z \mid z=\widetilde{\Xi}(\gamma(s)), s \in[0, \kappa]\}=\emptyset$. Observe that

$$
\Phi_{2}(\gamma(s))=\Phi_{1} \circ \Xi(\gamma(s)) \quad \forall s \in[0, \kappa] .
$$

Indeed, let $\widehat{s}=\sup _{s \in X} s$ where $X=\left\{s \mid\right.$ there exists $\delta(s)>0$ such that $\Phi_{2}(z)=$ $\Phi_{1} \circ \Xi(z)$ for all $\left.z \in B(\gamma(s), \delta)\right\}$. Let $\widehat{s}<\kappa$. Since $\partial_{z} \Phi_{1}(\gamma(\widehat{s})) \neq 0$, we see that $\Phi_{1}^{-1} \circ \Phi_{2}$ is holomorphic with a domain which contains a ball centered at $\gamma(\widehat{s})$. Since $\widetilde{\Xi}=\Phi_{1}^{-1} \circ \Phi_{2}$ in some open set, this equality holds true on this ball, which contradicts the definition of $\widehat{s}$.

Now we consider the situation at the point $\widehat{z}$. Since we cannot extend $\Xi$ around this point, we have $\Pi=\left\{\widetilde{z} \mid \Phi_{1}(\widetilde{z})=\Phi_{2}(\widehat{z})\right\} \subset \chi_{1}(\partial \widetilde{\Omega})$. Since $\partial_{z} \Phi_{1}(\widetilde{z}) \neq 0$, we can extend $\Xi$ onto some ball centered at $\widehat{z}$. (Of course such an extension might not be one which we are looking for, since $\widetilde{\Xi}: \widetilde{\Omega} \rightarrow \chi_{1}(\widetilde{\Omega})$ might not be valid.) Consider a perturbation $\Phi_{1}+\epsilon \Psi_{1}$ of $\Phi_{1}$, where $\Psi_{1}$ is a smooth holomorphic function in $\mathcal{O}$ such that $\left.\operatorname{Im} \Psi_{1}\right|_{\chi_{1}\left(\Gamma_{0}\right) \cup \Pi}=0$. This perturbation generates a perturbation $\Phi_{2}+\epsilon \Psi_{2}$ of $\Phi_{2}$, where

$$
\Delta \operatorname{Re} \Psi_{2}=0 \quad \text { in } \widetilde{\Omega},\left.\quad \frac{\partial \operatorname{Re} \Psi_{2}}{\partial \nu}\right|_{\Gamma_{0}}=0, \quad \operatorname{Re} \Psi_{2}=\operatorname{Re} \Psi_{1} \circ \chi_{1} \quad \text { on } \partial \widetilde{\Omega} \backslash \Gamma_{0} .
$$

For these new functions we still have

$$
\Phi_{2}+\epsilon \Psi_{2}=\left(\Phi_{1}+\epsilon \Psi_{1}\right) \circ \Xi \text { on } \omega .
$$

For all sufficiently small $\epsilon$, the function $\Phi_{1}+\epsilon \Psi_{1}$ does not have a critical point in $\widetilde{\Omega}$. Therefore the function $\left(\Phi_{1}+\epsilon \Psi_{1}\right)^{-1} \circ\left(\Phi_{2}+\epsilon \Psi_{2}\right)$ can be holomorphically continued along $\gamma$ up to $\widehat{z}$. Denote this extension onto some ball centered at $\widehat{z}$ 
by $\widetilde{\Xi}_{\epsilon}$. Obviously $\widetilde{\Xi}_{\epsilon}=\widetilde{\Xi}$. Choosing $\Psi_{1}$ such that $\operatorname{Im} \Psi_{2}(\widehat{z}) \neq \operatorname{Im} \Psi_{1}(\widetilde{\Xi}(\widehat{z}))$, we conclude that this equality is impossible.

Let us show that $\widetilde{\Xi}$ does not have critical points in $\widetilde{\Omega}$. Indeed, suppose that $\widehat{z}$ is a critical point of $\widetilde{\Xi}$. Then $\widehat{z}$ is a critical point of $\Phi_{2}$. Consider the perturbation $\Phi_{1}+\epsilon \Psi_{1}$, where $\Psi_{1}$ is a smooth holomorphic function in $\chi_{1}(\widetilde{\Omega})$ such that $\left.\operatorname{Im} \Psi_{1}\right|_{\chi_{1}\left(\Gamma_{0}\right)}=0$ and for the function $\Psi_{2}$ given by (6.11) we have $\partial_{z} \Psi_{2}(\widehat{z}) \neq 0$. The mapping $\widetilde{\Xi}$ is still the same but the critical points for $\Phi_{2}+\epsilon \Psi_{2}$ change, which is a contradiction.

Let us show that $\widetilde{\Xi}$ is a single-valued function. Indeed, suppose that $\widetilde{\Xi}$ is multivalued around some point $\widetilde{z}$. Then there exists a holomorphic function $\Phi_{1}$ such that $\partial_{z} \Phi_{1}(\widetilde{z})=0$ and $\Phi_{2}=\Phi_{1} \circ \widetilde{\Xi}$. Obviously

$$
\{z \mid z=\widetilde{\Xi}(\widehat{z})\} \subset\left\{z \mid \partial_{z} \Phi_{1}=0\right\} .
$$

Let $\Psi_{1}$ be a function holomorphic in $\mathcal{O}$ and smooth on $\overline{\mathcal{O}}$ such that $\left.\operatorname{Im} \Psi_{1}\right|_{\chi_{1}\left(\Gamma_{0}\right) \cup \Pi}$ $=0$ and $\partial_{z} \Psi_{1} \neq 0$ for all $z \in\left\{z \mid \partial_{z} \Phi_{1}=0\right\}$. Then for the function $\Phi_{1}+\epsilon \Psi_{1}$ we should again have $\widetilde{\Xi}(\widehat{z}) \in\left\{z \mid \partial_{z}\left(\Phi_{1}+\epsilon \Psi_{1}\right)=0\right\}$. This contradicts (6.12).

If $\widetilde{\Xi}(\widetilde{\Omega}) \neq \chi_{1}(\widetilde{\Omega})$, then we still have $\widetilde{\Xi}(\widetilde{\Omega}) \subset \chi_{1}(\widetilde{\Omega})$. On the other hand, on the boundary of $\chi_{1}(\widetilde{\Omega}) \backslash \widetilde{\Xi}(\widetilde{\Omega})$ the imaginary part of the function $\Phi_{1}$ is zero. This is impossible.

In the domain $\Omega$ consider the new infinitesimal coordinates for the operator $P_{1}$ given by the mapping $\widetilde{\Xi}^{-1} \circ \Xi(x)$. In these coordinates, the operator $P_{1}(x, D)$ has the form

$$
\widetilde{Q}(x, D)=\frac{1}{\widetilde{\mu}_{1}} \Delta+2 \widetilde{A}_{1} \frac{\partial}{\partial z}+2 \widetilde{B}_{1} \frac{\partial}{\partial \bar{z}}+\widetilde{q}_{1}
$$

Since $\widetilde{\Xi}^{-1} \circ \Xi \widetilde{\Gamma}_{\Gamma}=\mathrm{Id}$, the Cauchy data for the operators $L_{2}$ and $\widetilde{Q}$ are exactly the same. The operators $L_{2}$ and $\widetilde{Q}$ are particular cases of the operator (1.1). Since $\left.\left(\mu_{2}-\widetilde{\mu}_{1}\right)\right|_{\widetilde{\Gamma}}=0$, the partial Cauchy data $\mathcal{C}_{\mu_{2} I, A_{2}, B_{2}, q_{2}}$ and $\mathcal{C}_{\widetilde{\mu}_{1} I, \widetilde{A}_{1}, \widetilde{B}_{1}, \widetilde{q}_{1}}$ are equal. We multiply the operator $\widetilde{Q}$ by the function $\widetilde{\mu}_{1} / \mu_{2}$ and denote the resulting operator by $\widehat{Q}(x, D)$. Then by Corollary 1.1 there exists a function $\eta$ which satisfies (1.5) such that $L_{2}(x, D)=e^{-\eta} \widehat{Q}(x, D) e^{\eta}$.

Proof of Theorem 1.2. First we observe that in order to prove this theorem it suffices to prove it in the case when instead of the whole $\widetilde{\Gamma}$, both the input and output are measured on an arbitrary small neighborhood of the point $\widehat{x}$.

Now one can consider only the case when $\widetilde{\Gamma} \subset\left\{x_{1}=0\right\}$ is a small neighborhood of the point 0 . We observe that if

$$
\left.\left(\sigma_{1}^{22}-\sigma_{2}^{22}\right)\right|_{\widetilde{\Gamma}}=\left.\frac{\partial}{\partial x_{2}}\left(\sigma_{1}^{22}-\sigma_{2}^{22}\right)\right|_{\widetilde{\Gamma}}=0,
$$


then an argument similar to that in [20] and [27] yields

$$
\sigma_{1}=\sigma_{2} \quad \text { on } \widetilde{\Gamma}, \quad \frac{\partial \sigma_{1}}{\partial x_{2}}=\frac{\partial \sigma_{2}}{\partial x_{2}} \quad \text { on } \widetilde{\Gamma} .
$$

Let us show that there exists a diffeomorphism

$$
F: \Omega \rightarrow \Omega, \quad F(x)=x, \quad \forall x \in \widetilde{\Gamma},
$$

such that for the metric $\widetilde{\sigma}_{1}=\left|\operatorname{det} D F^{-1}\right| F^{*} \sigma_{1}$ we have

$$
\widetilde{\sigma}_{1}^{22}=\sigma_{2}^{22} \quad \text { on } \widetilde{\Gamma} .
$$

First assume that we already have

$$
\sigma_{1}^{22}(0)=\sigma_{2}^{22}(0) \quad \text { and } \quad \frac{\partial}{\partial x_{2}}\left(\sigma_{1}^{22}-\sigma_{2}^{22}\right)(0)=0 .
$$

Let $y=y(x)$ be some diffeomorphism of $\Omega$ into itself. By $x=x(y)$ we denote the inverse mapping. Then

$$
\tilde{\sigma}_{1}^{22}=\sigma_{1}^{11}\left(\frac{\partial x_{1}}{\partial y_{2}}\right)^{2} / \frac{\partial x_{2}}{\partial y_{2}}+2 \sigma_{1}^{12} \frac{\partial x_{1}}{\partial y_{2}}+\sigma_{1}^{22} \frac{\partial x_{2}}{\partial y_{2}} .
$$

This equality and (6.17) immediately imply that taking a perturbation of the identity mapping one can construct a diffeomorphism of $\Omega$ into itself which satisfies (6.14) and (6.16).

Let us construct a diffeomorphism which satisfies (6.14) such that (6.17) holds true. Let $\rho$ be a smooth function such that $\left.\rho\right|_{\partial \Omega}=0$ and $\rho$ is strictly positive in $\Omega$ and $\left.\frac{\partial \rho}{\partial \nu}\right|_{\partial \Omega}<0$. Consider the system of ordinary differential equations

$$
\frac{d y_{1}}{d t}=\rho(y) f_{1}(y), \quad \frac{d y_{2}}{d t}=\rho(y) f_{2}(y) .
$$

The corresponding phase flow $g^{s}$ is a diffeomorphism of $\Omega$ into itself such that $g^{s}(x)=x$ on $\partial \Omega$. Let $f_{1}(0) \neq 0$ and $f_{2}(0) \neq 0$. With appropriate choice of $f_{1}, f_{2}$, one can arrange that $\left(\partial x_{1} / \partial y_{2}\right)(0)=0$ and $\left(\partial x_{2} / \partial y_{2}\right)(0)=\sigma_{2}^{22}(0) / \sigma_{1}^{22}(0)$. Then the first equality in (6.17) holds true. Adjusting the second derivatives of $f_{1}, f_{2}$, we can arrange that

$$
\frac{\partial^{2} x_{1}}{\partial y_{2}^{2}}(0)=0 \quad \text { and } \quad \frac{\partial^{2} x_{2}}{\partial y_{2}^{2}}(0)=\frac{1}{\sigma_{1}^{22}(0)}\left(\frac{\partial \sigma_{2}^{22}}{\partial y_{2}}(0)-\frac{\partial \sigma_{1}^{22}}{\partial y_{2}}(0) \frac{\partial x_{2}}{\partial y_{2}}(0)\right) .
$$

Then the second equality in (6.17) holds true.

Now (6.15) is established. The rest of the proof of Theorem 1.2 is similar to the proof of Theorem 1.1. 


\section{$\S 7$. Appendix I}

Consider the following problem for the Cauchy-Riemann equations:

$$
\begin{aligned}
& L(\phi, \psi)=\left(\frac{\partial \phi}{\partial x_{1}}-\frac{\partial \psi}{\partial x_{2}}, \frac{\partial \phi}{\partial x_{2}}+\frac{\partial \psi}{\partial x_{1}}\right)=0 \quad \text { in } \Omega, \\
& \left.(\phi, \psi)\right|_{\Gamma_{0}}=\left(b_{1}(x), b_{2}(x)\right), \\
& \frac{\partial^{l}}{\partial z^{l}}(\phi+i \psi)\left(\widehat{x}_{j}\right)=c_{0, j} \quad \forall j \in\{1, \ldots, N\} \text { and } \forall l \in\{0, \ldots, 5\} .
\end{aligned}
$$

Here $\widehat{x}_{1}, \ldots, \widehat{x}_{N}$ are arbitrary fixed points in $\Omega$. We consider real-valued functions $b_{1}, b_{2}$ and complex numbers $\vec{C}=\left(c_{0,1}, c_{1,1}, c_{2,1}, c_{3,1}, c_{4,1}, c_{5,1}, \ldots, c_{0, N}, c_{1, N}, c_{2, N}\right.$, $\left.c_{3, N}, c_{4, N}, c_{5, N}\right)$ as initial data for (7.1). The following proposition establishes the solvability of (7.1) for a dense set of Cauchy data.

Proposition 7.1. There exists a dense subset $\mathcal{O} \subset C^{6}\left(\overline{\Gamma_{0}}\right) \times C^{6}\left(\overline{\Gamma_{0}}\right) \times \mathbb{C}^{6 N}$ such that for each $\left(b_{1}, b_{2}, \vec{C}\right) \in \mathcal{O},(7.1)$ has at least one solution $(\phi, \psi) \in C^{6}(\bar{\Omega}) \times C^{6}(\bar{\Omega})$.

Proof. Denote by $B=\left(b_{1}, b_{2}\right)$ an arbitrary element of $C^{7}\left(\overline{\Gamma_{0}}\right) \times C^{7}\left(\overline{\Gamma_{0}}\right)$. Consider the extremal problem

$$
\begin{aligned}
J_{\epsilon}(\phi, \psi) & =\|(\phi, \psi)-B\|_{B_{4}^{27 / 4}\left(\Gamma_{0}\right)}^{4}+\epsilon \sum_{k=0}^{3}\left\|\frac{\partial^{k}(\phi, \psi)}{\partial \nu^{k}}\right\|_{B_{4}^{27 / 4-k}(\partial \Omega)}^{4} \\
& +\frac{1}{\epsilon}\left\|\Delta^{3} L(\phi, \psi)\right\|_{L^{4}(\Omega)}^{4}+\sum_{j=1}^{N} \sum_{k=0}^{5}\left|\frac{\partial^{k}}{\partial z^{k}}(\phi+i \psi)\left(\widehat{x}_{j}\right)-c_{k, j}\right|^{2} \rightarrow \inf , \\
(\phi, \psi) \in & W_{4}^{7}(\Omega) \times W_{4}^{7}(\Omega) .
\end{aligned}
$$

Here $B_{k}^{l}$ denotes the Besov space of the corresponding orders.

For each $\epsilon>0$ there exists a unique solution to (7.2), (7.3), which we denote by $\left(\widehat{\phi}_{\epsilon}, \widehat{\psi}_{\epsilon}\right)$. This fact can be proved by standard arguments. We fix $\epsilon>0$. Denote by $\mathcal{U}_{\text {ad }}$ the set of admissible elements for the problem (7.2), (7.3), namely

$$
\mathcal{U}_{\mathrm{ad}}=\left\{(\phi, \psi) \in W_{4}^{7}(\Omega) \times W_{4}^{7}(\Omega) \mid J_{\epsilon}(\phi, \psi)<\infty\right\} .
$$

Denote $\widehat{J}_{\epsilon}=\inf _{(\phi, \psi) \in W_{4}^{7}(\Omega) \times W_{4}^{7}(\Omega)} J_{\epsilon}(\phi, \psi)$. Clearly $(0,0) \in \mathcal{U}_{\text {ad }}$. Therefore there exists a minimizing sequence $\left\{\left(\phi_{k}, \psi_{k}\right)\right\}_{k=1}^{\infty} \subset W_{4}^{7}(\Omega) \times W_{4}^{7}(\Omega)$ such that

$$
\widehat{J}_{\epsilon}=\lim _{k \rightarrow \infty} J_{\epsilon}\left(\phi_{k}, \psi_{k}\right) \text {. }
$$

Observe that the minimizing sequence is bounded in $W_{4}^{7}(\Omega) \times W_{4}^{7}(\Omega)$. Indeed, since the sequence $\left\{\Delta^{3} L\left(\phi_{k}, \psi_{k}\right),\left.L\left(\phi_{k}, \psi_{k}\right)\right|_{\partial \Omega}, \ldots,\left.\frac{\partial^{3}}{\partial \nu^{3}} L\left(\phi_{k}, \psi_{k}\right)\right|_{\partial \Omega}\right\}$ is bounded in $L^{4}(\Omega) \times L^{4}(\Omega) \times \prod_{k=0}^{3} B_{4}^{27 / 4-k}(\partial \Omega) \times B_{4}^{27 / 4-k}(\partial \Omega)$, the standard elliptic $L^{p_{-}}$ estimate implies that the sequence $\left\{L\left(\phi_{k}, \psi_{k}\right)\right\}$ is bounded in $W_{4}^{6}(\Omega) \times W_{4}^{6}(\Omega)$. 
Taking into account that the sequence of the traces of the functions $\left(\phi_{k}, \psi_{k}\right)$ is bounded in $B_{4}^{27 / 4}(\partial \Omega) \times B_{4}^{27 / 4}(\partial \Omega)$ and applying the estimates for elliptic operators one more time, we find that $\left\{\left(\phi_{k}, \psi_{k}\right)\right\}$ is bounded in $W_{4}^{7}(\Omega) \times W_{4}^{7}(\Omega)$. By the Sobolev imbedding theorem, the sequence $\left\{\left(\phi_{k}, \psi_{k}\right)\right\}$ is bounded in $C^{6}(\bar{\Omega}) \times C^{6}(\bar{\Omega})$. Then taking a subsequence if necessary (denoted again by $\left\{\left(\phi_{k}, \psi_{k}\right)\right\}$ ), we obtain

$$
\begin{aligned}
& \left(\phi_{k}, \psi_{k}\right) \rightarrow\left(\widehat{\phi}_{\epsilon}, \widehat{\psi}_{\epsilon}\right) \quad \text { weakly in } W_{4}^{7}(\Omega) \times W_{4}^{7}(\Omega), \\
& \left(\frac{\partial^{j} \phi_{k}}{\partial \nu^{j}}, \frac{\partial^{j} \psi_{k}}{\partial \nu^{j}}\right) \rightarrow\left(\frac{\partial^{j} \widehat{\phi}_{\epsilon}}{\partial \nu^{j}}, \frac{\partial^{j} \widehat{\psi}_{\epsilon}}{\partial \nu^{j}}\right) \quad \text { weakly in } B_{4}^{27 / 4-j}(\partial \Omega) \times B_{4}^{27 / 4-j}(\partial \Omega) \\
& \frac{\partial^{k}}{\partial z^{k}}(\phi+i \psi)\left(\widehat{x}_{j}\right)-c_{k, j} \rightarrow C_{k, j, \epsilon}, \quad k \in\{0, \ldots, 5\}, \\
& \left.\Delta^{3} L\left(\phi_{k}, \psi_{k}\right) \rightarrow r_{\epsilon} \quad \text { weakly in } L^{4}(\Omega) \times L^{4}(\Omega), 2,3\right\}, \\
& L\left(\phi_{k}, \psi_{k}\right) \rightarrow \widetilde{r}_{\epsilon} \quad \text { weakly in } W_{4}^{6}(\Omega) \times W_{4}^{6}(\Omega) .
\end{aligned}
$$

Obviously, $r_{\epsilon}=\Delta^{3} L\left(\widehat{\phi}_{\epsilon}, \widehat{\psi}_{\epsilon}\right)$ and $\widetilde{r}_{\epsilon}=L\left(\widehat{\phi}_{\epsilon}, \widehat{\psi}_{\epsilon}\right)$. Then, since the norms in $L^{4}(\Omega)$ and $B_{4}^{27 / 4-k}(\partial \Omega)$ are lower semicontinuous with respect to weak convergence, we obtain

$$
J_{\epsilon}\left(\widehat{\phi}_{\epsilon}, \widehat{\psi}_{\epsilon}\right) \leq \lim _{k \rightarrow \infty} J_{\epsilon}\left(\phi_{k}, \psi_{k}\right)=\widehat{J}_{\epsilon}
$$

Thus the pair $\left(\widehat{\phi}_{\epsilon}, \widehat{\psi}_{\epsilon}\right)$ is a solution to the extremal problem (7.2), (7.3). Since the set of admissible elements is convex and the functional $J_{\epsilon}$ is strictly convex, this solution is unique.

By Fermat's theorem we have

$$
J_{\epsilon}^{\prime}\left(\widehat{\phi}_{\epsilon}, \widehat{\psi}_{\epsilon}\right)[\widetilde{\delta}]=0 \quad \forall \widetilde{\delta} \in W_{4}^{7}(\Omega) \times W_{4}^{7}(\Omega) .
$$

This equality can be written in the form

$$
\begin{aligned}
& I_{\Gamma_{0}, 27 / 4}^{\prime}\left(\left(\widehat{\phi}_{\epsilon}, \widehat{\psi}_{\epsilon}\right)-B\right)[\widetilde{\delta}]+\epsilon \sum_{k=0}^{3} I_{\partial \Omega, 27 / 4-k}^{\prime}\left(\frac{\partial^{k}}{\partial \nu^{k}}\left(\widehat{\phi}_{\epsilon}, \widehat{\psi}_{\epsilon}\right)\right)\left[\frac{\partial^{k}}{\partial \nu^{k}} \widetilde{\delta}\right] \\
& +\left(p_{\epsilon}, \Delta^{3} L \widetilde{\delta}\right)_{L^{2}(\Omega)}+\sum_{j=1}^{N} \sum_{k=0}^{5}\left(\frac{\partial^{k}}{\partial z^{k}}\left(\widehat{\phi}_{\epsilon}+i \widehat{\psi}_{\epsilon}\right)\left(\widehat{x}_{j}\right)-c_{k, j}\right) \overline{\frac{\partial^{k}}{\partial z^{k}}\left(\widetilde{\delta}_{1}+i \widetilde{\delta}_{2}\right)}\left(\widehat{x}_{j}\right) \\
& +\overline{\left(\frac{\partial^{k}}{\partial z^{k}}\left(\widehat{\phi}_{\epsilon}+i \widehat{\psi}_{\epsilon}\right)\left(\widehat{x}_{j}\right)-c_{k, j}\right)} \frac{\partial^{k}}{\partial z^{k}}\left(\widetilde{\delta}_{1}+i \widetilde{\delta}_{2}\right)\left(\widehat{x}_{j}\right)=0,
\end{aligned}
$$

where

$$
p_{\epsilon}=\frac{4}{\epsilon}\left(\left(\Delta^{3}\left(\frac{\partial \widehat{\phi}_{\epsilon}}{\partial x_{1}}-\frac{\partial \widehat{\psi}_{\epsilon}}{\partial x_{2}}\right)\right)^{3},\left(\Delta^{3}\left(\frac{\partial \widehat{\phi}_{\epsilon}}{\partial x_{2}}+\frac{\partial \widehat{\psi}_{\epsilon}}{\partial x_{1}}\right)\right)^{3}\right)
$$

and $I_{\Gamma^{*}, \kappa}^{\prime}(\widehat{w})$ denotes the derivative of the functional $w \mapsto\|w\|_{B_{4}^{\kappa}\left(\Gamma^{*}\right)}^{4}$ at $\widehat{w}$. 
Observe that we have $J_{\epsilon}\left(\widehat{\phi}_{\epsilon}, \widehat{\psi}_{\epsilon}\right) \leq J_{\epsilon}(0,0)=\|B\|_{B_{4}^{27 / 4}\left(\Gamma_{0}\right)}^{4}+\sum_{j=1}^{N} \sum_{k=0}^{5}\left|c_{k, j}\right|^{2}$. This implies that the sequence $\left\{\left(\widehat{\phi}_{\epsilon}, \widehat{\psi}_{\epsilon}\right)\right\}$ is bounded in $B_{4}^{27 / 4}\left(\Gamma_{0}\right) \times B_{4}^{27 / 4}\left(\Gamma_{0}\right)$, the sequences $\left\{\frac{\partial^{k}}{\partial z^{k}}\left(\widehat{\phi}_{\epsilon}+i \widehat{\psi}_{\epsilon}\right)\left(\widehat{x}_{j}\right)-c_{k, j}\right\}$ are bounded in $\mathbb{C}$ and the sequence $\epsilon \sum_{k=0}^{3} I_{\partial \Omega, 27 / 4-k}^{\prime}\left(\frac{\partial^{k}}{\partial \nu^{k}}\left(\widehat{\phi}_{\epsilon}, \widehat{\psi}_{\epsilon}\right)\right)\left[\frac{\partial^{k}}{\partial \nu^{k}} \widetilde{\delta}\right]$ converges to zero for any $\widetilde{\delta}$ from $B_{4}^{27 / 4}(\partial \Omega)$ $\times B_{4}^{27 / 4}(\partial \Omega)$. Then (7.4) implies that the sequence $\left\{p_{\epsilon}\right\}_{\epsilon \in(0,1)}$ is bounded in $L^{4 / 3}(\Omega)$ $\times L^{4 / 3}(\Omega)$.

Therefore there exist $\mathcal{B} \in B_{4}^{27 / 4}\left(\Gamma_{0}\right) \times B_{4}^{27 / 4}\left(\Gamma_{0}\right), C_{0, j}, C_{1, j}, \ldots, C_{5, j} \in \mathbb{C}$ and $p=\left(p_{1}, p_{2}\right) \in L^{4 / 3}(\Omega) \times L^{4 / 3}(\Omega)$ such that

$$
\begin{aligned}
& \left(\widehat{\phi}_{\epsilon}, \widehat{\psi}_{\epsilon}\right)-B \rightarrow \mathcal{B} \quad \text { weakly in } B_{4}^{27 / 4}\left(\Gamma_{0}\right) \times B_{4}^{27 / 4}\left(\Gamma_{0}\right), \\
& p_{\epsilon} \rightarrow p \quad \text { weakly in } L^{4 / 3}(\Omega) \times L^{4 / 3}(\Omega), \\
& \frac{\partial^{k}}{\partial z^{k}}\left(\widehat{\phi}_{\epsilon}+i \widehat{\psi}_{\epsilon}\right)\left(\widehat{x}_{j}\right)-c_{k, j} \rightarrow C_{k, j}, \quad k \in\{0,1, \ldots, 5\}, j \in\{1, \ldots, N\} .
\end{aligned}
$$

Passing to the limit in (7.4) we obtain

$$
\begin{aligned}
& I_{\Gamma_{0}, 27 / 4}^{\prime}(\mathcal{B})[\widetilde{\delta}]+\left(p, \Delta^{3} L \widetilde{\delta}\right)_{L^{2}(\Omega)} \\
& +2 \operatorname{Re} \sum_{j=1}^{N} \sum_{k=0}^{5} C_{k, j} \overline{\frac{\partial^{k}}{\partial z^{k}}\left(\widetilde{\delta}_{1}+i \widetilde{\delta}_{2}\right)}\left(\widehat{x}_{j}\right)=0 \quad \forall \widetilde{\delta} \in W_{4}^{7}(\Omega) \times W_{4}^{7}(\Omega) .
\end{aligned}
$$

Next we claim that

$$
\Delta^{3} p=0 \quad \text { in } \Omega \backslash \bigcup_{j=1}^{N}\left\{\widehat{x}_{j}\right\}
$$

in the sense of distributions. Suppose that (7.8) is already proved. This implies

$$
\left(p, \Delta^{3} L \widetilde{\delta}\right)_{L^{2}(\Omega)}+2 \operatorname{Re} \sum_{j=1}^{N} \sum_{k=0}^{5} C_{k, j} \overline{\frac{\partial^{k}}{\partial z^{k}}\left(\widetilde{\delta}_{1}+i \widetilde{\delta}_{2}\right)}\left(\widehat{x}_{j}\right)=0 \quad \forall \widetilde{\delta}_{1}, \widetilde{\delta}_{2} \in C_{0}^{\infty}(\Omega)
$$

If $p=\left(p_{1}, p_{2}\right)$, then denoting $P=p_{1}-i p_{2}$, we have

$$
\begin{aligned}
& \operatorname{Re}\left(\Delta^{3} P, \partial_{\bar{z}}\left(\widetilde{\delta}_{1}+i \widetilde{\delta}_{2}\right)\right)_{L^{2}(\Omega)} \\
& \quad+\operatorname{Re} \sum_{j=1}^{N} \sum_{k=0}^{5} \overline{C_{k, j}} \frac{\partial^{k}}{\partial z^{k}}\left(\widetilde{\delta}_{1}+i \widetilde{\delta}_{2}\right)\left(\widehat{x}_{j}\right)=0 \quad \forall \widetilde{\delta}_{1}, \widetilde{\delta}_{2} \in C_{0}^{\infty}(\Omega) .
\end{aligned}
$$

Since supp $\Delta^{3} P \subset \bigcup_{j=1}^{N}\left\{\widehat{x}_{j}\right\}$ by (7.8), there exist some constants $m_{\beta, j}$ and $\widehat{\ell}_{j}$ such that $\Delta^{3} P=\sum_{j=1}^{N} \sum_{|\beta|=1}^{\widehat{\ell}} m_{\beta, j} D^{\beta} \delta\left(x-\widehat{x}_{j}\right)$. The above equality can be written in 
the form

$$
-\sum_{|\beta|=1}^{\widehat{\ell}_{j}} m_{\beta, j} \frac{\partial}{\partial \bar{z}} D^{\beta} \delta\left(x-\widehat{x}_{j}\right)=\sum_{k=0}^{5}(-1)^{k} \overline{C_{k, j}} \frac{\partial^{k}}{\partial z^{k}} \delta\left(x-\widehat{x}_{j}\right)
$$

From this we obtain

$$
C_{0, j}=C_{1, j}=\cdots=C_{5, j}=0, \quad j \in\{1, \ldots, N\} .
$$

Therefore

$$
\Delta^{3} p=0 \quad \text { in } \Omega
$$

This implies

$$
\begin{gathered}
\left(p, \Delta^{3} L \widetilde{\delta}\right)_{L^{2}(\Omega)}=0 \quad \forall \widetilde{\delta} \in W_{4}^{7}(\Omega) \times W_{4}^{7}(\Omega), \\
\left.L \widetilde{\delta}\right|_{\partial \Omega}=\left.\frac{\partial L \widetilde{\delta}}{\partial \nu}\right|_{\partial \Omega}=\cdots=\left.\frac{\partial^{5} L \widetilde{\delta}}{\partial \nu^{5}}\right|_{\partial \Omega}=0 .
\end{gathered}
$$

This equality and (7.7) yield

$$
\begin{gathered}
I_{\Gamma_{0}, 27 / 4}^{\prime}(\mathcal{B})[\widetilde{\delta}]=0 \quad \forall \widetilde{\delta} \in W_{4}^{7}(\Omega) \times W_{4}^{7}(\Omega), \\
\left.L \widetilde{\delta}\right|_{\partial \Omega}=\left.\frac{\partial L \widetilde{\delta}}{\partial \nu}\right|_{\partial \Omega}=\cdots=\left.\frac{\partial^{5} L \widetilde{\delta}}{\partial \nu^{5}}\right|_{\partial \Omega}=0 .
\end{gathered}
$$

Then using the trace theorem, we conclude that $\mathcal{B}=0$. Using this and (7.5), we obtain

$$
\left(\widehat{\phi}_{\epsilon_{k}}, \widehat{\psi}_{\epsilon_{k}}\right)-B \rightarrow 0 \text { weakly in } B_{4}^{27 / 4}\left(\Gamma_{0}\right) \times B_{4}^{27 / 4}\left(\Gamma_{0}\right)
$$

From (7.6) and (7.9), we obtain

$$
\frac{\partial^{k}}{\partial z^{k}}\left(\widehat{\phi}_{\epsilon}+i \widehat{\psi}_{\epsilon}\right)(\widehat{x}) \rightarrow c_{k, j}, \quad k \in\{0,1, \ldots, 5\}, j \in\{1, \ldots, N\} .
$$

The Sobolev embedding theorem yields $B_{4}^{27 / 4}\left(\Gamma_{0}\right) \subset \subset C^{5}\left(\overline{\Gamma_{0}}\right)$. Therefore $(7.12)$ implies

$$
\left(\widehat{\phi}_{\epsilon_{k}}, \widehat{\psi}_{\epsilon_{k}}\right)-B \rightarrow 0 \quad \text { in } C^{5}\left(\overline{\Gamma_{0}}\right) \times C^{5}\left(\overline{\Gamma_{0}}\right) .
$$

Let the pair $\left(\widetilde{\phi}_{\epsilon_{k}}, \widetilde{\psi}_{\epsilon_{k}}\right)$ be a solution to the boundary value problem

$$
L\left(\widetilde{\phi}_{\epsilon_{k}}, \widetilde{\psi}_{\epsilon_{k}}\right)=L\left(\widehat{\phi}_{\epsilon_{k}}, \widehat{\psi}_{\epsilon_{k}}\right) \quad \text { in } \Omega,\left.\quad \widetilde{\psi}_{\epsilon_{k}}\right|_{\partial \Omega}=\psi_{\epsilon_{k}}^{*} .
$$

Here $\psi_{\epsilon_{k}}^{*}$ is a smooth function such that $\left.\psi_{\epsilon_{k}}^{*}\right|_{\Gamma_{0}}=0$ and the pair $\left(L\left(\widehat{\phi}_{\epsilon_{k}}, \widehat{\psi}_{\epsilon_{k}}\right), \psi_{\epsilon_{k}}^{*}\right)$ is orthogonal to all solutions of the adjoint problem (see [29]). Moreover since $L\left(\widetilde{\phi}_{\epsilon_{k}}, \widetilde{\psi}_{\epsilon_{k}}\right) \rightarrow 0$ in $W_{4}^{6}(\Omega) \times W_{4}^{6}(\Omega)$ we may assume $\psi_{\epsilon_{k}}^{*} \rightarrow 0$ in $C^{6}(\partial \Omega) \times C^{6}(\partial \Omega)$. 
Among all possible solutions to problem (7.14) (clearly there is no uniqueness of solution for this problem), we choose one such that $\int_{\Omega} \widetilde{\phi}_{\epsilon_{k}} d x=0$. Thus we obtain

$$
\left(\widetilde{\phi}_{\epsilon_{k}}, \widetilde{\psi}_{\epsilon_{k}}\right) \rightarrow 0 \quad \text { in } W_{4}^{7}(\Omega) \times W_{4}^{7}(\Omega) .
$$

Therefore $\left\{\left(\widehat{\phi}_{\epsilon_{k}}-\widetilde{\phi}_{\epsilon_{k}}, \widehat{\psi}_{\epsilon_{k}}-\widetilde{\psi}_{\epsilon_{k}}\right)\right\}$ is the desired approximation to the solution of the Cauchy problem (7.1).

Now we prove (7.8). Let $\widetilde{x} \in \Omega \backslash \bigcup_{j=1}^{N}\left\{\widehat{x}_{j}\right\}$, let $\widetilde{\chi}$ be a smooth function which is zero in some neighborhood of $\Gamma_{0} \cup \bigcup_{j=1}^{N}\left\{\widehat{x}_{j}\right\}$ and suppose $\mathcal{D}=\{x \in \Omega \mid \widetilde{\chi}(x)=1\}$ contains an open connected subset $\mathcal{F}$ such that $\widetilde{x} \in \mathcal{F}$ and $\widetilde{\Gamma} \cap \overline{\mathcal{F}}$ is an open set in $\partial \Omega$. In addition we assume that $\operatorname{Int}(\operatorname{supp} \chi)$ is a simply connected domain. By (7.7) we have

$$
\begin{aligned}
0 & =\left(p, \Delta^{3} L(\widetilde{\chi} \widetilde{\delta})\right)_{L^{2}(\Omega)} \\
& =\left(\widetilde{\chi} p, \Delta^{3} L \widetilde{\delta}\right)_{L^{2}(\Omega)}+\left(p,\left[\Delta^{3} L, \widetilde{\chi}\right] \widetilde{\delta}\right)_{L^{2}(\Omega)} \quad \forall \widetilde{\delta} \in W_{4}^{7}(\Omega) \times W_{4}^{7}(\Omega) .
\end{aligned}
$$

Consider the linear functional mapping $\widehat{\delta} \in W_{4}^{2}(\operatorname{supp} \widetilde{\chi}) \times W_{4}^{2}(\operatorname{supp} \widetilde{\chi})$ to $\left(p,\left[\Delta^{3} L, \widetilde{\chi}\right] \widetilde{\delta}\right)_{L^{2}(\Omega)}$, where

$$
L \widetilde{\delta}=\widehat{\delta} \quad \text { in } \Omega,\left.\quad \operatorname{Im} \widetilde{\delta}\right|_{\mathcal{S}}=0, \quad \int_{\operatorname{supp} \tilde{\chi}} \operatorname{Re} \widetilde{\delta} d x=0,
$$

where $\mathcal{S}$ denotes the boundary of supp $\widetilde{\chi}$. For each $\widehat{\delta} \in W_{4}^{2}(\operatorname{supp} \widetilde{\chi}) \times W_{4}^{2}(\operatorname{supp} \widetilde{\chi})$, there exists a unique solution $\widetilde{\delta} \in W_{4}^{3}(\operatorname{supp} \widetilde{\chi}) \times W_{4}^{3}(\operatorname{supp} \widetilde{\chi})$. Hence the functional is well-defined and continuous on $W_{4}^{2}(\operatorname{supp} \tilde{\chi})$. Therefore there exist $\mathbf{q}, \mathbf{r}, q_{0} \in L^{4 / 3}(\operatorname{supp} \tilde{\chi})$ such that $\int_{\operatorname{supp} \tilde{\chi}}\left(\sum_{j, k=1}^{2} r_{j k} \frac{\partial^{2}}{\partial x_{j} \partial x_{k}} \widehat{\delta}+(\mathbf{q}, \widehat{\delta})+q_{0} \widehat{\delta}\right) d x=$ $\left(p,\left[\Delta^{3} L, \widetilde{\chi}\right] \widetilde{\delta}\right)_{L^{2}(\operatorname{supp} \tilde{\chi})}$.

Consider the boundary value problem

$$
\Delta^{3} \widetilde{P}=\widetilde{f} \quad \operatorname{in} \operatorname{supp} \chi,\left.\quad \widetilde{P}\right|_{\mathcal{S}}=\left.\frac{\partial \widetilde{P}}{\partial \nu}\right|_{\mathcal{S}}=\left.\frac{\partial^{2} \widetilde{P}}{\partial \nu^{2}}\right|_{\mathcal{S}}=0 .
$$

Here $\widetilde{f}=2 \operatorname{div}(\nabla \widetilde{\mathbf{q}})-q_{0}-\sum_{j, k=1}^{2} \frac{\partial^{2}}{\partial x_{j} \partial x_{k}} r_{j k}$. A solution to this problem exists and is unique, since $\tilde{f} \in\left(\stackrel{\circ}{W}_{4}^{2}(\operatorname{supp} \tilde{\chi})\right)^{\prime}$. Then $P \in \stackrel{\circ}{W}_{4 / 3}^{1}(\operatorname{supp} \widetilde{\chi}) \times \stackrel{\circ}{W}_{4 / 3}^{1}(\operatorname{supp} \widetilde{\chi})$. On the other hand, thanks to $(7.16), P=\widetilde{\chi} p \in \stackrel{\circ}{W}_{4 / 3}^{1}(\operatorname{supp} \tilde{\chi}) \times \stackrel{\circ}{W}_{4 / 3}^{1}(\operatorname{supp} \tilde{\chi})$.

Next we take another smooth cut-off function $\widetilde{\chi}_{1}$ such that $\operatorname{supp} \widetilde{\chi}_{1} \subset \mathcal{D}$ and $\operatorname{Int}\left(\operatorname{supp} \chi_{1}\right)$ is a simply connected domain. A neighborhood of $\widetilde{x}$ lies in $\mathcal{D}_{1}=\{x \mid$ $\left.\tilde{\chi}_{1}=1\right\}$, the interior of $\mathcal{D}_{1}$ is connected, and $\overline{\operatorname{Int} \mathcal{D}_{1}} \cap \widetilde{\Gamma}$ contains an open subset $\mathcal{O}$ in $\partial \Omega$. Similarly to (7.16) we have

$$
\left(\widetilde{\chi}_{1} p, \Delta^{3} L \widetilde{\delta}\right)_{L^{2}(\Omega)}-\left(p,\left[\Delta^{3} L, \widetilde{\chi}_{1}\right] \widetilde{\delta}\right)_{L^{2}(\Omega)}=0 \quad \forall \widetilde{\delta} \in W_{4}^{7}(\Omega) \times W_{4}^{7}(\Omega) .
$$

This implies that $\tilde{\chi}_{1} p \in W_{4 / 3}^{2}(\Omega) \times W_{4 / 3}^{2}(\Omega)$, by a similar argument to the one above. 
Next we take another smooth cut-off function $\widetilde{\chi}_{2}$ such that $\operatorname{supp} \widetilde{\chi}_{2} \subset \mathcal{D}_{1}$, $\operatorname{Int}\left(\operatorname{supp} \chi_{2}\right)$ is a simply connected domain, a neighborhood of $\widetilde{x}$ is contained in $\mathcal{D}_{2}=\left\{x \mid \widetilde{\chi}_{2}=1\right\}$, the interior of $\mathcal{D}_{2}$ is connected, and $\overline{\operatorname{Int} \mathcal{D}_{2}} \cap \widetilde{\Gamma}$ contains an open subset $\mathcal{O}$ in $\partial \Omega$. Similarly to (7.16) we have

$$
\left(\widetilde{\chi}_{2} p, \Delta^{3} L \widetilde{\delta}\right)_{L^{2}(\Omega)}-\left(p,\left[\Delta^{3} L, \widetilde{\chi}_{2}\right] \widetilde{\delta}\right)_{L^{2}(\Omega)}=0 \quad \forall \widetilde{\delta} \in W_{4}^{7}(\Omega) \times W_{4}^{7}(\Omega) .
$$

This implies that $\tilde{\chi}_{2} p \in W_{4 / 3}^{3}(\Omega) \times W_{4 / 3}^{3}(\Omega)$, by a similar argument to the one above. Let $\omega$ be a domain such that $\omega \cap \Omega=\emptyset$ and $\partial \omega \cap \partial \Omega \subset \mathcal{O}$ contains an open set in $\partial \Omega$.

We extend $p$ onto $\omega$ by zero. Then

$$
\left(\Delta^{3}\left(\widetilde{\chi}_{2} p\right), L \widetilde{\delta}\right)_{L^{2}(\Omega \cup \omega)}+\left(p,\left[\Delta^{3} L, \widetilde{\chi}_{2}\right] \widetilde{\delta}\right)_{L^{2}(\Omega \cup \omega)}=0 .
$$

Hence, since $\left.\left[\Delta^{3} L, \widetilde{\chi}_{2}\right]\right|_{\operatorname{Int} \mathcal{D}_{2}}=0$ we have

$$
L^{*} \Delta^{3}\left(\widetilde{\chi}_{2} p\right)=0 \quad \text { in } \operatorname{Int} \mathcal{D}_{2} \cup \omega,\left.\quad p\right|_{\omega}=0 .
$$

By Holmgren's theorem $\left.\Delta^{3}\left(\widetilde{\chi}_{2} p\right)\right|_{\operatorname{Int} \mathcal{D}_{2}}=0$, that is, $\left(\Delta^{3} p\right)(\widetilde{x})=0$. Thus $(7.8)$ is proved.

Consider the Cauchy problem for the Cauchy-Riemann equations

$$
\begin{aligned}
& L(\phi, \psi)=\left(\frac{\partial \phi}{\partial x_{1}}-\frac{\partial \psi}{\partial x_{2}}, \frac{\partial \phi}{\partial x_{2}}+\frac{\partial \psi}{\partial x_{1}}\right)=0 \quad \text { in } \Omega,\left.\quad(\phi, \psi)\right|_{\Gamma_{0}}=(b(x), 0), \\
& \frac{\partial^{l}}{\partial z^{l}}(\phi+i \psi)\left(\widehat{x}_{j}\right)=c_{0, j} \quad \forall j \in\{1, \ldots, N\} \text { and } \forall l \in\{0, \ldots, 5\} .
\end{aligned}
$$

Here $\widehat{x}_{1}, \ldots, \widehat{x}_{N}$ are arbitrary fixed points in $\Omega$. We consider a real-valued function $b$ and complex numbers $\vec{C}=\left(c_{0,1}, c_{1,1}, c_{2,1}, c_{3,1}, c_{4,1}, c_{5,1}, \ldots, c_{0, N}, c_{1, N}, c_{2, N}, c_{3, N}\right.$, $\left.c_{4, N}, c_{5, N}\right)$ as initial data for (7.1). The following proposition establishes the solvability of (7.1) for a dense set of Cauchy data.

Corollary 7.1. There exists a dense subset $\mathcal{O} \subset C^{6}\left(\overline{\Gamma_{0}}\right) \times \mathbb{C}^{6 N}$ such that for each $(b, \vec{C}) \in \mathcal{O}$, problem $(7.17)$ has at least one solution $(\phi, \psi) \in C^{6}(\bar{\Omega}) \times C^{6}(\bar{\Omega})$.

The proof of Corollary 7.1 is similar to the proof of Proposition 7.1. The only difference is that instead of the extremal problem considered there, we use the following extremal problem:

$$
\begin{aligned}
& J_{\epsilon}(\phi, \psi)=\|\phi-b\|_{B_{4}^{27 / 4}\left(\Gamma_{0}\right)}^{4}+\epsilon \sum_{k=0}^{3}\left\|\frac{\partial^{k}(\phi, \psi)}{\partial \nu^{k}}\right\|_{B_{4}^{27 / 4-k}(\partial \Omega)}^{4} \\
&+\frac{1}{\epsilon}\left\|\Delta^{3} L(\phi, \psi)\right\|_{L^{4}(\Omega)}^{4}+\sum_{j=1}^{N} \sum_{k=0}^{5}\left|\frac{\partial^{k}}{\partial z^{k}}(\phi+i \psi)\left(\widehat{x}_{j}\right)-c_{k, j}\right|^{2} \rightarrow \inf , \\
&(\phi, \psi) \in W_{4}^{7}(\Omega) \times W_{4}^{7}(\Omega),\left.\quad \psi\right|_{\Gamma_{0}}=0 .
\end{aligned}
$$


We have

Proposition 7.2. Let $\alpha \in(0,1), \mathcal{A}, \mathcal{B} \in C^{6+\alpha}(\bar{\Omega})$ and let $y_{1}, \ldots, y_{\widehat{k}} \in \Gamma_{0}$, $y_{\widehat{k}+1}, \ldots, y_{\widetilde{k}} \in \Omega, \widetilde{x} \in \Omega \backslash\left\{y_{1}, \ldots, y_{\widetilde{k}}\right\}$ be arbitrary points. Then there exist $a$ holomorphic function $a \in C^{5+\alpha}(\bar{\Omega})$ and an antiholomorphic function $d \in C^{5+\alpha}(\bar{\Omega})$ such that $\left.\left(a e^{\mathcal{A}}+d e^{\mathcal{B}}\right)\right|_{\Gamma_{0}}=0$,

$$
\frac{\partial^{k+j} a}{\partial x_{1}^{k} \partial x_{2}^{j}}\left(y_{\ell}\right)=0, \quad k+j \leq 5, \ell \in\{1, \ldots, \tilde{k}\},
$$

and

$$
a(\widetilde{x}) \neq 0 \quad \text { and } \quad d(\widetilde{x}) \neq 0 .
$$

Proof. Consider the operator

$$
\begin{aligned}
R(\gamma)=\left(a\left(y_{1}\right), \ldots, \frac{\partial^{5} a}{\partial z^{5}}\left(y_{1}\right), \ldots, a\left(y_{\widetilde{k}}\right), \ldots, \frac{\partial^{5} a}{\partial z^{5}}\left(y_{\widetilde{k}}\right)\right. \\
\left.d\left(y_{1}\right), \ldots, \frac{\partial^{5} d}{\partial \bar{z}^{5}}\left(y_{1}\right), \ldots, d\left(y_{\widetilde{k}}\right), \ldots, \frac{\partial^{5} d}{\partial \bar{z}^{5}}\left(y_{\widetilde{k}}\right), a(\widetilde{x}), d(\widetilde{x})\right) .
\end{aligned}
$$

Here $\gamma \in C_{0}^{\infty}(\widetilde{\Gamma})$ and the functions $a$ and $d$ are solutions to the problem

$$
\frac{\partial a}{\partial \bar{z}}=0 \quad \text { in } \Omega, \quad \frac{\partial d}{\partial z}=0 \quad \text { in } \Omega,\left.\quad\left(a e^{\mathcal{A}}+d e^{\mathcal{B}}\right)\right|_{\partial \Omega}=\gamma
$$

Consider the range of the operator $R$. Clearly it is closed, because it lies in a finite-dimensional space. Let us show that the point $(0, \ldots, 0,1,1)$ belongs to the image of $R$. Let a holomorphic function $a$ satisfy

$$
\frac{\partial^{\beta} a}{\partial x_{1}^{\beta_{1}} \partial x_{2}^{\beta_{2}}}\left(y_{j}\right)=0 \quad \forall|\beta| \in\{0, \ldots, 5\}, j \in\{1, \ldots, \widetilde{k}\}, \quad|a(\widetilde{x})|>2 .
$$

Consider the function $-e^{\mathcal{A}-\mathcal{B}} a(z)$ and the pair $\left(b_{1}, b_{2}\right)=\left(\operatorname{Re}\left\{-e^{\mathcal{A}-\mathcal{B}} a\right\}\right.$, $\left.\operatorname{Im}\left\{e^{\mathcal{A}-\mathcal{B}} a\right\}\right)$. Using Proposition 7.1 we solve problem (7.1) with $l=0$ approximately. Let $\left(\phi_{\epsilon}, \psi_{\epsilon}\right)$ be a sequence of functions such that

$\frac{\partial}{\partial \bar{z}}\left(\phi_{\epsilon}+i \psi_{\epsilon}\right)=0 \quad$ in $\Omega,\left.\quad\left(\phi_{\epsilon}, \psi_{\epsilon}\right)\right|_{\Gamma_{0}} \rightarrow\left(b_{1}, b_{2}\right) \quad$ in $C^{5+\alpha}\left(\overline{\Gamma_{0}}\right), \quad\left(\phi_{\epsilon}+i \psi_{\epsilon}\right)(\widetilde{x}) \rightarrow 1$.

Denote $d_{\epsilon}=\phi_{\epsilon}-i \psi_{\epsilon}$ and $\beta_{\epsilon}=a e^{\mathcal{A}}+d_{\epsilon} e^{\mathcal{B}}$. Then $\left\{\beta_{\epsilon}\right\}$ converges to zero in $C^{5+\alpha}\left(\Gamma_{0}\right)$.

By Proposition 2.3 there exists a solution to problem (2.13) with initial data $\beta_{\epsilon}$, which we denote by $\left\{\widetilde{a}_{\epsilon}, \widetilde{d}_{\epsilon}\right\}$, such that the sequence $\left\{\widetilde{a}_{\epsilon}, \widetilde{d}_{\epsilon}\right\}$ converges to zero in $\left(C^{5}(\bar{\Omega})\right)^{2}$. Denote $\gamma_{\epsilon}=\left.\left(a+\widetilde{a}_{\epsilon}, d_{\epsilon}+\widetilde{d}_{\epsilon}\right)\right|_{\Gamma_{0}}$. Clearly $R\left(\gamma_{\epsilon}\right)$ converges to $(0, \ldots, 0,1,1)$. 


\section{§8. Appendix II. Asymptotic formulae}

Recall that we identify $x=\left(x_{1}, x_{2}\right) \in \mathbb{R}^{2}$ with $z=x_{1}+i x_{2} \in \mathbb{C}$, and that $\widetilde{g}_{1}, \widetilde{g}_{2}, \widetilde{g}_{3}, \widetilde{g}_{4}$ are defined just after (4.13) and (4.42).

Proposition 8.1. Under the conditions of Theorem 1.3, for any point $x$ on the boundary of $\Omega$, we have, as $|\tau| \rightarrow+\infty$,

$$
\begin{aligned}
& -\frac{1}{\pi} \int_{\Omega} \frac{e_{1} \widetilde{g}_{1} e^{-\tau(\Phi(\zeta)-\overline{\Phi(\zeta)})}}{\zeta-z} d \xi_{1} d \xi_{2} \\
& =\frac{\Lambda_{-}(\tau, \widetilde{x})}{\tau^{2}}\left(\frac{\frac{\partial_{z} \widetilde{z}_{1}(\widetilde{x})}{\partial_{z}^{2} \Phi(\widetilde{x})}}{(\widetilde{z}-z)^{2}}+\frac{\frac{\partial_{z} \widetilde{g}_{1}(\widetilde{x})}{\partial_{z}^{2} \Phi(\widetilde{x})} \frac{\partial_{z}^{3} \Phi(\widetilde{x})}{\partial_{z}^{2} \Phi(\widetilde{x})}+\frac{\partial_{z}^{2} \widetilde{g}_{1}(\widetilde{x})}{\partial_{z}^{2} \Phi(\widetilde{x})}-\frac{\partial_{z}^{2} \widetilde{g}_{1}(\widetilde{x})}{\partial_{z}^{2} \Phi(\widetilde{x})}}{2(\widetilde{z}-z)}\right)+o\left(1 / \tau^{2}\right), \\
& -\frac{1}{\pi} \int_{\Omega} \frac{e_{1} \widetilde{g}_{2} e^{\tau(\Phi(\zeta)-\overline{\Phi(\zeta))}}}{\bar{\zeta}-\bar{z}} d \xi_{1} d \xi_{2} \\
& =\frac{\Lambda_{+}(\tau, \widetilde{x})}{\tau^{2}}\left(\frac{\frac{\partial_{\bar{z}} \widetilde{g}_{2}(\widetilde{x})}{\bar{\partial}_{z}^{2} \Phi(\widetilde{x})}}{(\widetilde{\widetilde{z}}-\bar{z})^{2}}+\frac{\frac{\partial_{z} \widetilde{g}_{2}(\widetilde{x})}{\overline{\partial_{z}^{2} \Phi(\widetilde{x})}} \frac{\partial_{z}^{3} \bar{\Phi}(\widetilde{x})}{\partial_{\tilde{z}}^{2} \bar{\Phi}(\widetilde{x})}-\frac{\partial_{\bar{z}}^{2} \widetilde{g}_{2}(\widetilde{x})}{\partial_{z}^{2} \Phi(\widetilde{x})}+\frac{\partial_{z}^{2} \widetilde{g}_{2}(\widetilde{x})}{\partial_{z}^{2} \Phi(\widetilde{x})}}{2(\overline{\widetilde{z}}-\bar{z})}\right)+o\left(1 / \tau^{2}\right), \\
& -\frac{1}{\pi} \int_{\Omega} \frac{e_{1} \widetilde{g}_{3} e^{-\tau(\Phi(\zeta)-\overline{\Phi(\zeta)})}}{\bar{\zeta}-\bar{z}} d \xi_{1} d \xi_{2} \\
& =\frac{\Lambda_{-}(\tau, \widetilde{x})}{\tau^{2}}\left(\frac{\frac{\partial_{z} \widetilde{z}_{3}(\widetilde{x})}{\partial_{z}^{2} \Phi(\widetilde{x})}}{(\overline{\widetilde{z}}-\bar{z})^{2}}+\frac{\frac{\partial_{\bar{z}} \widetilde{g}_{3}(\widetilde{x})}{\partial_{\bar{z}}^{2} \bar{\Phi}(\widetilde{x})} \frac{\partial_{\bar{z}}^{3} \bar{\Phi}(\widetilde{x})}{\partial \frac{2}{\bar{z}} \bar{\Phi}(\widetilde{x})}-\frac{\partial_{\bar{z}}^{2} \widetilde{g}_{3}(\widetilde{x})}{\bar{z}_{z}^{2} \Phi(\widetilde{x})}+\frac{\partial_{z}^{2} \widetilde{g}_{3}(\widetilde{x})}{\partial_{z}^{2} \Phi(\widetilde{x})}}{2(\overline{\widetilde{z}}-\bar{z})}\right)+o\left(1 / \tau^{2}\right), \\
& -\frac{1}{\pi} \int_{\Omega} \frac{e_{1} \widetilde{g}_{4} e^{\tau(\Phi(\zeta)-\overline{\Phi(\zeta))}}}{\zeta-z} d \xi_{1} d \xi_{2} \\
& =\frac{\Lambda_{+}(\tau, \widetilde{x})}{\tau^{2}}\left(\frac{\frac{\partial_{z} \widetilde{g}_{4}(\widetilde{x})}{\partial_{z}^{2} \Phi(\widetilde{x})}}{(\widetilde{z}-z)^{2}}+\frac{\frac{\partial_{z} \widetilde{g}_{4}(\widetilde{x})}{\partial_{z}^{2} \Phi(\widetilde{x})} \frac{\partial_{z}^{3} \Phi(\widetilde{x})}{\partial_{z}^{2} \Phi(\widetilde{x})}+\frac{\partial_{z}^{2} \widetilde{g}_{4}(\widetilde{x})}{\partial_{z}^{2} \Phi(\widetilde{x})}-\frac{\partial_{z}^{2} \widetilde{g}_{4}(\widetilde{x})}{\partial_{z}^{2} \Phi(\widetilde{x})}}{2(\widetilde{z}-z)}\right)+o\left(1 / \tau^{2}\right) .
\end{aligned}
$$

Proof. Let $\delta>0$ be sufficiently small and $\widetilde{e} \in C_{0}^{\infty}(B(\widetilde{x}, \delta))$ with $\left.\widetilde{e}\right|_{B(\widetilde{x}, \delta / 2)} \equiv 1$. Let $\widetilde{g} \in C^{2}(\bar{\Omega})$ be some function such that $\widetilde{g}(\widetilde{x})=\frac{\partial \widetilde{g}}{\partial \bar{z}}(\widetilde{x})=0$. We compute the asymptotics of the following integral as $|\tau|$ goes to infinity:

$$
\quad-\frac{1}{\pi} \int_{\Omega} \frac{e_{1} \widetilde{g} e^{-\tau(\Phi(\zeta)-\overline{\Phi(\zeta)})}}{\zeta-z} d \xi_{1} d \xi_{2}
$$

$$
\begin{aligned}
& =-\frac{1}{\pi} \int_{B(\widetilde{x}, \delta)} \frac{\tilde{e} \widetilde{g} e^{-\tau(\Phi(\zeta)-\overline{\Phi(\zeta))}}}{\zeta-z} d \xi_{1} d \xi_{2}+o\left(1 / \tau^{2}\right) \\
& =-\frac{1}{\pi} \int_{B(\widetilde{x}, \delta)} \widetilde{e}\left\{\frac{\partial_{z} \widetilde{g}(\widetilde{x})(\zeta-\widetilde{z})+\frac{1}{2} \partial_{\bar{z}}^{2} \widetilde{g}(\widetilde{x})(\bar{\zeta}-\overline{\widetilde{z}})^{2}}{\zeta-z}\right.
\end{aligned}
$$

$$
\left.+\frac{\partial_{\bar{z}} \partial_{z} \widetilde{g}(\widetilde{x})(\zeta-\widetilde{z})(\bar{\zeta}-\overline{\widetilde{z}})+\frac{1}{2} \partial_{z}^{2} \widetilde{g}(\widetilde{x})(\zeta-\widetilde{z})^{2}}{\zeta-z}\right\} e^{-\tau(\Phi(\zeta)-\overline{\Phi(\zeta)})} d \xi_{1} d \xi_{2}+o\left(1 / \tau^{2}\right)
$$




$$
\begin{aligned}
& =-\frac{1}{\pi} \int_{B(\widetilde{x}, \delta)} \widetilde{e}\left\{\frac{\frac{\partial_{z} \widetilde{g}(\widetilde{x})}{\partial_{z}^{2} \Phi(\widetilde{x})}\left(\partial_{\zeta} \Phi-\frac{1}{2} \partial_{z}^{3} \Phi(\widetilde{x})(\zeta-\widetilde{z})^{2}\right)+\frac{1}{2} \frac{\partial_{\bar{z}}^{2} \widetilde{g}(\widetilde{x})}{\partial_{z}^{2} \Phi(\widetilde{x})} \partial_{\bar{\zeta}} \bar{\Phi}(\bar{\zeta}-\overline{\widetilde{z}})}{\zeta-z}\right. \\
& \left.+\frac{\frac{\partial_{\bar{z}} \partial_{z} \widetilde{g}(\widetilde{x})}{\partial_{z}^{2} \Phi(\widetilde{x})}(\zeta-\widetilde{z}) \partial_{\bar{\zeta}} \bar{\Phi}+\frac{1}{2} \frac{\partial_{z}^{2} \widetilde{g}(\widetilde{x})}{\partial_{z}^{2} \Phi(\widetilde{x})} \partial_{\zeta} \Phi(\zeta-\widetilde{z})}{\zeta-z}\right\} e^{-\tau(\Phi(\zeta)-\overline{\Phi(\zeta)})} d \xi_{1} d \xi_{2}+o\left(1 / \tau^{2}\right) \\
& =-\frac{1}{\pi} \int_{B(\widetilde{x}, \delta)} \widetilde{e}\left\{\frac{\frac{\partial_{z} \widetilde{g}(\widetilde{x})}{\partial_{z}^{2} \Phi(\widetilde{x})}\left(\partial_{\zeta} \Phi-\frac{1}{2} \frac{\partial_{z}^{3} \Phi(\widetilde{x})}{\partial_{z}^{2} \Phi(\widetilde{x})} \partial_{\zeta} \Phi(\zeta-\widetilde{z})\right)+\frac{1}{2} \frac{\partial_{\bar{z}}^{2} \widetilde{g}(\widetilde{x})}{\partial_{z}^{2} \Phi(\widetilde{x})} \partial_{\bar{\zeta}} \bar{\Phi}(\bar{\zeta}-\overline{\widetilde{z}})}{\zeta-z}\right. \\
& \left.+\frac{\frac{\partial_{z} \partial_{z} \widetilde{g}(\widetilde{x})}{\partial_{z}^{2} \Phi(\widetilde{x})}(\zeta-\widetilde{z}) \partial_{\bar{\zeta}} \bar{\Phi}+\frac{1}{2} \frac{\partial_{z}^{2} \widetilde{g}(\widetilde{x})}{\partial_{z}^{2} \Phi(\widetilde{x})} \partial_{\zeta} \Phi(\zeta-\widetilde{z})}{\zeta-z}\right\} e^{-\tau(\Phi(\zeta)-\overline{\Phi(\zeta)})} d \xi_{1} d \xi_{2}+o\left(1 / \tau^{2}\right) \\
& =-\frac{1}{\pi \tau} \int_{B(\widetilde{x}, \delta)} \widetilde{e}\left\{\frac{-\frac{\partial_{z} \widetilde{g}(\widetilde{x})}{\partial_{z}^{2} \Phi(\widetilde{x})} \frac{\partial_{z}^{3} \Phi(\widetilde{x})}{\partial_{z}^{2} \Phi(\widetilde{x})}-\frac{\partial_{z}^{2} \widetilde{g}(\widetilde{x})}{\partial_{z}^{2} \Phi(\widetilde{x})}}{2(\zeta-z)}-\frac{\frac{\partial_{z} \widetilde{g}(\widetilde{x})}{\partial_{z}^{2} \Phi(\widetilde{x})}\left(1-\frac{1}{2} \frac{\partial_{z}^{3} \Phi(\widetilde{x})}{\partial_{z}^{2} \Phi(\widetilde{x})}(\zeta-\widetilde{z})\right)}{(\zeta-z)^{2}}\right. \\
& \left.+\frac{\frac{\partial_{z}^{2} \widetilde{g}(\widetilde{x})}{\partial_{z}^{2} \Phi(\widetilde{x})}}{2(\zeta-z)}-\frac{\frac{\partial_{z}^{2} \widetilde{g}(\widetilde{x})}{\partial_{z}^{2} \Phi(\widetilde{x})}(\zeta-\widetilde{z})}{2(\zeta-z)^{2}}\right\} e^{-\tau(\Phi(\zeta)-\overline{\Phi(\zeta)})} d \xi_{1} d \xi_{2}+o\left(1 / \tau^{2}\right) .
\end{aligned}
$$

Here we used

$$
\int_{B(\widetilde{x}, \delta)} \widetilde{e} \frac{\zeta-\widetilde{z}}{(\zeta-z)^{2}} e^{-\tau(\Phi(\zeta)-\overline{\Phi(\zeta)})} d \xi_{1} d \xi_{2}=o(1 / \tau) \quad \text { as }|\tau| \rightarrow+\infty,
$$

which is obtained by stationary phase. Another asymptotic calculation is

$$
\begin{array}{r}
-\frac{1}{\pi} \int_{\Omega} \frac{e_{1} \widetilde{g} e^{\tau(\Phi(\zeta)-\overline{\Phi(\zeta)})}}{\zeta-z} d \xi_{1} d \xi_{2}=\frac{1}{\pi \tau} \int_{B(\widetilde{x}, \delta)} \widetilde{e} \frac{\frac{\partial_{z} \widetilde{g}(\widetilde{x})}{\partial_{z}^{2} \Phi(\widetilde{x})}}{(\zeta-z)^{2}} e^{-\tau(\Phi(\zeta)-\overline{\Phi(\zeta)})} d x \\
+\frac{1}{2 \pi \tau} \int_{B(\widetilde{x}, \delta)} \widetilde{e} \frac{\frac{\partial}{z} \widetilde{g}(\widetilde{x})_{\partial_{z}^{2} \Phi(\widetilde{x})} \frac{\partial_{z}^{3} \Phi(\widetilde{x})}{\partial_{z}^{2} \Phi(\widetilde{x})}+\frac{\partial_{z}^{2} \widetilde{g}(\widetilde{x})}{\partial_{z}^{2} \Phi(\widetilde{x})}-\frac{\partial_{z}^{2} \widetilde{g}(\widetilde{x})}{\partial_{z}^{2} \Phi(\widetilde{x})}}{\zeta-z} e^{-\tau(\Phi(\zeta)-\overline{\Phi(\zeta)})} d x+o\left(1 / \tau^{2}\right) \\
=\frac{\Lambda_{-}(\tau, \widetilde{x})}{\tau^{2}}\left(\frac{\frac{\partial_{z} \widetilde{g}(\widetilde{x})}{\partial_{z}^{2} \Phi(\widetilde{x})}}{(\widetilde{z}-z)^{2}}+\frac{\frac{\partial_{z} \widetilde{g}(\widetilde{x})}{\partial_{z}^{2} \Phi(\widetilde{x})} \frac{\partial_{z}^{3} \Phi(\widetilde{x})}{\partial_{z}^{2} \Phi(\widetilde{x})}+\frac{\partial_{z}^{2} \widetilde{g}(\widetilde{x})}{\partial_{z}^{2} \Phi(\widetilde{x})}-\frac{\partial_{z}^{2} \widetilde{g}(\widetilde{x})}{\partial_{z}^{2} \Phi(\widetilde{x})}}{2(\widetilde{z}-z)}\right) \\
+o\left(1 / \tau^{2}\right) \quad \text { as }|\tau| \rightarrow+\infty .
\end{array}
$$

Taking $\widetilde{g}=g_{1}$ and $\widetilde{g}=\bar{g}_{3}$, we obtain (8.1) and (8.3) from the above formula. Taking $\widetilde{g}=g_{4}$ and $\widetilde{g}=\bar{g}_{2}$ and replacing $\tau$ by $-\tau$, we obtain (8.4) and (8.2).

Proposition 8.2. For any $x$ from the boundary of $\Omega$, the following asymptotic formulae hold true as $|\tau| \rightarrow+\infty$ :

$$
\mathfrak{G}_{1}(x, \tau)=-\frac{\Lambda_{-}(\tau, \widetilde{x})}{2 \tau}\left(\frac{\frac{\partial\left(g_{1} e^{-\mathcal{A}_{1}}\right)}{\partial z}(\widetilde{x})+\frac{\partial \Phi}{\partial z} m_{1}(\widetilde{x})}{\widetilde{z}-z}+\frac{\sigma_{1}(\widetilde{x}) \frac{\partial \Phi}{\partial z}}{(\widetilde{z}-z)^{2}}\right)+o(1 / \tau),
$$




$$
\begin{aligned}
& \mathfrak{G}_{2}(x, \tau)=-\frac{\Lambda_{+}(\tau, \widetilde{x})}{2 \tau}\left(\frac{\frac{\partial\left(g_{2} e^{-\mathcal{B}_{1}}\right)}{\partial \bar{z}}(\widetilde{x})+\frac{\partial \Phi}{\partial \bar{z}} \widetilde{m}_{1}(\widetilde{x})}{\overline{\widetilde{z}}-\bar{z}}+\frac{\widetilde{\sigma}_{1}(\widetilde{x}) \frac{\partial \Phi}{\partial \bar{z}}}{(\overline{\widetilde{z}}-\bar{z})^{2}}\right)+o(1 / \tau), \\
& \mathfrak{G}_{3}(x, \tau)=-\frac{\Lambda_{-}(\tau, \widetilde{x})}{2 \tau}\left(\frac{\frac{\partial\left(g_{3} e^{\left.-\mathcal{A}_{2}\right)}\right.}{\partial \bar{z}}(\widetilde{x})-\frac{\partial \Phi}{\partial \bar{z}} t_{1}(\widetilde{x})}{\overline{\widetilde{z}}-\bar{z}}-\frac{r_{1}(\widetilde{x}) \frac{\partial \Phi}{\partial \bar{z}}}{(\overline{\widetilde{z}}-\bar{z})^{2}}\right)+o(1 / \tau), \\
& \mathfrak{G}_{4}(x, \tau)=-\frac{\Lambda_{+}(\tau, \widetilde{x})}{2 \tau}\left(\frac{\frac{\partial\left(g_{4} e^{-\mathcal{B}_{2}}\right)}{\partial z}(\widetilde{x})-\frac{\partial \Phi}{\partial z} \widetilde{t}_{1}(\widetilde{x})}{\widetilde{z}-z}-\frac{\widetilde{r}_{1}(\widetilde{x}) \frac{\partial \Phi}{\partial z}}{(\widetilde{z}-z)^{2}}\right)+o(1 / \tau) .
\end{aligned}
$$

Here $\widetilde{z}=\widetilde{x}_{1}+i \widetilde{x}_{2}$ and $m_{1}, \widetilde{m}_{1}, \sigma_{1}, \widetilde{\sigma}_{1}, t_{1}, \widetilde{t}_{1}, r_{1}, \widetilde{r}_{1}$ are introduced in (4.12), (4.13), (4.41) and (4.42). Moreover for sufficiently small positive $\epsilon$,

$$
\begin{aligned}
\left\|\frac{\partial \mathfrak{G}_{1}(\cdot, \tau)}{\partial \bar{z}}\right\|_{C\left(\overline{\mathcal{O}_{\epsilon}}\right)}+ & \left\|\frac{\partial \mathfrak{G}_{2}(\cdot, \tau)}{\partial z}\right\|_{C\left(\overline{\mathcal{O}_{\epsilon}}\right)}+\left\|\frac{\partial \mathfrak{G}_{3}(\cdot, \tau)}{\partial z}\right\|_{C\left(\overline{\mathcal{O}_{\epsilon}}\right)} \\
& +\left\|\frac{\partial \mathfrak{G}_{4}(\cdot, \tau)}{\partial \bar{z}}\right\|_{C\left(\overline{\mathcal{O}_{\epsilon}}\right)}=o(1 / \tau) \quad \text { as }|\tau| \rightarrow+\infty
\end{aligned}
$$

Proof. Observe that the functions $\mathfrak{G}_{k}(x, \tau), k=1,2,3,4$, are given by

$$
\begin{aligned}
& \mathfrak{G}_{1}(x, \tau)=-\frac{1}{2 \pi} \int_{\Omega} \frac{\frac{\partial \mathcal{A}_{1}(\zeta, \bar{\zeta})}{\partial \zeta}+\tau \frac{\partial \Phi}{\partial \zeta}(\zeta)-\left(\frac{\partial \mathcal{A}_{1}(z, \bar{z})}{\partial z}+\tau \frac{\partial \Phi}{\partial z}(z)\right)}{\zeta-z} \\
& \times\left(e_{1} g_{1} e^{-\mathcal{A}_{1}}\right)\left(\xi_{1}, \xi_{2}\right) e^{\tau(\overline{\Phi(\zeta)}-\Phi(\zeta))} d \xi_{1} d \xi_{2}, \\
& \mathfrak{G}_{2}(x, \tau)=-\frac{1}{2 \pi} \int_{\Omega} \frac{\frac{\partial \mathcal{B}_{1}(\zeta, \bar{\zeta})}{\partial \bar{\zeta}}+\tau \frac{\overline{\partial \Phi}(\zeta)}{\partial \zeta}-\left(\frac{\partial \mathcal{B}_{1}(z, \bar{z})}{\partial \bar{z}}+\tau \frac{\partial \Phi}{\partial \bar{z}}(\bar{z})\right)}{\bar{\zeta}-\bar{z}} \\
& \times\left(e_{1} g_{2} e^{-\mathcal{B}_{1}}\right)\left(\xi_{1}, \xi_{2}\right) e^{\tau(\Phi(\zeta)-\overline{\Phi(\zeta)})} d \xi_{1} d \xi_{2}, \\
& \mathfrak{G}_{3}(x, \tau)=\frac{1}{2 \pi} \int_{\Omega} \frac{\tau \frac{\partial \bar{\Phi}(\bar{\zeta})}{\partial \bar{\zeta}}-\frac{\partial \mathcal{A}_{2}(\bar{\zeta}, \bar{\zeta})}{\partial \bar{\zeta}}-\left(\tau \frac{\partial \bar{\Phi}(\bar{z})}{\partial \bar{z}}-\frac{\partial \mathcal{A}_{2}(z, \bar{z})}{\partial \bar{z}}\right)}{\bar{\zeta}-\bar{z}} \\
& \times\left(e_{1} g_{3} e^{-\mathcal{A}_{2}}\right)\left(\xi_{1}, \xi_{2}\right) e^{\tau(\overline{\Phi(\zeta)}-\Phi(\zeta))} d \xi_{1} d \xi_{2}, \\
& \mathfrak{G}_{4}(x, \tau)=\frac{1}{2 \pi} \int_{\Omega} \frac{\tau \frac{\partial \Phi(\zeta)}{\partial \zeta}-\frac{\partial \mathcal{B}_{2}(\zeta, \bar{\zeta})}{\partial \zeta}-\left(\tau \frac{\partial \Phi(z)}{\partial z}-\frac{\partial \mathcal{B}_{2}(z, \bar{z})}{\partial z}\right)}{\zeta-z} \\
& \times\left(e_{1} g_{4} e^{-\mathcal{B}_{2}}\right)\left(\xi_{1}, \xi_{2}\right) e^{\tau(\Phi(\zeta)-\overline{\Phi(\zeta)})} d \xi_{1} d \xi_{2} .
\end{aligned}
$$

Let $z=x_{1}+i x_{2}$ where $x=\left(x_{1}, x_{2}\right) \in \partial \Omega$. By Proposition 3.5,

$$
\begin{aligned}
\frac{\tau}{2 \pi} \int_{\Omega} \frac{\partial_{\zeta} \Phi(\zeta)}{\zeta-z}\left(e_{1} g_{1} e^{-\mathcal{A}_{1}}\right) e^{\tau(\overline{\Phi(\zeta)}-\Phi(\zeta))} d \xi_{1} d \xi_{2} \\
=-\frac{1}{2 \pi} \int_{\Omega} \frac{e_{1} g_{1} e^{-\mathcal{A}_{1}}}{\zeta-z} \frac{\partial}{\partial \zeta} e^{\tau(\overline{\Phi(\zeta)}-\Phi(\zeta))} d \xi_{1} d \xi_{2}
\end{aligned}
$$




$$
\begin{aligned}
& =\frac{1}{2 \pi} \int_{\Omega} \frac{\partial}{\partial \zeta}\left(\frac{e_{1} g_{1} e^{-\mathcal{A}_{1}}}{\zeta-z}\right) e^{\tau(\overline{\Phi(\zeta)}-\Phi(\zeta))} d \xi_{1} d \xi_{2} \\
& =\frac{\Lambda_{-}(\tau, \widetilde{x})}{2 \tau} \frac{\partial_{z}\left(g_{1} e^{-\mathcal{A}_{1}}\right)(\widetilde{x})}{\widetilde{z}-z}+o(1 / \tau), \\
& \frac{\tau}{2 \pi} \int_{\Omega} \frac{\partial_{\bar{\zeta}} \bar{\Phi}(\bar{\zeta})}{\bar{\zeta}-\bar{z}}\left(e_{1} g_{2} e^{-\mathcal{B}_{1}}\right) e^{\tau(\Phi(\zeta)-\overline{\Phi(\zeta)})} d \xi_{1} d \xi_{2} \\
& =-\frac{1}{2 \pi} \int_{\Omega} \frac{e_{1} g_{2} e^{-\mathcal{B}_{1}}}{\bar{\zeta}-\bar{z}} \frac{\partial}{\partial \bar{\zeta}} e^{\tau(\Phi(\zeta)-\overline{\Phi(\zeta)})} d \xi_{1} d \xi_{2} \\
& =\frac{1}{2 \pi} \int_{\Omega} \frac{\partial}{\partial \bar{\zeta}}\left(\frac{e_{1} g_{2} e^{-\mathcal{B}_{1}}}{\bar{\zeta}-\bar{z}}\right) e^{\tau(\Phi(\zeta)-\overline{\Phi(\zeta)})} d \xi_{1} d \xi_{2} \\
& =\frac{\Lambda_{+}(\tau, \widetilde{x})}{2 \tau} \frac{\partial_{\bar{z}}\left(g_{2} e^{-\mathcal{B}_{1}}\right)(\widetilde{x})}{\overline{\widetilde{z}}-\bar{z}}+o(1 / \tau), \\
& \frac{\tau}{2 \pi} \int_{\Omega} \frac{\partial_{\bar{\zeta}} \bar{\Phi}}{\bar{\zeta}-\bar{z}}\left(e_{1} g_{3} e^{-\mathcal{A}_{2}}\right) e^{\tau(\overline{\Phi(\zeta)}-\Phi(\zeta))} d \xi_{1} d \xi_{2} \\
& =\frac{1}{2 \pi} \int_{\Omega} \frac{e_{1} g_{3} e^{-\mathcal{A}_{2}}}{\bar{\zeta}-\bar{z}} \frac{\partial}{\partial \bar{\zeta}} e^{\tau(\overline{\Phi(\zeta)}-\Phi(\zeta))} d \xi_{1} d \xi_{2} \\
& =-\frac{1}{2 \pi} \int_{\Omega} \frac{\partial}{\partial \bar{\zeta}}\left(\frac{e_{1} g_{3} e^{-\mathcal{A}_{2}}}{\bar{\zeta}-\bar{z}}\right) e^{\tau(\overline{\Phi(\zeta)}-\Phi(\zeta))} d \xi_{1} d \xi_{2} \\
& =-\frac{\Lambda_{-}(\tau, \widetilde{x})}{2 \tau} \frac{\partial_{\bar{z}}\left(g_{3} e^{-\mathcal{A}_{2}}\right)(\widetilde{x})}{\overline{\widetilde{z}}-\bar{z}}+o(1 / \tau), \\
& \frac{\tau}{2 \pi} \int_{\Omega} \frac{\partial_{\zeta} \Phi(\zeta)}{\zeta-z}\left(e_{1} g_{4} e^{-\mathcal{B}_{2}}\right) e^{\tau(\Phi(\zeta)-\overline{\Phi(\zeta)})} d \xi_{1} d \xi_{2} \\
& =\frac{1}{2 \pi} \int_{\Omega} \frac{e_{1} g_{4} e^{-\mathcal{B}_{2}}}{\zeta-z} \frac{\partial}{\partial \zeta} e^{\tau(\Phi(\zeta)-\overline{\Phi(\zeta)})} d \xi_{1} d \xi_{2} \\
& =-\frac{1}{2 \pi} \int_{\Omega} \frac{\partial}{\partial \zeta}\left(\frac{e_{1} g_{4} e^{-\mathcal{B}_{2}}}{\zeta-z}\right) e^{\tau(\Phi(\zeta)-\overline{\Phi(\zeta)})} d \xi_{1} d \xi_{2} \\
& =-\frac{\Lambda_{+}(\tau, \widetilde{x})}{2 \tau} \frac{\partial_{z}\left(g_{4} e^{-\mathcal{B}_{2}}\right)(\widetilde{x})}{\widetilde{z}-z}+o(1 / \tau) .
\end{aligned}
$$

Noting that $\widetilde{g}_{1}=e^{-\mathcal{A}_{1}} g_{1}, \widetilde{g}_{2}=e^{-\mathcal{B}_{1}} g_{2}, \widetilde{g}_{3}=e^{-\mathcal{A}_{2}} g_{3}$ and $\widetilde{g}_{4}=e^{-\mathcal{B}_{2}} g_{4}$, and taking into account Proposition 8.1, we obtain (8.7)-(8.10) for the functions $\mathfrak{G}_{k}(x, \tau)$, $k=1,2,3,4$.

To prove the estimate (8.11), it suffices to show that

$$
\left\|\frac{\partial \mathfrak{G}_{1}(\cdot, \tau)}{\partial \bar{z}}\right\|_{C\left(\overline{\mathcal{O}_{\epsilon}}\right)}=o(1 / \tau)
$$

In fact,

$$
\partial_{\bar{z}} \mathfrak{G}_{1}(x, \tau)=-\frac{1}{4 \pi} \frac{\partial A_{1}}{\partial z} \int_{\Omega} \frac{\left(e_{1} g_{1} e^{-\mathcal{A}_{1}}\right)\left(\xi_{1}, \xi_{2}\right) e^{\tau(\overline{\Phi(\zeta)}-\Phi(\zeta))}}{\zeta-z} d \xi_{1} d \xi_{2}
$$

Then Proposition 3.5 and (4.6) yield the desired estimate. 
Proof of Proposition 5.2. Using (5.13) and (5.16), we have

$$
\mathfrak{L}_{0} \equiv\left(2\left(A_{1}-A_{2}\right) \frac{\partial U_{1}}{\partial z}, b_{\tau} e^{\mathcal{B}_{2}-\tau \Phi}+c_{\tau} e^{\mathcal{A}_{2}-\tau \bar{\Phi}}\right)_{L^{2}(\Omega)}
$$

$$
+\left(2\left(B_{1}-B_{2}\right) \frac{\partial U_{1}}{\partial \bar{z}}, b_{\tau} e^{\mathcal{B}_{2}-\tau \Phi}+c_{\tau} e^{\mathcal{A}_{2}-\tau \bar{\Phi}}\right)_{L^{2}(\Omega)}
$$

$=2\left(\left(A_{1}-A_{2}\right) e^{\tau \Phi}\left(-\mathcal{R}_{-\tau, A_{1}}\left\{\frac{\partial\left(e_{1} g_{1}\right)}{\partial z}\right\}+e^{\mathcal{A}_{1}-\tau(\bar{\Phi}-\Phi)} \mathfrak{G}_{1}(\cdot, \tau)\right), c_{\tau} e^{\mathcal{A}_{2}-\tau \bar{\Phi}}\right)_{L^{2}(\Omega)}$

$-2\left(\left(A_{1}-A_{2}\right) e^{\tau \bar{\Phi}} \frac{\partial}{\partial z} \mathcal{R}_{-\tau, A_{1}}\left\{e_{1} g_{1}\right\}, b_{\tau} e^{\mathcal{B}_{2}-\tau \Phi}\right)_{L^{2}(\Omega)}$

$+\left(\left(B_{1}-B_{2}\right)\left(-e_{1} g_{1}+A_{1} \mathcal{R}_{-\tau, A_{1}}\left\{e_{1} g_{1}\right\}\right) e^{\tau \bar{\Phi}}, b_{\tau} e^{\mathcal{B}_{2}-\tau \Phi}+c_{\tau} e^{\mathcal{A}_{2}-\tau \bar{\Phi}}\right)_{L^{2}(\Omega)}$

$+o(1 / \tau)$.

By (4.10) and Proposition 3.4 we have

$$
\begin{aligned}
& 2\left(\left(A_{1}-A_{2}\right) e^{\tau \bar{\Phi}} \frac{\partial}{\partial z} \mathcal{R}_{-\tau, A_{1}}\left\{e_{1} g_{1}\right\}, b_{\tau} e^{\mathcal{B}_{2}-\tau \Phi}\right)_{L^{2}(\Omega)} \\
&=2\left(\left(A_{1}-A_{2}\right) \frac{\partial}{\partial z} \mathcal{R}_{-\tau, A_{1}}\left\{e_{1} g_{1}\right\}, b_{\tau} e^{\mathcal{B}_{2}}\right)_{L^{2}(\Omega)} \\
&=\left(\left(A_{1}-A_{2}\right)\left(\nu_{1}-i \nu_{2}\right) \mathcal{R}_{-\tau, A_{1}}\left\{e_{1} g_{1}\right\}, b_{\tau} e^{\mathcal{B}_{2}}\right)_{L^{2}(\partial \Omega)} \\
&-2\left(\mathcal{R}_{-\tau, A_{1}}\left\{e_{1} g_{1}\right\}, \frac{\partial}{\partial \bar{z}}\left\{b_{\tau} e^{\mathcal{B}_{2}} \overline{\left(A_{1}-A_{2}\right)}\right\}\right)_{L^{2}(\Omega)}+o(1 / \tau) \quad \text { as }|\tau| \rightarrow+\infty \\
&=-\left(\frac{e_{1} g_{1}}{\tau \overline{\partial_{z} \Phi}}, \frac{\partial}{\partial \bar{z}}\left\{b_{\tau} e^{\mathcal{B}_{2}} \overline{\left(A_{1}-A_{2}\right)}\right\}\right)_{L^{2}(\Omega)}+o
\end{aligned}
$$

Using the stationary phase argument, we obtain

$$
\begin{aligned}
& -2\left(\left(A_{1}-A_{2}\right) e^{\tau \bar{\Phi}} \mathcal{R}_{-\tau, A_{1}}\left\{\frac{\partial\left(e_{1} g_{1}\right)}{\partial z}\right\}, c_{\tau} e^{\mathcal{A}_{2}-\tau \bar{\Phi}}\right)_{L^{2}(\Omega)} \\
& =-\left(\left(A_{1}-A_{2}\right) e^{\mathcal{A}_{1}+\overline{\mathcal{A}_{2}}} \partial_{\bar{z}}^{-1}\left\{\frac{\partial\left(e_{1} g_{1}\right)}{\partial z} e^{-\mathcal{A}_{1}-\tau(\Phi-\bar{\Phi})}\right\}, c_{\tau}\right)_{L^{2}(\Omega)} \\
& =\frac{1}{\pi} \int_{\Omega}\left(A_{1}-A_{2}\right) e^{\mathcal{A}_{1}+\overline{\mathcal{A}_{2}}} \overline{c_{\tau}}\left(\int_{\Omega} \frac{\frac{\partial\left(e_{1} g_{1}\right)}{\partial \zeta} e^{-\mathcal{A}_{1}-\tau(\Phi-\bar{\Phi})}}{\zeta-z} d \xi_{1} d \xi_{2}\right) d x_{1} d x_{2} \\
& =\frac{1}{\pi} \int_{\Omega} \frac{\partial\left(e_{1} g_{1}\right)}{\partial \zeta} e^{-\mathcal{A}_{1}-\tau(\Phi-\bar{\Phi})}\left(\int_{\Omega} \frac{\left(A_{1}-A_{2}\right) \overline{c_{\tau}} e^{\mathcal{A}_{1}+\overline{\mathcal{A}_{2}}}}{\zeta-z} d x_{1} d x_{2}\right) d \xi_{1} d \xi_{2} \\
& =\frac{\Lambda_{-}(\tau, \widetilde{x})}{\tau} \frac{\partial g_{1}}{\partial z}(\widetilde{x}) e^{-\mathcal{A}_{1}(\widetilde{x})}\left(\int_{\Omega} \frac{\left(A_{1}-A_{2}\right) \bar{c} e^{\mathcal{A}_{1}+\overline{\mathcal{A}_{2}}}}{\widetilde{z}-z} d x_{1} d x_{2}\right)+o(1 / \tau) .
\end{aligned}
$$


Integrating by parts and using (8.11), (3.2), $2 \frac{\partial \mathcal{A}_{1}}{\partial \bar{z}}=-A_{1}$ and $2 \frac{\partial \mathcal{A}_{2}}{\partial z}=\overline{A_{2}}$ from (4.30), we obtain

$$
\begin{aligned}
& \text { (8.15) } \quad 2\left(\left(A_{1}-A_{2}\right) e^{\tau \bar{\Phi}} e^{\mathcal{A}_{1}} e^{-\tau(\bar{\Phi}-\Phi)} \mathfrak{G}_{1}, c_{\tau} e^{\mathcal{A}_{2}-\tau \bar{\Phi}}\right)_{L^{2}(\Omega)} \\
& =2\left(\left(A_{1}-A_{2}\right) e^{\mathcal{A}_{1}} \mathfrak{G}_{1}, c_{\tau} e^{\mathcal{A}_{2}}\right)_{L^{2}(\Omega)}=\int_{\Omega} 2\left(A_{1}-A_{2}\right) e^{\mathcal{A}_{1}+\overline{\mathcal{A}_{2}}} \overline{c_{\tau}(\bar{z})} \mathfrak{G}_{1}(x, \tau) d x \\
& =-4 \int_{\Omega} \frac{\partial}{\partial \bar{z}}\left(e^{\mathcal{A}_{1}+\overline{\mathcal{A}_{2}}}\right) \overline{c_{\tau}(\bar{z})} \mathfrak{G}_{1}(x, \tau) d x \\
& =4 \int_{\Omega} e^{\mathcal{A}_{1}+\overline{\mathcal{A}_{2}}} \overline{c_{\tau}(\bar{z})} \frac{\partial}{\partial \bar{z}} \mathfrak{G}_{1}(x, \tau) d x \\
& \quad-2 \int_{\partial \Omega}\left(\nu_{1}+i \nu_{2}\right) e^{\mathcal{A}_{1}+\overline{\mathcal{A}_{2}}} \overline{c_{\tau}(\bar{z})} \mathfrak{G}_{1}(x, \tau) d \sigma+o(1 / \tau) \quad \text { as }|\tau| \rightarrow+\infty .
\end{aligned}
$$

Since

$$
\begin{aligned}
e^{\mathcal{A}_{1}} \frac{\partial}{\partial \bar{z}} \mathfrak{G}_{1}(x, \tau) & =-\frac{e^{\mathcal{A}_{1}}}{4 \pi} \frac{\partial A_{1}}{\partial z} \int_{\Omega} \frac{\left(e_{1} g_{1} e^{-\mathcal{A}_{1}}\right)\left(\xi_{1}, \xi_{2}\right) e^{\tau(\overline{\Phi(\zeta)}-\Phi(\zeta))}}{\zeta-z} d \xi_{1} d \xi_{2} \\
& =\frac{1}{2} \frac{\partial A_{1}}{\partial z} e^{\tau(\bar{\Phi}-\Phi)} \mathcal{R}_{-\tau, A_{1}}\left\{e_{1} g_{1}\right\}
\end{aligned}
$$

applying Propositions 3.4 and 3.3 we obtain

$$
\int_{\Omega} e^{\mathcal{A}_{1}+\overline{\mathcal{A}_{2}}} \overline{c_{\tau}(\bar{z})} \frac{\partial}{\partial \bar{z}} \mathfrak{G}_{1}(x, \tau) d x=o(1 / \tau) \quad \text { as }|\tau| \rightarrow+\infty .
$$

By (8.7), (8.14)-(8.16) and Propositions 3.4 and 3.5, we conclude

$$
\begin{aligned}
& \mathfrak{L}_{0}=\left(\left(B_{1}-B_{2}\right)\left(-e_{1} g_{1}+\frac{A_{1} e_{1} g_{1}}{2 \tau \overline{\partial_{z} \Phi}}\right), b_{\tau} e^{\mathcal{B}_{2}}\right)_{L^{2}(\Omega)} \\
& +\left(\frac{e_{1} g_{1}}{\tau \overline{\partial_{z} \Phi}}, \frac{\partial}{\partial \bar{z}}\left\{b e^{\mathcal{B}_{2}} \overline{\left(A_{1}-A_{2}\right)}\right\}\right)_{L^{2}(\Omega)} \\
& +\frac{\Lambda_{-}(\tau, \widetilde{x})}{\tau} \frac{\partial g_{1}}{\partial z}(\widetilde{x}) e^{-\mathcal{A}_{1}(\widetilde{x})}\left(\int_{\Omega} \frac{\left(A_{1}-A_{2}\right) \bar{c} e^{\mathcal{A}_{1}+\overline{\mathcal{A}_{2}}}}{\widetilde{z}-z} d x_{1} d x_{2}\right) \\
& -2 \int_{\partial \Omega}\left(\nu_{1}+i \nu_{2}\right) e^{\mathcal{A}_{1}+\overline{\mathcal{A}_{2}}} \overline{c_{\tau}(\bar{z})} \mathfrak{G}_{1}(x, \tau) d \sigma+o(1 / \tau) \quad \text { as }|\tau| \rightarrow+\infty .
\end{aligned}
$$

Using (5.14) and (5.18), we obtain after simple computations

$$
\begin{aligned}
\mathfrak{L}_{1} \equiv & \left(2\left(A_{1}-A_{2}\right) \frac{\partial U_{2}}{\partial z}, b_{\tau} e^{\mathcal{B}_{2}-\tau \Phi}+c_{\tau} e^{\mathcal{A}_{2}-\tau \bar{\Phi}}\right)_{L^{2}(\Omega)} \\
& +\left(2\left(B_{1}-B_{2}\right) \frac{\partial U_{2}}{\partial \bar{z}}, b_{\tau} e^{\mathcal{B}_{2}-\tau \Phi}+c_{\tau} e^{\mathcal{A}_{2}-\tau \bar{\Phi}}\right)_{L^{2}(\Omega)}
\end{aligned}
$$




$$
\begin{aligned}
= & \left(\left(A_{1}-A_{2}\right)\left(-e_{1} g_{2}+B_{1} \widetilde{\mathcal{R}}_{\tau, B_{1}}\left\{e_{1} g_{2}\right\}\right) e^{\tau \Phi}, b_{\tau} e^{\mathcal{B}_{2}-\tau \Phi}+c_{\tau} e^{\mathcal{A}_{2}-\tau \Phi}\right)_{L^{2}(\Omega)} \\
& +2\left(\left(B_{1}-B_{2}\right)\left(-\widetilde{\mathcal{R}}_{\tau, B_{1}}\left\{\frac{\partial\left(e_{1} g_{2}\right)}{\partial \bar{z}}\right\} e^{\tau \Phi}+e^{\mathcal{B}_{1}+\tau(\bar{\Phi}-\Phi)} \mathfrak{G}_{2}(\cdot, \tau)\right), b_{\tau} e^{\mathcal{B}_{2}-\tau \Phi}\right)_{L^{2}(\Omega)} \\
& -2\left(\left(B_{1}-B_{2}\right) \frac{\partial}{\partial \bar{z}} \widetilde{\mathcal{R}}_{\tau, B_{1}}\left\{e_{1} g_{2}\right\} e^{\tau \Phi}, c_{\tau} e^{\mathcal{A}_{2}-\tau \bar{\Phi}}\right)_{L^{2}(\Omega)} .
\end{aligned}
$$

Integrating by parts and using (4.11), we have

$$
\begin{aligned}
-2\left(\left(B_{1}-\right.\right. & \left.\left.B_{2}\right) \frac{\partial}{\partial \bar{z}} \widetilde{\mathcal{R}}_{\tau, B_{1}}\left\{e_{1} g_{2}\right\} e^{\tau \Phi}, c_{\tau} e^{\mathcal{A}_{2}-\tau \bar{\Phi}}\right)_{L^{2}(\Omega)} \\
= & -2\left(\left(B_{1}-B_{2}\right) \frac{\partial}{\partial \bar{z}} \widetilde{\mathcal{R}}_{\tau, B_{1}}\left\{e_{1} g_{2}\right\}, c_{\tau} e^{\mathcal{A}_{2}}\right)_{L^{2}(\Omega)} \\
= & -2 \int_{\Omega} \widetilde{\mathcal{R}}_{\tau, B_{1}}\left\{e_{1} g_{2}\right\} \frac{\partial}{\partial \bar{z}}\left(\left(B_{1}-B_{2}\right) \overline{c_{\tau}} e^{\overline{\mathcal{A}_{2}}}\right) d x \\
& -\int_{\partial \Omega}\left(B_{1}-B_{2}\right)\left(\nu_{1}+i \nu_{2}\right) \widetilde{\mathcal{R}}_{\tau, B_{1}}\left\{e_{1} g_{2}\right\} \overline{c_{\tau}} e^{\overline{\mathcal{A}_{2}}} d \sigma \\
= & \int_{\Omega} \frac{e_{1} g_{2}}{\tau \partial_{z} \Phi} \frac{\partial}{\partial \bar{z}}\left(\left(B_{1}-B_{2}\right) \bar{c} e^{\overline{\mathcal{A}_{2}}}\right) d x+o(1 / \tau) \quad \text { as }|\tau| \rightarrow+\infty
\end{aligned}
$$

The stationary phase argument implies the formula

$$
\begin{aligned}
-2( & \left.\left(B_{1}-B_{2}\right) \widetilde{\mathcal{R}}_{\tau, B_{1}}\left\{\frac{\partial\left(e_{1} g_{2}\right)}{\partial \bar{z}}\right\} e^{\tau \Phi}, b_{\tau} e^{\mathcal{B}_{2}-\tau \Phi}\right)_{L^{2}(\Omega)} \\
& =-\int_{\Omega}\left(B_{1}-B_{2}\right) \partial_{z}^{-1}\left\{\frac{\partial\left(e_{1} g_{2}\right)}{\partial \bar{z}} e^{-\mathcal{B}_{1}+\tau(\Phi-\bar{\Phi})}\right\} \overline{b_{\tau}} e^{\mathcal{B}_{1}+\overline{\mathcal{B}_{2}}} d x \\
& =\frac{1}{\pi} \int_{\Omega} \frac{\partial\left(e_{1} g_{2}\right)}{\partial \bar{\zeta}} e^{-\mathcal{B}_{1}+\tau(\Phi-\bar{\Phi})}\left(\int_{\Omega} \frac{\left(B_{1}-B_{2}\right) \overline{b_{\tau}} e^{\mathcal{B}_{1}+\overline{\mathcal{B}_{2}}}}{\bar{\zeta}-\bar{z}} d x\right) d \xi_{1} d \xi_{2} \\
& =\frac{\Lambda_{+}(\tau, \widetilde{x})}{\tau} \frac{\partial g_{2}}{\partial \bar{z}}(\widetilde{x}) e^{-\mathcal{B}_{1}(\widetilde{x})} \int_{\Omega} \frac{\left(B_{1}-B_{2}\right) \overline{b_{\tau}} e^{\mathcal{B}_{1}-\overline{\mathcal{B}_{2}}}}{\overline{\widetilde{z}}-\bar{z}} d x+o(1 / \tau) .
\end{aligned}
$$

By (8.11) we have the asymptotic formula

$$
\begin{aligned}
& 2\left(\left(B_{1}-B_{2}\right)\left(e^{\mathcal{B}_{1}} e^{\tau \bar{\Phi}} \mathfrak{G}_{2}, b_{\tau} e^{\mathcal{B}_{2}-\tau \Phi}\right)_{L^{2}(\Omega)}\right. \\
= & 2\left(\left(B_{1}-B_{2}\right) e^{\mathcal{B}_{1}} \mathfrak{G}_{2}, b_{\tau} e^{\mathcal{B}_{2}}\right)_{L^{2}(\Omega)}=2 \int_{\Omega}\left(B_{1}-B_{2}\right) e^{\mathcal{B}_{1}+\overline{\mathcal{B}_{2}}} \overline{b_{\tau}(z)} \mathfrak{G}_{2}(x, \tau) d x \\
= & -4 \int_{\Omega} \frac{\partial}{\partial z} e^{\mathcal{B}_{1}+\overline{\mathcal{B}_{2}}} \overline{b_{\tau}(z)} \mathfrak{G}_{2}(x, \tau) d x \\
= & -2 \int_{\partial \Omega}\left(\nu_{1}-i \nu_{2}\right) e^{\mathcal{B}_{1}+\overline{\mathcal{B}_{2}}} \overline{b_{\tau}(z)} \mathfrak{G}_{2}(x, \tau) d \sigma+4 \int_{\Omega} e^{\mathcal{B}_{1}+\overline{\mathcal{B}_{2}}} \overline{b_{\tau}(z)} \frac{\partial}{\partial z} \mathfrak{G}_{2}(x, \tau) d x
\end{aligned}
$$


Observe that

$$
\begin{aligned}
\frac{\partial}{\partial z} \mathfrak{G}_{2}(x, \tau) & =-\frac{1}{4 \pi} \frac{\partial B_{1}}{\partial \bar{z}} \int_{\Omega} \frac{\left(e_{1} g_{2} e^{-\mathcal{B}_{1}}\right)\left(\xi_{1}, \xi_{2}\right)}{\bar{\zeta}-\bar{z}} e^{\tau(\Phi(\zeta)-\overline{\Phi(\zeta)})} d \xi_{1} d \xi_{2} \\
& =\frac{e^{-\mathcal{B}_{1}}}{2} e^{\tau(\Phi-\bar{\Phi})} \widetilde{\mathcal{R}}_{\tau, B_{1}}\left\{e_{1} g_{2}\right\}
\end{aligned}
$$

Then by Proposition 3.4 we have

$$
\begin{aligned}
& 4 \int_{\Omega} e^{\mathcal{B}_{1}+\overline{\mathcal{B}_{2}}} \overline{b_{\tau}(z)} \frac{\partial}{\partial z} \mathfrak{G}_{2}(x, \tau) d x \\
& \quad=2 \int_{\Omega} e^{\mathcal{B}_{1}+\overline{\mathcal{B}_{2}}} \overline{b_{\tau}(z)} e^{-\mathcal{B}_{1}} e^{\tau(\Phi-\bar{\Phi})} \widetilde{\mathcal{R}}_{\tau, B_{1}}\left\{e_{1} g_{2}\right\} d x \\
& =\int_{\Omega} e^{\mathcal{B}_{1}+\overline{\mathcal{B}_{2}}} \overline{b_{\tau}(z)} e^{-\mathcal{B}_{1}} e^{\tau(\Phi-\bar{\Phi})} \frac{e_{1} g_{2}}{\tau \partial_{z} \Phi} d x=o(1 / \tau) \quad \text { as }|\tau| \rightarrow+\infty
\end{aligned}
$$

By (8.21)-(8.22) we have

$$
\begin{aligned}
\mathfrak{L}_{1}= & \left(\left(A_{1}-A_{2}\right)\left(-e_{1} g_{2}+\frac{B_{1} e_{1} g_{2}}{2 \tau \partial_{z} \Phi}\right), c_{\tau} e^{\mathcal{A}_{2}}\right)_{L^{2}(\Omega)} \\
& +\int_{\Omega} \frac{e_{1} g_{2}}{\tau \partial_{z} \Phi} \frac{\partial}{\partial \bar{z}}\left(\left(B_{1}-B_{2}\right) \overline{c_{\tau}} e^{\overline{\mathcal{A}_{2}}}\right) d x \\
& +\frac{\Lambda_{+}(\tau, \widetilde{x})}{\tau} \frac{\partial g_{2}}{\partial \bar{z}}(\widetilde{x}) e^{-\mathcal{B}_{1}(\widetilde{x})} \int_{\Omega} \frac{\left(B_{1}-B_{2}\right) \bar{b} e^{\mathcal{B}_{1}+\overline{\mathcal{B}_{2}}}}{\overline{\widetilde{z}}-\bar{z}} d x \\
& -2 \int_{\partial \Omega}\left(\nu_{1}-i \nu_{2}\right) e^{\mathcal{B}_{1}+\overline{\mathcal{B}_{2}}} \frac{b_{\tau}(z)}{\mathfrak{G}_{2}}(x, \tau) d \sigma+o(1 / \tau) \quad \text { as }|\tau| \rightarrow+\infty .
\end{aligned}
$$

Recall that $V_{1}=-e^{-\tau \Phi} \widetilde{\mathcal{R}}_{-\tau,-} \overline{A_{2}}\left\{e_{1} g_{3}\right\}$ and $V_{2}=-e^{-\tau \bar{\Phi}} \mathcal{R}_{\tau,-\overline{B_{2}}}\left\{e_{1} g_{4}\right\}$.

By Proposition 3.2 we conclude that

$$
\begin{aligned}
& 2 \frac{\partial V_{1}}{\partial z}=\left(-e_{1} g_{3}+\overline{A_{2}} \widetilde{\mathcal{R}}_{-\tau, \overline{A_{2}}}\left\{e_{1} g_{3}\right\}\right) e^{-\tau \Phi}, \\
& 2 \frac{\partial V_{2}}{\partial \bar{z}}=\left(-e_{1} g_{4}+\overline{B_{2}} \mathcal{R}_{\tau,-\bar{B}_{2}}\left\{e_{1} g_{4}\right\}\right) e^{-\tau \bar{\Phi}} .
\end{aligned}
$$

Similarly to (5.15) and (5.17) we calculate

$$
\begin{aligned}
& \frac{\partial V_{1}}{\partial \bar{z}}=-e^{-\tau \Phi} \widetilde{\mathcal{R}}_{-\tau,-\overline{A_{2}}}\left\{\frac{\partial\left(e_{1} g_{3}\right)}{\partial \bar{z}}\right\}+e^{-\tau \bar{\Phi}+\mathcal{A}_{2}} \mathfrak{G}_{3}(\cdot, \tau), \\
& \frac{\partial V_{2}}{\partial z}=-e^{-\tau \bar{\Phi}} \mathcal{R}_{\tau,-\overline{B_{2}}}\left\{\frac{\partial\left(e_{1} g_{4}\right)}{\partial z}\right\}+e^{-\tau \Phi+\mathcal{B}_{2}} \mathfrak{G}_{4}(\cdot, \tau) .
\end{aligned}
$$

Using (3.2) and integrating by parts, we obtain

$$
\mathfrak{L}_{2} \equiv\left(2\left(A_{1}-A_{2}\right) \frac{\partial}{\partial z}\left(a_{\tau} e^{\mathcal{A}_{1}+\tau \Phi}+d_{\tau} e^{\mathcal{B}_{1}+\tau \bar{\Phi}}\right), V_{1}+V_{2}\right)_{L^{2}(\Omega)}
$$


1050

$$
\begin{aligned}
= & -\left(\left(A_{1}-A_{2}\right) d_{\tau} B_{1} e^{\mathcal{B}_{1}} e^{\tau \bar{\Phi}}, V_{1}+V_{2}\right)_{L^{2}(\Omega)} \\
& +\left(\left(A_{1}-A_{2}\right)\left(\nu_{1}-i \nu_{2}\right) a_{\tau} e^{\mathcal{A}_{1}+\tau \Phi}, V_{1}+V_{2}\right)_{L^{2}(\partial \Omega)} \\
& -\left(2 \frac{\partial}{\partial z}\left(A_{1}-A_{2}\right) a_{\tau} e^{\mathcal{A}_{1}+\tau \Phi}, V_{1}+V_{2}\right)_{L^{2}(\Omega)} \\
& -\left(\left(A_{1}-A_{2}\right) a_{\tau} e^{\mathcal{A}_{1}+\tau \Phi}, 2\left(\frac{\partial V_{1}}{\partial \bar{z}}+\frac{\partial V_{2}}{\partial \bar{z}}\right)\right)_{L^{2}(\Omega)} .
\end{aligned}
$$

We observe that by (8.26), (8.11), Propositions 3.4 and 3.3 and the equality $\frac{\partial \mathfrak{G}_{3}}{\partial z}=-\frac{1}{2} e^{-\mathcal{A}_{2}} \frac{\partial \overline{A_{2}}}{\partial \bar{z}} e^{\tau(\bar{\Phi}-\Phi)} \widetilde{\mathcal{R}}_{-\tau,-\overline{A_{2}}}\left(e_{1} g_{3}\right)$, we have

$$
\begin{aligned}
( & \left.\left(A_{1}-A_{2}\right) a_{\tau} e^{\mathcal{A}_{1}+\tau \Phi}, 2 \frac{\partial V_{1}}{\partial \bar{z}}\right)_{L^{2}(\Omega)}=-4 \int_{\Omega} a_{\tau}(z) \frac{\partial}{\partial \bar{z}} e^{\mathcal{A}_{1}+\overline{\mathcal{A}_{2}}} \overline{\mathfrak{G}_{3}(x, \tau)} d x \\
& +\left(\left(A_{1}-A_{2}\right) a_{\tau} e^{\mathcal{A}_{1}+\tau \Phi},-e^{-\tau \Phi} \widetilde{\mathcal{R}_{-\tau,-}} \overline{A_{2}}\left\{\frac{\partial\left(e_{1} g_{3}\right)}{\partial \bar{z}}\right\}\right)_{L^{2}(\Omega)}+o(1 / \tau) \\
= & -2 \int_{\partial \Omega} a_{\tau}(z) \overline{\mathfrak{G}_{3}(x, \tau)}\left(\nu_{1}+i \nu_{2}\right) e^{\mathcal{A}_{1}+\overline{\mathcal{A}_{2}}} d \sigma \\
& +\frac{1}{\pi} \int_{\Omega} \frac{\overline{\partial\left(e_{1} g_{3}\right)}}{\partial \bar{\zeta}} e^{\tau(\Phi-\bar{\Phi})-\overline{\mathcal{A}_{2}}}\left(\int_{\Omega} \frac{\left(A_{1}-A_{2}\right) a_{\tau} e^{\mathcal{A}_{1}+\overline{\mathcal{A}_{2}}}}{\zeta-z} d x\right) d \xi_{1} d \xi_{2}+o(1 / \tau) \\
= & \frac{\Lambda_{+}(\tau, \widetilde{x})}{\tau} \frac{\overline{\partial g_{3}}}{\partial \bar{z}}(\widetilde{x}) e^{-\overline{\mathcal{A}_{2}}(\widetilde{x})} \int_{\Omega} \frac{\left(A_{1}-A_{2}\right) a e^{\mathcal{A}_{1}+\overline{\mathcal{A}_{2}}}}{\widetilde{z}-z} d x \quad \text { as }|\tau| \rightarrow+\infty \\
& -2 \int_{\partial \Omega} a_{\tau}(z) \overline{\mathfrak{G}_{3}(x, \tau)}\left(\nu_{1}+i \nu_{2}\right) e^{\mathcal{A}_{1}+\overline{\mathcal{A}_{2}}} d \sigma+o(1 / \tau)
\end{aligned}
$$

Hence, using (8.25) we have

$$
\begin{aligned}
\mathfrak{L}_{2}=( & \left.\left(A_{1}-A_{2}\right) d_{\tau} B_{1} e^{\mathcal{B}_{1}}, \widetilde{\mathcal{R}}_{-\tau,-\overline{A_{2}}}\left\{e_{1} g_{3}\right\}\right)_{L^{2}(\Omega)} \\
& +\left(\left(A_{1}-A_{2}\right)\left(\nu_{1}-i \nu_{2}\right) a_{\tau} e^{\mathcal{A}_{1}+\tau \Phi}, V_{1}+V_{2}\right)_{L^{2}(\partial \Omega)} \\
& +\left(2 \frac{\partial}{\partial z}\left(A_{1}-A_{2}\right) a_{\tau} e^{\mathcal{A}_{1}}, \mathcal{R}_{\tau,-\overline{B_{2}}}\left\{e_{1} g_{4}\right\}\right)_{L^{2}(\Omega)} \\
& -\frac{\Lambda_{+}(\tau, \widetilde{x})}{\tau} \frac{\overline{\partial g_{3}}}{\partial \bar{z}}(\widetilde{x}) e^{-\overline{\mathcal{A}_{2}}(\widetilde{x})} \int_{\Omega} \frac{\left(A_{1}-A_{2}\right) a e^{\mathcal{A}_{1}+\overline{\mathcal{A}_{2}}}}{\widetilde{z}-z} d x \\
& +\left(\left(A_{1}-A_{2}\right) a_{\tau} e^{\mathcal{A}_{1}},-e_{1} g_{4}-\overline{B_{2}} \mathcal{R}_{\tau,-\overline{B_{2}}}\left\{e_{1} g_{4}\right\}\right)_{L^{2}(\Omega)}+o(1 / \tau) \\
& +2 \int_{\partial \Omega} a_{\tau}(z) \overline{\mathfrak{G}_{3}(x, \tau)}\left(\nu_{1}+i \nu_{2}\right) e^{\mathcal{A}_{1}+\overline{\mathcal{A}_{2}}} d \sigma \quad \text { as }|\tau| \rightarrow+\infty .
\end{aligned}
$$

By (4.40) and (4.39) we obtain

$$
\left(\left(A_{1}-A_{2}\right)\left(\nu_{1}-i \nu_{2}\right) a_{\tau} e^{\mathcal{A}_{1}+\tau \Phi}, V_{1}+V_{2}\right)_{L^{2}(\partial \Omega)}=o(1 / \tau) \quad \text { as }|\tau| \rightarrow+\infty .
$$


Therefore, by Proposition 3.4, we have

$$
\begin{aligned}
\mathfrak{L}_{2}= & -\left(\left(A_{1}-A_{2}\right) d_{\tau} B_{1} e^{\mathcal{B}_{1}}, \frac{e_{1} g_{3}}{2 \tau \partial_{z} \Phi}\right)_{L^{2}(\Omega)} \\
& -\left(\frac{\partial}{\partial z}\left(A_{1}-A_{2}\right) a_{\tau} e^{\mathcal{A}_{1}}, \frac{e_{1} g_{4}}{\tau \overline{\partial_{z} \Phi}}\right)_{L^{2}(\Omega)} \\
& +\left(\left(A_{1}-A_{2}\right) a_{\tau} e^{\mathcal{A}_{1}},-e_{1} g_{4}+\overline{B_{2}} \frac{e_{1} g_{4}}{2 \tau \overline{\partial_{z} \Phi}}\right)_{L^{2}(\Omega)} \\
& -\frac{\Lambda_{+}(\tau, \widetilde{x})}{\tau} \frac{\overline{\partial g_{3}}}{\partial \bar{z}}(\widetilde{x}) e^{-\overline{\mathcal{A}_{2}}(\widetilde{x})} \int_{\Omega} \frac{\left(A_{1}-A_{2}\right) a e^{\mathcal{A}_{1}+\overline{\mathcal{A}_{2}}}}{\widetilde{z}-z} d x \\
& +2 \int_{\partial \Omega} a_{\tau}(z) \overline{\mathfrak{G}_{3}(x, \tau)}\left(\nu_{1}+i \nu_{2}\right) e^{\mathcal{A}_{1}+\overline{\mathcal{A}_{2}}} d \sigma+o(1 / \tau) \quad \text { as }|\tau| \rightarrow+\infty
\end{aligned}
$$

Integrating by parts, we compute

$$
\begin{aligned}
\mathfrak{L}_{3} \equiv & \left(2\left(B_{1}-B_{2}\right) \frac{\partial}{\partial \bar{z}}\left(a_{\tau} e^{\mathcal{A}_{1}+\tau \Phi}+d_{\tau} e^{\mathcal{B}_{1}+\tau \bar{\Phi}}\right), V_{1}+V_{2}\right)_{L^{2}(\Omega)} \\
= & -\left(2 \frac{\partial}{\partial \bar{z}}\left(B_{1}-B_{2}\right) d_{\tau} e^{\mathcal{B}_{1}+\tau \bar{\Phi}}, V_{1}+V_{2}\right)_{L^{2}(\Omega)} \\
& -\left(\left(B_{1}-B_{2}\right) A_{1} a_{\tau} e^{\mathcal{A}_{1}+\tau \Phi}, V_{1}+V_{2}\right)_{L^{2}(\Omega)} \\
& +\left(\left(\nu_{1}+i \nu_{2}\right)\left(B_{1}-B_{2}\right) d_{\tau} e^{\mathcal{B}_{1}+\tau \Phi}, V_{1}+V_{2}\right)_{L^{2}(\partial \Omega)} \\
& -\left(\left(B_{1}-B_{2}\right) d_{\tau} e^{\mathcal{B}_{1}+\tau \bar{\Phi}}, 2\left(\frac{\partial V_{1}}{\partial z}+\frac{\partial V_{2}}{\partial z}\right)\right)_{L^{2}(\Omega)} .
\end{aligned}
$$

We observe that by $(8.11),(8.27)$, Propositions 3.4 and 3.3 and the equality $\frac{\partial \mathfrak{G}_{4}}{\partial \bar{z}}=-\frac{1}{2} e^{-\mathcal{B}_{2}} \frac{\partial \overline{B_{2}}}{\partial z} e^{\tau(\Phi-\bar{\Phi})} \mathcal{R}_{\tau,-\overline{B_{2}}}\left(e_{1} g_{4}\right)$, we have

$$
\begin{aligned}
& 2\left(\left(B_{1}-B_{2}\right) d_{\tau} e^{\mathcal{B}_{1}+\tau \bar{\Phi}}, \frac{\partial V_{2}}{\partial z}\right)_{L^{2}(\Omega)}=-4 \int_{\Omega} \frac{\partial}{\partial z} e^{\mathcal{B}_{1}+\overline{\mathcal{B}_{2}}} d_{\tau}(\bar{z}) \overline{\mathfrak{G}_{4}(x, \tau)} d x \\
& +2\left(\left(B_{1}-B_{2}\right) d_{\tau} e^{\mathcal{B}_{1}+\tau \bar{\Phi}},-e^{-\tau \bar{\Phi}} \mathcal{R}_{\tau,-\overline{B_{2}}}\left\{\frac{\partial\left(e_{1} g_{4}\right)}{\partial z}\right\}\right)_{L^{2}(\Omega)}+o(1 / \tau) \\
& =-2 \int_{\partial \Omega}\left(\nu_{1}-i \nu_{2}\right) e^{\mathcal{B}_{1}+\overline{\mathcal{B}_{2}}} d_{\tau}(\bar{z}) \overline{\mathfrak{G}_{4}(x, \tau)} d \sigma \\
& +\int_{\Omega}\left(\int_{\Omega} \frac{1}{\pi} \frac{\left(B_{1}-B_{2}\right) d_{\tau} e^{\mathcal{B}_{1}+\overline{\mathcal{B}_{2}}}}{\bar{\zeta}-\bar{z}} d x\right) \overline{\left\{\frac{\partial\left(e_{1} g_{4}\right)}{\partial z}\right\}} e^{-\overline{\mathcal{B}_{2}}+\tau(\bar{\Phi}-\Phi)} d \xi_{1} d \xi_{2}+o(1 / \tau) \\
& =\frac{\Lambda_{-}(\tau, \widetilde{x})}{\tau} \int_{\Omega} \frac{\left(B_{1}-B_{2}\right) d_{\tau} e^{\mathcal{B}_{1}+\overline{\mathcal{B}_{2}}}}{\overline{\widetilde{z}}-\bar{z}} d x \frac{\overline{\partial g_{4}}(\widetilde{x})}{\partial \zeta} e^{-\overline{\mathcal{B}_{2}}(\widetilde{x})} \\
& -2 \int_{\partial \Omega}\left(\nu_{1}-i \nu_{2}\right) e^{\mathcal{B}_{1}+\overline{B_{2}}} d_{\tau}(\bar{z}) \overline{\mathfrak{G}_{4}(x, \tau)} d \sigma+o(1 / \tau) \quad \text { as }|\tau| \rightarrow+\infty .
\end{aligned}
$$


Hence

$$
\begin{aligned}
\mathfrak{L}_{3}= & \left(2 \frac{\partial}{\partial \bar{z}}\left(B_{1}-B_{2}\right) d_{\tau} e^{\mathcal{B}_{1}}, \widetilde{\mathcal{R}}_{-\tau,-\overline{A_{2}}}\left\{e_{1} g_{3}\right\}\right)_{L^{2}(\Omega)} \\
& -\left(\left(B_{1}-B_{2}\right) A_{1} a_{\tau} e^{\mathcal{A}_{1}}, \mathcal{R}_{\tau,-\overline{B_{2}}}\left\{e_{1} g_{4}\right\}\right)_{L^{2}(\Omega)} \\
& +\left(\left(B_{1}-B_{2}\right) d_{\tau} e^{\mathcal{B}_{1}},-e_{1} g_{3}+\overline{A_{2}} \widetilde{\mathcal{R}}-\tau,-\overline{A_{2}}\left\{e_{1} g_{3}\right\}\right)_{L^{2}(\Omega)} \\
& +\left(\left(\nu_{1}+i \nu_{2}\right)\left(B_{1}-B_{2}\right) d_{\tau} e^{\mathcal{B}_{1}+\tau \Phi}, V_{1}+V_{2}\right)_{L^{2}(\partial \Omega)} \\
& -\frac{\Lambda_{-}(\tau, \widetilde{x})}{\tau} \frac{\overline{\partial g_{4}}}{\partial z}(\widetilde{x}) e^{-\overline{\mathcal{B}_{2}}(\widetilde{x})} \int_{\Omega} \frac{\left(B_{1}-B_{2}\right) d e^{\mathcal{B}_{1}+\overline{\mathcal{B}_{2}}}}{\overline{\widetilde{z}}-\bar{z}} d x \\
& +2 \int_{\partial \Omega}\left(\nu_{1}-i \nu_{2}\right) e^{\mathcal{B}_{1}+\overline{\mathcal{B}_{2}}} d_{\tau}(\bar{z}) \overline{\mathfrak{G}_{4}(x, \tau)} d \sigma+o(1 / \tau) \quad \text { as }|\tau| \rightarrow+\infty
\end{aligned}
$$

By (4.40), (4.39) and the stationary phase argument, we obtain

$$
\left(\left(\nu_{1}+i \nu_{2}\right)\left(B_{1}-B_{2}\right) d_{\tau} e^{\mathcal{B}_{1}+\tau \Phi}, V_{1}+V_{2}\right)_{L^{2}(\partial \Omega)}=o(1 / \tau) \quad \text { as }|\tau| \rightarrow+\infty
$$

Therefore, applying Proposition 3.4, we finally conclude that

$$
\begin{aligned}
\mathfrak{L}_{3}= & -2\left(\frac{\partial}{\partial \bar{z}}\left(B_{1}-B_{2}\right) d_{\tau} e^{\mathcal{B}_{1}}, \frac{e_{1} g_{3}}{2 \tau \partial_{z} \Phi}\right)_{L^{2}(\Omega)} \\
& -\left(\left(B_{1}-B_{2}\right) A_{1} a_{\tau} e^{\mathcal{A}_{1}}, \frac{e_{1} g_{4}}{2 \tau \overline{\partial_{z} \Phi}}\right)_{L^{2}(\Omega)} \\
& +\left(\left(B_{1}-B_{2}\right) d_{\tau} e^{\mathcal{B}_{1}},-e_{1} g_{3}-\frac{\overline{A_{2}} e_{1} g_{3}}{2 \tau \partial_{z} \Phi}\right)_{L^{2}(\Omega)} \\
& -\frac{\Lambda_{-}(\tau, \widetilde{x})}{\tau} \frac{\overline{\partial g_{4}}}{\partial z}(\widetilde{x}) e^{-\overline{\mathcal{B}_{2}}(\widetilde{x})} \int_{\Omega} \frac{\left(B_{1}-B_{2}\right) d e^{\mathcal{B}_{1}+\overline{\mathcal{B}_{2}}}}{\overline{\widetilde{z}}-\bar{z}} d x \\
& +2 \int_{\partial \Omega}\left(\nu_{1}-i \nu_{2}\right) e^{\mathcal{B}_{1}+\overline{\mathcal{B}_{2}}} d_{\tau}(\bar{z}) \overline{\mathfrak{G}_{4}(x, \tau)} d \sigma+o(1 / \tau) \quad \text { as }|\tau| \rightarrow+\infty
\end{aligned}
$$

The sum $\sum_{k=0}^{3} \mathfrak{L}_{k}$ is equal to the left-hand side of (5.19). Observe that

$$
\begin{aligned}
& -\frac{\Lambda_{-}(\tau, \widetilde{x})}{\tau} \frac{\overline{\partial g_{4}}}{\partial z}(\widetilde{x}) e^{-\overline{\mathcal{B}_{2}}(\widetilde{x})} \int_{\Omega} \frac{\left(B_{1}-B_{2}\right) d e^{\mathcal{B}_{1}+\overline{\mathcal{B}_{2}}}}{\overline{\widetilde{z}}-\bar{z}} d x \\
& -\frac{\Lambda_{+}(\tau, \widetilde{x})}{\tau} \frac{\overline{\partial g_{3}}}{\partial \bar{z}}(\widetilde{x}) e^{-\overline{\mathcal{A}_{2}}(\widetilde{x})} \int_{\Omega} \frac{\left(A_{1}-A_{2}\right) a e^{\mathcal{A}_{1}+\overline{\mathcal{A}_{2}}}}{\widetilde{z}-z} d x \\
& +\frac{\Lambda_{+}(\tau, \widetilde{x})}{\tau} \frac{\partial g_{2}}{\partial \bar{z}}(\widetilde{x}) e^{-\mathcal{B}_{1}(\widetilde{x})} \int_{\Omega} \frac{\left(B_{1}-B_{2}\right) \bar{b} e^{\mathcal{B}_{1}+\overline{\mathcal{B}_{2}}}}{\overline{\widetilde{z}}-\bar{z}} d x \\
& +\frac{\Lambda_{-}(\tau, \widetilde{x})}{\tau} \frac{\partial g_{1}}{\partial z}(\widetilde{x}) e^{-\mathcal{A}_{1}(\widetilde{x})} \int_{\Omega} \frac{\left(A_{1}-A_{2}\right) \bar{c} e^{\mathcal{A}_{1}+\overline{\mathcal{A}_{2}}}}{\widetilde{z}-z} d x
\end{aligned}
$$




$$
\begin{aligned}
= & \frac{\Lambda_{-}(\tau, \widetilde{x})}{\tau} \frac{\overline{\partial g_{4}}}{\partial z}(\widetilde{x}) e^{-\overline{\mathcal{B}_{2}}(\widetilde{x})} \int_{\partial \Omega} \frac{\left(\nu_{1}-i \nu_{2}\right) d e^{\mathcal{B}_{1}+\overline{\mathcal{B}_{2}}}}{\overline{\widetilde{z}-\bar{z}}} d \sigma \\
& -\frac{\Lambda_{-}(\tau, \widetilde{x})}{\tau} \frac{\partial g_{1}}{\partial z}(\widetilde{x}) e^{-\mathcal{A}_{1}(\widetilde{x})} \int_{\partial \Omega} \frac{\left(\nu_{1}+i \nu_{2}\right) \bar{c} e^{\mathcal{A}_{1}+\overline{\mathcal{A}_{2}}}}{\widetilde{z}-z} d \sigma \\
& +\frac{\Lambda_{+}(\tau, \widetilde{x})}{\tau} \frac{\overline{\partial g_{3}}}{\partial \bar{z}}(\widetilde{x}) e^{-\overline{\mathcal{A}_{2}}(\widetilde{x})} \int_{\partial \Omega} \frac{\left(\nu_{1}+i \nu_{2}\right) a e^{\mathcal{A}_{1}+\overline{\mathcal{A}_{2}}}}{\widetilde{z}-z} d \sigma \\
& -\frac{\Lambda_{+}(\tau, \widetilde{x})}{\tau} \frac{\partial g_{2}}{\partial \bar{z}}(\widetilde{x}) e^{-\mathcal{B}_{1}(\widetilde{x})} \int_{\partial \Omega} \frac{\left(\nu_{1}-i \nu_{2}\right) \bar{b} e^{\mathcal{B}_{1}+\overline{\mathcal{B}_{2}}}}{\widetilde{\widetilde{z}}-\bar{z}} d \sigma \\
& -\frac{2 \pi}{\tau}\left(\mathcal{Q}_{+} a \bar{b} e^{\mathcal{A}_{1}+\overline{\mathcal{B}_{2}}} \Lambda_{+}(\tau, \widetilde{x})+\mathcal{Q}_{-} \bar{c} d e^{\mathcal{B}_{1}+\overline{\mathcal{A}_{2}}} \Lambda_{-}(\tau, \widetilde{x})\right)(\widetilde{x}) .
\end{aligned}
$$

By $(8.17),(8.23),(8.29),(8.31)$ and $(8.32)$, there exist numbers $\kappa, \kappa_{0}$ such that the asymptotic formula (5.19) holds true.

\section{Acknowledgments}

The first named author was partly supported by NSF grant DMS 0808130, and the second named author was partly supported by NSF, a Walker Family Endowed Professorship, a Chancellor Professorship at UC Berkeley and a Senior Clay Award. The visits of O. Imanuvilov to Seattle were supported by NSF and the Department of Mathematics of the University of Washington. O. Imanuvilov also thanks the Global COE Program "The Research and Training Center for New Development in Mathematics" for support of visits to the University of Tokyo. The authors thank Professor J. Sawon for discussions on the proof of Corollary 1.1. The authors sincerely thank the anonymous referee for many valuable comments.

\section{References}

[1] L. Ahlfors, Lectures on quasiconformal mappings, Van Nostrand, Princeton, NJ, 1966. Zbl 0138.06002 MR 0200442

[2] K. Astala and L. Päivärinta, Calderón's inverse conductivity problem in the plane, Ann. of Math. 163 (2006), 265-299. Zbl 1111.35004 MR 2195135

[3] K. Astala, L. Päivärinta and M. Lassas, Calderón's inverse problem for anisotropic conductivity in the plane, Comm. Partial Differential Equations 30 (2005), 207-224. Zbl 1129.35483 MR 2131051

[4] N. Bleistein and R. Handelsman, Asymptotic expansions of integrals, Dover Publ., New York, 1986. MR 0863284

[5] A. Böttcher and B. Silbermann, Analysis of Toeplitz operators, Springer, Berlin, 2006. Zbl 1098.47002 MR 2223704

[6] R. Brown and G. Uhlmann, Uniqueness in the inverse conductivity problem with less regular conductivities in two dimensions, Comm. Partial Differential Equations 22 (1997), 10091027. Zbl 0884.35167 MR 1452176

[7] A. Bukhgeim, Recovering the potential from Cauchy data in two dimensions, J. Inverse Ill-Posed Problems 16 (2008), 19-34. Zbl 1142.30018 MR 2387648 
[8] A. Bukhgeim and G. Uhlmann, Recovering a potential from partial Cauchy data, Comm. Partial Differential Equations 27 (2002), 653-668. Zbl 0998.35063 MR 1900557

[9] A. P. Calderón, On an inverse boundary value problem, in Seminar on numerical analysis and its applications to continuum physics, Soc. Brasil. Mat., Río de Janeiro, 1980, 65-73. MR 0590275

[10] J. Cheng, G. Nakamura and E. Somersalo, Uniqueness of identifying the convection term, in Second Japan-Korea joint seminar on inverse problems and related topics (Seoul, 2001), Comm. Korean Math. Soc. 16 (2001), 405-413. Zbl 1101.35398 MR 1843524

[11] J. Cheng and M. Yamamoto, Determination of two convection coefficients from Dirichlet to Neumann map in the two-dimensional case, SIAM J. Math. Anal. 35 (2004), 1371-1393. Zbl 1061.35164 MR 2083783

[12] D. Dos Santos Ferreira, C. Kenig, J. Sjöstrand and G. Uhlmann, Determining a magnetic Schrödinger operator from partial Cauchy data, Comm. Math. Phys. 271 (2007), 467-488. Zbl 1148.35096 MR 2287913

[13] C. Guillarmou and L. Tzou, Calderón inverse problem with partial data on Riemann surfaces, Duke Math. J. 158 (2011), 83-120. Zbl 1222.35212 MR 2794369

[14] L. Hörmander, Linear partial differential operators, Springer, Berlin, 1963. Zbl 0108.09301 MR 0161012

[15] O. Imanuvilov, G. Uhlmann and M. Yamamoto, The Calderón problem with partial data in two dimensions, J. Amer. Math. Soc. 23 (2010), 655-691. Zbl 1201.35183 MR 2629983

[16] H. Kang and Y. Kihyun, Boundary determination of conductivities and Riemannian metrics via local Dirichlet-to-Neumann operator, SIAM J. Math. Anal. 34 (2003), 719-735. Zbl 1044.35106 MR 1970890

[17] H. Kang and G. Uhlmann, Inverse problems for the Pauli Hamiltonian in two dimensions, J. Fourier Anal. Appl. 10 (2004), 201-215. Zbl 1081.35141 MR 2054308

[18] C. Kenig, J. Sjöstrand and G. Uhlmann, The Calderón problem with partial data, Ann. of Math. 165 (2007), 567-591. Zbl 1127.35079 MR 2299741

[19] K. Knudsen and M. Salo, Determining nonsmooth first order terms from partial boundary measurements, Inverse Problems and Imaging 1 (2007), 349-369. Zbl 1122.35152 MR 2282273

[20] J. Lee and G. Uhlmann, Determining anisotropic real-analytic conductivities by boundary measurements, Comm. Pure Appl. Math. 42 (1989), 1097-1112. Zbl 0702.35036 MR 1029119

[21] A. Nachman, Global uniqueness for a two-dimensional inverse boundary value problem, Ann. of Math. 143 (1996), 71-96. Zbl 0857.35135 MR 1370758

[22] G. Nakamura, Z. Sun and G. Uhlmann, Global identifiability for an inverse problem for the Schrödinger equation in a magnetic field, Math. Ann. 303 (1995), 377-388. Zbl 0843.35134 MR 1354996

[23] M. Salo, Inverse problems for nonsmooth first order perturbations of the Laplacian, Ann. Acad. Sci. Fenn. Math. Diss. 139 (2004). Zbl 1059.35175 MR 2105191

[24] Semiclassical pseudodifferential calculus and the reconstruction of a magnetic field, Comm. Partial Differential Equations 31 (2006), 1639-1666. Zbl 1119.35119 MR 2273968

[25] S. Shamir, Regularization of mixed second-order elliptic problems, Israel J. Math. 6 (1968), 150-168. Zbl 0157.18202 MR 0239272

[26] Z. Sun, An inverse boundary value problem for the Schrödinger operator with vector potentials in two dimensions, Comm. Partial Differential Equations 18 (1993), 83-124. Zbl 0781.35073 MR 1211726

[27] Z. Sun and G. Uhlmann, Anisotropic inverse problems in two dimensions, Inverse Problems 19 (2003), 1001-1010. Zbl 1054.35139 MR 2024685 
[28] R. Temam, Navier-Stokes equations, theory and numerical analysis, AMS Chelsea, Providence, 2001. Zbl 0981.35001 MR 1846644

[29] I. Vekua, Generalized analytic functions, Pergamon Press, Oxford, 1962. Zbl 0100.07603 MR 0150320

[30] W. Wendland, Elliptic systems in the plane, Pitman, London, 1979. Z Zbl 0396.35001 MR 0518816 